\title{
Organometallic ruthenium(II)-arene complexes with triphenylphosphine amino acid bioconjugates: Synthesis, characterization and biological properties
}

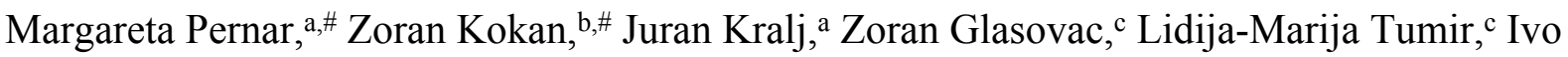
Piantanida, ${ }^{\mathrm{c}}$ Domagoj Eljuga ${ }^{\mathrm{d}}{ }^{\text {Iztok Turel, }},{ }^{\mathrm{e}}$ Anamaria Brozovic, ${ }^{\mathrm{a}, *}$ Srećko I. Kirin, ${ }^{\mathrm{b}, *}$

a Division of Molecular Biology, Ruđer Bošković Institute, Bijenička cesta 54, HR-10000 Zagreb, Croatia

b Division of Materials Chemistry, Ruđer Bošković Institute, Bijenička cesta 54, HR-10000 Zagreb, Croatia

c Division of Organic Chemistry and Biochemistry, Ruđer Bošković Institute, Bijenička cesta 54, HR-10000 Zagreb, Croatia

d Department for Oncoplastic and Reconstructive Surgery, University Hospital for Tumors, University Clinical Hospital Centre Sisters of Mercy, HR-10000 Zagreb, Croatia

e Faculty of Chemistry and Chemical Technology, University of Ljubljana, Večna pot 113, SLO-1000 Ljubljana, Slovenia

\# equal contribution

*corresponding authors

E-mail: Anamaria.Brozovic@irb.hr, Srecko.Kirin@irb.hr

Key words: Ruthenium; $p$-Cymene; Phosphine ligands; Cytotoxicity; Cell death

\section{Highlights.}

A set of ( $p$-cymene)-ruthenium amino acid bioconjugates $\mathbf{1}$ and $\mathbf{2}$ is reported.

The bioconjugates 2 show micromolar affinity for proteins.

Increased potency and selectivity of $\mathbf{2}$ towards cancer cell lines is detected.

The cytotoxicity of bioconjugates $\mathbf{2}$ is in correlation with their BSA binding constants.

Cell stress response includes increase of cells in S phase of cell cycle, induction of autophagy and use of GSH as detoxification mechanism. 


\section{Organometallic ruthenium(II)-arene complexes with triphenylphosphine amino acid bioconjugates: Synthesis, characterization and biological properties}

Margareta Pernar, ${ }^{\text {a, }}$ Zoran Kokan, ${ }^{\text {b, }}$ Juran Kralj, ${ }^{\text {a } Z o r a n ~ G l a s o v a c, ~}{ }^{c}$ Lidija-Marija Tumir, ${ }^{\mathrm{c}}$ Ivo Piantanida, ${ }^{\mathrm{c}}$ Domagoj Eljuga ${ }^{\mathrm{d}}{ }^{\text {Iztok Turel, }},{ }^{\mathrm{e}}$ Anamaria Brozovic, ${ }^{\mathrm{a}, *}$ Srećko I. Kirin, ${ }^{\mathrm{b}, *}$

a Division of Molecular Biology, Ruđer Bošković Institute, Bijenička cesta 54, HR-10000 Zagreb, Croatia

b Division of Materials Chemistry, Ruđer Bošković Institute, Bijenička cesta 54, HR-10000 Zagreb, Croatia

c Division of Organic Chemistry and Biochemistry, Ruđer Bošković Institute, Bijenička cesta 54, HR-10000 Zagreb, Croatia

d Department for Oncoplastic and Reconstructive Surgery, University Hospital for Tumors, University Clinical Hospital Centre Sisters of Mercy, HR-10000 Zagreb, Croatia

e Faculty of Chemistry and Chemical Technology, University of Ljubljana, Večna pot 113, SLO-1000 Ljubljana, Slovenia

\# equal contribution

*corresponding authors

E-mail: Anamaria.Brozovic@irb.hr, Srecko.Kirin@irb.hr

Key words: Ruthenium; $p$-Cymene; Phosphine ligands; Cytotoxicity; Cell death

Graphical abstract.

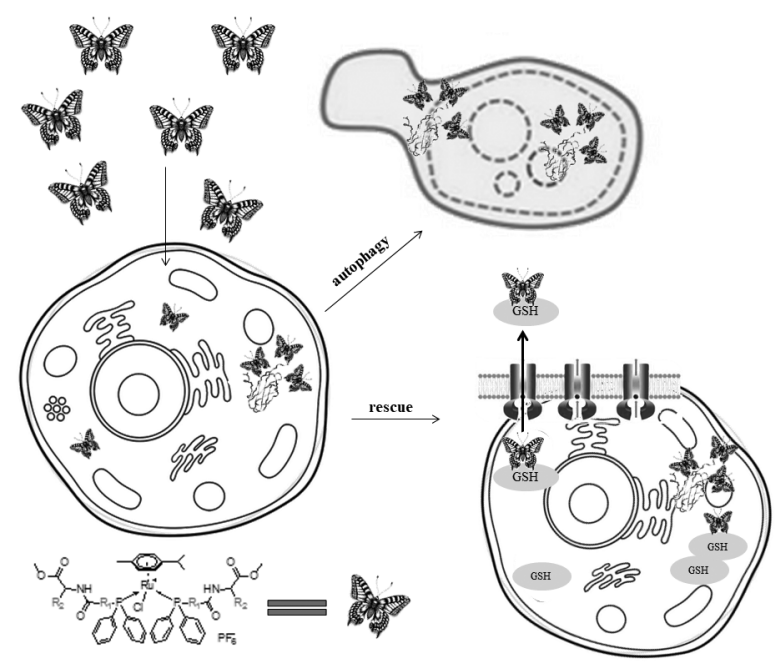




\section{Organometallic ruthenium(II)-arene complexes with triphenylphosphine amino acid bioconjugates: Synthesis, characterization and biological properties}

Margareta Pernar, ${ }^{\text {a, }}$ Zoran Kokan, ${ }^{\text {b, } \# ~ J u r a n ~ K r a l j, ~}{ }^{\text {a } Z o r a n ~ G l a s o v a c, ~}{ }^{\mathrm{c}}$ Lidija-Marija Tumir, ${ }^{\mathrm{c}}$ Ivo Piantanida, ${ }^{\mathrm{c}}$ Domagoj Eljuga, ${ }^{\mathrm{d}}$ Iztok Turel, ${ }^{\mathrm{e}}$ Anamaria Brozovic, ${ }^{\mathrm{a}, *}$ Srećko I. Kirin,,

a Division of Molecular Biology, Ruđer Bošković Institute, Bijenička cesta 54, HR-10000 Zagreb, Croatia

b Division of Materials Chemistry, Ruđer Bošković Institute, Bijenička cesta 54, HR-10000 Zagreb, Croatia

c Division of Organic Chemistry and Biochemistry, Ruđer Bošković Institute, Bijenička cesta 54, HR-10000 Zagreb, Croatia

d Department for Oncoplastic and Reconstructive Surgery, University Hospital for Tumors, University Clinical Hospital Centre Sisters of Mercy, HR-10000 Zagreb, Croatia

e Faculty of Chemistry and Chemical Technology, University of Ljubljana, Večna pot 113, SLO-1000 Ljubljana, Slovenia

\# equal contribution

*corresponding authors

E-mail: Anamaria.Brozovic@irb.hr, Srecko.Kirin@irb.hr

Key words: Ruthenium; $p$-Cymene; Phosphine ligands; Cytotoxicity; Cell death 


\begin{abstract}
.
( $p$-Cymene)-ruthenium bioconjugates $\mathbf{M L}$ (1) and $\mathbf{M L}_{\mathbf{2}}$ (2), bearing phosphane ligands substituted with chiral or non-chiral amino acid esters, $\mathbf{L}$, were synthetized and characterized by instrumental methods (NMR, CD, MS) and DFT calculations (using the wB97xD functional). Cytotoxic activity of complexes $\mathbf{1}$ and $\mathbf{2}$ was investigated by using human cervical carcinoma cell line (HeLa) and MTT assay. Four $\left(\mathbf{2}_{\mathbf{p G}}, \mathbf{2}_{\mathbf{p A}}, \mathbf{2}_{\mathbf{m G}}\right.$ and $\left.\mathbf{2}_{\mathbf{m A}}\right)$ out of ten synthesized ruthenium complexes showed significant toxicity, with $\mathrm{IC}_{50}$ values of 5 to 30 $\mu \mathrm{M}$. Evaluation of the potential biomolecular targets of bioconjugates 2 by UV-Vis, fluorescence and CD spectroscopy revealed no measurable interaction with DNA, but micromolar affinity for proteins. The cytotoxicity of bioconjugates $\mathbf{2}$ is in correlation with their BSA binding constants, i. e. bioconjugates with lower $\mathrm{IC}_{50}$ values show higher binding affinities towards BSA. Compound $\mathbf{2}_{\mathbf{m G}}$ with value of $\mathrm{IC}_{50} 16 \mu \mathrm{M}$ was selected for further biological characterization. The higher level of toxicity towards tumor compared to normal cell lines indicates its selective activity, important characteristic for potential medical use. It was detected $\mathbf{2}_{\mathbf{m G}}$ caused increase of cells in the $\mathrm{S}$ phase of cell cycle and consequential decrease of cells in G0/G1 phase. Additionally, $\mathbf{2}_{\mathbf{m G}}$ caused dose- and time-dependent increase of SubG0/G1 cell population, suggesting its ability to induce programmed cell death. Further investigation determined autophagy as the mode of cell death. The role of GSH in HeLa cells response to investigated organometallic ruthenium complexes was confirmed using specific regulators of GSH synthesis, buthionine sulfoximine and $\mathrm{N}$-acetyl-cysteine. Pre-treatment of cells with ethacrynic acid and probenecid emphasized the role of GSH in detoxification of $\mathbf{2}_{\mathbf{m G}}$ compound. The amount of total ruthenium accumulation in the cell did not correlate with toxicity of $\mathbf{2}_{\mathrm{pG}}, \mathbf{2}_{\mathbf{p A}}, \mathbf{2}_{\mathbf{m G}}$ and $\mathbf{2}_{\mathrm{mA}}$, suggesting structure dependent differences in either cell uptake or kinetics of ruthenium complexes detoxification. We speculate that ruthenium complexes bind protein-based biomolecules further triggering cell death. Based on the gained knowledge, the synthesis and development of more tumor-specific ruthenium-based complexes as potential anticancer drugs can be expected.
\end{abstract}




\section{Introduction.}

Besides the importance of ruthenium $(\mathrm{Ru})$ complexes in catalysis, a major area of research interest is their medical implementation. Although frequently in use, the platinum-based compounds, as an example of prominent inorganic anticancer complexes, have several limitations such as development of drug resistance, side effects and ineffectiveness towards certain tumors (Brozovic and Osmak 2007, Galluzzi et al 2012). In recent years, ruthenium complexes displayed some superior properties over platinum-based drugs (Bergamo and Sava 2015, Vajs et al 2015). Despite the fact that none of the ruthenium complexes is still in clinical use as anticancer drug, prominent representatives like NAMI-A, KP1019, RAPTA and TLD1433 scored success in in vitro and in vivo studies, prompting ruthenium complexes to rapidly become a major area in anticancer drug innovation (Bergamo and Sava 2015, Blunden et al 2014, Domotor et al 2013, Meier-Menches et al 2018, Sersen et al 2015). The wide variety of ruthenium anticancer complexes studied in recent years includes derivatives with amino acids (Rathgeb et al 2014, Scrase et al 2015) and derivatives with triphenylphosphines (Biancalana et al 2017, Chaplin et al 2007, Martinez et al 2017, Millan 2019).

Nowadays, a number of anticancer metal complexes are in the developmental phase and are mostly designed to mimic broadly used cisplatin, known to target DNA. Aside from the DNA damage, cisplatin is able to induce reactive oxygen species (ROS) and endoplasmic reticulum stress, as well as bind to peptides and proteins (Brozovic et al 2010). Similarly, recent studies examining ruthenium anticancer compounds revealed that DNA is not always the primary target and that these compounds bind proteins stronger than to DNA (Gasser et al 2011, Novakova et al 2005, Ravera et al 2004, Scolaro et al 2007). Based on those findings, different modes of ruthenium compounds cytotoxic actions occur, although the exact mechanism is still not known. What is known is the fact that cancer cells are generally growing and multiplying much faster than normal healthy cells, creating a reductive environment due to the raised metabolic rate, higher levels of glutathione $(\mathrm{GSH})$ and a lower $\mathrm{pH}$ (Antonarakis and Emadi 2010). Moreover, it was shown that ruthenium complexes interact with GSH (Wang et al 2005) and GSH-associated enzymes (Lin et al 2013), implying the role of the GSH in cells' defense against toxic damage (Sommer et al 2014).

Amino acid bioconjugates of triphenlyphosphanes are well known ligands for the coordination of transition metals. We have studied the self-assembly and biological properties of their palladium and platinum complexes (Kokan et al 2017, Kokan et al 2018) and successfully used them as monodentate ligands in rhodium catalyzed asymmetric hydrogenations (Kokan and Kirin 2012, Kokan and Kirin 2013, Kokan et al 2014, Opačak et al 2019). A major feature of those metal complexes is the formation of chiral secondary structures responsible for chiral induction in the catalytic cycle. Herein, we explore the synthesis and characterization of ( $p$-cymene)ruthenium complexes $\mathbf{1}$ and $\mathbf{2}$, bearing phosphane ligands $\mathbf{L}$ with chiral or non-chiral amino acid esters. The spacer between the phosphorus and the amino acid was varied in order to investigate hydrogen-bonding propensity of the amino acid substituents. Structural and biological properties of the prepared complexes will be reported.

\section{Results and discussion.}


Synthesis and characterization. The chemical synthesis of the presented organometallic complexes was performed in solution in several steps, Scheme 1. First, the free carboxylic acid of the phosphine precursors $\mathrm{Ph}_{2} \mathrm{P}-p \mathrm{C}_{6} \mathrm{H}_{4}-\mathrm{CO}_{2} \mathrm{H}, \mathrm{Ph}_{2} \mathrm{P}-m \mathrm{C}_{6} \mathrm{H}_{4}-\mathrm{CO}_{2} \mathrm{H}$ or $\mathrm{Ph}_{2} \mathrm{P}_{-} \mathrm{C}_{2} \mathrm{H}_{4}-$ $\mathrm{CO}_{2} \mathrm{H}$ was reacted with $\mathrm{N}$-unprotected amino acid methyl esters of achiral glycine or chiral L-alanine in standard conditions for amide bond formation to yield amino acid triphenylphosphine ligand bioconjugates $\mathbf{L}$, Scheme 1. In the second step, ligand bioconjugates $\mathrm{L}$ and ruthenium precursor $\left[\{(p \mathbf{C y m}) \mathbf{R u C l}\}_{2} \mathbf{C l}_{2}\right]$ were combined to give mono-complexes 1, with 1:1 metal to ligand stoichiometry, general formula $\left[(\boldsymbol{p} \mathbf{C y m}) \mathbf{R u L C l}_{2}\right]$. Finally, in the last synthetic step, mono-complexes react with one additional equivalent of the corresponding ligand and the bis-complexes $\mathbf{2}$ were obtained, [(pCym) RuL $\left.\mathbf{L}_{2} \mathbf{C l}\right]\left(\mathbf{P F}_{\mathbf{6}}\right)$, with 1:2 metal to ligand stoichiometry. In total, six ligands and ten complexes were prepared. Unfortunately, complexes $\mathbf{2}_{\mathrm{aG}}$ and $\mathbf{2}_{\mathrm{aA}}$, although clearly present in the crude reaction mixture, could not be isolated pure enough for further studies.

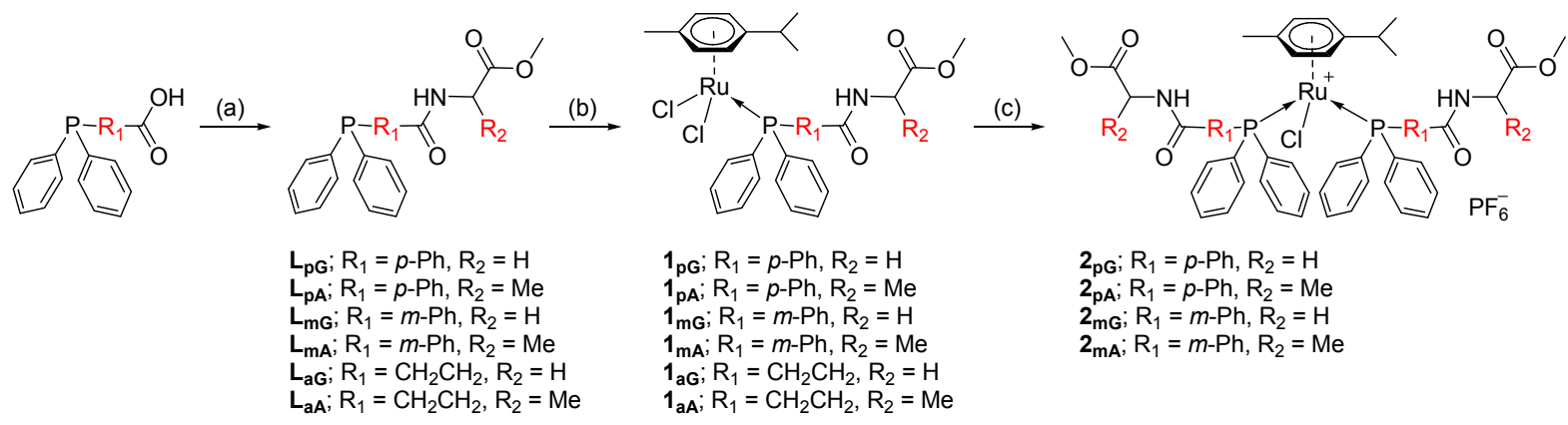

Scheme 1, experimental conditions: (a) TBTU/HOBT/DIPEA/DCM, amino acid ester hydrochloride, RT, 15 h; (b) Di- $\mu$-chlorobis[( $p$-cymene)chlororuthenium(II)]/DCM, RT, 2 h; (c) $1 . \mathrm{NH}_{4} \mathrm{PF}_{6} / \mathrm{AcCN}$, reflux, $45 \mathrm{~min}, 2 . \mathrm{L} / \mathrm{DCM}, \mathrm{RT}, 15 \mathrm{~h}$.

The ligands and complexes were characterized by NMR $\left({ }^{1} \mathrm{H},{ }^{13} \mathrm{C}\right.$ and $\left.{ }^{31} \mathrm{P}\right), \mathrm{CD}$ spectroscopy and MALDI high resolution mass spectrometry. In particular, the ${ }^{31} \mathrm{P}$ shifts of the phosphine moiety in ligands $\mathbf{L}\left(\delta_{\mathrm{P}} \approx-5 \mathrm{ppm}\right)$, mono- and bis-complexes $\mathbf{M L}\left(\delta_{\mathrm{P}} \approx 25 \mathrm{ppm}\right)$ and $\mathbf{M L}_{\mathbf{2}}\left(\delta_{\mathrm{p}}\right.$ $\approx 22 \mathrm{ppm}$ ) clearly indicate binding to the ruthenium metal in the different stoichiometry. The $\mathrm{NH}$ proton chemical shifts for $\mathbf{L}, \mathbf{M L}$ and $\mathbf{M L}_{\mathbf{2}}$ indicate only weak intramolecular hydrogen bonding in $\mathbf{M L}_{2}$ complexes $\left(\delta_{\mathrm{NH}}<7.15 \mathrm{ppm}\right.$ in $\left.\mathrm{CDCl}_{3}\right)$. It is interesting to note that the $\mathrm{C} 2$ proton in $\mathbf{1}_{\mathbf{m G}}$ and $\mathbf{1}_{\mathbf{m A}}$ are unexpectedly highly deshielded $\left(\delta_{\mathrm{H}} \approx 8.9 \mathrm{ppm}\right)$. Selected characteristic NMR data are collected in Table S1.

Computational study. Relative Gibbs energies of the ruthenium complexes were calculated using wB97xD density functional approach, Table S2. This functional was confirmed as very good in rationalizing enantioselectivity in a rhodium catalyzed hydrogenation reaction and in predicting geometries of organic molecules and transition metal complexes including ruthenium complexes (Gridnev and Imamoto 2015, Minenkov et al 2012). Further, this functional is the recommended method when weak interactions like $\pi-\pi$ interactions are expected (Qi et al 2016). Four complex cations with 2:1 ligand to metal stoichiometry, $\mathbf{2}_{\mathbf{m G}}$, $\mathbf{2}_{\mathrm{pG}}, \mathbf{2}_{\mathrm{mA}}$ and $\mathbf{2}_{\mathrm{pA}}$, were chosen for the calculations, containing meta- or para-substituted triphenylphosphine ligand conjugated with glycine or alanine methyl ester, respectively and several conformations were considered for each of the cations. Relative energies of all optimized conformers of the ruthenium complex cations are collected in Table S2 (supplement). In addition, Cartesian coordinates of all computed structures are available as a single separate text file, for visualization and analysis. 
All calculated conformations contain aromatic stacking interactions ( $\pi-\pi$ stacking) between two different triphenylphosphine moieties, but with a different number of intramolecular hydrogen bonds between the ligands. In the case of $\mathbf{2}_{\mathbf{m G}}$, a representative conformation was generated with the aid of NMR experiments. NOESY experiment suggests position of amide protons in close proximity to the $\mathrm{C} 2$ and $\mathrm{C} 4$ atoms of the stacked phenylene subunit and also to the cymene ligand. This information indicates a "syn-Phe" orientation of two 1,3phenylene subunits forming a curved shape of the complex. Chloride ligand is directed outward the structure of complex.
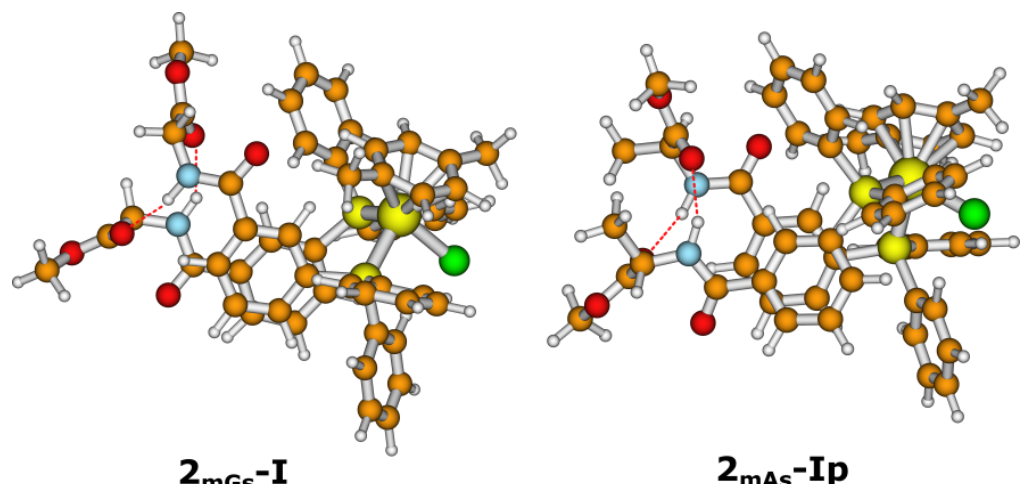

Figure 1. Optimized structures of $\mathbf{2}_{\mathrm{mGs}}-\mathrm{I}$ and $\mathbf{2}_{\mathrm{mAs}}-\mathbf{I p}$.

In context of intramolecular H-bonds between the ligands, both "syn-Phe" $\left(\mathbf{2}_{\mathbf{m G s}}\right)$ and "antiPhe" $\left(\mathbf{2}_{\mathbf{m G a}}\right)$ conformers can exist in a Herrick (I) or van Staveren (II) arrangement. Several conformers satisfying a "syn-Phe" arrangement were optimized and the lowest energy one shows a Herrick-type of hydrogen bonding motif $\left(\mathbf{2}_{\mathrm{mGs}}-\mathbf{I}\right)$. During our attempts to optimize $\mathbf{2}_{\mathrm{mGs}}-$ II conformer, reorganization of the hydrogen bonds occurred and the optimization ended up in "semi-Herrick" structure $\left(\mathbf{2}_{\mathbf{m G s}}-\mathbf{I b}\right)$ with only one NH-OCOMe hydrogen bond. We were able to optimize van Staveren conformer $\mathbf{2}_{\mathbf{m G s}}-\mathbf{I I}$ only with cymene ligand moved away from the stacked phenylene fragment. However, this structure strongly disagrees with the NOESY data and it is not likely present in solution. According to DFT calculations, $\mathbf{2}_{\mathbf{m G s}} \mathbf{}^{-}$ I is more stable than $\mathbf{2}_{\mathbf{m G a}}-\mathbf{I}, \mathbf{2}_{\mathbf{m G s}}-\mathbf{I I}$ and $\mathbf{2}_{\mathbf{m G a}}-\mathbf{I I}$ by 11,13 and $33 \mathrm{~kJ} \mathrm{~mol}^{-1}$, respectively. The lowest stability of conformer $\mathbf{2}_{\mathbf{m G a}}-\mathbf{I I}$, with only one hydrogen bond, is in good accordance with our previous results on ferrocene amino acids (Kirin et al 2006). These results additionally support the structural indications obtained by NOESY experiment.

In the case of $\mathbf{2}_{\mathbf{p G}}$, only one $\pi-\pi$ stacking motif with either Herrick or van Staveren hydrogen bonding scheme ( $\mathbf{2}_{\mathbf{p G}} \mathbf{- I}$ and $\mathbf{2}_{\mathbf{p G}}-\mathbf{I I}$, respectively) is possible. The calculations indicate the somewhat lower energy for $\mathbf{2}_{\mathbf{p G}^{-}} \mathbf{I}$ than for $\mathbf{2}_{\mathbf{p G}^{-}} \mathbf{I I}$ conformers (by ca $6 \mathrm{~kJ} \mathrm{~mol}^{-1}$, Table $\mathbf{S 2}$ ). Inspection of the geometrical parameters reveals significantly longer hydrogen bonds in $\mathbf{2}_{\mathbf{p G}^{-}}$ II than in $\mathbf{2}_{\mathbf{p G}} \mathbf{- I}(\mathrm{HB}$, above $2.0 \AA$ vs. $1.919 \AA)$ indicating origin of the energy difference.

The analogous conformers were also identified for the chiral L-alanine derivatives $\mathbf{2}_{\mathbf{m A s}}, \mathbf{2}_{\mathbf{m A a}}$ and $\mathbf{2}_{\mathbf{p A}}$. Only L-alanine derivatives were used for the calculations, to allow comparison with compounds experimentally prepared herein. The starting structures were generated from the glycine-based conformations by replacement of one $\mathrm{C}_{\alpha}$-proton with methyl group. Again, the most stable structure is characterized by "syn-Phe" arrangement of the stacked phenylene subunit with Herrick motif of hydrogen bonding ( $\left.\mathbf{2}_{\mathrm{mAs}}-\mathbf{I p}\right)$ with P-type of helical chirality. This result confirms preferred "syn-Phe" arrangement in both aminoacid bioconjugates. 
According to calculations, conformer $\mathbf{2}_{\mathrm{mAs}}-\mathbf{I p}$ is more stable than $\mathbf{2}_{\mathrm{mAs}}-\mathbf{I m}$ and $\mathbf{2}_{\mathrm{mAa}}-\mathbf{I m}$, by 20 and $18 \mathrm{~kJ} \mathrm{~mol}^{-1}$, respectively. Calculated lower stability of M- helical isomers of $\mathbf{2}_{\mathbf{m A s}}-\mathbf{I m}$ with respect to its $\mathrm{P}$ - diastereomer is ascribed to the arrangement of two methyl groups of alanine fragments. More stable structure (P-helical isomer) has these two methyl groups relatively close to each other $(\mathrm{d}=4.088 \AA)$. Although one could expect pronounced sterical repulsion, this is apparently not the case. It is somewhat surprising that $\mathbf{2}_{\text {mAs }}$-IIp lies only 8 $\mathrm{kJ} \mathrm{mol}^{-1}$ above $\mathbf{2}_{\mathrm{mAs}}-\mathrm{Ip}$ and it is more stable than $\mathbf{2}_{\mathrm{mAa}}-\mathbf{I m}$ in spite of reduced number of hydrogen bonds. The geometry of $\mathbf{2}_{\mathrm{mAs}}$-IIp is similar to $\mathbf{2}_{\mathbf{m G s}}$-II and, if we assume similar conformation of glycine and alanine derivatives, its presence in the solution is unlikely.

Finally, we shall briefly comment the structure of $\mathbf{2}_{\mathbf{p A}}$ complex. The lowest Gibbs energy was obtained for the Herrick-type complex $\mathbf{2}_{\mathbf{p A}}-\mathbf{I}$ and it is more stable than $\mathbf{2}_{\mathrm{pA}}-\mathbf{I I}$ by ca $11 \mathrm{~kJ}$ $\mathrm{mol}^{-1}$. Relative stabilities of two diastereomers (defined by P- and M- helicity) in comparison to the isomer $\mathbf{2}_{\mathrm{pA}}-\mathbf{I I}$ follows the same trend as for $\mathbf{2}_{\mathbf{m A s}}-\mathbf{I}$ derivative, P-helicity with the distance between the alanine methyl groups of $4.060 \AA$ is preferred over the M- isomer by 19 $\mathrm{kJ} \mathrm{mol}^{-1}$. The latter is even less stable than $\mathbf{2}_{\mathrm{pA}}-\mathrm{II}$ by $5 \mathrm{~kJ} \mathrm{~mol}^{-1}$.

Cytotoxicity. The cytotoxicity of ligands $\mathbf{L}$, as well as their $\mathbf{M L}$ and $\mathbf{M L}_{2}$ ruthenium complexes, was examined by MTT assay; ruthenium precursor di- $\mu$-chlorobis $[(p-$ cymene)chloro-ruthenium(II)] was included for comparison. For this purpose, the wellknown human cervical carcinoma cell line was used (HeLa), attested for screening of new compounds (Brozovic et al 2014, Cimbora-Zovko et al 2011). The $\mathrm{IC}_{50}$ value (the cytotoxicity of the compound expressed as the dose that reduced the survival of cells in $72 \mathrm{~h}$ MTT assay to $50 \%$ of the value obtained for control cells) was above $33.3 \mu \mathrm{M}$ for all ligands $\mathbf{L}$ and mono-ligand ruthenium complexes ML (Table S3). The ruthenium precursor was not toxic for the cells (also Table S3). However, the $\mathrm{IC}_{50}$ for bis-ligand ruthenium complexes $\mathbf{2}_{\mathrm{pG}}, \mathbf{2}_{\mathrm{pA}}, \mathbf{2}_{\mathrm{mG}}$ and $\mathbf{2}_{\mathrm{mA}}$ was 5-30 $\mu \mathrm{M}$, making them interesting for further examination as potential antitumor compounds (Table 1). The compound $\mathbf{2}_{\mathbf{p G}}$ was the least toxic and the $\mathbf{2}_{\mathbf{m A}}$ was the most toxic one. In order to avoid the solvent's toxicity, concentrations above 33.3 $\mu \mathrm{M}$ were not used. In that way, the highest concentration of the solvent in the well was under $0.5 \%$, which is not toxic for the HeLa cells.

Table 1. The cytotoxic activity of the compounds towards the HeLa cells $\left(\mathrm{IC}_{50} \boldsymbol{\mu M} \pm\right.$ SD), after $72 \mathbf{h}$ incubation. The cytotoxicity was measured by MTT assay. The experiments were repeated at least three times. Cp. $=$ Compound.

\begin{tabular}{|c|c|}
\hline $\mathbf{C p .}$ & $\mathbf{I C}_{\mathbf{5 0}} \pm \mathbf{S D}(\boldsymbol{\mu M})$ \\
\hline $\mathbf{2}_{\mathbf{p G}}$ & $30 \pm 3.2$ \\
\hline $\mathbf{2}_{\mathbf{p A}}$ & $15 \pm 3.7$ \\
\hline $\mathbf{2}_{\mathbf{m G}}$ & $16 \pm 2.5$ \\
\hline $\mathbf{2}_{\mathbf{m A}}$ & $5 \pm 2.7$ \\
\hline
\end{tabular}

The $\mathrm{IC}_{50}$ values of 5 to $30 \mu \mathrm{M}$ measured in HeLa cells for $\mathbf{M L}_{\mathbf{2}}$ complexes presented herein were similar to the value obtained under same experimental conditions for broadly used chemotherapeutic, cisplatin (Stojanovic et al 2013). Moreover, our results for additional tumor cell lines investigated are similar to the values previously obtained for breast carcinoma (MDA-MB-231) cells, treated with ruthenium complexes substituted with amino acid. The authors concluded that the amino acids do not significantly affect the activity of the 
complexes, but existence of the methyl groups in diamine increase their biological activity (Dos Santos et al 2016). The presence of triphenylphospine group seems to increment the antitumor properties of Ru complexes through intercalation in the DNA of the human acute promyelocytic leukemia (HL60) cells with $\mathrm{IC}_{50}$ value $5.2 \mu \mathrm{M}$ (Saez et al 2014).

One of the criterions necessary for a newly synthesized complex to be considered as a potential antitumor compound is low toxicity for normal cells and non-selective toxicity for different tumor cell lines. With the purpose to test this criterion, for further experiments we decided to use $\mathbf{2}_{\mathbf{m G}}$, due to its medium value of toxicity within the studied series of compounds. In order to examine the toxic capacity of $\mathbf{2}_{\mathbf{m G}}$ on different types of human tumor cells, the laryngeal carcinoma (HEp2), lung carcinoma (H460) and MDA-MB-231 cells were used. The collected data showed that $\mathbf{2}_{\mathbf{m G}}$ is similarly toxic to all examined cancer cell lines (Table 2). However, the $\mathrm{IC}_{50}$ of $\mathbf{2}_{\mathbf{m G}}$ for normal human cell line, keratinocytes, was far above the toxicity measured for all examined compounds; the same result was obtained for human fibroblasts (Table 2).

Table 2. The cytotoxic activity of the $2_{\mathrm{mg}}$ towards different tumor and two normal cell lines $\left(\mathrm{IC}_{\mathbf{5 0}} \boldsymbol{\mu M} \pm \mathbf{S D}\right)$, after $72 \mathrm{~h}$ incubation. The cytotoxicity was measured by MTT assay. The experiments were repeated at least three times. Cp. Compound.

\begin{tabular}{|l|l|l|l|l|l|l|}
\hline Cp. & HEp2 & H460 & HeLa & $\begin{array}{l}\text { MDA- } \\
\text { MB-231 }\end{array}$ & keratinocyte & fibroblast \\
\hline $\mathbf{2}_{\mathrm{mG}}$ & $14 \pm 4.7$ & $15 \pm 0.6$ & $16 \pm 2.5$ & $12.3 \pm 0.9$ & $>33.3$ & $>33.3$ \\
\hline
\end{tabular}

Potential biomacromolecule target evaluation. For the small bioactive molecules, determination of the main target within the living cell is a quite challenging and timeconsuming task, particularly for the structures presented herein, that contain DNA-targeting and protein-targeting features. In general, condensed aryl-Ru complexes are well known DNA binders (Gasser et al 2011); however, here presented structures do not contain condensed arenes necessary for DNA intercalation, nor several positive charges which could interact with DNA-backbone. Nevertheless, attached amino acid residues could form several H-bonds within DNA minor groove. On the other side, general spherical shape of the studied molecules is characterized by high hydrophobicity and thus could be nicely accommodated within hydrophobic pockets of well-known carrier proteins like serum albumins, within which amino acid residues from the studied small molecules could additionally contribute to the protein-substrate complex formation by H-bonding.

To facilitate the planning and the analysis of demanding cellular experiments in respect of the main target choice, we studied interactions of several Ru-complexes with model DNA (calf thymus, $c t$-DNA) and model protein: serum albumin (BSA), as the most abundant protein in blood plasma, that is responsible for the transport of many small molecules (among which are drugs and probes) (Otagiri et al 2016, Peters et al 1995). Experiments were performed in simple, well-defined conditions in cuvettes, at biologically relevant conditions (buffered $\mathrm{pH}$ 7) containing only studied Ru-complex (micromolar concentration) and biomacromolecule (ct-DNA or BSA).

The studied Ru-complexes did not stabilize ct-DNA against thermal denaturation nor changed the DNA CD spectrum, thus showing no measurable interaction (Figure S4). However, addition of Ru-complexes induced measurable changes in the BSA CD spectrum at 
240 - $290 \mathrm{~nm}$ (Figure S5), suggesting structural change in the protein chiral conformation; non-linear dependence of the CD change agreed well with a non-covalent binding event.

Furthermore, the intrinsic fluorescence of BSA significantly quenched upon Ru-complex addition. Multivariate analysis of the fluorimetric data by Specfit program (Gampp et al 1985, Maeder and Zuberbuhler 1990) revealed formation of 1:1 stoichiometry Rucomplex/BSA complex with micromolar affinity (Figures S6-S9). Intriguingly, non-linear dependence of the BSA CD spectrum changes at $260 \mathrm{~nm}$ (Figures S6-S9, Table 3) also supported similar binding affinity. Thus, results obtained suggest that the observed cytotoxic activity of compounds is not related to any interactions of complexes with the cellular DNA, but more likely with the protein target.

Table 3. Stability constants $\left(\log K_{s}\right)^{\mathrm{a}}$ and spectroscopic properties $(\Delta \mathrm{I})^{\mathrm{b}}$ of the complexes that ruthenium(II)-arene compounds formed with BSA protein, calculated according to the fluorimetric titrations (Na-cacodylate buffer, $\mathrm{c}=0.05 \mathrm{~mol} \mathrm{dm}^{-3}, \mathrm{pH}=7.0 ; \lambda_{\mathrm{exc}}=300 \mathrm{~nm} ; \lambda_{\mathrm{em}}=$ $\left.320-450 \mathrm{~nm}, \mathrm{c}(\mathbf{B S A})=5 \times 10^{-7} \mathrm{~mol} \mathrm{dm}^{-3}\right)$.

\begin{tabular}{|c|c|c|}
\hline $\mathbf{C p .}$ & $\Delta \mathbf{I}^{\mathbf{b}}$ & $\log \boldsymbol{K} \boldsymbol{s}^{\mathbf{a}}$ \\
\hline $\mathbf{2}_{\mathbf{p G}}$ & $-95 \%$ & $5.08 \pm 0.07$ \\
\hline $\mathbf{2}_{\mathbf{p A}}$ & $-48 \%$ & $5.99 \pm 0.07$ \\
\hline $\mathbf{2}_{\mathbf{m G}}$ & $-57 \%$ & $5.84 \pm 0.05$ \\
\hline $\mathbf{2}_{\mathbf{m A}}$ & $-34 \%$ & $6.50 \pm 0.09$ \\
\hline
\end{tabular}

a Processing of titration data by Specfit program (Gampp et al 1985, Maeder and Zuberbuhler 1990) gave best fit for 1:1 stoichiometry; for all titrations concentration range corresponded to ca. $20-80 \%$ complexation. ${ }^{\mathrm{b}}$ Changes in fluorescence of BSA induced by complex formation $\left(\Delta \mathrm{I}=\left(\mathrm{I}_{\text {lim}}-\mathrm{I}_{0}\right) \times 100 / \mathrm{I}_{0}\right.$; where $\mathrm{I}_{0}$ was calculated emission intensity of free compound and $\mathrm{I}_{\text {lim }}$ was emission intensity of a complex calculated by Specfit program.

A similar protein binding affinity was observed previously for RAPTA-C, the $\mathrm{Ru}$ containing antimetastatic agent, that accumulates at specific histone site on the nucleosome core. It seems that changing the ethylenediamine ligand of $[\mathrm{Ru}-\text { cymene-Cl }]^{+}$to phosphaadamantane $\left(+\mathrm{Cl}^{-}\right)$switches the adduct formation profile from primary targeting the DNA to targeting the proteins associated with chromatin (Adhireksan et al 2014).

Interestingly, strong correlation between the cytotoxic activity of compounds towards HeLa cells (Table 1) and the stability constants of compound-BSA complexes (Table 3) was observed. Specifically, compound $\mathbf{2}_{\mathbf{m A}}$ showed both the highest cytotoxic activity and the highest affinity for BSA constant, while $\mathbf{2}_{\mathbf{p G}}$ showed the lowest cytotoxicity and the lowest stability constant toward BSA among examined compounds. This correlation additionally supported protein as one of the main biological targets for ruthenium complexes in this study.

Cell cycle. Further, we were interested to investigate the mechanism of toxicity of bis-ligand ruthenium complexes and for this purpose we treated HeLa cells with one of them; $16 \mu \mathrm{M}$ $\mathbf{2}_{\mathbf{m G}}$ during 24-72 h. Upon flow cytometric analysis it was shown that $\mathbf{2}_{\mathbf{m G}}$ caused a slight time-dependent increase of cells in $\mathrm{S}$ phase of the cell cycle that is more visible in doseresponse of cells to $\mathbf{2}_{\mathbf{m G}}$ treatment, along with consequential decrease of cells in G0/G1 phase (Figure 2A). This was accompanied by the increase of the cells present in the SubG0/G1 
fraction, which represent the population of dead cells. The increase of the SubG0/G1 cell fraction was confirmed by the treatment of HeLa cells during $72 \mathrm{~h}$ with different concentrations of $\mathbf{2}_{\mathbf{m G}}$ (Figure 2B). Deconvolution algorithm (Watson pragmatic) was used for cell cycle data analysis. The results were confirmed by time- and dose-dependent cell cycle analysis upon treatment of HEp2 cells with $\mathbf{2}_{\mathbf{m G}}$, where increase of the cell amount in $\mathrm{S}$ phase of the cell cycle is more visible compared to the HeLa cells due to their higher sensitivity to $\mathbf{2}_{\mathrm{mG}}$ (Figure $\mathbf{S 1 1}$ ).
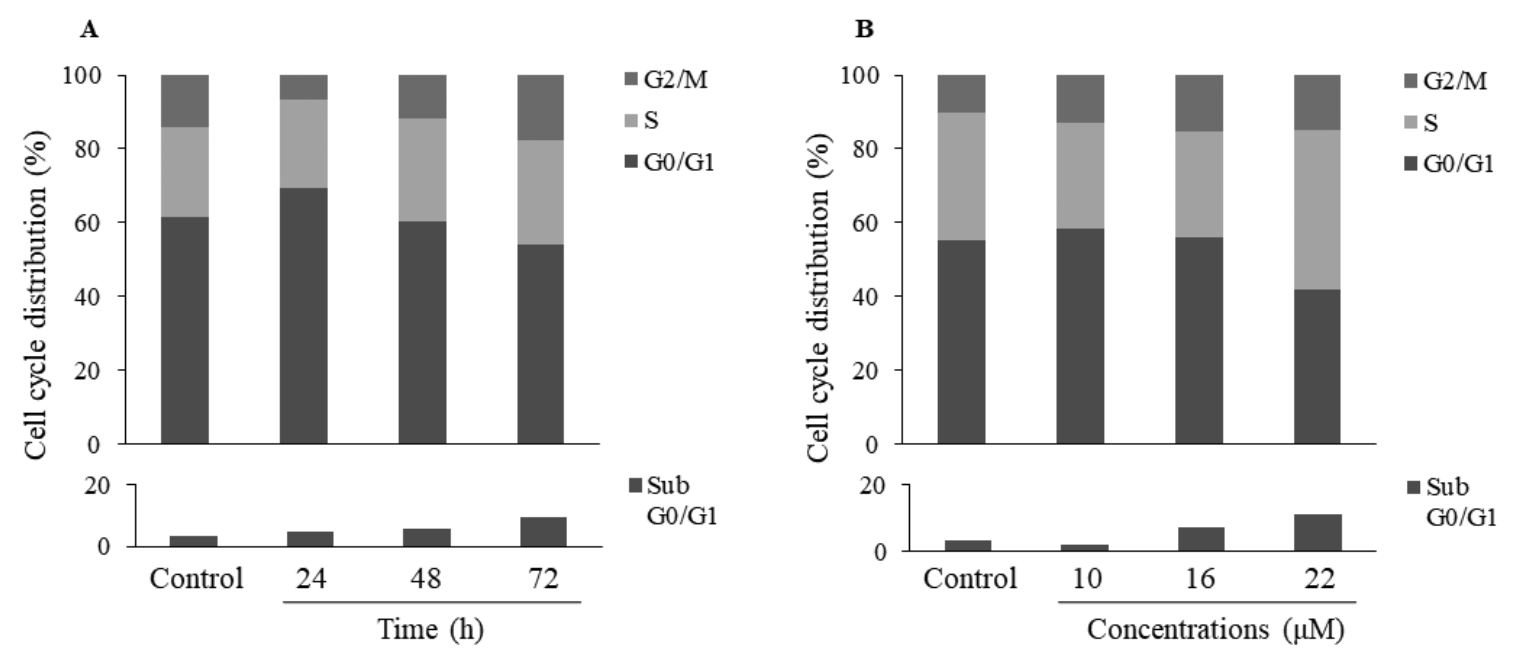

Figure 2. $\mathbf{2}_{\mathbf{m G}}$ compound induces increase of HeLa cells in $\mathrm{S}$ phase and consequential decrease of cells in G0/G1 phase of the cell cycle, and an increase of SubG0/G1 cell population. Cells were treated either with $16 \mu \mathrm{M} \mathbf{2}_{\mathrm{mG}}$ during $24-72 \mathrm{~h}$ (A) or with different concentrations of $\mathbf{2}_{\mathrm{mG}}$ during $72 \mathrm{~h}$ (B). The data of one from three performed experiments are shown.

Cell death. Necrosis and apoptosis are two major types of cell death. Inappropriate regulation of both processes can result in several diseases, including cancer (Nikoletopoulou et al 2013). With the purpose to distinguish between necrotic and apoptotic cell death, HeLa cells were treated with different concentrations of $\mathbf{2}_{\mathbf{m G}}$ during 24-72 $\mathrm{h}$ and then stained with Annexin V-FITC and propidium iodide (PI). As shown in Figure 3, the percentage of PI negative (-)/Annexin V-FITC positive $(+)$ and PI+/Annexin V-FITC+ cells increased with the time (Figure 3A) as well as with the dose (Figure 3B) indicating apoptosis as mode of $\mathbf{2}_{\mathbf{m G}}$ induced cell death. Starved and/or heat shocked cells were always used as compensation controls for Annexin V-FITC and PI staining for all performed experiments.

One of the most common signaling cascades involved in apoptosis is the activation of a highly specialized family of cysteinyl-aspartate proteases (caspases), which are usually present in the inactive zymogen forms. Once activated, caspases initiate cell death by cleaving and thus activating effector caspases, which drive the process of apoptosis (Li and Yuan 2008). Caspase mediated apoptotic cell death is accomplished through the cleavage of several key proteins required for cellular functioning and survival (Fischer et al 2003). PARP-1 is one of the several known cellular substrates of caspases. Cleavage of PARP-1 by caspases is considered to be the hallmark of apoptosis (Kaufmann et al 1993, Tewari et al 1995). In order to test our conclusion that $\mathbf{2}_{\mathbf{m G}}$ induces apoptosis, formulated based on the data from Figure 3A and 3B, we treated HeLa cells with $22 \mu \mathrm{M}$ concentration of the complex 
during 24-72 $\mathrm{h}$. Our data showed that $\mathbf{2}_{\mathbf{m G}}$ does not induce PARP cleavage, which was detected in HeLa cells treated $72 \mathrm{~h}$ with $5 \mu \mathrm{M}$ cisplatin (cDDP) used as a positive control (Figure 3C). Moreover, the pre-treatment of HeLa cells with pan caspase inhibitor that irreversibly binds to the catalytic site of caspase proteases and can inhibit the induction of apoptosis, carbobenzoxy-valyl-alanyl-aspartyl-[O-methyl]-fluoromethylketone (Z-VAD), did not protect cells from $\mathbf{2}_{\mathbf{m G}}$ induced cell death (Figure 3D). Additionally, the caspase independent cell death was confirmed upon specifically measuring caspase 3 and 7 activity in the HeLa cells treated 48 and $72 \mathrm{~h}$ with $22 \mu \mathrm{M} \mathbf{2}_{\mathbf{m G}}$ (Figure S12A), and by two hours pretreatment of additional cell system, HEp2 cells, with Z-VAD prior to treatment with $\mathbf{2}_{\mathbf{m G}}$ (Figure S12B). The activity of caspase $3 / 7$ upon $\mathbf{2}_{\mathbf{m G}}$ treatment was not detected (Figure S12A) and Z-VAD did not increase survival of HEp2 cell line upon $\mathbf{2}_{\mathbf{m G}}$ treatment (Figure S12B). The fact that we did not detect cleavage of PARP and that Z-VAD did not prevent $\mathbf{2}_{\mathbf{m G}}$ induced cell death brought us to the hypothesis that $\mathbf{2}_{\mathbf{m G}}$ probably induces caspase independent cell death. The pre-treatment of cells with inhibitor of necroptosis, necrostatin-1 (Vandenabeele et al 2013), did not protect cells from death (Figure S13), excluding this type of cell death from the involvement in the $\mathbf{2}_{\mathbf{m G}}$ toxicity.
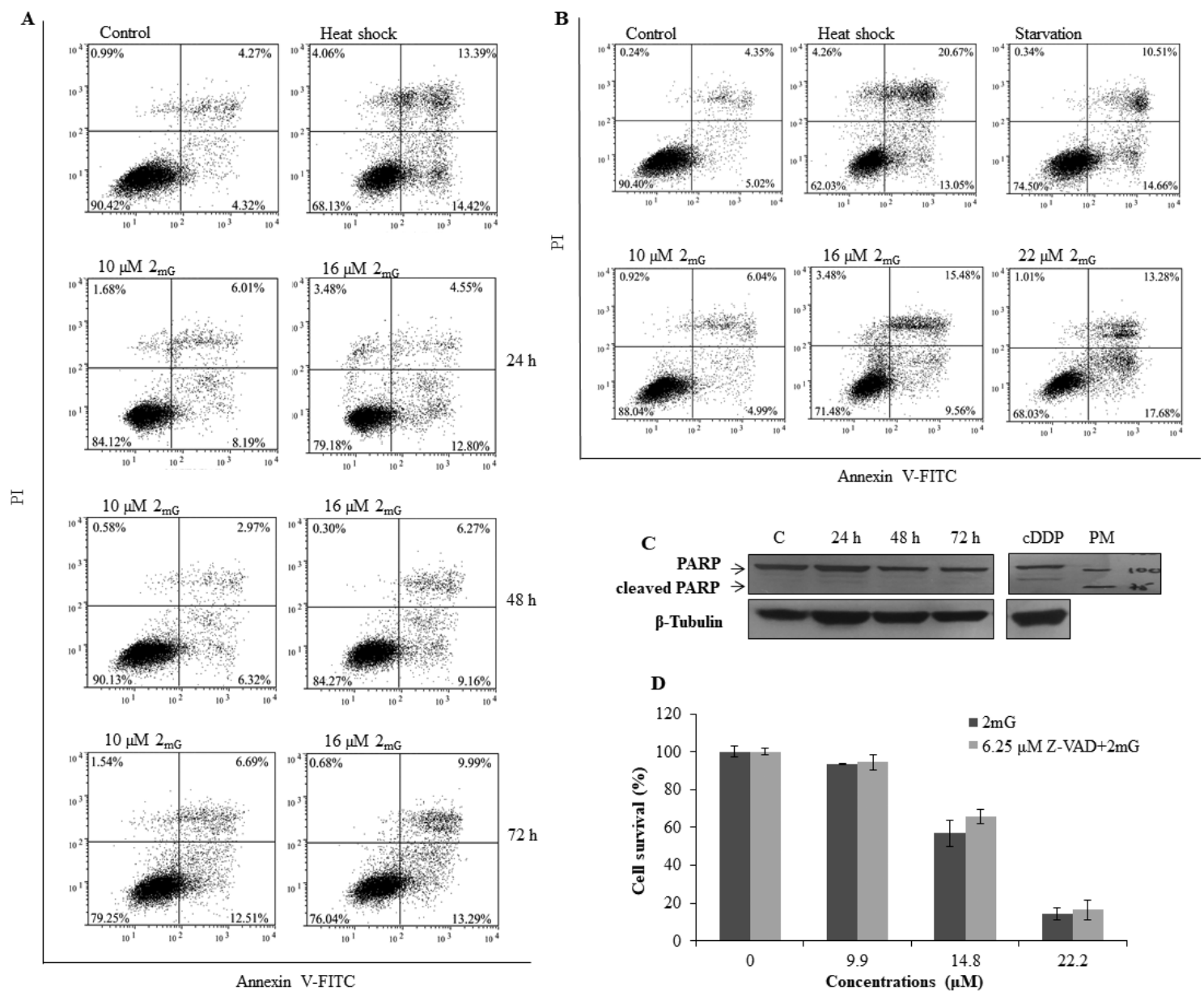

Figure 3. $2_{\mathrm{mG}}$ compound induces cell death in HeLa cells. Cells were treated either with 10 or $16 \mu \mathrm{M} \mathbf{2}_{\mathrm{mG}}$ for $24-72 \mathrm{~h}$ (A) or 10,16 and $22 \mu \mathrm{M} \mathbf{2}_{\mathbf{m G}}$ for $72 \mathrm{~h}$ (B). The cell death was measured by flow cytometry. The one of three performed experiments for each set of conditions is shown. Starved ( $24 \mathrm{~h}$ cell growth without serum) or heat shocked (10 min, 56 $\left.{ }^{\circ} \mathrm{C}\right)$ cells were always used as a compensation Annexin V-FITC/PI controls. PI-/Annexin V- 
FITC- cells (lower left quadrant), PI-/Annexin V-FITC+ cells (lower right quadrant), $\mathrm{PI}+/$ Annexin V-FITC+ cells (upper right quadrant). Cleavage of PARP was detected by Western blot. $\beta$-Tubulin was used as a loading control. One out of three performed experiments is presented. HeLa cells treated with $5 \mu \mathrm{M}$ cisplatin (cDDP) during $72 \mathrm{~h}$ were used as positive control for PARP cleavage (C). HeLa cells were pre-treated for two hours with $6.25 \mu \mathrm{M}$ Z-VAD prior to treatment with different concentrations of $\mathbf{2}_{\mathbf{m G}}$. The cell survival was measured $72 \mathrm{~h}$ after the treatment by MTT assay. The experiments were repeated at least three times $(\mathrm{D})$.

From the literature is known that cells can enter autophagy prior to dying (Dikic and Elazar 2018, Levine and Kroemer 2008). Autophagy is a homoeostatic cellular process regulating protein and organelle turnover by lysosomal destruction (Levine and Kroemer 2008). The treatment of HeLa cells with $22 \mu \mathrm{M} \mathbf{2}_{\mathbf{m G}}$ during $24-72 \mathrm{~h}$ induced increase in Beclin-1, which is accompanied with the increase of proteolytic derivate LC3-II visible upon $72 \mathrm{~h}$ treatment (Figure 4A). This is in line with the fact that Beclin-1 is involved in autophagosome formation and LC3 is located in the cytosol (LC3-I) or in autophagosomal membranes (LC3II). LC3-II may thus be used to estimate the abundance of autophagosomes prior to their destruction through fusion with lysosomes (Klionsky et al 2012). With the intention to verify the possible role of autophagy in $\mathbf{2}_{\mathbf{m G}}$ induced cell death, the cells were pretreated with bafilomycin A (BAF A), which is a known inhibitor of the late phase of autophagy (Klionsky et al 2012), and then treated with different concentrations of $\mathbf{2}_{\mathbf{m G}}$. BAF A decreased the toxic effect of $\mathbf{2}_{\mathbf{m G}}$ (Figure $\mathbf{4 B}$ ) pointing towards the conclusion that $\mathbf{2}_{\mathbf{m G}}$ induces autophagy. Additional inhibitor of autophagy, 3-methyladenine (3-MA), which is an inhibitor of phosphatidylinositol 3-kinases (PI3K), decreased the toxicity of $\mathbf{2}_{\mathbf{m G}}$ as well (Figure S11). PI3K plays an important role in many biological processes, including controlling the activation of the mechanistic target of rapamycin (mTOR), a key regulator of autophagy (Wu et al 2010). Autophagy as the mode of cell death upon $\mathbf{2}_{\mathbf{m G}}$ treatment was confirmed in HEp2 cell line by MTT and SRB assays (Figure S14A and S14B). The obtained data imply the autophagy as a part of $\mathbf{2}_{\mathbf{m G}}$ triggered caspase independent programmed cell death. There are multiple caspase-dependent and -independent mechanisms by which classical features of programmed cell death are mediated (Liu et al 2003). Further investigation is needed to understand $\mathbf{2}_{\mathbf{m G}}$ triggered caspase independent programmed cell death and the correlation of autophagy with it. 
$\mathbf{A}$

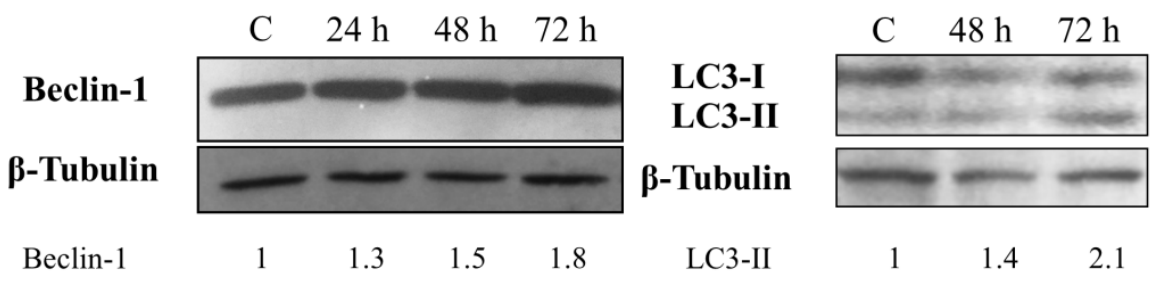

B

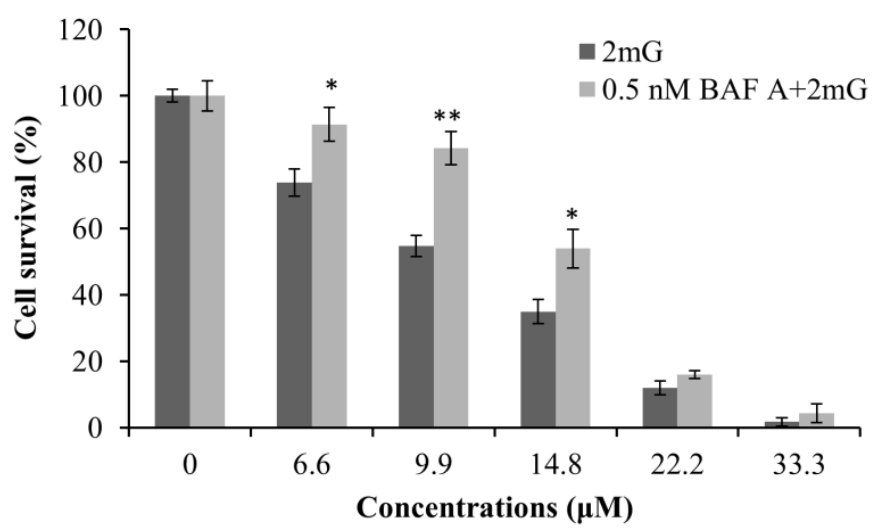

Figure 4. $2_{\mathrm{mG}}$ compound induces autophagy in HeLa cells. Cells were treated with $22 \mu \mathrm{M}$ $\mathbf{2}_{\mathbf{m G}}$. The expression of Beclin-1, LC3-I and LC3-II was detected by Western blot. $\beta$-Tubulin was used as a loading control. Data obtained with $\mathrm{d}$ Đensitometric analysis are expressed as a ratio between examined Beclin-1 or LC3-II and $\beta$-Tubulin. Non-treated cells were set as 1.0. One out of three performed experiments for each protein is presented (A). HeLa cells were pre-treated for two hours either with $6.25 \mu \mathrm{M}$ Z-VAD or with $0.5 \mathrm{nM}$ BAF A prior to treatment with different concentrations of $\mathbf{2}_{\mathbf{m G}}$. The cell survival was measured $72 \mathrm{~h}$ after the treatment by MTT assay. The experiments were repeated at least three times. Significance was determined between the $\mathbf{2}_{\mathbf{m G}}$ only and either Z-VAD or BAF A pre-treated and $\mathbf{2}_{\mathbf{m G}}$ treated cells $(*, \mathrm{P}<0.05 ; * *, \mathrm{P}<0.01)(\mathrm{B})$.

The role of glutathione. The fact that cancer cells are generally growing and multiplying much faster than normal healthy cells and have a rapid production of new biomolecules, makes them more vulnerable to compounds such as the one investigated. Also, tumor cells produce a reductive environment due to the increased metabolic rate, feature that opens the possibility to activate ruthenium complexes solely at the site of cancerous cells (Antonarakis and Emadi 2010). The tripeptide GSH has the capacity to bind different metal-based drugs (Brozovic et al 2010). We were interested in the possibility that GSH is involved in detoxification of investigated compounds due to their capacity to bind proteins (Table 3 ). For that purpose, HeLa cells were either pre-treated with specific inhibitor of GSH synthesis, $0.01 \mu \mathrm{g} / \mathrm{mL}$ buthionine sulfoximine (BSO) overnight or with precursor of GSH synthesis, 5 $\mathrm{mM}$-acetylcysteine (NAC) for two hours prior to treatment with $\mathbf{2}_{\mathbf{p G}}, \mathbf{2}_{\mathbf{p A}}, \mathbf{2}_{\mathbf{m G}}$ or $\mathbf{2}_{\mathbf{m A}}$. The conditions used were tested previously to be effective (Brozovic et al 2008, Brozovic et al 2014). The obtained data showed decreased survival of HeLa cells in the case when GSH was depleted by BSO and increased survival of cells in the case when the level of GSH was increased due to the NAC (Figure 5A-D). Similar results were obtained for all examined bis- 
ruthenium complexes, showing the importance of GSH in the toxicity of examined compounds.

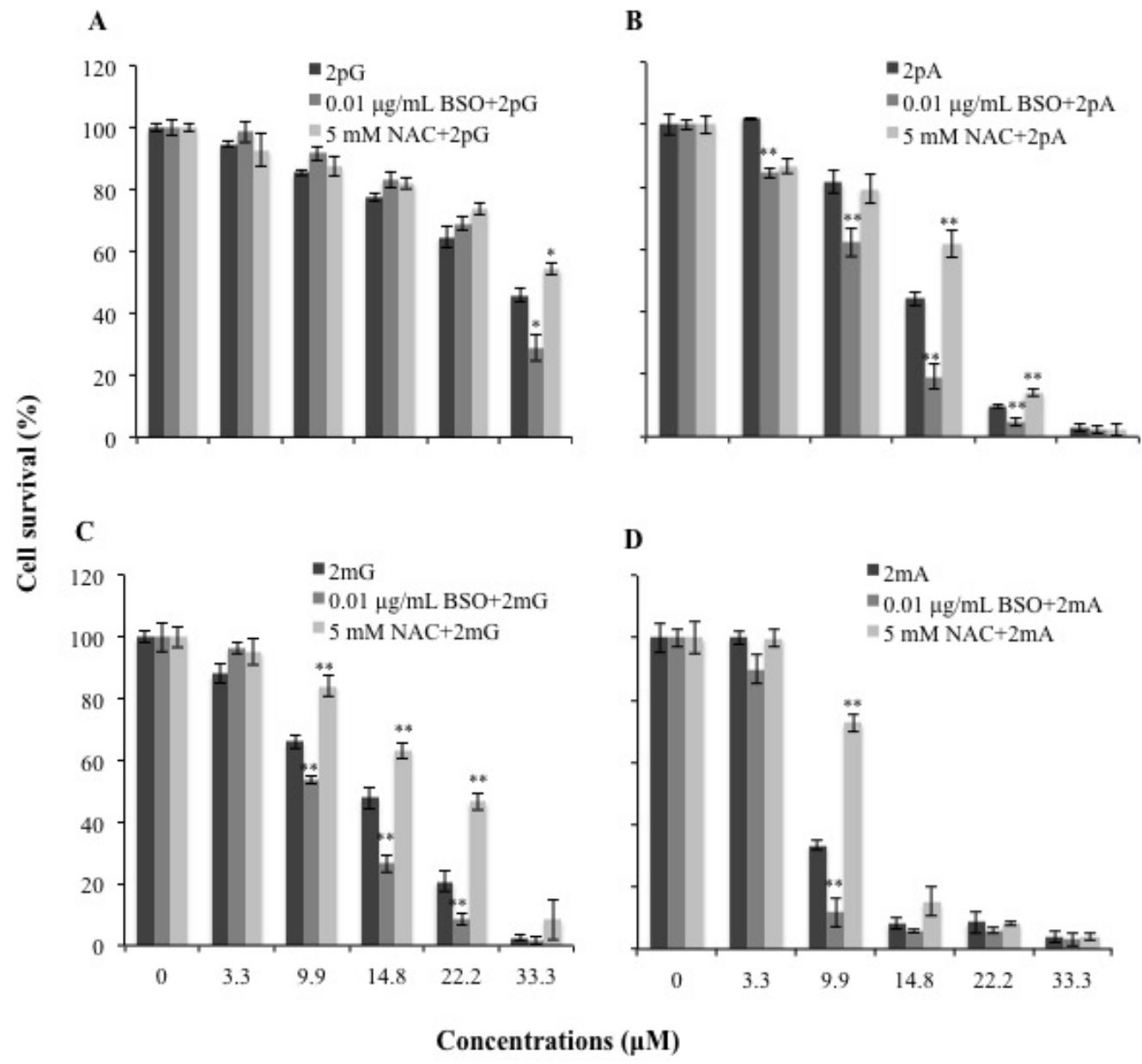

Figure 5. Glutathione reduced the toxicity of $\mathbf{2}_{\mathrm{pG}}, \mathbf{2}_{\mathrm{pA}}, \mathbf{2}_{\mathrm{mG}}$ and $\mathbf{2}_{\mathrm{mA}}$. HeLa cells were pretreated either with $0.01 \mu \mathrm{g} / \mathrm{mL}$ BSO (overnight) or with $5 \mathrm{mM} \mathrm{NAC}(2 \mathrm{~h})$ prior to treatment with different concentrations of $\mathbf{2}_{\mathbf{p G}}$ (A), $\mathbf{2}_{\mathbf{p A}}$ (B), $\mathbf{2}_{\mathbf{m G}}$ (C) or $\mathbf{2}_{\mathbf{m A}}$ (D). The cell survival was measured $72 \mathrm{~h}$ after the treatment by MTT assay. The experiments were repeated at least three times. Significance was determined between the $\mathbf{2}_{\mathbf{m G}}$ only and either BSO or NAC pretreated and $\mathbf{2}_{\mathrm{pG}} / \mathbf{2}_{\mathrm{pA}} / \mathbf{2}_{\mathrm{mG}} / \mathbf{2}_{\mathrm{mA}}$ treated cells $(*, \mathrm{P}<0.05 ; * *, \mathrm{P}<0.01)$.

Since GSH is one of the major endogenous antioxidants, we next measured the formation of reactive oxygen species (ROS) upon treatment of HeLa cells with $\mathbf{2}_{\mathbf{m G}}$. We were not able to detect increased formation of ROS (Figure S12A and S12B) by flow cytometry, what is in line with our prediction that this type of chemical structure does not activate the formation of ROS. Therefore, we concluded that GSH probably has a role, not as an antioxidant, but rather as a detoxification mechanism in the cells. This hypothesis was confirmed with pre-treatment of cells with two antioxidant compounds, tempol and trolox, which did not protect cells from $\mathbf{2}_{\mathbf{m G}}$ toxicity (Figures S13A and S13B). The combination treatment of HeLa cells with well accepted inhibitor of glutathione S-transferase (GST) (a detoxification enzymes that catalyze the conjugation of GSH to a wide variety of endogenous and exogenous electrophilic compounds) (Townsend and Tew 2003), 5 or $7.5 \mu \mathrm{g} / \mathrm{mL}$ ethacrynic acid (ETA) (Osmak et al 1998), decreased cell survival compared with cells treated with $\mathbf{2}_{\mathbf{m G}}$ only (Figure 6A). The 
data implied enzymatically-regulated formation of detoxification complex between GSH and $\mathbf{2}_{\mathbf{m G}}$. Moreover, pre-treatment of HeLa cells with probenecid, the inhibitor of MRP/GS-X pumps (Zaman et al 1995), decreased survival of cells compared to the ones treated only with $\mathbf{2}_{\mathbf{m G}}$ (Figure 6B). These results indicate efflux of the formed conjugates out of the cells and the detoxification role of GSH.


Figure 6. Glutathione probably forms conjugates with the $\mathbf{2}_{\mathrm{mG}}$. HeLa cells were pretreated either with 5 or $7.5 \mu \mathrm{g} / \mathrm{mL}$ ETA (A) or with 0.2 or $0.625 \mathrm{mM}$ probenecid (B) prior to treatment with different concentrations of $\mathbf{2}_{\mathbf{m G}}$. The cell survival was measured $72 \mathrm{~h}$ after by MTT assay. The experiments were repeated at least three times. Significance was determined between the $\mathbf{2}_{\mathbf{m G}}$ only and either ETA or probenecid pre-treated and $\mathbf{2}_{\mathbf{m G}}$ treated cells $(*, \mathrm{P}<$ $0.05 ; * *, \mathrm{P}<0.01)$.

Cellular accumulation. Further we were interested to check the correlation between cellular accumulations of investigated compounds with their toxicity (Pongratz et al 2004, Puckett et al 2010). The HeLa cells were treated with $150 \mu \mathrm{M} \mathbf{2}_{\mathbf{p G}}, \mathbf{2}_{\mathbf{p A}}, \mathbf{2}_{\mathbf{m G}}$ or $\mathbf{2}_{\mathbf{m A}}$ during 2-6 h and analyzed by high-resolution inductively coupled plasma mass spectrometry (HR ICPMS). Our results showed that the amount of ruthenium in the cells is the highest upon treatment with $\mathbf{2}_{\mathbf{p A}}$ and the lowest upon treatment with $\mathbf{2}_{\mathbf{m G}}$ (Figure 7A). The obtained data were further confirmed by investigation of dose-dependent accumulation of ruthenium for all compounds (Figure 7B). The data also showed that the amount of ruthenium does not correlate with toxicity of each compound (Table 1). In a line with that, compound $\mathbf{2}_{\mathbf{m G}}$, used for most of the testing, was well chosen since it exhibits significant toxicity (Table 1) with the lowest amount of ruthenium in the cell. The most likely reasons, which need further investigation, could be different structural features of the compounds, which regulate different cellular uptake or even different capacity of GSH to detoxify them. The fact that hydrophobicity is an important pharmacological feature, closely related to the drug uptake and efflux, and the fact that phenyl group is hydrophobic (Liu et al 2011) could explain why phenyl group in paraposition $\left(\mathbf{2}_{\mathbf{p A}}\right)$ correlates with accumulation and in meta-position $\left(\mathbf{2}_{\mathbf{m A}}\right)$ with toxicity. By using the same model system, HeLa cells, it was shown that anti-metastatic effect of NAMI$A$ is unrelated to the penetration of the compound in the cell (Sava et al 2004). The extent of ruthenium uptake of three complexes did not correlate with their different cytotoxicity in ovarian cancer A2780 cell line (Bugarcic et al 2008, Komor and Barton 2013). 

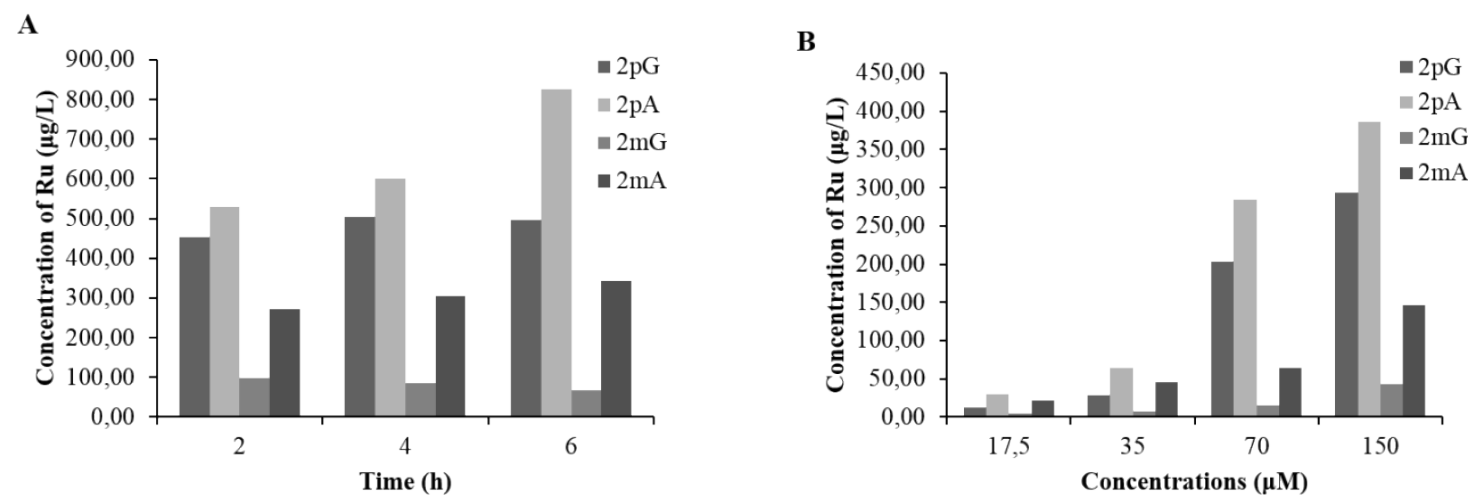

Figure 7. The accumulation of $\mathbf{2}_{\mathrm{pG}}, \mathbf{2}_{\mathrm{pA}}, \mathbf{2}_{\mathrm{mG}}$ and $\mathbf{2}_{\mathrm{mA}}$ in HeLa cells is different. The HeLa cells were either treated with $150 \mu \mathrm{M}$ concentration of $\mathbf{2}_{\mathrm{pG}}, \mathbf{2}_{\mathrm{pA}}, \mathbf{2}_{\mathrm{mG}}$ or $\mathbf{2}_{\mathrm{mA}}$ from two to six hours (A) or with 17.5-150 $\mu \mathrm{M}$ during two hours (B). The cells were collected at the indicated time points and the amount of ruthenium was measured by HR ICPMS. Two out of four independent experiments are presented.

\section{Conclusions.}

In this study, six triphenylphosphine ligands (L) conjugated to amino acids and ten ruthenium(II)-cymene complexes thereof, including both $\mathbf{M L}$ and $\mathbf{M L}_{\mathbf{2}}$ stoichiometry, were prepared, purified and characterized by NMR $\left({ }^{1} \mathrm{H},{ }^{13} \mathrm{C},{ }^{31} \mathrm{P}\right), \mathrm{CD}$ and $\mathrm{MS}$ techniques and DFT calculations (wB97xD, gas phase). Cytotoxicity screening using human cervical carcinoma (HeLa) cells and MTT assay revealed the $\mathrm{IC}_{50}$ value of 5-30 $\mu \mathrm{M}$ for all examined $\mathbf{M L}_{2}$ complexes ( $\mathbf{2}_{\mathrm{pG}}, \mathbf{2}_{\mathrm{pA}}, \mathbf{2}_{\mathrm{mG}}$ and $\left.\mathbf{2}_{\mathrm{mA}}\right)$, while for all other compounds ( $\mathbf{L}, \mathbf{M L}$ and $\mathrm{Ru}$ precursor) the $\mathrm{IC}_{50}$ values were above $33.3 \mu \mathrm{M}$. Additional analysis performed with $\mathbf{2}_{\mathbf{m G}}$ showed similar toxicity on several other human carcinomas (laryngeal carcinoma HEp2, lung carcinoma H460, and breast carcinoma MDA-MB-231), but no toxicity for normal human cell lines (both keratinocytes and fibroblasts). Studies of non-covalent interactions (thermal melting, $\mathrm{CD}$ and fluorescence titrations) of ruthenium complexes with $c t$-DNA and BSA, as model systems for potential biomolecular targets, revealed no measurable interaction with DNA, but a micromolar affinity for proteins. Interestingly, bioconjugates with lower $\mathrm{IC}_{50}$ values showed higher binding affinities towards BSA.

Further evaluation of biological activity showed that $\mathbf{2}_{\mathbf{m G}}$ increased cells in $\mathrm{S}$ phase and subsequently decreased them in G1 phase of the cell cycle, what was followed by increase of the SubG0/G1 cell fraction. Staining with Annexin V-FITC and PI indicated programed cell death as a mode of $\mathbf{2}_{\mathbf{m G}}$ action. Caspase independent cell death was deduced from (a) the lack of PARP-1 cleavage and (b) existence of cell death despite the Z-VAD presence. In addition, a similar effect obtained by pre-treatment of cells with necrostatin- 1 indicated absence of necroptosis, as another possible mode of cell death. Increase of Beclin-1 as well as LC3-II expression indicated that $\mathbf{2}_{\mathbf{m G}}$ induced autophagy as part of cell death. Moreover, the autophagy inhibitors BAF-A and 3-MA decreased the toxic effect of $\mathbf{2}_{\mathbf{m G}}$, confirming the involvement of autophagy in its toxic effect.

Importance of GSH in the cell response to all $\mathbf{M L} \mathbf{L}_{\mathbf{2}}$ complexes $\left(\mathbf{2}_{\mathbf{p G}}, \mathbf{2}_{\mathbf{p A}}, \mathbf{2}_{\mathbf{m G}}\right.$ and $\left.\mathbf{2}_{\mathbf{m A}}\right)$ was shown using BSO and NAC, revealing decreased (BSO) and increased (NAC) survival, respectively. Detoxification role of GSH was suggested by the absence of ROS formation and 
no effect of two antioxidants, tempol and trolox, on cell survival. On the other hand, increased survival was detected when ethacrynic acid was used, indicating detoxification role of GSH. This was further confirmed with inhibition of $\mathrm{GSH}-\mathbf{2}_{\mathbf{m G}}$ conjugates efflux by probenecid, which had the same effect on cell survival. Cellular uptake, analyzed by HR ICPMS, showed different dose- and time-dependent cellular accumulation of the studied complexes. It is particularly interesting to mention that para-substituted complexes $\left(\mathbf{2}_{\mathbf{p G}}\right.$ and $\left.\mathbf{2}_{\mathrm{pA}}\right)$ are characterized by higher uptake into the cell, while meta-substituted complexes $\left(\mathbf{2}_{\mathbf{m G}}\right.$ and $\left.\mathbf{2}_{\mathbf{m A}}\right)$ are more toxic.

Here, we described for the first time newly synthesized ruthenium complexes which are causing non oxidative protein damage and trigger autophagy, and also induce cells protection mechanism through GSH led detoxification. Taken together, $\mathbf{M L}_{\mathbf{2}}$ complexes deserve further investigation as potential chemotherapeutic agents for different types of cancer.

\section{Experimental.}

General. Reactions were carried out in ordinary glassware and chemicals were used as purchased from commercial suppliers without further purification. Reactions were monitored by TLC on Silica Gel 60 F254 plates and detected with UV lamp (254 nm); organic compounds were purified using automated flash chromatography equipped with a UV detector $(254 \mathrm{~nm})$ and prepacked silica columns. NMR spectra were obtained on spectrometer operating at 300.13 or $600.13 \mathrm{MHz}$ for ${ }^{1} \mathrm{H}, 242.92 \mathrm{MHz}$ for ${ }^{31} \mathrm{P}$ and 150.92 $\mathrm{MHz}$ for ${ }^{13} \mathrm{C}$ nuclei. Mass spectra were measured on a HPLC-MS system coupled with 6410 triple-quadrupole mass spectrometer, operating in a positive ESI mode. High-resolution mass spectra were obtained on a MALDI TOF-TOF instrument using a CHCA matrix. The CD spectra were recorded on a JASCO J-810 spectropolarimeter equipped with Peltier thermostat, using $1 \mathrm{~cm}$ Suprasil quartz cells with a scanning speed of $200 \mathrm{~nm} \mathrm{~min}{ }^{-1}$. The buffer background was subtracted from each spectrum, while each spectrum was a result of three accumulations. The electronic absorption spectra of ruthenium complexes and thermal melting experiments were measured on a Varian Cary 100 Bio spectrometer. Fluorescence titrations were recorded on Varian Cary Eclipse fluorimeter. UV-Vis and fluorescence spectra were recorded using appropriate $1 \mathrm{~cm}$ path quartz cuvettes.

Ligands, general procedure. The corresponding 4-(Diphenylphosphino)benzoic acid (1 mmol) was dissolved in dichloromethane $(50 \mathrm{~mL})$. $\mathrm{HOBt} \times \mathrm{H}_{2} \mathrm{O}(1 \mathrm{mmol})$, TBTU $(1 \mathrm{mmol})$ and DIPEA $(0.5 \mathrm{~mL}, 4 \mathrm{mmol})$ were added and the mixture was left stirring at room temperature. After $1 \mathrm{~h}$ methyl ester $(1 \mathrm{mmol})$ was added and the mixture was left stirring for the indicated period. After that, the reaction mixture was washed with $\mathrm{NaHCO}_{3}(3 \times 50 \mathrm{~mL}$, sat. aq.) and subjected to flash chromatography (hexane/EtOAc).

$\mathbf{P h}_{2} \mathbf{P}-\mathbf{p} \mathbf{C}_{6} \mathbf{H}_{4}$-CO-Gly-OMe, $\mathbf{L}_{\mathbf{p G}}$; (Kokan and Kirin 2012). 4-(Diphenylphosphino)benzoic acid $(301.6 \mathrm{mg}, 1 \mathrm{mmol})$, dichloromethane $(50 \mathrm{~mL})$, HOBt $\times \mathrm{H}_{2} \mathrm{O}(135.5 \mathrm{mg}, 1 \mathrm{mmol})$, TBTU (322.1 mg, $1 \mathrm{mmol})$, DIPEA (0.5 mL, $4 \mathrm{mmol})$ and $\mathrm{H}-\mathrm{Gly}-\mathrm{OMe} \times \mathrm{HCl}(129.4 \mathrm{mg}, 1$ mmol), $72 \mathrm{~h}$. Yield: $332.1 \mathrm{mg}(88 \%) .{ }^{1} \mathrm{H}$ NMR $\left(300.13 \mathrm{MHz}, \mathrm{CDCl}_{3}\right) \delta / \mathrm{ppm}: 7.75$ (dd, $2 \mathrm{H}$, $\left.J_{1}=8.5 \mathrm{~Hz}, J_{2}=1.5 \mathrm{~Hz}\right), 7.26-7.37(\mathrm{~m}, 12 \mathrm{H}), 6.62(\mathrm{t}, 1 \mathrm{H}, J=4.5 \mathrm{~Hz}), 4.25(\mathrm{~d}, 2 \mathrm{H}, J=5$ $\mathrm{Hz}), 3.8(\mathrm{~s}, 3 \mathrm{H})$.

$\mathbf{P h}_{2} \mathbf{P}-\mathbf{p} \mathbf{C}_{6} \mathbf{H}_{4}$-CO-Ala-OMe, $\mathbf{L}_{\mathbf{p A}}$; (Kokan et al 2014). 4-(Diphenylphosphino)benzoic acid (302.3 mg, $1 \mathrm{mmol})$, dichloromethane $(50 \mathrm{~mL}), \mathrm{HOBt} \times \mathrm{H}_{2} \mathrm{O}(134.2 \mathrm{mg}, 1 \mathrm{mmol})$, TBTU 
(320.3 mg, $1 \mathrm{mmol})$, DIPEA (0.5 mL, $4 \mathrm{mmol}), \mathrm{H}-\mathrm{Ala}-\mathrm{OMe} \times \mathrm{HCl}(132.1 \mathrm{mg}, 1 \mathrm{mmol}), 24$ h. Yield: $296.7 \mathrm{mg}(76 \%) .{ }^{1} \mathrm{H}$ NMR $\left(300.13 \mathrm{MHz}, \mathrm{CDCl}_{3}\right) \delta / \mathrm{ppm}: 7.73-7.76(\mathrm{~m}, 2 \mathrm{H}), 7.26-$ $7.37(\mathrm{~m}, 12 \mathrm{H}), 6.70(\mathrm{~d}, 1 \mathrm{H}, J=7 \mathrm{~Hz}), 4.75-4.84(\mathrm{~m}, 1 \mathrm{H}), 3.79(\mathrm{~s}, 3 \mathrm{H}), 1.51(\mathrm{~d}, 3 \mathrm{H}, J=7 \mathrm{~Hz})$.

$\mathbf{P h}_{2} \mathbf{P}-\boldsymbol{m C}_{6} \mathbf{H}_{4}$-CO-Gly-OMe, $\mathbf{L}_{\mathbf{p G}}$. 3-(Diphenylphosphino)benzoic acid (305.5 $\mathrm{mg}, 1 \mathrm{mmol}$ ), dichloromethane $(50 \mathrm{~mL})$, HOBt $\times \mathrm{H}_{2} \mathrm{O}(136.7 \mathrm{mg}, 1 \mathrm{mmol})$, TBTU $(322.3 \mathrm{mg}, 1 \mathrm{mmol})$, DIPEA (0.5 mL, $4 \mathrm{mmol}), \mathrm{H}-\mathrm{Gly}-\mathrm{OMe} \times \mathrm{HCl}(127.2 \mathrm{mg}, 1 \mathrm{mmol}), 24 \mathrm{~h}$. Yield: $291.8 \mathrm{mg}$ (77 \%). ${ }^{1} \mathrm{H}$ NMR (300.13 MHz, $\left.\mathrm{CDCl}_{3}\right) \delta / \mathrm{ppm}: 7.75-7.82(\mathrm{~m}, 2 \mathrm{H}), 7.26-7.44(\mathrm{~m}, 12 \mathrm{H}), 6.51-$ $6.55(\mathrm{~m}, 1 \mathrm{H}), 4.20(\mathrm{~d}, 2 \mathrm{H}, J=5 \mathrm{~Hz}), 3.79(\mathrm{~s}, 3 \mathrm{H}) .{ }^{13} \mathrm{C} \mathrm{NMR}\left(\mathrm{CDCl}_{3}, 75.46 \mathrm{MHz}\right) \delta / \mathrm{ppm}:$ $41.9(\mathrm{C} \alpha), 52.6(\mathrm{OMe}), 127.7(\mathrm{C} 4), 128.8\left(\mathrm{~d},{ }^{3} J_{\mathrm{CP}}=7 \mathrm{~Hz}, \mathrm{C} 3{ }^{\prime}\right), 129.0\left(\mathrm{~d},{ }^{2} J_{\mathrm{CP}}=5.5 \mathrm{~Hz}, \mathrm{C} 5\right)$, $129.2\left(\mathrm{C} 4\right.$ '), $132.3\left(\mathrm{~d},{ }^{2} J_{\mathrm{CP}}=24 \mathrm{~Hz}, \mathrm{C} 2\right), 133.9$ (d, ${ }^{2} J_{\mathrm{CP}}=20 \mathrm{~Hz}, \mathrm{C} 2$ '), 134.0 (C3, overlapped with C2' peak), $136.5\left(\mathrm{~d},{ }^{1} J_{\mathrm{CP}}=11 \mathrm{~Hz}, \mathrm{C} 1{ }^{\prime}\right), 136.9\left(\mathrm{~d},{ }^{2} J_{\mathrm{CP}}=16 \mathrm{~Hz}, \mathrm{C} 6\right), 138.7\left(\mathrm{~d},{ }^{1} J_{\mathrm{CP}}=13.5\right.$ $\mathrm{Hz}, \mathrm{C} 1), 167.3(\mathrm{C}(\mathrm{O}) \mathrm{NH}), 170.5$ (COOMe). ${ }^{31} \mathrm{P} \mathrm{NMR}\left(\mathrm{CDCl}_{3}, 242.93 \mathrm{MHz}\right) \delta / \mathrm{ppm}:-4.81$ (s, $1 \mathrm{P})$.

$\mathbf{P h}_{2} \mathbf{P}-\boldsymbol{m C}_{6} \mathbf{H}_{4}$-CO-Ala-OMe, $\mathbf{L}_{\mathbf{p A}}$; (Kokan et al 2014). 3-(Diphenylphosphino)benzoic acid (304.1 mg, $1 \mathrm{mmol})$, dichloromethane $(50 \mathrm{~mL}), \mathrm{HOBt} \times \mathrm{H}_{2} \mathrm{O}(136.2 \mathrm{mg}, 1 \mathrm{mmol})$, TBTU (321.7 mg, $1 \mathrm{mmol})$, DIPEA (0.5 mL, $4 \mathrm{mmol}), \mathrm{H}-\mathrm{Ala}-\mathrm{OMe} \times \mathrm{HCl}(142.3 \mathrm{mg}, 1 \mathrm{mmol}), 24$ h. Yield: $300.1 \mathrm{mg}(77 \%) .{ }^{1} \mathrm{H}$ NMR $\left(300.13 \mathrm{MHz}, \mathrm{CDCl}_{3}\right) \delta / \mathrm{ppm}: 7.76-7.80(\mathrm{~m}, 2 \mathrm{H}), 7.26-$ $7.44(\mathrm{~m}, 12 \mathrm{H}), 6.61(\mathrm{~d}, 1 \mathrm{H}, J=8), 4.70-4.80(\mathrm{~m}, 1 \mathrm{H}), 3.77(\mathrm{~s}, 1 \mathrm{H}), 1.48(\mathrm{~d}, 3 \mathrm{H}, \mathrm{J}=7 \mathrm{~Hz})$.

$\mathbf{P h}_{2} \mathbf{P}-\mathbf{C}_{2} \mathbf{H}_{4}$-CO-Gly-OMe, $\mathbf{L}_{\mathbf{a G}}$. 3-(Diphenylphosphino)propionic acid (262.3 $\mathrm{mg}, 1 \mathrm{mmol}$ ), dichloromethane $(50 \mathrm{~mL}), \mathrm{HOBt} \times \mathrm{H}_{2} \mathrm{O}(136.4 \mathrm{mg}, 1 \mathrm{mmol})$, TBTU $(324.3 \mathrm{mg}, 1 \mathrm{mmol})$, DIPEA (0.5 mL, $4 \mathrm{mmol})$, H-Gly-OMe $\times \mathrm{HCl}(127.2 \mathrm{mg}, 1 \mathrm{mmol}), 24 \mathrm{~h}$; Rf (Hexane/EtOAc $=7: 3)=0.10$. Yield: $310.2 \mathrm{mg}(94 \%) .{ }^{1} \mathrm{H}$ NMR $\left(300.13 \mathrm{MHz}, \mathrm{CDCl}_{3}\right) \delta / \mathrm{ppm}: 2.26-2.43(\mathrm{~m}$, 4H), 3.75 (s, 3H), 4.01 (d, $J=5 \mathrm{~Hz}, 2 \mathrm{H}), 5.93(\mathrm{t}, J=4 \mathrm{~Hz}, 1 \mathrm{H}), 7.31-7.36(\mathrm{~m}, 6 \mathrm{H}), 7.39$ $7.46(\mathrm{~m}, 4 \mathrm{H}) .{ }^{13} \mathrm{C} \mathrm{NMR}\left(\mathrm{CDCl}_{3}, 75.46 \mathrm{MHz}\right) \delta / \mathrm{ppm}: 23.4\left(\mathrm{~d},{ }^{1} J_{\mathrm{CP}}=12 \mathrm{~Hz}, \mathrm{C} 1\right.$ phosphine$)$, $32.6\left(\mathrm{~d},{ }^{2} J_{\mathrm{CP}}=18.5 \mathrm{~Hz}, \mathrm{C} 2\right.$ phosphine), $41.4(\mathrm{C \alpha}), 52.5(\mathrm{OMe}), 128.7\left(\mathrm{~d},{ }^{3} J_{\mathrm{CP}}=7 \mathrm{~Hz}, \mathrm{C} 3{ }^{\prime}\right.$ phosphine), 129.0 (C4 phosphine), $132.9\left(\mathrm{~d},{ }^{2} J_{\mathrm{CP}}=18.5 \mathrm{~Hz}, \mathrm{C} 2\right.$ ' phosphine), $137.9\left(\mathrm{~d},{ }^{1} J_{\mathrm{CP}}=\right.$ $12.5 \mathrm{~Hz}, \mathrm{C} 1$ ' phosphine), $170.6(\mathrm{COOMe}), 172.5\left(\mathrm{~d},{ }^{3} J_{\mathrm{CP}}=13.5 \mathrm{~Hz}, \mathrm{C}(\mathrm{O}) \mathrm{NH}\right)$.

$\mathbf{P h}_{\mathbf{2}} \mathbf{P}-\mathbf{C}_{2} \mathbf{H}_{4}$-CO-Ala-OMe, $\mathbf{L}_{\mathbf{a A}}$. 3-(Diphenylphosphino)propionic acid (261.9 $\mathrm{mg}, 1 \mathrm{mmol}$ ), dichloromethane $(50 \mathrm{~mL}), \mathrm{HOBt} \times \mathrm{H}_{2} \mathrm{O}(136.9 \mathrm{mg}, 1 \mathrm{mmol})$, TBTU $(327.3 \mathrm{mg}, 1 \mathrm{mmol})$, DIPEA (0.5 mL, $4 \mathrm{mmol}), \mathrm{H}-\mathrm{Ala}-\mathrm{OMe} \times \mathrm{HCl}(145.3 \mathrm{mg}, 1 \mathrm{mmol}), 24 \mathrm{~h}$. Rf (Hexane/EtOAc $=7: 3)=0.13$. Yield: $273.7 \mathrm{mg}(80 \%) .{ }^{1} \mathrm{H}$ NMR $\left(300.13 \mathrm{MHz}, \mathrm{CDCl}_{3}\right) \delta / \mathrm{ppm}: 1.37(\mathrm{~d}, J=7$ $\mathrm{Hz}, 3 \mathrm{H}), 2.23-2.41(\mathrm{~m}, 4 \mathrm{H}), 3.74(\mathrm{~s}, 3 \mathrm{H}), 4.52-4.61(\mathrm{~m}, 1 \mathrm{H}), 5.97(\mathrm{~d}, J=7 \mathrm{~Hz}, 1 \mathrm{H}), 7.31-$ $7.36(\mathrm{~m}, 6 \mathrm{H}), 7.39-7.46(\mathrm{~m}, 4 \mathrm{H}) .{ }^{13} \mathrm{C} \mathrm{NMR}\left(\mathrm{CDCl}_{3}, 75.46 \mathrm{MHz}\right) \delta / \mathrm{ppm}: 18.7(\mathrm{C} \beta), 23.4(\mathrm{~d}$, ${ }^{1} J_{\mathrm{CP}}=12 \mathrm{~Hz}, \mathrm{C} 1$ phosphine), $32.7\left(\mathrm{~d},{ }^{2} J_{\mathrm{CP}}=18.5 \mathrm{~Hz}, \mathrm{C} 2\right.$ phosphine), $48.2(\mathrm{C \alpha}), 52.6(\mathrm{OMe})$, $128.7\left(\mathrm{~d},{ }^{3} J_{\mathrm{CP}}=7 \mathrm{~Hz}, \mathrm{C} 3\right.$ ' phosphine), 128.93, 128.95 (C4 phosphine), 132.86, $132.89\left(\mathrm{~d},{ }^{2} J_{\mathrm{CP}}\right.$ $=18.5 \mathrm{~Hz}, \mathrm{C} 2$ ' phosphine), $137.89,137.96\left(\mathrm{~d},{ }^{1} J_{\mathrm{CP}}=13 \mathrm{~Hz}, \mathrm{C} 1\right.$ ' phosphine $), 171.8\left(\mathrm{~d},{ }^{3} J_{\mathrm{CP}}=\right.$ $14 \mathrm{~Hz}, \mathrm{C}(\mathrm{O}) \mathrm{NH}), 173.7$ (COOMe). ${ }^{31} \mathrm{P} \mathrm{NMR}\left(\mathrm{CDCl}_{3}, 242.93 \mathrm{MHz}\right) \delta / \mathrm{ppm}:-14.94$ (s, 1P).

ML complexes, general procedure. Ligand $\mathbf{L}$ was dissolved in DCM (3 mL), di- $\mu$ chlorobis[( $p$-cymene)chlororuthenium(II)] was added and stirred for $2 \mathrm{~h}$. After the reaction, the crude product was purified by column chromatography on a short silica column $(10 \mathrm{~g})$, eluent $\mathrm{DCM} / \mathrm{MeOH}$.

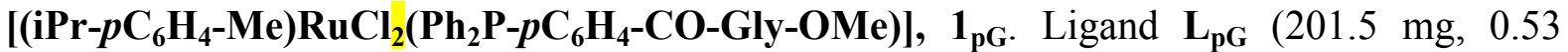
mmol) and di- $\mu$-chlorobis[( $p$-cymene)chlororuthenium(II)] (142.3, $0.23 \mathrm{mmol})$; eluent $\mathrm{DCM} / \mathrm{MeOH} 2 \%$, Rf (DCM/MeOH $3 \%)=0.28$. Yield: $279.9 \mathrm{mg}(89 \%) .{ }^{1} \mathrm{H}$ NMR (300.13 
$\left.\mathrm{MHz}, \mathrm{CDCl}_{3}\right) \delta / \mathrm{ppm}: 7.69-7.95(\mathrm{~m}, 8 \mathrm{H}), 7.37-7.47(\mathrm{~m}, 6 \mathrm{H}), 6.64(\mathrm{t}, 1 \mathrm{H}, J=5 \mathrm{~Hz}), 5.22(\mathrm{~d}$, $2 \mathrm{H}, J=6 \mathrm{~Hz}), 4.98(\mathrm{~d}, 2 \mathrm{H}, J=6 \mathrm{~Hz}), 4.21(\mathrm{~d}, 2 \mathrm{H}, J=5 \mathrm{~Hz}), 3.78(\mathrm{~s}, 3 \mathrm{H}), 3.49(\mathrm{~d}, 1 \mathrm{H}, J=$ $5.5 \mathrm{~Hz}), 2.81-2.91(\mathrm{~m}, 1 \mathrm{H}), 1.86(\mathrm{~s}, 3 \mathrm{H}), 1.12(\mathrm{~d}, 6 \mathrm{H}, \mathrm{J}=7 \mathrm{~Hz}) .{ }^{13} \mathrm{C}$ NMR $\left(\mathrm{CDCl}_{3}, 150.92\right.$ MHz) $\delta / \mathrm{ppm}: 18.0\left(\mathrm{CH}_{3}\right.$, cymene $), 22.0\left(\mathrm{CHCH}_{3}\right.$, cymene), $30.4\left(\mathrm{CHCH}_{3}\right.$, cymene $), 41.9$ $(\mathrm{C} \alpha), 52.6(\mathrm{OMe}), 87.5\left(\mathrm{~d},{ }^{2} J_{\mathrm{CP}}=5.5 \mathrm{~Hz}, \mathrm{C} 2, \mathrm{C} 6\right.$, cymene), $89.0\left(\mathrm{~d},{ }^{2} J_{\mathrm{CP}}=3 \mathrm{~Hz}, \mathrm{C} 3, \mathrm{C} 5\right.$, cymene), 96.4 (C4, cymene), $111.6\left(\mathrm{~d},{ }^{2} J_{\mathrm{CP}}=3.5 \mathrm{~Hz}, \mathrm{C} 1\right.$, cymene), $126.5\left(\mathrm{~d},{ }^{3} J_{\mathrm{CP}}=10 \mathrm{~Hz}\right.$, $\mathrm{C} 3$, phosphine), $128.4\left(\mathrm{~d},{ }^{3} J_{\mathrm{CP}}=10 \mathrm{~Hz}, \mathrm{C} 3\right.$ ', phosphine), 130.7 (C4', phosphine), 133.5 (d, ${ }^{1} J_{\mathrm{CP}}=45 \mathrm{~Hz}, \mathrm{C} 1$ ', phosphine), $134.3\left(\mathrm{~d},{ }^{2} J_{\mathrm{CP}}=9.5 \mathrm{~Hz}, \mathrm{C} 2\right.$ ', phosphine), $134.8\left(\mathrm{~d},{ }^{2} J_{\mathrm{CP}}=9.5\right.$ $\mathrm{Hz}, \mathrm{C} 2$, phosphine), 135.1 (C4, phosphine), 137.7 (d, ${ }^{1} J_{\mathrm{CP}}=44 \mathrm{~Hz}, \mathrm{C} 1$, phosphine), 167.1 (C(O)NH), 170.4 (COOMe). ${ }^{31} \mathrm{P}$ NMR $\left(\mathrm{CDCl}_{3}, 242.93 \mathrm{MHz}\right) \delta / \mathrm{ppm}: 25.32$ (s, 1P). MALDIHRMS $(\mathrm{m} / \mathrm{z})$ : calcd $613.1320\left(\mathrm{C}_{32} \mathrm{H}_{34} \mathrm{NO}_{3} \mathrm{PRu}^{+}\right)$, found 613.1329 .

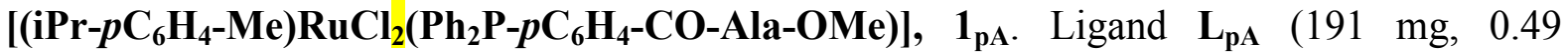
$\mathrm{mmol})$ and di- $\mu$-chlorobis[( $p$-cymene)chlororuthenium(II)] (131.2, $0.21 \mathrm{mmol})$; eluent $\mathrm{DCM} / \mathrm{MeOH} 2 \%$, Rf (DCM/MeOH $3 \%)=0.28$. Yield: $218.3 \mathrm{mg}(75 \%) .{ }^{1} \mathrm{H}$ NMR (300.13 $\left.\mathrm{MHz}, \mathrm{CDCl}_{3}\right) \delta / \mathrm{ppm}: 7.69-7.95(\mathrm{~m}, 8 \mathrm{H}), 7.37-7.47(\mathrm{~m}, 6 \mathrm{H}), 6.67(\mathrm{~d}, 1 \mathrm{H}, J=7.5 \mathrm{~Hz}), 5.21-$ $5.24(\mathrm{~m}, 2 \mathrm{H}), 4.98(\mathrm{~d}, 2 \mathrm{H}, J=6 \mathrm{~Hz}), 4.71-4.80(\mathrm{~m}, 1 \mathrm{H}), 3.77(\mathrm{~s}, 3 \mathrm{H}), 2.8-2.93(\mathrm{~m}, 1 \mathrm{H}), 1.87$ $(\mathrm{s}, 3 \mathrm{H}), 1.49(\mathrm{~d}, 3 \mathrm{H}, J=7 \mathrm{~Hz}), 1.12(\mathrm{~d}, 6 \mathrm{H}, J=7 \mathrm{~Hz}) \cdot{ }^{13} \mathrm{C} \mathrm{NMR}\left(\mathrm{CDCl}_{3}, 150.92 \mathrm{MHz}\right)$ $\delta /$ ppm: $18.0\left(\mathrm{CH}_{3}\right.$, cymene $), 18.6(\mathrm{C} \beta), 22.0\left(\mathrm{CHCH}_{3}\right.$, cymene $), 30.4\left(\mathrm{CHCH}_{3}\right.$, cymene $), 48.7$ $(\mathrm{C} \alpha), 52.7(\mathrm{OMe}), 87.48,87.52\left(\mathrm{~d},{ }^{2} J_{\mathrm{CP}}=5 \mathrm{~Hz}, \mathrm{C} 2, \mathrm{C} 6\right.$, cymene), 88.99, $89.04\left(\mathrm{~d},{ }^{2} J_{\mathrm{CP}}=3\right.$ $\mathrm{Hz}, \mathrm{C} 3, \mathrm{C} 5$, cymene), 96.3 (C4, cymene), $111.6\left(\mathrm{~d},{ }^{2} J_{\mathrm{CP}}=3.5 \mathrm{~Hz}, \mathrm{C} 1\right.$, cymene), 126.4 (d, ${ }^{3} J_{\mathrm{CP}}$ $=10 \mathrm{~Hz}, \mathrm{C} 3$, phosphine), $128.3\left(\mathrm{~d},{ }^{3} J_{\mathrm{CP}}=10 \mathrm{~Hz}, \mathrm{C} 3\right.$ ', phosphine), 130.7 (C4', phosphine), 133.51, $133.52\left(\mathrm{~d},{ }^{1} J_{\mathrm{CP}}=45 \mathrm{~Hz}, \mathrm{C} 1\right.$ ', phosphine), $134.4\left(\mathrm{~d},{ }^{2} J_{\mathrm{CP}}=9.5 \mathrm{~Hz}, \mathrm{C} 2\right.$ ', phosphine), $134.8\left(\mathrm{~d},{ }^{2} J_{\mathrm{CP}}=9.5 \mathrm{~Hz}, \mathrm{C} 2\right.$, phosphine), 135.28, 135.29 (C4, phosphine), $137.7\left(\mathrm{~d},{ }^{1} J_{\mathrm{CP}}=\right.$ $44.5 \mathrm{~Hz}, \mathrm{Cl}$, phosphine), $166.5(\mathrm{C}(\mathrm{O}) \mathrm{NH}), 173.4$ (COOMe). ${ }^{31} \mathrm{P} \mathrm{NMR}\left(\mathrm{CDCl}_{3}, 242.93 \mathrm{MHz}\right)$ $\delta / \mathrm{ppm}$ : $25.31(\mathrm{~s}, 1 \mathrm{P})$. MALDI-HRMS $(\mathrm{m} / \mathrm{z})$ : calcd $662.1165\left(\mathrm{C}_{33} \mathrm{H}_{36} \mathrm{ClNO}_{3} \mathrm{PRu}^{+}\right)$, found 662.1131 .

[(iPr-pC $\left.\left.\mathbf{C}_{6} \mathbf{H}_{4}-\mathrm{Me}\right) \mathrm{RuCl}_{\mathbf{2}}\left(\mathbf{P h}_{2} \mathbf{P}-\mathbf{m C}_{\mathbf{6}} \mathbf{H}_{4}-\mathbf{C O}-\mathbf{G l y}-\mathrm{OMe}\right)\right], \mathbf{1}_{\mathrm{mG}}$. Ligand $\mathbf{L}_{\mathbf{m G}}(182.2 \mathrm{mg}, 0.48$ mmol) and di- $\mu$-chlorobis[( $p$-cymene)chlororuthenium(II)] (147.1, $0.24 \mathrm{mmol})$; eluent $\mathrm{DCM} / \mathrm{MeOH} 2 \%$, Rf (DCM/MeOH $3 \%)=0.26$. Yield: $301.1 \mathrm{mg}(92 \%) .{ }^{1} \mathrm{H}$ NMR (300.13 $\left.\mathrm{MHz}, \mathrm{CDCl}_{3}\right) \delta / \mathrm{ppm}: 8.83\left(\mathrm{dt}, 1 \mathrm{H}, J_{1}=12 \mathrm{hz}, J_{2}=1.5 \mathrm{~Hz}\right), 7.78-7.91(\mathrm{~m}, 5 \mathrm{H}), 7.56-7.63$ $(\mathrm{m}, 1 \mathrm{H}), 7.31-7.49(\mathrm{~m}, 7 \mathrm{H}), 6.93(\mathrm{t}, 1 \mathrm{H}, J=5.5 \mathrm{~Hz}), 5.22(\mathrm{~d}, 2 \mathrm{H}, J=6 \mathrm{~Hz}), 5.07(\mathrm{~d}, 2 \mathrm{H}, J=$ $6 \mathrm{~Hz}), 5.19(\mathrm{~d}, 2 \mathrm{H}, J=5.5 \mathrm{~Hz}), 3.77(\mathrm{~s}, 3 \mathrm{H}), 2.77-2.86(\mathrm{~m}, 1 \mathrm{H}), 1.88(\mathrm{~s}, 3 \mathrm{H}), 1.08(\mathrm{~d}, 6 \mathrm{H}, J=$ $7 \mathrm{~Hz}) .{ }^{13} \mathrm{C} \mathrm{NMR}\left(\mathrm{CDCl}_{3}, 150.92 \mathrm{MHz}\right) \delta / \mathrm{ppm}: 17.9\left(\mathrm{CH}_{3}\right.$, cymene $), 21.9\left(\mathrm{CHCH}_{3}\right.$, cymene $)$, $30.4\left(\mathrm{CHCH}_{3}\right.$, cymene), $41.9(\mathrm{C \alpha}), 52.4(\mathrm{OMe}), 87.2\left(\mathrm{~d},{ }^{2} J_{\mathrm{CP}}=5.5 \mathrm{~Hz}, \mathrm{C} 2, \mathrm{C} 6\right.$, cymene $)$, $89.5\left(\mathrm{~d},{ }^{2} J_{\mathrm{CP}}=2.5 \mathrm{~Hz}, \mathrm{C} 3, \mathrm{C} 5\right.$, cymene), 96.2 (C4, cymene), $111.6\left(\mathrm{~d},{ }^{2} J_{\mathrm{CP}}=2.5 \mathrm{~Hz}, \mathrm{C} 1\right.$, cymene), 128.3 (d, ${ }^{3} J_{\mathrm{CP}}=8.5 \mathrm{~Hz}, \mathrm{C} 5$, phosphine), 128.5 (d, ${ }^{3} J_{\mathrm{CP}}=9.5 \mathrm{~Hz}, \mathrm{C} 3$ ', phosphine), 129.8 (C4, phosphine), 130.8 (C4', phosphine), 132.4 (d, ${ }^{1} J_{\mathrm{CP}}=46 \mathrm{~Hz}, \mathrm{C} 1$ ', phosphine), 132.9 $\left(\mathrm{d},{ }^{3} J_{\mathrm{CP}}=11 \mathrm{~Hz}, \mathrm{C} 3\right.$, phosphine), $133.9\left(\mathrm{~d},{ }^{2} J_{\mathrm{CP}}=9.5 \mathrm{~Hz}, \mathrm{C} 2\right.$ ', phosphine $), 134.1\left(\mathrm{~d},{ }^{1} J_{\mathrm{CP}}=46\right.$ $\mathrm{Hz}, \mathrm{C} 1$, phosphine), 135.2 (d, ${ }^{2} J_{\mathrm{CP}}=16 \mathrm{~Hz}, \mathrm{C} 2$, phosphine), 136.73, 136.75 (C6, phosphine), $167.2(\mathrm{C}(\mathrm{O}) \mathrm{NH}), 170.3(\mathrm{COOMe}) .{ }^{31} \mathrm{P} \mathrm{NMR}\left(\mathrm{CDCl}_{3}, 242.93 \mathrm{MHz}\right) \delta / \mathrm{ppm}: 25.50(\mathrm{~s}, 1 \mathrm{P})$. + ESI MS $(\mathrm{m} / \mathrm{z}): 648.1\left([\mathrm{M}-\mathrm{Cl}]^{+}, 81 \%\right)$. MALDI-HRMS $(\mathrm{m} / \mathrm{z})$ : calcd 612.1241 $\left(\mathrm{C}_{32} \mathrm{H}_{33} \mathrm{NO}_{3} \mathrm{PRu}^{+}\right)$, found 612.1226 .

[(iPr-pC $\left.\left.\mathrm{C}_{6} \mathrm{H}_{4}-\mathrm{Me}\right) \mathrm{RuCl}_{2}\left(\mathrm{Ph}_{2} \mathbf{P}-\mathrm{mC}_{6} \mathrm{H}_{4}-\mathrm{CO}-\mathrm{Ala}-\mathrm{OMe}\right)\right], \mathbf{1}_{\mathrm{mA}}$. Ligand $\mathrm{L}_{\mathrm{mA}}(180 \mathrm{mg}, 0.46$ $\mathrm{mmol})$ and di- $\mu$-chlorobis[( $p$-cymene)chlororuthenium(II)] (141.6, $0.23 \mathrm{mmol})$; eluent $\mathrm{DCM} / \mathrm{MeOH} 2 \%$, Rf (DCM/MeOH $3 \%)=0.28$. Yield: $302 \mathrm{mg}(94 \%)$. ${ }^{1} \mathrm{H}$ NMR (300.13 $\left.\mathrm{MHz}, \mathrm{CDCl}_{3}\right) \delta / \mathrm{ppm}: 8.75-8.80(\mathrm{~m}, 1 \mathrm{H}), 7.76-7.78(\mathrm{~m}, 5 \mathrm{H}), 7.61-7.68(\mathrm{~m}, 1 \mathrm{H}), 7.31-7.47$ $(\mathrm{m}, 7 \mathrm{H}), 6.88(\mathrm{~d}, 1 \mathrm{H}, \mathrm{J}=8 \mathrm{H}), 5.26-5.30(\mathrm{~m}, 2 \mathrm{H}), 5.17(\mathrm{~d}, 2 \mathrm{H}, J=6.5 \mathrm{~Hz}), 4.96(\mathrm{~d}, 2 \mathrm{H}, \mathrm{J}=6$ 
$\mathrm{Hz}), 4.65-4.75(\mathrm{~m}, 1 \mathrm{H}), 3.76(\mathrm{~s}, 3 \mathrm{H}), 2.75-2.89(\mathrm{~m}, 1 \mathrm{H}), 1.88(\mathrm{~s}, 3 \mathrm{H}), 1.52(\mathrm{~d}, 3 \mathrm{H}, J=7 \mathrm{~Hz})$, 1.08 (t, $6 \mathrm{H}, J=7.5 \mathrm{~Hz}) .{ }^{13} \mathrm{C} \mathrm{NMR}\left(\mathrm{CDCl}_{3}, 150.92 \mathrm{MHz}\right) \delta / \mathrm{ppm}: 17.9\left(\mathrm{CH}_{3}\right.$, cymene $), 18.3$ $(\mathrm{C} \beta), 21.8,22.0\left(\mathrm{CHCH}_{3}\right.$, cymene $), 30.4\left(\mathrm{CHCH}_{3}\right.$, cymene $), 48.8(\mathrm{C \alpha}), 52.6(\mathrm{OMe}), 86.987 .6$ $\left(\mathrm{d},{ }^{2} J_{\mathrm{CP}}=5.5 \mathrm{~Hz}, \mathrm{C} 2, \mathrm{C} 6\right.$, cymene), $89.3,89.4$ (d, ${ }^{2} J_{\mathrm{CP}}=2.5 \mathrm{~Hz}, \mathrm{C} 3, \mathrm{C} 5$, cymene), 96.3 (C4, cymene), $111.5\left(\mathrm{~d},{ }^{2} J_{\mathrm{CP}}=3 \mathrm{~Hz}, \mathrm{C} 1\right.$, cymene), $128.3\left(\mathrm{~d},{ }^{3} J_{\mathrm{CP}}=8.5 \mathrm{~Hz}, \mathrm{C} 5\right.$, phosphine), 128.5 (d, ${ }^{3} J_{\mathrm{CP}}=9.5 \mathrm{~Hz}, \mathrm{C} 3$ ', phosphine), $129.6(\mathrm{C} 4$, phosphine),130.8 (C4', phosphine), $132.6(\mathrm{~d}$, ${ }^{1} J_{\mathrm{CP}}=46 \mathrm{~Hz}, \mathrm{C} 1$ ', phosphine), 133.1 (d, ${ }^{3} J_{\mathrm{CP}}=11 \mathrm{~Hz}, \mathrm{C} 3$, phosphine), 133.9-134.4 (C2', C1, phosphine), 135.1 (d, ${ }^{2} J_{\mathrm{CP}}=16 \mathrm{~Hz}, \mathrm{C} 2$, phosphine), 136.72, 136.74 (C6, phosphine), 166.5 $(\mathrm{C}(\mathrm{O}) \mathrm{NH}), 173.4$ (COOMe). ${ }^{31} \mathrm{P}$ NMR $\left(\mathrm{CDCl}_{3}, 242.93 \mathrm{MHz}\right) \delta / \mathrm{ppm}: 25.49$ (s, 1P). +ESI MS $(\mathrm{m} / \mathrm{z}): 662.1\left([\mathrm{M}-\mathrm{Cl}]^{+}, 100 \%\right)$. MALDI-HRMS $(\mathrm{m} / \mathrm{z})$ : calcd $627.1476\left(\mathrm{C}_{33} \mathrm{H}_{36} \mathrm{NO}_{3} \mathrm{PRu}^{+}\right)$, found 627.1504 .

[(iPr-pC $\left.\left.\mathbf{C}_{6} \mathbf{H}_{4}-\mathbf{M e}\right) \mathbf{R u C l}_{\mathbf{2}}\left(\mathbf{P h}_{\mathbf{2}} \mathbf{P}-\mathbf{C}_{2} \mathbf{H}_{4}-\mathbf{C O}-\mathbf{G l y}-\mathbf{O M e}\right)\right], \mathbf{1}_{\mathrm{aG}}$. Ligand $\mathbf{L}_{\mathrm{aG}}(180 \mathrm{mg}, 0.55 \mathrm{mmol})$ and di- $\mu$-chlorobis[( $p$-cymene)chlororuthenium(II)] (167.9, $0.28 \mathrm{mmol})$; eluent $\mathrm{DCM} / \mathrm{MeOH}$ $1 \%$ using $40 \mathrm{~g}$ silica, $\mathrm{Rf}(\mathrm{DCM} / \mathrm{MeOH} 3 \%)=0.34$. Yield: $297.2 \mathrm{mg}(85 \%)$. ${ }^{1} \mathrm{H}$ NMR $\left(300.13 \mathrm{MHz}, \mathrm{CDCl}_{3}\right) \delta / \mathrm{ppm}: 0.98(\mathrm{~d}, J=7 \mathrm{~Hz}, 6 \mathrm{H}), 2.21-2.26(\mathrm{~m}, 2 \mathrm{H}), 2.62$ (septuplet, $J=$ $7 \mathrm{~Hz}, 1 \mathrm{H}), 2.88-2.93(\mathrm{~m}, 2 \mathrm{H}), 3.68(\mathrm{~s}, 3 \mathrm{H}), 3.78(\mathrm{~d}, J=5.5 \mathrm{~Hz}, 2 \mathrm{H}), 5.09(\mathrm{~d}, J=6 \mathrm{~Hz}, 2 \mathrm{H})$, $5.18(\mathrm{~d}, J=6 \mathrm{~Hz}, 2 \mathrm{H}), 6.21(\mathrm{t}, J=5 \mathrm{~Hz}, 2 \mathrm{H}), 7.45-7.50(\mathrm{~m}, 6 \mathrm{H}), 7.78-7.80(\mathrm{~m}, 4 \mathrm{H}) .{ }^{13} \mathrm{C}$ NMR $\left(\mathrm{CDCl}_{3}, 75.46 \mathrm{MHz}\right) \delta / \mathrm{ppm}: 17.7\left(\mathrm{CH}_{3}\right.$, cymene $), 21.8\left(\mathrm{CHCH}_{3}\right.$, cymene $), 22.9\left(\mathrm{~d},{ }^{1} J_{\mathrm{CP}}\right.$ $=30 \mathrm{~Hz}, \mathrm{C} 1$ phosphine), $30.2\left(\mathrm{CHCH}_{3}\right.$, cymene $), 30.9\left(\mathrm{~d},{ }^{2} J_{\mathrm{CP}}=2 \mathrm{~Hz}, \mathrm{C} 2\right.$ phosphine $), 41.3$ $(\mathrm{C} \alpha), 52.3(\mathrm{OMe}), 86.1\left(\mathrm{~d},{ }^{2} J_{\mathrm{CP}}=6 \mathrm{~Hz}, \mathrm{C} 2, \mathrm{C} 6\right.$, cymene), $90.1\left(\mathrm{~d},{ }^{2} J_{\mathrm{CP}}=4 \mathrm{~Hz}, \mathrm{C} 3, \mathrm{C} 5\right.$, cymene), 95.1 (C4, cymene), $109.5\left(\mathrm{~d},{ }^{2} J_{\mathrm{CP}}=1 \mathrm{~Hz}, \mathrm{C} 1\right.$, cymene), $128.6\left(\mathrm{~d},{ }^{3} J_{\mathrm{CP}}=9 \mathrm{~Hz}, \mathrm{C} 3^{\prime}\right.$ phosphine), 130.90, 130.93 (C4' phosphine), 133.3 (d, ${ }^{2} J_{\mathrm{CP}}=9 \mathrm{~Hz}, \mathrm{C} 2$ ' phosphine), 133.5 (d, ${ }^{1} J_{\mathrm{CP}}=43 \mathrm{~Hz}, \mathrm{C} 1$ ' phosphine), $170.2(\mathrm{COOMe}), 172.6\left(\mathrm{~d},{ }^{3} J_{\mathrm{CP}}=13 \mathrm{~Hz}, \mathrm{C}(\mathrm{O}) \mathrm{NH}\right) .{ }^{31} \mathrm{P}$ NMR $\left(\mathrm{CDCl}_{3}, 242.93 \mathrm{MHz}\right) \delta / \mathrm{ppm}: 21.36$ (s, 1P). MALDI-HRMS (m/z): calcd 600.1008 $\left(\mathrm{C}_{28} \mathrm{H}_{34} \mathrm{ClNO}_{3} \mathrm{PRu}^{+}\right)$, found 600.1011 .

[(iPr-pC $\left.\left.\mathbf{C}_{6} \mathbf{H}_{4}-\mathbf{M e}\right) \mathbf{R u C l}_{2}\left(\mathbf{P h}_{2} \mathbf{P}-\mathbf{C}_{2} \mathbf{H}_{4}-\mathbf{C O}-A l a-O M e\right)\right], \mathbf{1}_{\mathrm{aA}}$. Ligand $\mathbf{L}_{\mathbf{a A}}(164 \mathrm{mg}, 0.48 \mathrm{mmol})$ and di- $\mu$-chlorobis[( $p$-cymene)chlororuthenium(II)] (146.3, 0.24mmol); eluent DCM/MeOH $1 \%$ using 40 g. Yield: $196.8 \mathrm{mg}(63 \%) .{ }^{1} \mathrm{H}$ NMR (300.13 MHz, $\left.\mathrm{CDCl}_{3}\right) \delta / \mathrm{ppm}$ : $0.96(\mathrm{~d}, J=$ $7 \mathrm{~Hz}, 3 \mathrm{H}), 0.98(\mathrm{~d}, J=7 \mathrm{~Hz}, 3 \mathrm{H}), 1.28(\mathrm{~d}, J=7 \mathrm{~Hz}, 3 \mathrm{H}), 2.06-2.29$ (m, 2H), 2.63 (septuplet, $J=7 \mathrm{~Hz}, 1 \mathrm{H}), 2.85-2.94(\mathrm{~m}, 2 \mathrm{H}), 3.67(\mathrm{~s}, 3 \mathrm{H}), 4.26-4.36(\mathrm{~m}, 1 \mathrm{H}), 5.07-5.11(\mathrm{~m}, 2 \mathrm{H}), 5.17-$ $5.20(\mathrm{~m}, 2 \mathrm{H}), 6.14(\mathrm{~d}, J=7 \mathrm{~Hz}, 1 \mathrm{H}), 7.43-7.50(\mathrm{~m}, 6 \mathrm{H}), 7.76-7.82(\mathrm{~m}, 4 \mathrm{H}) .{ }^{13} \mathrm{C}$ NMR $\left(\mathrm{CDCl}_{3}, 75.46 \mathrm{MHz}\right) \delta / \mathrm{ppm}: 17.7\left(\mathrm{CH}_{3}\right.$, cymene $), 18.2(\mathrm{C} \beta), 21.7,21.9\left(\mathrm{CHCH}_{3}\right.$, cymene $)$, $22.9\left(\mathrm{~d},{ }^{1} J_{\mathrm{CP}}=31 \mathrm{~Hz}, \mathrm{C} 1\right.$ phosphine $), 30.2\left(\mathrm{CHCH}_{3}\right.$, cymene), 31.0 (C2 phosphine), 48.2 $(\mathrm{C} \alpha), 52.4(\mathrm{OMe}), 86.1\left(\mathrm{~d},{ }^{2} J_{\mathrm{CP}}=5.5 \mathrm{~Hz}, \mathrm{C} 2, \mathrm{C} 6\right.$, cymene), $90.1\left(\mathrm{~d},{ }^{2} J_{\mathrm{CP}}=4 \mathrm{~Hz}, \mathrm{C} 3, \mathrm{C} 5\right.$, cymene), 95.2 (C4, cymene), $109.5\left(\mathrm{~d},{ }^{2} J_{\mathrm{CP}}=1 \mathrm{~Hz}, \mathrm{C} 1\right.$, cymene), $128.59,128.62\left(\mathrm{~d},{ }^{3} J_{\mathrm{CP}}=\right.$ $9.5 \mathrm{~Hz}, \mathrm{C} 3$ ' phosphine), 130.88-130.92 (m, C4' phosphine), 133.3, 133.4 (d, ${ }^{2} J_{\mathrm{CP}}=8 \mathrm{~Hz}, \mathrm{C} 2$ ' phosphine), 133.56, $133.62\left(\mathrm{~d},{ }^{1} J_{\mathrm{CP}}=43 \mathrm{~Hz}, \mathrm{Cl}\right.$ ' phosphine), $172.0\left(\mathrm{~d},{ }^{3} J_{\mathrm{CP}}=14 \mathrm{~Hz}\right.$, $\mathrm{C}(\mathrm{O}) \mathrm{NH}), 173.3$ (COOMe). ${ }^{31} \mathrm{P} \mathrm{NMR}\left(\mathrm{CDCl}_{3}, 242.93 \mathrm{MHz}\right) \delta / \mathrm{ppm}: 21.35$ (s, 1P). MALDIHRMS $(\mathrm{m} / z)$ : calcd $614.1165\left(\mathrm{C}_{29} \mathrm{H}_{36} \mathrm{ClNO}_{3} \mathrm{PRu}^{+}\right)$, found 614.1180 .

$\mathrm{ML}_{2}$ complexes, general procedure. Mono complex 1 and $\mathrm{NH}_{4} \mathrm{PF}_{6}$ were dissolved in $\mathrm{CH}_{3} \mathrm{CN}(5 \mathrm{~mL})$ and refluxed for $35-45 \mathrm{~min}$. $\mathrm{CH}_{3} \mathrm{CN}$ was evaporated, the residue dissolved in DCM (5 mL) and filtrated through Celite. Ligand $\mathbf{L}$ was dissolved in DCM (2 mL), added to the filtrate and the mixture was stirred for $24 \mathrm{~h}$. The crude product was purified by column chromatography on silica (40g), using DCM/MeOH $1 \%$ as eluent.

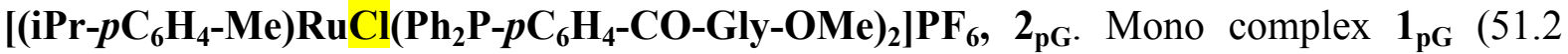
$\mathrm{mg}, 0.075 \mathrm{mmol}), \mathrm{NH}_{4} \mathrm{PF}_{6}(18.2 \mathrm{mg}, 0.11 \mathrm{mmol})$ and ligand $\mathbf{L}_{\mathbf{p G}}(70 \mathrm{mg}, 0.19 \mathrm{mmol}) . \mathrm{Rf}$ 
(DCM/MeOH $3 \%$ ) 0.18. Yield: $32.6 \mathrm{mg}(37 \%) .{ }^{1} \mathrm{H}$ NMR (300.13 MHz, $\left.\mathrm{CDCl}_{3}\right) \delta / \mathrm{ppm}$ : $7.23-7.60(\mathrm{~m}, 28 \mathrm{H}), 7.12(\mathrm{t}, 2 \mathrm{H}, J=5.5 \mathrm{~Hz}), 5.53(\mathrm{~d}, 2 \mathrm{H}, J=4 \mathrm{~Hz}), 5.14(\mathrm{~d}, 2 \mathrm{H}, J=6 \mathrm{H})$, $4.21(\mathrm{~d}, 4 \mathrm{H}, J=5.5 \mathrm{~Hz}), 3.78(\mathrm{~s}, 6 \mathrm{H}), 2.68-2.77(\mathrm{~m}, 1 \mathrm{H}), 1.24(\mathrm{~d}, 6 \mathrm{H}, J=7 \mathrm{~Hz}), 1.09(\mathrm{~s}, 3 \mathrm{H})$. ${ }^{13} \mathrm{C}$ NMR $\left(\mathrm{CDCl}_{3}, 150.92 \mathrm{MHz}\right) \delta / \mathrm{ppm}$ : $15.5\left(\mathrm{CH}_{3}\right.$, cymene), $21.6\left(\mathrm{CHCH}_{3}\right.$, cymene $), 31.6$ $\left(\mathrm{CHCH}_{3}\right.$, cymene), $41.9(\mathrm{C \alpha}), 52.5(\mathrm{OMe}), 89.3$ (t, ${ }^{2} J_{\mathrm{CP}}=5 \mathrm{~Hz}, \mathrm{C} 2, \mathrm{C} 6$, cymene), 97.6 (C3, C5, cymene), 101.0 (C4, cymene), 126.97, 127.01 (d, ${ }^{3} J_{\mathrm{CP}}=5 \mathrm{~Hz}, \mathrm{C} 3$, phosphine), 128.64, 128.68, 128.85, $128.88\left(\mathrm{~d},{ }^{3} J_{\mathrm{CP}}=5 \mathrm{~Hz}, \mathrm{C} 3\right.$ ', phosphine), 131.2, 131.6 (C4', phosphine), 131.8 (C1, cymene), 133.2-133.9 (m, C1' phosphine), 134.12, 134.16, 134.26, 134.30, 134.49, $134.52\left(\mathrm{~d},{ }^{2} J_{\mathrm{CP}}=4 \mathrm{~Hz}, \mathrm{C} 2\right.$, C2', phosphine), 136.2 (C4, phosphine), $136.4\left(\mathrm{~d},{ }^{2} J_{\mathrm{CP}}=45 \mathrm{~Hz}\right.$, $\mathrm{C} 1$, phosphine), $167.2(\mathrm{C}(\mathrm{O}) \mathrm{NH}), 170.3(\mathrm{COOMe}) .{ }^{31} \mathrm{P} \mathrm{NMR}\left(\mathrm{CDCl}_{3}, 242.93 \mathrm{MHz}\right) \delta / \mathrm{ppm}:-$ 143.93 (septuplet, hexafluorophosphate), 21.42 (s, 1P). MALDI-HRMS (m/z): calcd 991.2579 $\left(\mathrm{C}_{54} \mathrm{H}_{5455} \mathrm{~N}_{2} \mathrm{O}_{6} \mathrm{P}_{2} \mathrm{Ru}^{+}\right)$, found 991.2537.

[(iPr-pC $\left.\left.\mathrm{C}_{6} \mathrm{H}_{4}-\mathrm{Me}\right) \mathrm{RuCl}\left(\mathrm{Ph}_{2} \mathbf{P}-p \mathrm{C}_{6} \mathrm{H}_{\mathbf{4}}-\mathrm{CO}-\mathrm{Ala}-\mathrm{OMe}\right)_{2}\right] \mathbf{P F} \mathbf{6}_{6}, \mathbf{2}_{\mathrm{pA}}$. Mono complex $\mathbf{1}_{\mathrm{pA}}(70 \mathrm{mg}$, $0.1 \mathrm{mmol}), \mathrm{NH}_{4} \mathrm{PF}_{6}(24.5 \mathrm{mg}, 0.15 \mathrm{mmol})$ and ligand $\mathbf{L}_{\mathbf{p A}}(100 \mathrm{mg}, 0.25 \mathrm{mmol}) . \mathrm{Rf}$ (DCM/MeOH $3 \%)$ 0.22. Yield: $84.5 \mathrm{mg}(71 \%)$. ${ }^{1} \mathrm{H}$ NMR (300.13 $\left.\mathrm{MHz}, \mathrm{CDCl}_{3}\right) \delta / \mathrm{ppm}$ : $7.26-7.58(\mathrm{~m}, 28 \mathrm{H}), 7.03(\mathrm{~d}, 2 \mathrm{H}, \mathrm{J}=7 \mathrm{~Hz}), 5.55(\mathrm{t}, 2 \mathrm{H}, J=4.5 \mathrm{~Hz}), 5.24(\mathrm{~d}, 1 \mathrm{H}, J=6 \mathrm{~Hz})$, $5.09(\mathrm{~d}, 1 \mathrm{H}, J=6 \mathrm{~Hz}), 4.66-4.76(\mathrm{~m}, 2 \mathrm{H}), 3.78(\mathrm{~s}, 6 \mathrm{H}), 2.67-2.76(\mathrm{~m}, 1 \mathrm{H}), 1.54(\mathrm{~d}, 6 \mathrm{H}, \mathrm{J}=7$ $\mathrm{Hz}), 1.24(\mathrm{t}, 6 \mathrm{H}, J=7 \mathrm{~Hz}), 1.08(\mathrm{~s}, 3 \mathrm{H}) .{ }^{13} \mathrm{C} \mathrm{NMR}\left(\mathrm{CDCl}_{3}, 150.92 \mathrm{MHz}\right) \delta / \mathrm{ppm}: 15.4\left(\mathrm{CH}_{3}\right.$, cymene), $17.9(\mathrm{C} \beta), 21.54,21.58\left(\mathrm{CHCH}_{3}\right.$, cymene $), 31.6\left(\mathrm{CHCH}_{3}\right.$, cymene $), 49.0(\mathrm{C} \alpha), 52.7$ (OMe), 89.3, $89.6\left(\mathrm{~d},{ }^{2} J_{\mathrm{CP}}=9.5 \mathrm{~Hz}, \mathrm{C} 2, \mathrm{C} 6\right.$, cymene), 97.4, $97.7\left(\mathrm{~d},{ }^{3} J_{\mathrm{CP}}=3 \mathrm{~Hz}, \mathrm{C} 3, \mathrm{C} 5\right.$, cymene), 101.0 (C4, cymene), 126.9-127.0 (m, C3, phosphine), 128.6-128.8 (m, C3', phosphine), 131.19, 131.22, 131.47 (C4', phosphine), 131.8 (C1, cymene), 133.1-133.9 (m, C1', phosphine), 134.0-134.5 (m, C2, C2', phosphine), 136.3, 136.4 (C4, phosphine), 136.5 $\left(\mathrm{d},{ }^{2} J_{\mathrm{CP}}=43 \mathrm{~Hz}, \mathrm{C} 1\right.$, phosphine $), 166.58,166.62(\mathrm{C}(\mathrm{O}) \mathrm{NH}), 173.4(\mathrm{COOMe}) .{ }^{31} \mathrm{P}$ NMR $\left(\mathrm{CDCl}_{3}, 242.93 \mathrm{MHz}\right) \delta / \mathrm{ppm}:-143.96$ (septuplet, hexafluorophosphate), $21.26\left(\mathrm{~d}, 1 \mathrm{P},{ }^{2} J_{\mathrm{PP}}=\right.$ $52 \mathrm{~Hz}), 21.81\left(\mathrm{~d}, 1 \mathrm{P},{ }^{2} J_{\mathrm{PP}}=52 \mathrm{~Hz}\right)$. MALDI-HRMS $(\mathrm{m} / \mathrm{z})$ : calcd 1019.2892 $\left(\mathrm{C}_{56} \mathrm{H}_{58} \mathrm{~N}_{2} \mathrm{O}_{6} \mathrm{P}_{2} \mathrm{Ru}^{+}\right)$, found 1019.2852.


$\mathrm{mg}, 0.12 \mathrm{mmol}), \mathrm{NH}_{4} \mathrm{PF}_{6}(30.3 \mathrm{mg}, 0.19 \mathrm{mmol})$ and ligand $\mathbf{L}_{\mathbf{m G}}(100 \mathrm{mg}, 0.26 \mathrm{mmol})$. Rf (DCM/MeOH $3 \%$ ) 0.22. Yield: $46.5 \mathrm{mg}(33 \%) .{ }^{1} \mathrm{H}$ NMR (300.13 MHz, $\left.\mathrm{CDCl}_{3}\right) \delta / \mathrm{ppm}$ : $7.96(\mathrm{t}, 2 \mathrm{H}, J=5.5 \mathrm{~Hz}), 7.78(\mathrm{~d}, 2 \mathrm{H}, J=7.5 \mathrm{~Hz}), 7.2-7.54(\mathrm{~m}, 24 \mathrm{H}), 7.04(\mathrm{t}, 2 \mathrm{H}, \mathrm{J}=5 \mathrm{~Hz})$, $5.58(\mathrm{~d}, 2 \mathrm{H}, \mathrm{J}=5.5 \mathrm{~Hz}), 5.3(\mathrm{~d}, 2 \mathrm{H}, J=6 \mathrm{~Hz}), 4.17(\mathrm{~d}, 4 \mathrm{H}, \mathrm{J}=5.5 \mathrm{~Hz}), 3.79(\mathrm{~s}, 6 \mathrm{H}), 2.70$ $2.80(\mathrm{~m}, 1 \mathrm{H}), 1.23(\mathrm{~d}, 6 \mathrm{H}, \mathrm{J}=7 \mathrm{~Hz}), 1.07(\mathrm{~s}, 3 \mathrm{H}) .{ }^{13} \mathrm{C} \mathrm{NMR}\left(\mathrm{CDCl}_{3}, 150.92 \mathrm{MHz}\right) \delta / \mathrm{ppm}:$ $15.4\left(\mathrm{CH}_{3}\right.$, cymene $), 21.6\left(\mathrm{CHCH}_{3}\right.$, cymene $), 31.5\left(\mathrm{CHCH}_{3}\right.$, cymene $), 41.8(\mathrm{C} \alpha), 52.5(\mathrm{OMe})$, 89.5 (m, C2, C6, cymene), 97.5 (C4, cymene), 100.9 (C3, C5, cymene), 128.6-128.9 (m, C5, C3', phosphine), 129.6-129.7 (m, C4, phosphine), 131.2, 131.6 (C4', phosphine), 131.4 (C1, cymene), 132.5 (d, ${ }^{3} J_{\mathrm{CP}}=7 \mathrm{~Hz}, \mathrm{C} 3$, phosphine), 133.5-133.9 (C1, C1', phosphine), 134-134.6 (C2, C2', phosphine), 136.94, $137.0\left(\mathrm{~d},{ }^{2} J_{\mathrm{CP}}=4 \mathrm{~Hz}, \mathrm{C} 6\right.$, phosphine), 166.6, $166.7(\mathrm{C}(\mathrm{O}) \mathrm{NH})$, 170.3 (COOMe). ${ }^{31} \mathrm{P}$ NMR $\left(\mathrm{CDCl}_{3}, 242.93 \mathrm{MHz}\right) \delta / \mathrm{ppm}:-143.97$ (septuplet, hexafluorophosphate), 21.95 (s, 1P). +ESI MS (m/z): $1025.3\left(\mathrm{M}^{+}, 23 \%\right)$. MALDI-HRMS $(\mathrm{m} / z)$ : calcd $991.2579\left(\mathrm{C}_{54} \mathrm{H}_{54} \mathrm{~N}_{2} \mathrm{O}_{6} \mathrm{P}_{2} \mathrm{Ru}^{+}\right)$, found 991.2537 .

[(iPr- $\left.\left.p \mathrm{C}_{6} \mathbf{H}_{4}-\mathrm{Me}\right) \mathbf{R u C l}\left(\mathbf{P h}_{2} \mathbf{P}-\boldsymbol{m C}_{6} \mathbf{H}_{4}-\mathrm{CO}-\mathrm{Ala}-\mathrm{OMe}\right)_{2}\right] \mathbf{P F} \mathbf{F}_{6}, \mathbf{2}_{\mathrm{mA}}$. Mono complex $\mathbf{1}_{\mathrm{mA}}$ (78.9 $\mathrm{mg}, 0.11 \mathrm{mmol}), \mathrm{NH}_{4} \mathrm{PF}_{6}(27 \mathrm{mg}, 0.17 \mathrm{mmol})$ and ligand $\mathbf{L}_{\mathbf{m A}}(110 \mathrm{mg}, 0.28 \mathrm{mmol})$. $\mathrm{Rf}$ $(\mathrm{DCM} / \mathrm{MeOH} 3 \%)=0.26$. Yield: $81.2 \mathrm{mg}(60 \%) .{ }^{1} \mathrm{H}$ NMR $\left(300.13 \mathrm{MHz}, \mathrm{CDCl}_{3}\right) \delta / \mathrm{ppm}$ : $7.92-8.01(\mathrm{~m}, 2 \mathrm{H}), 7.71(\mathrm{~d}, 2 \mathrm{H}, J=7.5 \mathrm{~Hz}), 7.18-7.56(\mathrm{~m}, 24 \mathrm{H}), 6.81-6.91(\mathrm{~m}, 2 \mathrm{H}), 5.58-$ $5.62(\mathrm{~m}, 2 \mathrm{H}), 5.21-5.30(\mathrm{~m}, 4 \mathrm{H}), 4.62-4.72(\mathrm{~m}, 2 \mathrm{H}), 3.79(\mathrm{~d}, 6 \mathrm{H}, J=4 \mathrm{~Hz}), 2.69-2.78(\mathrm{~m}$, $1 \mathrm{H}), 1.51\left(\mathrm{dd}, 6 \mathrm{H}, J_{1}=7 \mathrm{~Hz}, J_{2}=1 \mathrm{~Hz}\right), 1.21-1.25(\mathrm{~m}, 6 \mathrm{H}), 1.11(\mathrm{~s}, 3 \mathrm{H}) .{ }^{13} \mathrm{C} \mathrm{NMR}\left(\mathrm{CDCl}_{3}\right.$, 
$75.48 \mathrm{MHz}) \delta / \mathrm{ppm}: 15.5\left(\mathrm{CH}_{3}\right.$, cymene), 18.17, $18.22(\mathrm{C} \beta), 21.5,21.6\left(\mathrm{CHCH}_{3}\right.$, cymene), $31.5\left(\mathrm{CHCH}_{3}\right.$, cymene), 48.79, $48.81(\mathrm{C} \alpha), 52.7(\mathrm{OMe}), 89.68,89.80,89.85,89.95(\mathrm{~m}, \mathrm{C} 2$, C6, cymene), 97.4, 97.5 (d, ${ }^{2} J_{\mathrm{CP}}=2.5 \mathrm{~Hz}, \mathrm{C} 3$, C5, cymene), 101.2 (C4, cymene), 128.6, 128.7 (C5, phosphine), 128.9 (d, ${ }^{3} J_{\mathrm{CP}}=16.5 \mathrm{~Hz}, \mathrm{C} 3$ ', phosphine), 129.3, 129.5 (C4, phosphine), 131.4, 131.6, 131.7 (C4', phosphine), 131.8 (C1, cymene), 132.3, 132.9 (C1', phosphine), 133.4-133.7 (m, C2, phosphine), 133.8 (C1, phosphine), 134.0 (d, ${ }^{3} J_{\mathrm{CP}}=14.5$ Hz, C3', phosphine), 134.4 (C3, phosphine),134.5-134.8 (m, C2', phosphine), 136.9-137.3 (C6, phosphine), $166.0(\mathrm{C}(\mathrm{O}) \mathrm{NH}), 173.6,173.7$ (COOMe). ${ }^{31} \mathrm{P} \mathrm{NMR}\left(\mathrm{CDCl}_{3}, 242.93 \mathrm{MHz}\right)$ $\delta / \mathrm{ppm}$ : -143.84 (septuplet, hexafluorophosphate), $22.08\left(\mathrm{~d}, 1 \mathrm{P},{ }^{2} J_{\mathrm{PP}}=52 \mathrm{~Hz}\right), 22.40(\mathrm{~d}, 1 \mathrm{P}$, $\left.{ }^{2} J_{\mathrm{PP}}=52 \mathrm{~Hz}\right) .+$ ESI MS $(\mathrm{m} / \mathrm{z}): 1053.3\left(\mathrm{M}^{+}, 3 \%\right)$. MALDI-HRMS $(\mathrm{m} / \mathrm{z})$ : calcd 1019.2892 $\left(\mathrm{C}_{56} \mathrm{H}_{58} \mathrm{~N}_{2} \mathrm{O}_{6} \mathrm{P}_{2} \mathrm{Ru}^{+}\right)$, found 1019.2852.

[(iPr-pC $\left.\left.\mathbf{C}_{6} \mathbf{H}_{4}-\mathrm{Me}\right) \mathbf{R u C l}\left(\mathbf{P h}_{2} \mathrm{P}_{-} \mathbf{C}_{2} \mathbf{H}_{4}-\mathbf{C O}-\mathrm{Gly}-\mathrm{OMe}\right)_{2}\right] \mathbf{P F} \mathbf{F}_{6}, \mathbf{2}_{\mathrm{aG}}$. Mono complex $\mathbf{1}_{\mathrm{aG}}(81.1 \mathrm{mg}$, $0.13 \mathrm{mmol}), \mathrm{NH}_{4} \mathrm{PF}_{6}(32.1 \mathrm{mg}, 0.20 \mathrm{mmol})$ and ligand $\mathbf{L}_{\mathbf{a G}}(104 \mathrm{mg}, 0.32 \mathrm{mmol})$. Rf $(\mathrm{DCM} / \mathrm{MeOH} 5 \%)=0.42$. Yield: $60.9 \mathrm{mg}(44 \%)$. As mentioned in the discussion, this compound could not be isolated with stratifying purity. ${ }^{1} \mathrm{H}$ NMR $\left(300.13 \mathrm{MHz}, \mathrm{CDCl}_{3}\right)$ $\delta /$ ppm: 1.22 (d, $J=7 \mathrm{~Hz}, 6 \mathrm{H}), 1.84-1.95(\mathrm{~m}, 4 \mathrm{H}), 2.49$ (ws, 2H), 2.75 (septuplet, $J=7 \mathrm{~Hz}$, $1 \mathrm{H}), 3.03-3.15(\mathrm{~m}, 2 \mathrm{H}), 3.72(\mathrm{~s}, 3 \mathrm{H}), 3.91(\mathrm{~d}, J=5.5 \mathrm{~Hz}, 4 \mathrm{H}), 5.18(\mathrm{~d}, J=6 \mathrm{~Hz}, 2 \mathrm{H}), 5.63-$ $5.67(\mathrm{~m}, 2 \mathrm{H}), 6.06(\mathrm{t}, J=5 \mathrm{~Hz}, 2 \mathrm{H}), 7.36-7.63(\mathrm{~m}, 20 \mathrm{H}) \cdot{ }^{13} \mathrm{C} \mathrm{NMR}\left(\mathrm{CDCl}_{3}, 150.92 \mathrm{MHz}\right)$ $\delta /$ ppm:14.7 $\left(\mathrm{CH}_{3}\right.$, cymene), $21.5\left(\mathrm{CHCH}_{3}\right.$, cymene), 22.3-22.5 (m, $\mathrm{C} 1$ phosphine), $31.0(\mathrm{C} 2$ phosphine), $31.6\left(\mathrm{CHCH}_{3}\right.$, cymene), $41.4(\mathrm{C} \alpha), 52.4(\mathrm{OMe}), 87.4\left(\mathrm{t},{ }^{2} J_{\mathrm{CP}}=5 \mathrm{~Hz}, \mathrm{C} 2, \mathrm{C} 6\right.$, cymene), 97.1 (C4, cymene), 97.7 (C3, C5, cymene), 129.3 (d, ${ }^{2} J_{\mathrm{CP}}=46 \mathrm{~Hz}, \mathrm{C} 1$ ', phosphine), 129.30, 129.34, 129.71, $129.75\left(\mathrm{~d},{ }^{3} J_{\mathrm{CP}}=5 \mathrm{~Hz}, \mathrm{C} 3{ }^{\prime}\right.$ phosphine), 131.6, 131.9 (C4, phosphine), 132.16, 132.19, 132.95, $132.98\left(\mathrm{~d},{ }^{2} J_{\mathrm{CP}}=4 \mathrm{~Hz}, \mathrm{C} 2{ }^{\prime}\right.$ phosphine), 132.8 (C1,cymene), 170.3 (COOMe), 171.14, $171.18\left(\mathrm{~d},{ }^{3} J_{\mathrm{CP}}=6 \mathrm{~Hz}, \mathrm{C}(\mathrm{O}) \mathrm{NH}\right) .{ }^{31} \mathrm{P} \mathrm{NMR}\left(\mathrm{CDCl}_{3}\right.$, $242.93 \mathrm{MHz}$ ) $\delta / \mathrm{ppm}:-143.91$ (septuplet, hexafluorophosphate), 20.41 (s, 1P).

[(iPr- $\left.\left.p \mathrm{C}_{6} \mathbf{H}_{4}-\mathrm{Me}\right) \mathbf{R u C l}\left(\mathbf{P h}_{2} \mathbf{P}-\mathbf{C}_{2} \mathbf{H}_{4}-\mathbf{C O}-A l a-O M e\right)_{2}\right] \mathbf{P F}_{6}, \mathbf{2}_{\mathrm{aA}}$. Mono complex $\mathbf{1}_{\mathrm{aA}}(65.5 \mathrm{mg}$, $0.10 \mathrm{mmol}), \mathrm{NH}_{4} \mathrm{PF}_{6}(24.9 \mathrm{mg}, 0.15 \mathrm{mmol})$ and ligand $\mathbf{L}_{\mathbf{a A}}(85 \mathrm{mg}, 0.25 \mathrm{mmol})$. $\mathrm{Rf}$ (DCM/MeOH $5 \%$ ) 0.45. Yield: $64.1 \mathrm{mg}(42 \%)$. As mentioned in the discussion, this compound could not be isolated with stratifying purity. ${ }^{1} \mathrm{H}$ NMR $\left(300.13 \mathrm{MHz}, \mathrm{CDCl}_{3}\right)$ $\delta / \mathrm{ppm}: 1.22$ (d, $J=7 \mathrm{~Hz}, 6 \mathrm{H}), 1.84-1.95(\mathrm{~m}, 4 \mathrm{H}), 2.49$ (ws, 2H), 2.75 (septuplet, $J=7 \mathrm{~Hz}$, $1 \mathrm{H}), 3.03-3.15(\mathrm{~m}, 2 \mathrm{H}), 3.72(\mathrm{~s}, 3 \mathrm{H}), 3.91(\mathrm{~d}, J=5.5 \mathrm{~Hz}, 4 \mathrm{H}), 5.18(\mathrm{~d}, J=6 \mathrm{~Hz}, 2 \mathrm{H}), 5.63-$ $5.67(\mathrm{~m}, 2 \mathrm{H}), 6.06(\mathrm{t}, J=5 \mathrm{~Hz}, 2 \mathrm{H}), 7.36-7.63(\mathrm{~m}, 20 \mathrm{H})$.

Computational details. All calculations were performed using the Gaussian09 program package (see references section). Geometries were optimized using the wB97xD density functional (Chai and Head-Gordon 2008) in conjunction with the 6-31G(d,p) for the first-row elements and $6-31+\mathrm{G}(\mathrm{d}, \mathrm{p})$ basis set for phosphorus and chlorine, while the SDD effective core potential (Andrae et al 1990) was employed for ruthenium. The nature of the stationary points was verified by vibrational analysis at the optimized geometries and no imaginary frequencies were obtained. Total Gibbs energies (Gtot) were calculated by summing electronic energies with Gibbs energy correction as obtained from the calculations with default calculation settings and without any scaling of the vibrational frequencies. Visualization of the optimized structures was done by MOLDEN 5.0. (Schaftenaar and Noordik 2000).

Biological testing. All examined organometallic ruthenium complexes were dissolved in DMSO, $(c=10 \mathrm{mM})$, and stored at $-20^{\circ} \mathrm{C}$. Just before use, these stock solutions were diluted with growth medium to the appropriate concentrations; only concentrations of the complexes 
below 33.3 $\mu \mathrm{M}$ were used, with the highest DMSO concentration well under $0.5 \%$. Ethacrynic acid (ETA; Sigma-Aldrich, USA), N-Benzyloxycarbonyl-Val-Ala-Asp(O-Me) fluoromethyl ketone (Z-VAD-FMK; Fisher Scientific, USA) and Bafilomycin A1 (BAF A; InvivoGen, USA) were dissolved in DMSO (Sigma-Aldrich) and kept at $-20{ }^{\circ} \mathrm{C}$. Caspase 3/7-Glo ${ }^{\circledR}$ Assay System (Promega, USA) was dissolved according to producer instruction and kept at $-20{ }^{\circ} \mathrm{C}$. Buthionine sulfoximine (BSO; Sigma-Aldrich), N-acetylcysteine (NAC; Sigma-Aldrich) and probenecid were dissolved in water and kept at $-20^{\circ} \mathrm{C}$. 3-(4,5-dimethyl2-thiazolyl)-2,5-diphenyl-2H-tetrazolium bromide (MTT) was purchased by Sigma-Aldrich, dissolved in phosphate-buffered saline and kept by $4{ }^{\circ} \mathrm{C}$.

Cell culture. Human cervical carcinoma (HeLa) and laryngeal carcinoma (HEp2) cells were obtained from cell culture bank (GIBCO BRL-Invitrogen, USA). Large cell lung carcinoma (H460) and human breast adenocarcinoma (MDA-MB-213) cells were obtained from American Type Culture Collection (ATCC, USA). Normal human skin keratinocyte were obtained from the foreskin of healthy boys, aged 3-8 years. Foreskin samples were noninflamed and the children were free of any therapy at least 1 month before the surgery. The cells were obtained at the Neurochemical Laboratory, Department of Chemistry and Biochemistry, School of Medicine, University of Zagreb (Gabrilovac et al 2004). Normal human skin fibroblasts were isolated from the upper arm of a 7-years-old female donor at the Neurochemical Laboratory, Department of Chemistry and Biochemistry, School of Medicine, University of Zagreb. They were used for the cytotoxicity assay at 35 and 40 population doublings. All cell lines were grown as a monolayer culture in Dulbecco's modified Eagle's medium (DMEM; Sigma-Aldrich, USA), supplemented with $10 \%$ fetal bovine serum (FBS; Sigma-Aldrich) in a humidified atmosphere of $5 \% \mathrm{CO}_{2}$ at $37{ }^{\circ} \mathrm{C}$ and were sub-cultured every 3-4 days.

Cytotoxic assay. Cytotoxic activity of newly synthesized organometallic ruthenium complexes was determined by MTT assay (Mickisch et al 1990) modified accordingly. In short, the cells were seeded into 96-well tissue culture plates. The next day, different concentrations of compounds were added to each well in quadruplicate. Upon $72 \mathrm{~h}$ incubation at $37^{\circ} \mathrm{C}$, the medium was aspirated, and the MTT dye (Sigma-Aldrich) was added. Three hours later, the formed formazan crystals were dissolved in DMSO, the plates were mechanically agitated for $5 \mathrm{~min}$ and the optical density at $545 \mathrm{~nm}$ was determined on a microtiter plate reader (Awareness Technology Inc., USA). Sulforhodamine B (SRB; SigmaAldrich) was used in order to perform SRB assay according to the protocol (Skehan et al 1990). In short, cultures fixed with trichloroacetic acid were stained for 30 minutes with $0.4 \%$ (wt/vol) SRB dissolved in 1\% acetic acid. Unbound dye was removed by four washes with $1 \%$ acetic acid, and protein-bound dye was extracted with $10 \mathrm{mM}$ unbuffered Tris base [tris (hydroxymethyl)aminomethane] for determination of optical density in a computerinterfaced, 96-well microtiter plate reader (560 nm, Awareness Technology Inc.). The percent of cell survival for each tested concentration of the compounds was calculated according to the absorption value of non-treated control cells, which was set as $100 \%$.

Potential biomolecular target evaluation. Polynucleotide and protein were purchased as noted: calf thymus (ct)-DNA (Sigma) and BSA (bovine serum albumin, Sigma), and dissolved in sodium cacodylate buffer, $\mathrm{pH}=7.0, \mathrm{I}=0.05 \mathrm{M}$. The calf thymus $c t$-DNA was additionally sonicated and filtered through a $0.45 \mathrm{~mm}$ filter (Chaires et al 1982). The $c t$-DNA concentration was determined spectroscopically at $260 \mathrm{~nm}$ using a molar extinction

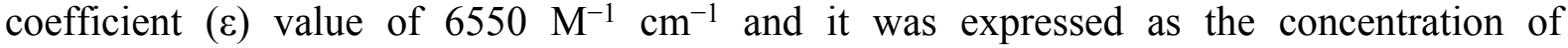
phosphates (Bresloff and Crothers 1981). 
All examined organometallic ruthenium complexes were dissolved in DMSO $\left(\mathrm{c}=10^{-2} \mathrm{M}\right.$ or $\mathrm{c}=10^{-3} \mathrm{M}$ ), and stored in refrigerator. Stock solutions were diluted with buffer during the experiment or immediately before. The highest DMSO content in solution was $\leq 1 \%$. Refrigerated DMSO stock solutions were stable longer than a month, while refrigerated DMSO solutions diluted with buffer were checked to be stable for more than one week. Concentrations of ruthenium complexes below $2 \times 10^{-5} \mathrm{M}$ were used to avoid intermolecular association. At given experimental conditions the absorbance of measured compounds was proportional to their concentrations.

Thermal melting curves for $c t$-DNA and its complexes with studied organometallic ruthenium complexes were determined following the absorption change at $260 \mathrm{~nm}$ as a function of temperature (Mergny and Lacroix 2003). Absorbance scale was normalized. Tm values were the midpoints of the transition curves determined from the maximum of the first derivative and checked graphically by the tangent method. The $\Delta \mathrm{Tm}$ values were calculated by subtracting $\mathrm{Tm}$ value of the free polynucleotide from $\mathrm{Tm}$ value of the complex. Every $\Delta \mathrm{Tm}$ value was the average of at least two measurements. The error in $\Delta \mathrm{Tm}$ is $\pm 0.5^{\circ} \mathrm{C}$.

CD titrations were performed by adding portions of the compound stock solution into the solution of polynucleotide $\left(\mathrm{c}=2 \times 10^{-5} \mathrm{M}\right)$ or protein $\left(\mathrm{c}=2 \times 10^{-6} \mathrm{M}\right)$. Scanning speed was $200 \mathrm{~nm} \mathrm{~min}{ }^{-1}$, the buffer background was subtracted from each spectrum, while each spectrum was a result of three accumulations.

Fluorimetric titrations were performed by adding aliquots of organometallic ruthenium complexes stock solution into the buffered solution of the BSA protein and by monitoring fluorescence of protein. Excitation wavelength of $\lambda_{\mathrm{exc}}=300 \mathrm{~nm}$ was used to avoid absorption of excitation light caused by increasing absorbance of the organometallic ruthenium complex. After mixing protein with studied compounds it was observed that equilibrium was reached in less than 120 seconds. Due to low concentrations of studied compounds and protein used in fluorimetric titrations no precipitation occurred. Emission was collected in the range $\lambda_{\mathrm{em}}=320-450 \mathrm{~nm}$. Titration data were processed by non-linear least-square fitting program SPECFIT (Gampp et al 1985, Maeder and Zuberbuhler 1990) that gave the best fit of 1:1 stoichiometry of complexes. The binding constants of complexes of examined compounds with BSA protein were calculated for the concentration range corresponding to ca. $20-80 \%$ complexation.

Cell cycle analysis. HeLa cells were seeded into tissue culture plates and treated with either $10 \mu \mathrm{M}$ of $\mathbf{2}_{\mathrm{mG}}$ during $24-72 \mathrm{~h}$ or different concentrations of the compound during $72 \mathrm{~h}$, in order to analyze the cell cycle progression. Thereafter, both adherent and floating cells were collected, washed with PBS, fixed in $70 \%$ ethanol and left overnight at $-20^{\circ} \mathrm{C}$. Fixed cells were treated with RNase A $(0.1 \mathrm{mg} / \mathrm{mL}$, Sigma-Aldrich) for one hour at room temperature and afterward stained with propidium iodide $(50 \mu \mathrm{g} / \mathrm{mL}$, Sigma-Aldrich) for $30 \mathrm{~min}$ in the dark. The DNA content was analyzed by flow cytometry NaviosTM (Beckman Coulter, Miami, Fl, USA). Data were analyzed with FlowLogic software (Inivai Technologies, Victoria, Australia). Propidium iodide stained samples were analyzed using Watson pragmatic algorithm for modeling cell cycle data.

Cell death analysis. Twenty-four hours after the seeding, HeLa cells were treated either with 10 and $16 \mu \mathrm{M}$ concentrations of $\mathbf{2}_{\mathrm{mG}}$ during $24-72 \mathrm{~h}$ or with different concentrations of $\mathbf{2}_{\mathbf{m G}}$ during $72 \mathrm{~h}$. Starved or heat shocked cells were always used as a positive (compensation) 
controls. After indicated time point, both adherent and floating cells were collected, centrifuged and washed with PBS. The cell suspension was incubated with Annexin V-FITC (BD Biosciences, USA; according to producer's protocol) and propidium iodide $(5 \mu \mathrm{g} / \mathrm{mL}$, Sigma-Aldrich). Upon $30 \mathrm{~min}$ incubation at room temperature in dark, the viable, early apoptotic, late apoptotic/necrotic and necrotic cell populations were detected and counted by flow cytometry NaviosTM (Beckman Coulter, Miami, Fl, USA). Data were analyzed with FlowLogic software (Inivai Technologies, Victoria, Australia).

Twenty-four hours after the seeding, HeLa cells were treated with $22 \mu \mathrm{M} \mathbf{2}_{\mathbf{m G}}$. The total cell lysates were collected 24-72 $\mathrm{h}$ upon treatment and loaded onto a $10 \%$ SDS polyacrylamide gel and run for two hours at $35 \mathrm{~mA}$. Separated proteins were transferred onto a $0.2 \mathrm{~mm}$ nitrocellulose membrane (Schleicher and Schull, Germany) in a Bio-Rad blot cell (Bio-Rad, USA), using buffer consisting of $25 \mathrm{mM}$ Tris/HCl, $86 \mathrm{mM}$ glycine and $20 \%$ methanol. To avoid nonspecific binding, the membrane was incubated in blocking buffer ( $5 \%$ non fat dry milk, $0.1 \%$ Tween 20 in PBS) for one hour at room temperature and then incubated with antiPARP (Cell Signaling Technology, USA), Beclin-1 (Santa Cruz Biotechnology, USA) or LCR-I/II antibody (MBL, USA) at room temperature for two hours. After washing the membrane with $0.1 \%$ Tween 20 in TBS and incubation with corresponding horseradish peroxidase-coupled secondary antibody (Amersham Pharmacia Biotech, Germany), the proteins were visualized with ECL (Perkin Elmer, USA) according to the manufacturer's protocol. All membranes were incubated with $\beta$-Tubulin, which was used as a loading control (Sigma-Aldrich, USA). The role of caspases in $\mathbf{2}_{\mathbf{m G}}$ induced cell death was investigated by MTT assay. HeLa cells were pre-treated for two hours with $6.25 \mu \mathrm{M}$ specific pan-caspase inhibitor, Z-VAD. Upon pre-treatment, different concentrations of $\mathbf{2}_{\mathbf{m G}}$ were added and the cytotoxicity effect was determined $72 \mathrm{~h}$ later as described above.

The role of autophagy in $\mathbf{2}_{\mathbf{m G}}$ induced cell death was also investigated by MTT assay. HeLa cells were pre-treated for two hours with $0.5 \mathrm{nM}$ specific inhibitor of autophagy, BAF A. Upon pre-treatment, different concentrations of $\mathbf{2}_{\mathbf{m G}}$ were added and the cytotoxicity effect was determined $72 \mathrm{~h}$ later as described above.

Determination of glutathione role in cell response. The role of intracellular GSH in cell response to $\mathbf{2}_{\mathbf{p G}}, \mathbf{2}_{\mathbf{p A}}, \mathbf{2}_{\mathbf{m G}}$ and $\mathbf{2}_{\mathrm{mA}}$ was investigated by MTT assay. HeLa cells were either pre-treated overnight with specific inhibitor of GSH synthesis, $0.01 \mu \mathrm{g} / \mathrm{mL}$ BSO or for two hours with precursor in GSH synthesis, $5 \mathrm{mM}$ NAC. Upon pre-treatment, different concentrations of indicated organometallic complexes were added and the cytotoxicity effect was determined $72 \mathrm{~h}$ later as described above. The capacity of GSH to form the detoxification conjugates through enzymatic reaction with $\mathbf{2}_{\mathbf{m G}}$ was investigated by treatment of HeLa cells with combination of 5 or $7.5 \mu \mathrm{g} / \mathrm{mL}$ ETA (the inhibitor of glutathione S-transferase; the enzyme involved in reaction of GSH with compounds) and different concentrations of $\mathbf{2}_{\mathbf{m G}}$. The cell survival was examined $72 \mathrm{~h}$ after. The optimal concentrations of used modulators of GSH synthesis and glutathione S-transferase reaction were determined previously (Brozovic et al 2008, Brozovic et al 2013, Osmak and Eljuga 1993). The activity of GSH pumps to efflux the GSH-2 ${ }_{\text {mG }}$ conjugates was examined by pre-treatment of HeLa cells for one hour with 0.2 or $0.625 \mathrm{mM}$ probenecid, what was followed by treatment of HeLa cells with different concentrations of $\mathbf{2}_{\mathbf{m G}}$. Seventy-two hours after, the effect of combination treatment to $\mathbf{2}_{\mathbf{m G}}$ treatment alone was determined.

Determination of total cell ruthenisation. Total cell ruthenisation was measured as described previously for measurement of total cell platination (Brozovic et al 2013) with 
modifications. Briefly, the cells were treated with either $150 \mu \mathrm{M}$ of $\mathbf{2}_{\mathbf{p G}}, \mathbf{2}_{\mathbf{p A}}, \mathbf{2}_{\mathbf{m G}}$ and $\mathbf{2}_{\mathbf{m A}}$ during 2-6 h or 17.5-150 $\mu \mathrm{M} \mathbf{2}_{\mathbf{p G}}, \mathbf{2}_{\mathbf{p A}}, \mathbf{2}_{\mathbf{m G}}$ and $\mathbf{2}_{\mathbf{m A}}$ during $2 \mathrm{~h}$, rinsed with ice-cold PBS, and harvested into $10 \mathrm{ml}$ of ice-cold PBS using a rubber policeman. After centrifugation, the cells were re-suspended in PBS, an aliquot was used for determination of cell number, and the remainder was digested in $70 \%$ nitric acid. Cell lysates were heated for $2 \mathrm{~h}$ at $75{ }^{\circ} \mathrm{C}$, diluted to $5 \%$ nitric acid, and assayed for ruthenium content. The amount of ruthenium was measured by a validated high-resolution inductively coupled plasma mass spectrometry (HR ICPMS) using the Element 2 (Thermo Finnigan, Germany). Calibration standards were prepared from $\mathrm{RuCl}_{3} \times 3 \mathrm{H}_{2} \mathrm{O}$ diluted in 1:4 hydrochloric acid and water $(1000 \mu \mathrm{g} / \mathrm{mL}$; Agilent, USA).

Statistical analysis. Data were analyzed by Student's t-test, and expressed as the mean \pm standard error of the mean. Data were considered significant when $\mathrm{P}$ values were lower than 0.05 , and in the figures these are designated as $*=\mathrm{P}<0.05$ or $* *=\mathrm{P}<0.01$. Experiments were performed in triplicate and repeated at least twice.

Acknowledgement. These materials are based on work financed by the Croatian Science Foundation (CSF, project numbers IP-2014-09-1461, IP-2016-06-1036 and DOK-2018-018086), the Terry Fox Foundation, Croatian League Against Cancer and in part by a CroatianSlovenian bilateral project. The authors would like to thank Professor Maja Osmak and Dr. Mario Cindrić (both Ruđer Bošković Institute) for helpful comments, Ernest Sanders (Ruđer Bošković Institute) for technical assistance and COST Action CM1105 for financial support.

\section{References.}

Adhireksan Z, Davey GE, Campomanes P, Groessl M, Clavel CM, Yu HJ et al (2014). Ligand substitutions between ruthenium-cymene compounds can control protein versus DNA targeting and anticancer activity. Nature Communication 5: 1-13.

Antonarakis ES, Emadi A (2010). Ruthenium-based chemotherapeutics: are they ready for prime time? Cancer Chemotherapy and Pharmacology 66: 1-9.

Bergamo A, Sava G (2015). Linking the future of anticancer metal-complexes to the therapy of tumour metastases. Chemical Society Reviews 44: 8818-8835.

Biancalana L, Pratesi A, Chiellini F, Zacchini S, Funaioli T, Gabbiani C et al (2017). Ruthenium arene complexes with triphenylphosphane ligands: cytotoxicity towards pancreatic cancer cells, interaction with model proteins, and effect of ethacrynic acid substitution. New Journal of Chemistry 41: 14574-14588.

Blunden BM, Rawal A, Lu HX, Stenzel MH (2014). Superior Chemotherapeutic Benefits from the Ruthenium-Based Anti-Metastatic Drug NAMI-A through Conjugation to Polymeric Micelles. Macromolecules 47: 1646-1655.

Bresloff JL, Crothers DM (1981). Equilibrium Studies of Ethidium-Polynucleotide Interactions. Biochemistry 20: 3547-3553. 
Brozovic A, Osmak M (2007). Activation of mitogen-activated protein kinases by cisplatin and their role in cisplatin-resistance. Cancer Letters 251: 1-16.

Brozovic A, Majhen D, Roje V, Mikac N, Jakopec S, Fritz G et al (2008). alpha(v)beta(3) Integrin-mediated drug resistance in human laryngeal carcinoma cells is caused by glutathione-dependent elimination of drug-induced reactive oxidative species. Molecular Pharmacology 74: 298-306.

Brozovic A, Ambriovic-Ristov A, Osmak M (2010). The relationship between cisplatininduced reactive oxygen species, glutathione, and BCL-2 and resistance to cisplatin. Critical Reviews in Toxicology 40: 347-359.

Brozovic A, Vukovic L, Polancac DS, Arany I, Koberle B, Fritz G et al (2013). Endoplasmic reticulum stress is involved in the response of human laryngeal carcinoma cells to Carboplatin but is absent in Carboplatin-resistant cells. PloS One 8: e76397.

Brozovic A, Stojanovic N, Ambriovic-Ristov A, Brozovic Krijan A, Polanc S, Osmak M (2014). 3-Acetyl-bis(2-chloro-4-nitrophenyl)triazene is a potent antitumor agent that induces oxidative stress and independently activates the stress-activated protein kinase/c-Jun NH2terminal kinase pathway. Anti-cancer Drugs 25: 289-295.

Bugarcic T, Novakova O, Halamikova A, Zerzankova L, Vrana O, Kasparkova J et al (2008). Cytotoxicity, cellular uptake, and DNA interactions of new monodentate ruthenium(II) complexes containing terphenyl arenes. Journal of Medicinal Chemistry 51: 5310-5319.

Chaires JB, Dattagupta N, Crothers DM (1982). Studies on Interaction of Anthracycline Antibiotics and Deoxyribonucleic-Acid - Equilibrium Binding-Studies on Interaction of Daunomycin with Deoxyribonucleic-Acid. Biochemistry 21: 3933-3940.

Chaplin AB, Fellay C, Laurenczy G, Dyson PJ (2007). Mechanistic studies on the formation of eta(2)-diphosphine (eta(6)-p-cymene)ruthenium(II) compounds. Organometallics 26: 586593.

Cimbora-Zovko T, Brozovic A, Piantanida I, Fritz G, Virag A, Alic B et al (2011). Synthesis and biological evaluation of 4-nitro-substituted 1,3-diaryltriazenes as a novel class of potent antitumor agents. European Journal of Medicinal Chemistry 46: 2971-2983.

Dikic I, Elazar Z (2018). Mechanism and medical implications of mammalian autophagy. Nature Reviews Molecular Cell Biology 19: 349-364.

Domotor O, Hartinger CG, Bytzek AK, Kiss T, Keppler BK, Enyedy EA (2013). Characterization of the binding sites of the anticancer ruthenium(III) complexes KP1019 and 
KP1339 on human serum albumin via competition studies. Journal of biological inorganic chemistry 18: 9-17.

Dos Santos ER, Correa RS, Ribeiro JU, Graminha AE, Ellena J, Selistre-de-Araujo HS et al (2016). $\mathrm{Ru}(\mathrm{II}) /$ bisphosphine/diimine/amino acid complexes: diastereoisomerism, cytotoxicity, and inhibition of tumor cell adhesion to collagen type I. Journal of Coordination Chemistry 69: $3518-3530$.

Fischer U, Janicke RU, Schulze-Osthoff K (2003). Many cuts to ruin: a comprehensive update of caspase substrates. Cell Death and Differentiation 10: 76-100.

Gabrilovac J, Cupic B, Breljak D, Zekusic M, Boranic M (2004). Expression of CD13/aminopeptidase $\mathrm{N}$ and CD10/neutral endopeptidase on cultured human keratinocytes. Immunology Letters 91: 39-47.

Galluzzi L, Senovilla L, Vitale I, Michels J, Martins I, Kepp O et al (2012). Molecular mechanisms of cisplatin resistance. Oncogene 31: 1869-1883.

Gampp H, Maeder M, Meyer CJ, Zuberbuhler AD (1985). Calculation of EquilibriumConstants from Multiwavelength Spectroscopic Data .2. Specfit - 2 User-Friendly Programs in Basic and Standard Fortran-77. Talanta 32: 257-264.

Gasser G, Ott I, Metzler-Nolte N (2011). Organometallic Anticancer Compounds. Journal of Medicinal Chemistry 54: 3-25.

Gaussian 09, Revision E.01, Frisch MJ, Trucks GW, Schlegel HB, Scuseria GE, Robb MA, Cheeseman JR et al, Gaussian, Inc., Wallingford CT, 2009.

Gridnev ID, Imamoto T (2015). Challenging the Major/Minor Concept in Rh-Catalyzed Asymmetric Hydrogenation. ACS Catalysis 5: 2911-2915.

Kaufmann SH, Desnoyers S, Ottaviano Y, Davidson NE, Poirier GG (1993). Specific proteolytic cleavage of poly(ADP-ribose) polymerase: an early marker of chemotherapyinduced apoptosis. Cancer Research 53: 3976-3985.

Kirin SI, Schatzschneider U, de Hatten X, Weyhermuller T, Metzler-Nolte N (2006). 1,n 'disubstituted ferrocenoyl amino acids and dipeptides: Conformational analysis by $\mathrm{CD}$ spectroscopy, X-ray crystallography, and DFT calculations. Journal of Organometallic Chemistry 691: 3451-3457.

Klionsky DJ, Abdalla FC, Abeliovich H, Abraham RT, Acevedo-Arozena A, Adeli K et al (2012). Guidelines for the use and interpretation of assays for monitoring autophagy. Autophagy 8: 445-544. 
Kokan Z, Kirin SI (2012). The application of "backdoor induction" in bioinspired asymmetric catalysis. RSC Advances 2: 5729-5737.

Kokan Z, Kirin SI (2013). "Backdoor Induction" of Chirality in Asymmetric Hydrogenation with Rhodium(I) Complexes of Amino Acid Substituted Triphenylphosphane Ligands. European Journal of Organic Chemistry 2013: 8154-8161.

Kokan Z, Glasovac Z, Elenkov MM, Gredicak M, Jeric I, Kirin SI (2014). "Backdoor Induction" of Chirality: Asymmetric Hydrogenation with Rhodium(I) Complexes of Triphenylphosphane-Substituted beta-Turn Mimetics. Organometallics 33: 4005-4015.

Kokan Z, Peric B, Kovacevic G, Brozovic A, Metzler-Nolte N, Kirin SI (2017). cis-versus trans-Square-Planar Palladium(II) and Platinum(II) Complexes with Triphenylphosphine Amino Acid Bioconjugates. European Journal of Inorganic Chemistry: 3928-3937.

Kokan Z, Kovacevic B, Stefanic Z, Tzvetkova P, Kirin SI (2018). Controlling orthogonal self-assembly through cis-trans isomerization of a non-covalent palladium complex dimer. Chemical Communication 54: 2094-2097.

Komor AC, Barton JK (2013). The path for metal complexes to a DNA target. Chemical Communication 49: 3617-3630.

Levine B, Kroemer G (2008). Autophagy in the pathogenesis of disease. Cell 132: 27-42.

Li J, Yuan J (2008). Caspases in apoptosis and beyond. Oncogene 27: 6194-6206.

Lin Y, Huang Y, Zheng W, Wang F, Habtemariam A, Luo Q et al (2013). Organometallic ruthenium anticancer complexes inhibit human glutathione-S-transferase pi. Journal of Inorganic Biochemistry 128: 77-84.

Liu CY, Takemasa A, Liles WC, Goodman DB, Jonas M, Rosen H, et al (2003). Broadspectrum caspase inhibition paradox-ically augmented cell death in TNFa-stimulated neutroph-ils. Blood 101: 295-304.

Liu Z, Habtemariam A, Pizarro AM, Fletcher SA, Kisova A, Vrana O et al (2011). Organometallic half-sandwich iridium anticancer complexes. Journal of Medicinal Chemistry 54: 3011-3026.

Maeder M, Zuberbuhler AD (1990). Nonlinear Least-Squares Fitting of Multivariate Absorption Data. Analitycal Chemistry 62: 2220-2224. 
Martinez MA, Carranza MP, Massaguer A, Santos L, Organero JA, Aliende C et al (2017). Synthesis and Biological Evaluation of $\mathrm{Ru}(\mathrm{II})$ and $\mathrm{Pt}(\mathrm{II})$ Complexes Bearing Carboxyl Groups as Potential Anticancer Targeted Drugs. Inorganic Chemistry 56: 13679-13696.

Meier-Menches SM, Gerner C, Berger W, Hartinger CG, Keppler BK (2018). Structureactivity relationships for ruthenium and osmium anticancer agents - towards clinical development. Chemical Society Reviews 47: 909-928.

Mergny JL, Lacroix L (2003). Analysis of thermal melting curves. Oligonucleotides 13: 515537.

Mickisch G, Fajta S, Keilhauer G, Schlick E, Tschada R, Alken P (1990). Chemosensitivity testing of primary human renal cell carcinoma by a tetrazolium based microculture assay (MTT). Urological Research 18: 131-136.

Millan G, Gimenez N, Lara R, Berenguer JR, Moreno MT, Lalinde E et al (2019). Luminescent Cycloplatinated Complexes with Biologically Relevant Phosphine Ligands: Optical and Cytotoxic Properties. Inorganic Chemistry 58: 1657-1673.

Minenkov Y, Singstad A, Occhipinti G, Jensen VR (2012). The accuracy of DFT-optimized geometries of functional transition metal compounds: a validation study of catalysts for olefin metathesis and other reactions in the homogeneous phase. Dalton Transactions 41: 55265541 .

Nikoletopoulou V, Markaki M, Palikaras K, Tavernarakis N (2013). Crosstalk between apoptosis, necrosis and autophagy. Biochimica et Biophysica Acta 1833: 3448-3459.

Novakova O, Kasparkova J, Bursova V, Hofr C, Vojtiskova M, Chen HM et al (2005). Conformation of DNA modified by monofunctional Ru(II) arene complexes: Recognition by DNA binding proteins and repair. Relationship to cytotoxicity. Chemistry \& Biology 12: 121129.

Opačak S., Kokan Z., Glasovac Z., Perić B., Kirin, S. I. (2019) "Backdoor induction" of chirality: Trans-1,2-cyclohexanediamine as key building block for asymmetric hydrogenation catalysts, European Journal Organic Chemistry, in press, doi: 10.1002/ejoc.201801647.

Osmak M, Eljuga D (1993). The characterization of two human cervical carcinoma HeLa sublines resistant to cisplatin. Research in Experimental Medicine 193: 389-396.

Osmak M, Brozovic A, Ambriovic-Ristov A, Hadzija M, Pivcevic B, Smital T (1998). Inhibition of apoptosis is the cause of resistance to doxorubicin in human breast adenocarcinoma cells. Neoplasma 45: 223-230.

Otagiri M, Giam Chuang VS (Eds.), (2016) Albumin in Medicine. Pathological and Clinical Applications, Springer. ISBN 978-981-10-2116-9 
Pongratz M, Schluga P, Jakupec MA, Arion VB, Hartinger CG, Allmaier G et al (2004). Transferrin binding and transferrin-mediated cellular uptake of the ruthenium coordination compound KP1019, studied by means of AAS, ESI-MS and CD spectroscopy. Journal of Analytical Atomic Spectrometry 19: 46-51.

Puckett CA, Ernst RJ, Barton JK (2010). Exploring the cellular accumulation of metal complexes. Dalton Transactions 39: 1159-1170.

Qi SC, Hayashi J, Zhang L (2016). Recent application of calculations of metal complexes based on density functional theory. RSC Advances 6: 77375-77395.

Rathgeb A, Bohm A, Novak MS, Gavriluta A, Domotor O, Tornmasino JB et al (2014). Ruthenium-Nitrosyl Complexes with Glycine, L-Alanine, L-Valine, L-Proline, D-Proline, LSerine, L-Threonine, and L-Tyrosine: Synthesis, X-ray Diffraction Structures, Spectroscopic and Electrochemical Properties, and Antiproliferative Activity. Inorganic Chemistry 53: 2718-2729.

Ravera M, Baracco S, Cassino C, Colangelo D, Bagni G, Sava G et al (2004). Electrochemical measurements confirm the preferential bonding of the antimetastatic complex $[\operatorname{ImH}][\mathrm{RuCl}(\mathrm{DSMO})(\mathrm{Im})]$ (NAMI-A) with the proteins and the weak interaction with nucleobases. Journal of Inorganic Biochemistry 98: 984-990.

Peters T, Jr., (1995) All About Albumin. Biochemistry, Genetics, and Medical Applications, Elsevier Inc. ISBN: 978-0-12-552110-9

Saez R, Lorenzo J, Prieto MJ, Font-Bardia M, Calvet T, Onnenaca N et al (2014). Influence of $\mathrm{PPh} 3$ moiety in the anticancer activity of new organometallic ruthenium complexes. Journal of Inorganic Biochemistry 136: 1-12.

Sava G, Frausin F, Cocchietto M, Vita F, Podda E, Spessotto P et al (2004). Actin-dependent tumour cell adhesion after short-term exposure to the antimetastasis ruthenium complex NAMI-A. European Journal of Cancer 40: 1383-1396.

Schaftenaar G, Noordik JH (2000). Molden: a pre- and post-processing program for molecular and electronic structures. Journal of Computer-Aided Molecular Design 14: 123134.

Scolaro C, Chaplin AB, Hartinger CG, Bergamo A, Cocchietto M, Keppler BK et al (2007). Tuning the hydrophobicity of ruthenium(II)-arene (RAPTA) drugs to modify uptake, biomolecular interactions and efficacy. Dalton Transactions: 5065-5072.

Scrase TG, O'Neill MJ, Peel AJ, Senior PW, Matthews PD, Shi H et al (2015). Selective lability of ruthenium(II) arene amino acid complexes. Inorganic Chemistry 54: 3118-3124. 
Sersen S, Kljun J, Kryeziu K, Panchuk R, Alte B, Korner W et al (2015). Structure-Related Mode-of-Action Differences of Anticancer Organoruthenium Complexes with betaDiketonates. Journal of Medicinal Chemistry 58: 3984-3996.

Skehan P, Storeng R, Scudiero D, Monks A, McMahon J, Vistica D et al (1990). New colorimetric cytotoxicity assay for anticancer-drug screening. Journal of National Cancer Institute 82: 1107-1112.

Sommer MG, Kureljak P, Urankar D, Schweinfurth D, Stojanovic N, Bubrin M et al (2014). Combining [Arene-Ru] with Azocarboxamide to Generate a Complex with Cytotoxic Properties. Chemistry-a European Journal 20: 17296-17299.

Stojanovic N, Urankar D, Brozovic A, Ambriovic-Ristov A, Osmak M, Kosmrlj J (2013). Design and evaluation of biological activity of diazenecarboxamide-extended cisplatin and carboplatin analogues. Acta Chimica Slovenica 60: 368-374.

Tewari M, Quan LT, O'Rourke K, Desnoyers S, Zeng Z, Beidler DR et al (1995). Yama/CPP32 beta, a mammalian homolog of CED-3, is a CrmA-inhibitable protease that cleaves the death substrate poly(ADP-ribose) polymerase. Cell 81: 801-809.

Townsend DM, Tew KD (2003). The role of glutathione-S-transferase in anti-cancer drug resistance. Oncogene 22: 7369-7375.

Vajs J, Steiner I, Brozovic A, Pevec A, Ambriovic-Ristov A, Matkovic M et al (2015). The 1,3-diaryltriazenido(p-cymene)ruthenium(II) complexes with a high in vitro anticancer activity. Journal of Inorganic Biochemistry 153: 42-48.

Vandenabeele P, Grootjans S, Callewaert N, Takahashi N (2013). Necrostatin-1 blocks both RIPK1 and IDO: consequences for the study of cell death in experimental disease models. Cell Death and Differentiation 20: 185-187.

Wang FY, Xu JJ, Habtemariam A, Bella J, Sadler PJ (2005). Competition between glutathione and guanine for a ruthenium(II) arene anticancer complex: Detection of a sulfenato intermediate. Journal of the American Chemical Society 127: 17734-17743.

Wu Y-T, Tan H-L, Shui G, Bauvy C, Huang Q, Wenk MR et al (2010). Dual role of 3methyladenine in modulation of autophagy via different temporal patterns of inhibition on class I and III phosphoinositide 3-kinase. The Journal of Biological Chemistry 285: 1085010861.

Zaman GJ, Lankelma J, van Tellingen O, Beijnen J, Dekker H, Paulusma C et al (1995). Role of glutathione in the export of compounds from cells by the multidrug-resistance-associated 
protein. Proceedings of the National Academy of Sciences of the United States of America 92: 7690-7694. 


\section{Organometallic ruthenium(II)-arene complexes with triphenylphosphine amino acid bioconjugates: Synthesis, characterization and biological properties}


Piantanida, ${ }^{\mathrm{c}}$ Domagoj Eljuga, ${ }^{\mathrm{d}}$ Iztok Turel, ${ }^{\mathrm{e}}$ Anamaria Brozovic, ${ }^{\mathrm{a}, *}$ Srećko I. Kirin, ${ }^{\mathrm{b}, *}$

a Division of Molecular Biology, Ruđer Bošković Institute, Bijenička cesta 54, HR-10000 Zagreb, Croatia

b Division of Materials Chemistry, Ruđer Bošković Institute, Bijenička cesta 54, HR-10000 Zagreb, Croatia

${ }^{\mathrm{c}}$ Division of Organic Chemistry and Biochemistry, Ruđer Bošković Institute, Bijenička cesta 54, HR-10000 Zagreb, Croatia

d Department for Oncoplastic and Reconstructive Surgery, University Hospital for Tumors, University Clinical Hospital Centre Sisters of Mercy, HR-10000 Zagreb, Croatia

e Faculty of Chemistry and Chemical Technology, University of Ljubljana, Večna pot 113, SLO-1000 Ljubljana, Slovenia

\# equal contribution

* corresponding authors

E-mail: Anamaria.Brozovic@irb.hr, Srecko.Kirin@irb.hr

Key words: Ruthenium, $p$-Cymene, Phosphine ligands, Cytotoxicity, Cell death 
Contents

NMR Measurements $\quad 2$

$\begin{array}{lr}\text { CD Measurements } & 26\end{array}$

$\begin{array}{lr}\text { DFT Calculations } & 27\end{array}$

Biomacromolecular target evaluation $\quad 28$

$\begin{array}{ll}\text { Biological evaluation } & 33\end{array}$ 
NMR Measurements.

Table S1. Chosen ${ }^{1} \mathrm{H}$ NMR shifts $\left(\mathrm{CDCl}_{3}, \delta / \mathrm{ppm}\right.$ (integral)).*

\begin{tabular}{|l|l|c|c|c|c|c|c|c|}
\hline No. & Description & \multicolumn{3}{|c|}{ NH } & \multicolumn{2}{c|}{$\mathbf{C H}_{3}$ cymene } & \multicolumn{2}{c|}{ Ph cymene } \\
\hline & & $\mathbf{L}$ & $\mathbf{M L}$ & $\mathbf{M L}_{2}$ & $\mathbf{M L}$ & $\mathbf{M L}_{2}$ & $\mathbf{M L}$ & $\mathbf{M L}_{2}$ \\
\hline pG & pGly-OMe & 6.63 & 6.64 & 7.12 & 1.86 & 1.10 & $4.98(2)$ & $5.14(2)$ \\
& & & & & & & $5.22(2)$ & $5.52(2)$ \\
\hline pA & pAla-OMe & 6.70 & 6.67 & 7.03 & 1.87 & 1.08 & $4.98(2)$ & $5.09(1)$ \\
& & & & & & & $5.23(2)$ & $5.24(1)$ \\
& & & & & & & & $5.55(2)$ \\
\hline mG & mGly-OMe & 6.53 & 6.93 & 7.04 & 1.88 & 1.07 & $5.07(2)$ & $5.30(2)$ \\
& & & & & & & $5.22(2)$ & $5.58(2)$ \\
\hline mA & mAla-OMe & 6.61 & 6.89 & 6.85 & 1.89 & 1.11 & $4.96(1)$ & $5.22(1)$ \\
& & & & 6.87 & & & $5.16(2)$ & $5.28(1)$ \\
& & & & & & & $5.27(1)$ & $5.60(2)$ \\
\hline aG & alifGly-OMe & 5.93 & 6.21 & 6.06 & 1.90 & 0.79 & $5.09(2)$ & $5.18(2)$ \\
& & & & & & & $5.18(2)$ & $5.65(2)$ \\
\hline aA & alifAla-OMe & 5.97 & 6.14 & 5.86 & 1.64 & 0.80 & $5.09(2)$ & $5.14(1)$ \\
& & & & 5.98 & & & $5.19(2)$ & $5.21(1)$ \\
& & & & & & & & $5.62(1)$ \\
& & & & & & & & $5.68(1)$ \\
\hline
\end{tabular}

* The C2 proton of the substituted aromatic ring of the ligand is unexpectedly highly deshielded in $\mathbf{1} \mathbf{c}, \mathbf{d}$ complexes $(\delta \approx 8.9 \mathrm{ppm})$. 
${ }^{1} \mathrm{H}$ NMR $\left(\mathrm{CDCl}_{3}\right)$ spectrum of $\mathbf{L}_{\mathbf{m G}}$.
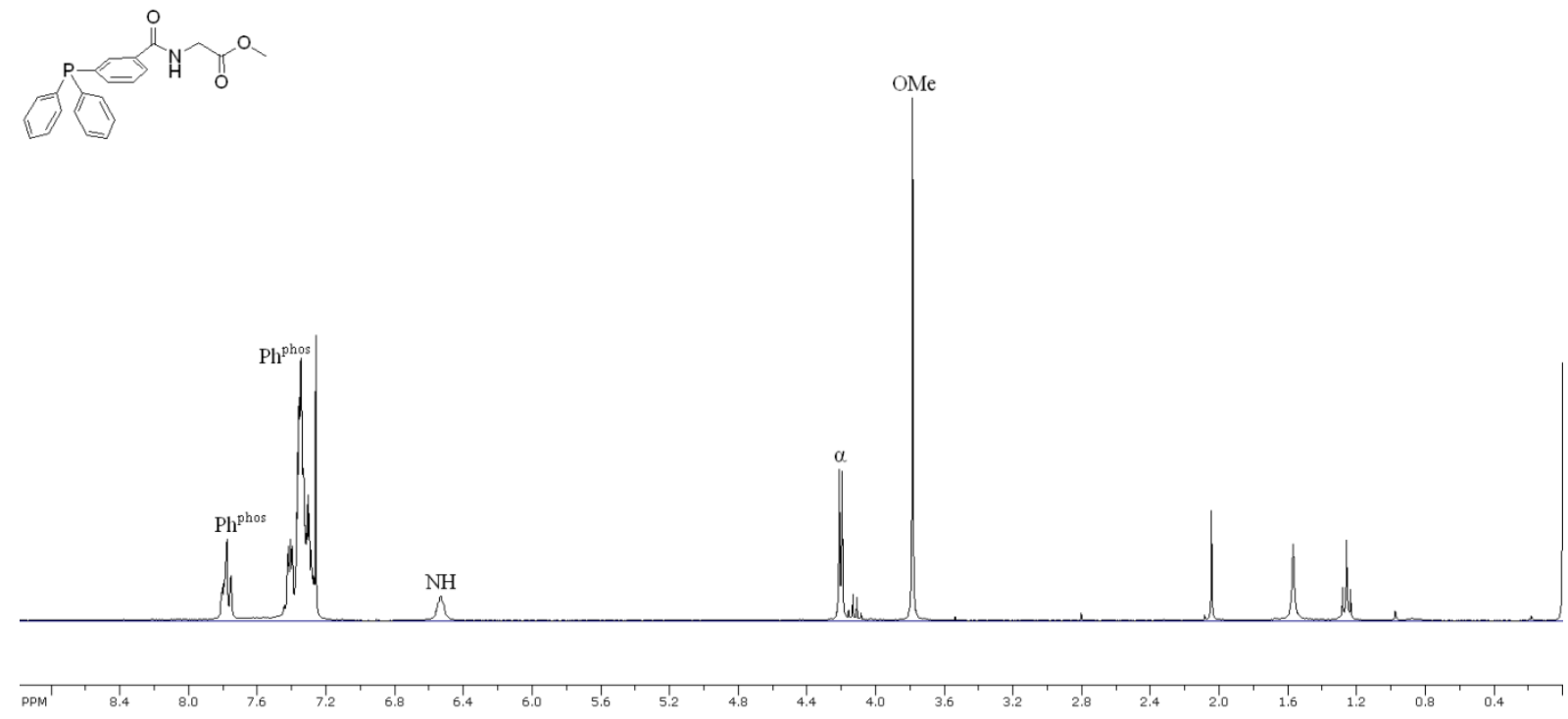

${ }^{13} \mathrm{C}$ NMR $\left(\mathrm{CDCl}_{3}\right)$ spectrum of $\mathbf{L}_{\mathbf{m G}}$.
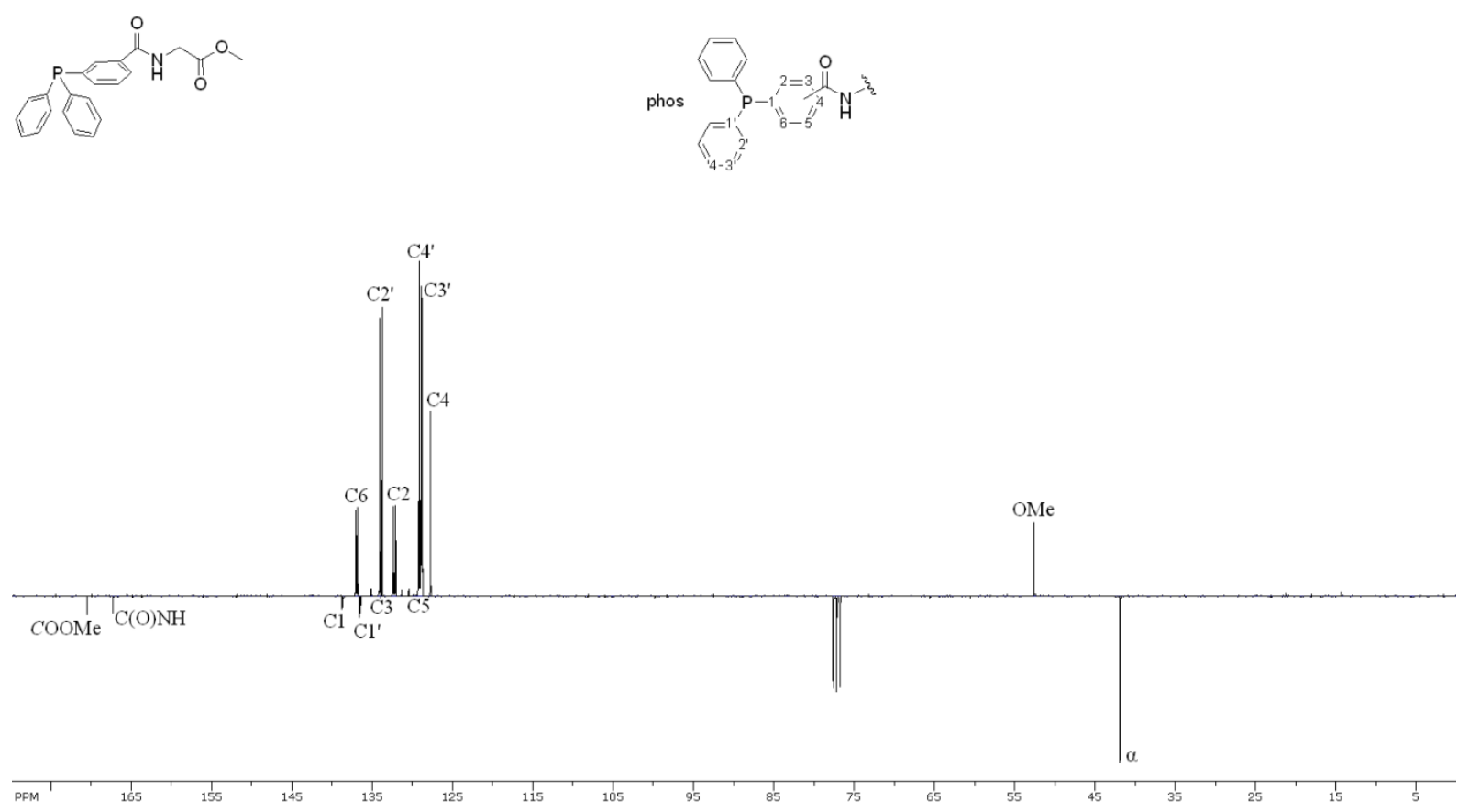
${ }^{1} \mathrm{H}$ NMR $\left(\mathrm{CDCl}_{3}\right)$ spectrum of $\mathbf{L}_{\mathbf{a G}}$.


${ }^{13} \mathrm{C}$ NMR $\left(\mathrm{CDCl}_{3}\right)$ spectrum of $\mathbf{L}_{\mathbf{a G}}$.

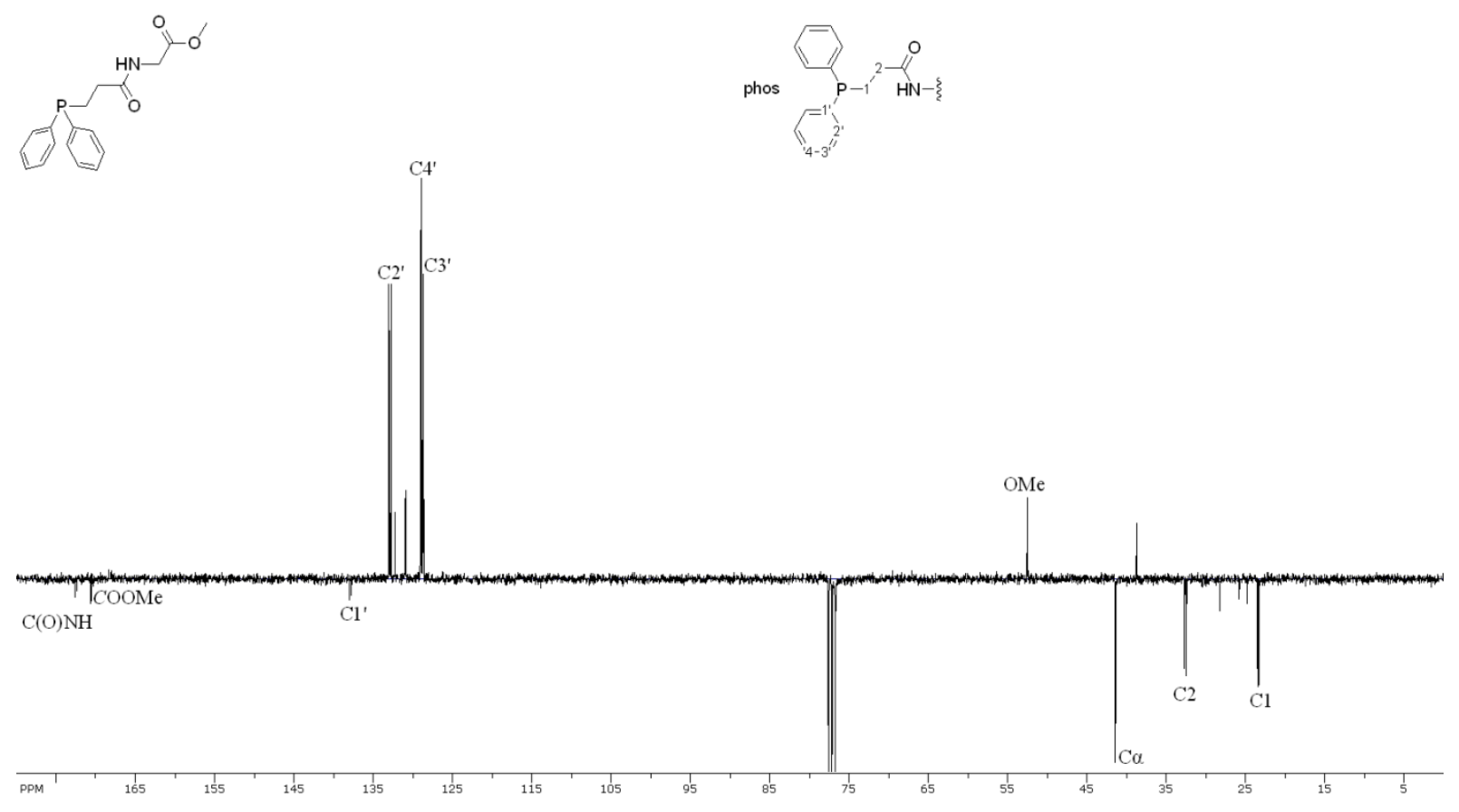


${ }^{1} \mathrm{H}$ NMR $\left(\mathrm{CDCl}_{3}\right)$ spectrum of $\mathbf{L}_{\mathbf{a A}}$.
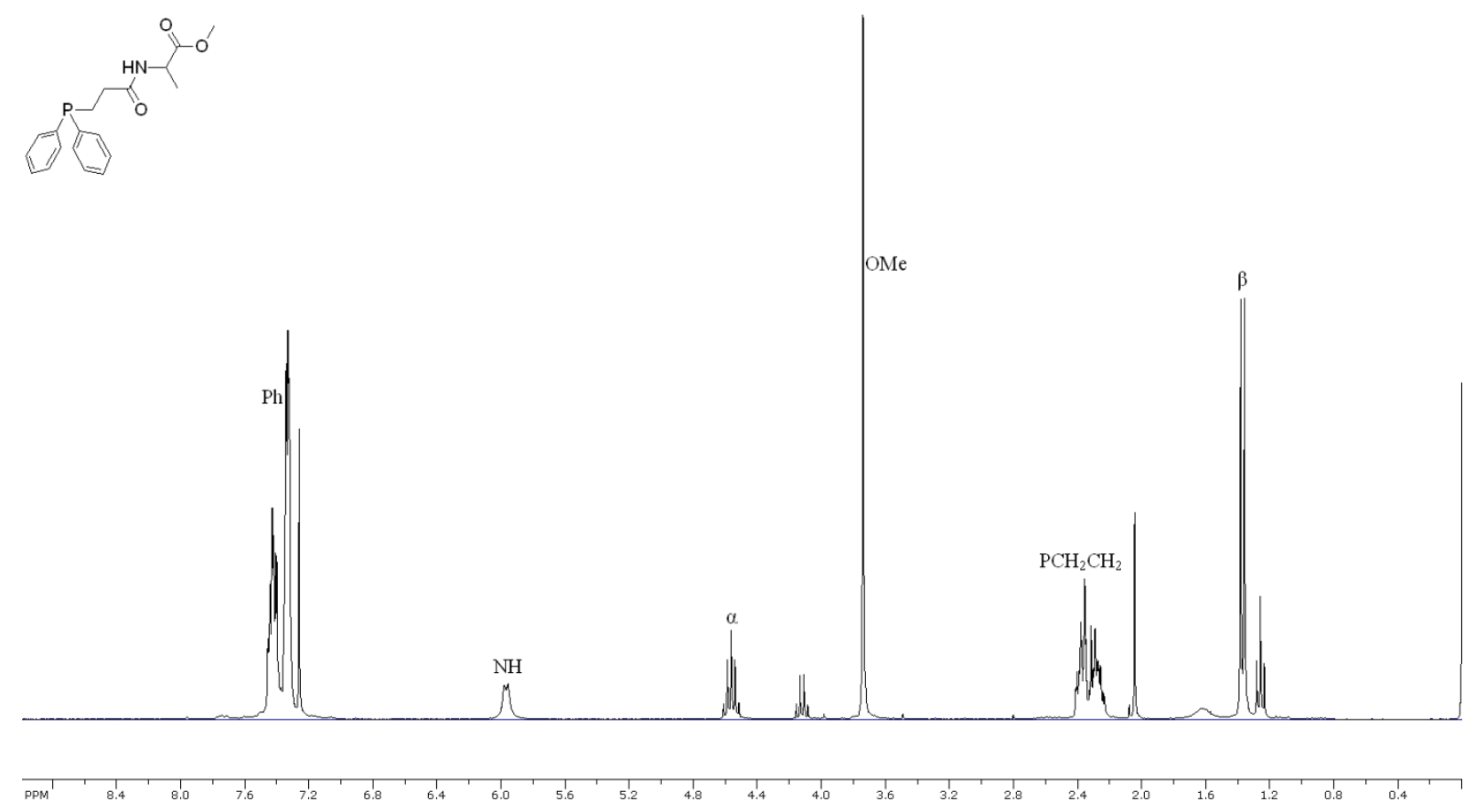

${ }^{13} \mathrm{C}$ NMR $\left(\mathrm{CDCl}_{3}\right)$ spectrum of $\mathbf{L}_{\mathbf{a A}}$.
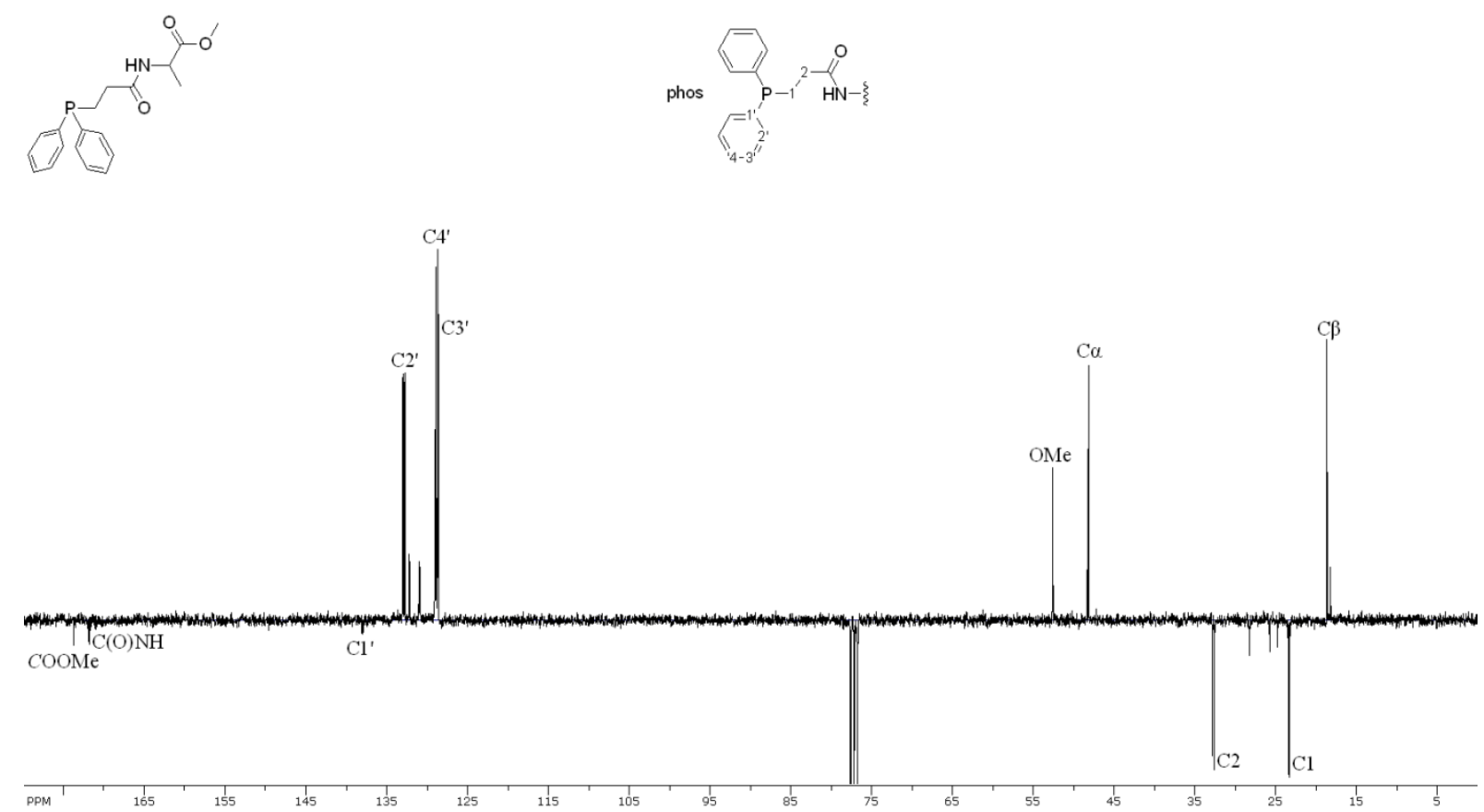
${ }^{1} \mathrm{H}$ NMR $\left(\mathrm{CDCl}_{3}\right)$ spectrum of $\mathbf{1}_{\mathbf{p G}}$.


${ }^{13} \mathrm{C} \mathrm{NMR}\left(\mathrm{CDCl}_{3}\right)$ spectrum of $\mathbf{1}_{\mathbf{p G}}$.
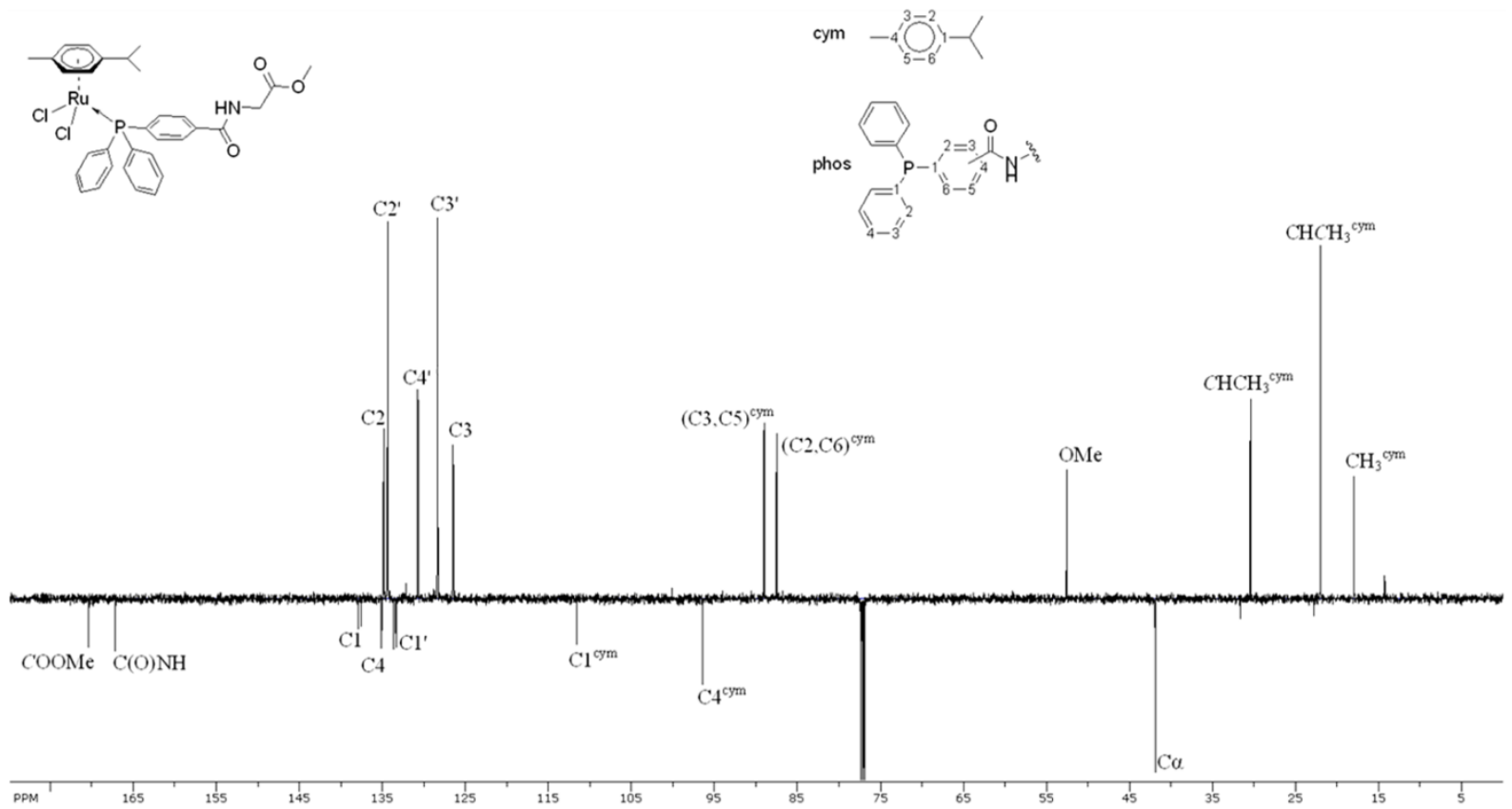
${ }^{1} \mathrm{H}-{ }^{13} \mathrm{C}$ HMQC NMR $\left(\mathrm{CDCl}_{3}\right)$ spectrum of $\mathbf{1}_{\mathbf{p G}}$.

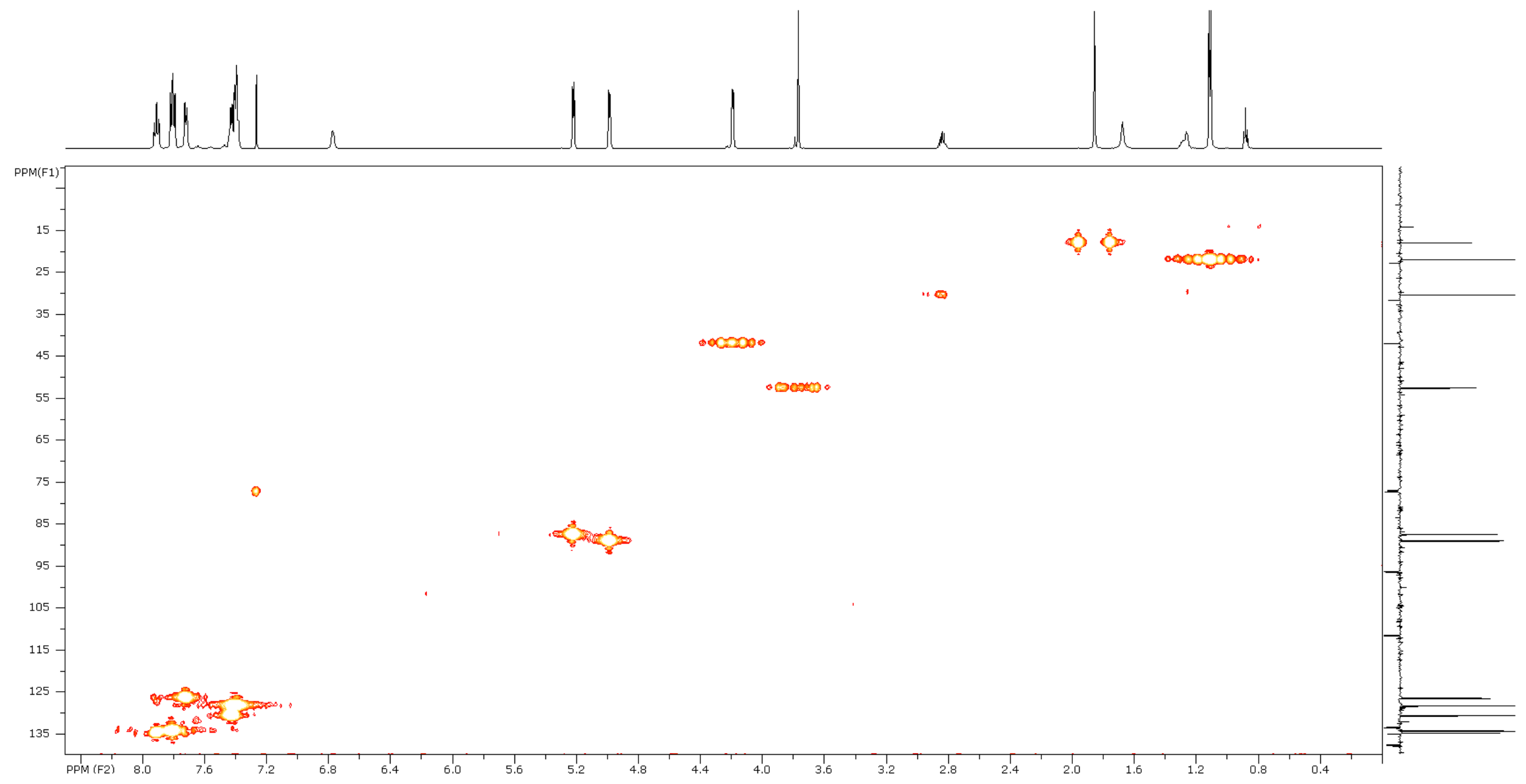


${ }^{1} \mathrm{H}-{ }^{13} \mathrm{C} \mathrm{HMBC}$ NMR $\left(\mathrm{CDCl}_{3}\right)$ spectrum of $\mathbf{1}_{\mathbf{p G}}$.






$$
-5
$$


${ }^{1} \mathrm{H}$ NOESY $\left(\mathrm{CDCl}_{3}\right)$ spectrum of $\mathbf{1}_{\mathbf{m G}}$.

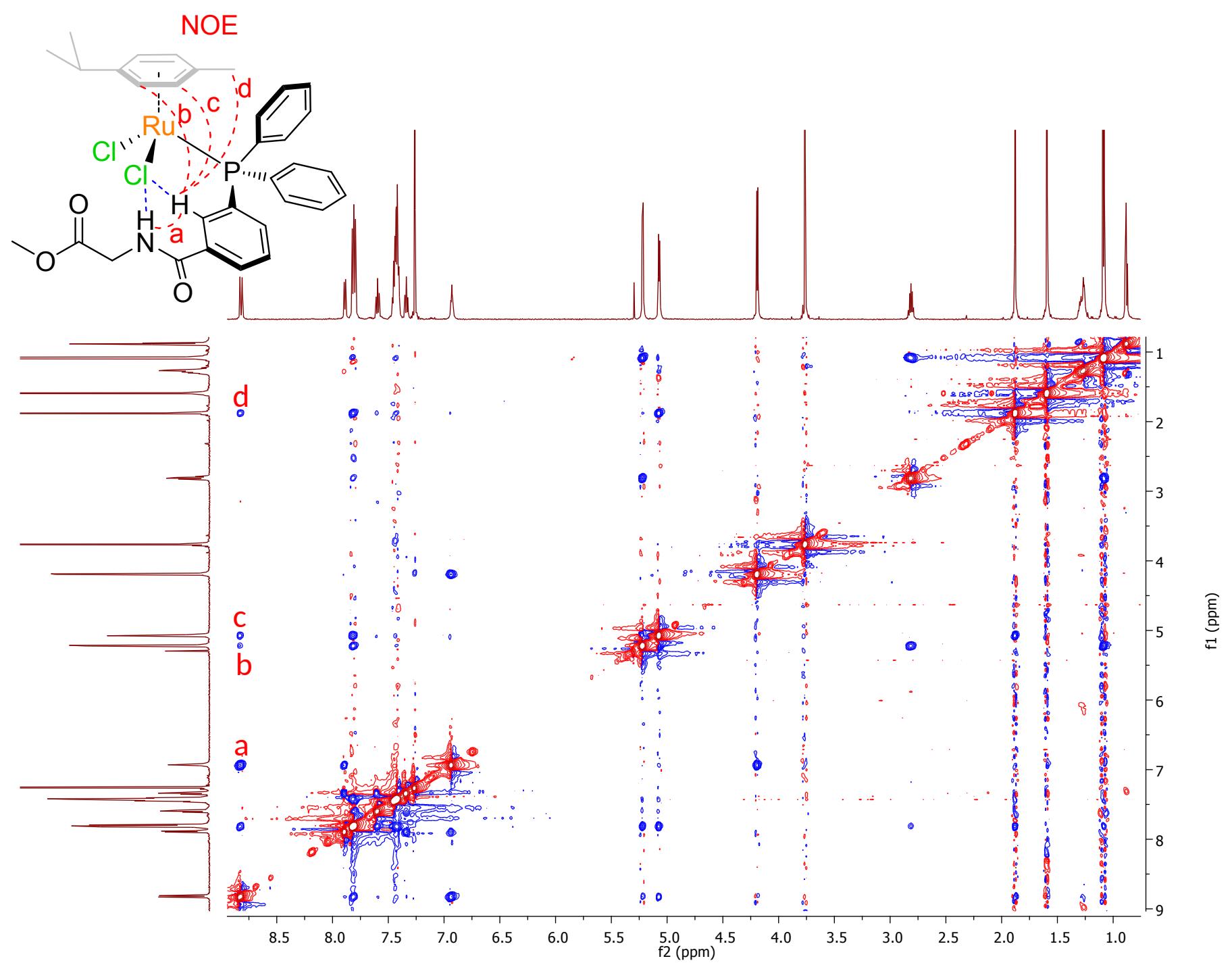


${ }^{1} \mathrm{H}$ NMR $\left(\mathrm{CDCl}_{3}\right)$ spectrum of $\mathbf{1}_{\mathbf{p A}}$.
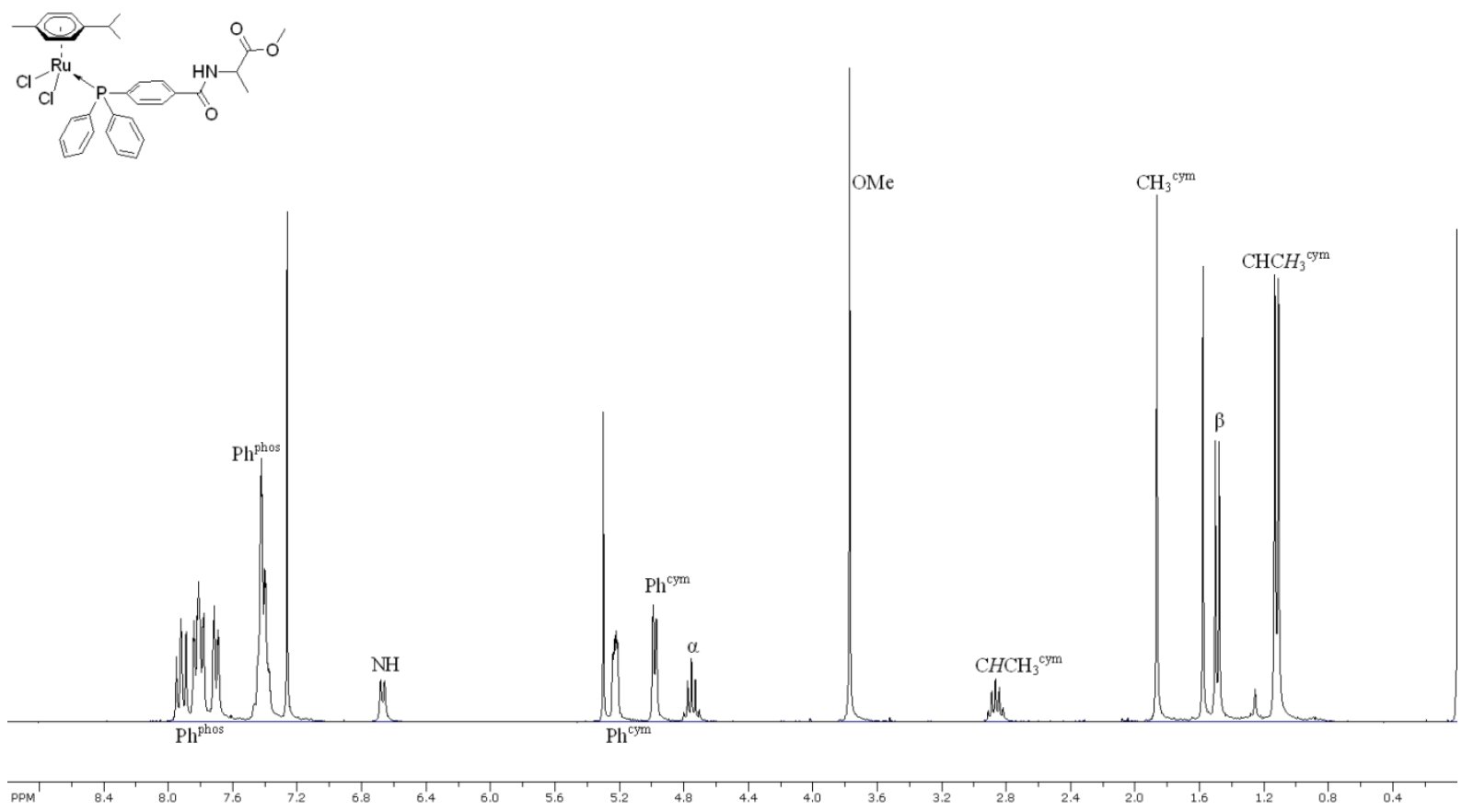

${ }^{13} \mathrm{C} \mathrm{NMR}\left(\mathrm{CDCl}_{3}\right)$ spectrum of $\mathbf{1}_{\mathbf{p A}}$

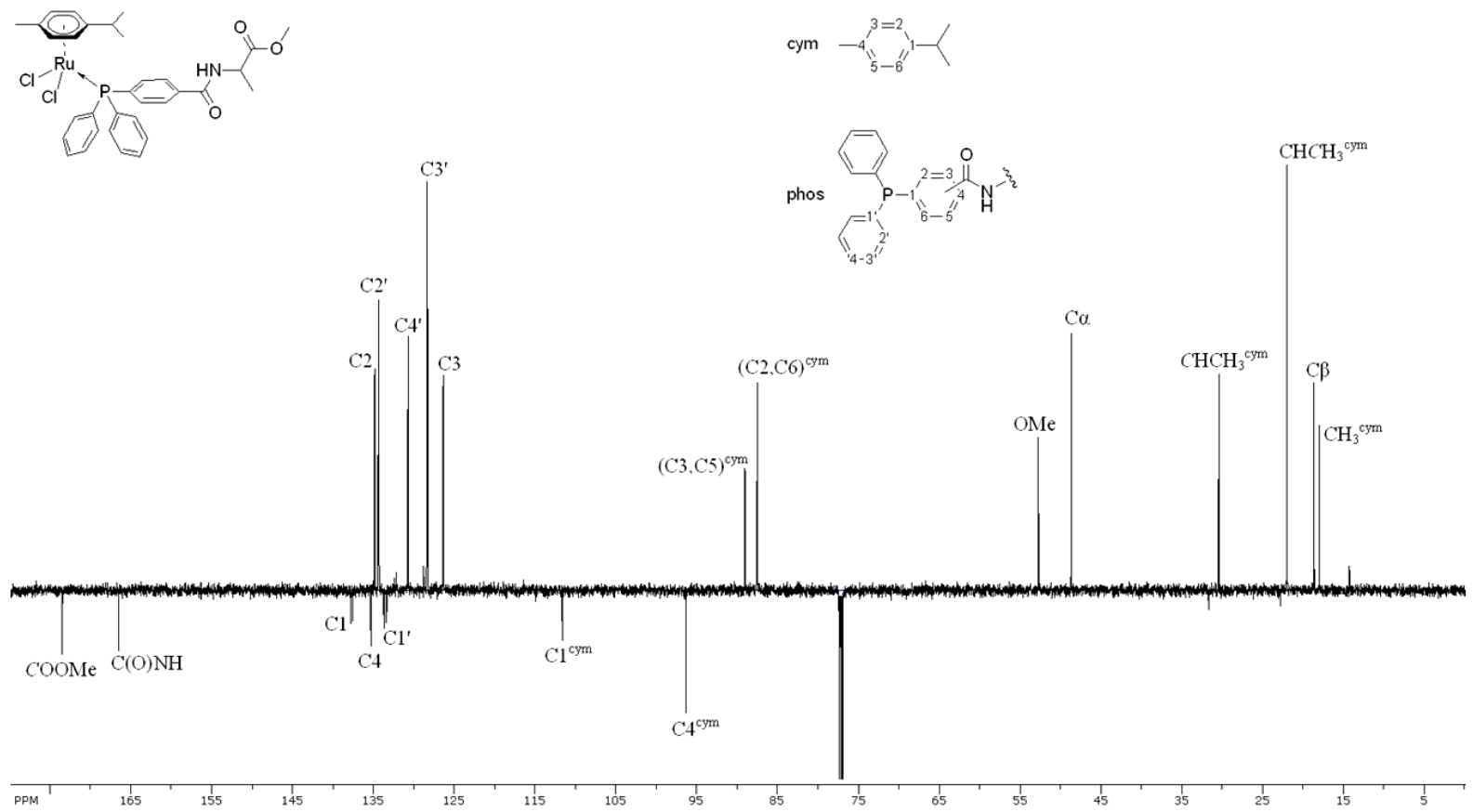


${ }^{1} \mathrm{H} \mathrm{NMR}\left(\mathrm{CDCl}_{3}\right)$ spectrum of $\mathbf{1}_{\mathbf{m G}}$.
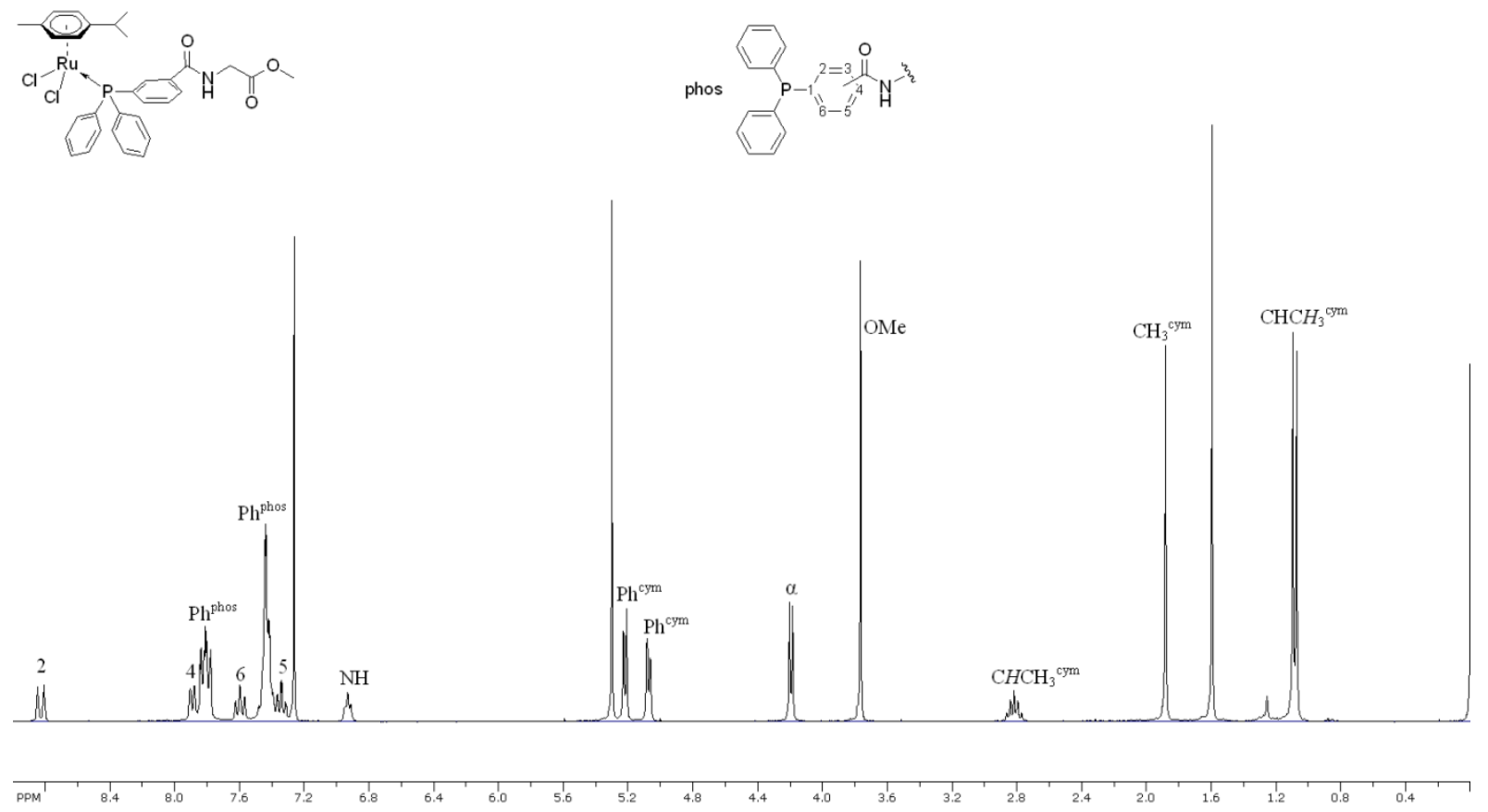

${ }^{13} \mathrm{C}$ NMR $\left(\mathrm{CDCl}_{3}\right)$ spectrum of $\mathbf{1}_{\mathbf{m G}}$
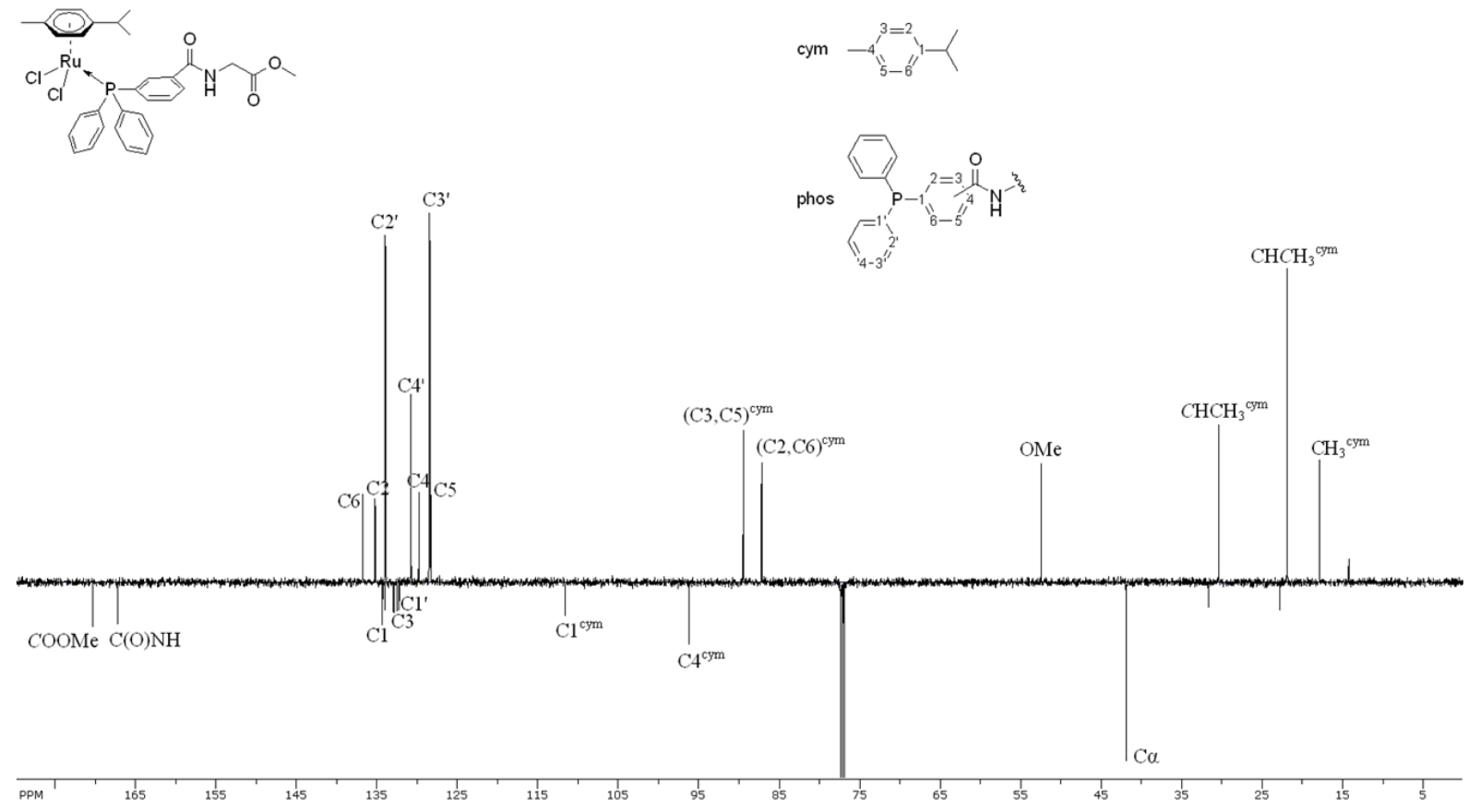
${ }^{1} \mathrm{H} \mathrm{NMR}\left(\mathrm{CDCl}_{3}\right)$ spectrum of $\mathbf{1}_{\mathbf{m A}}$.
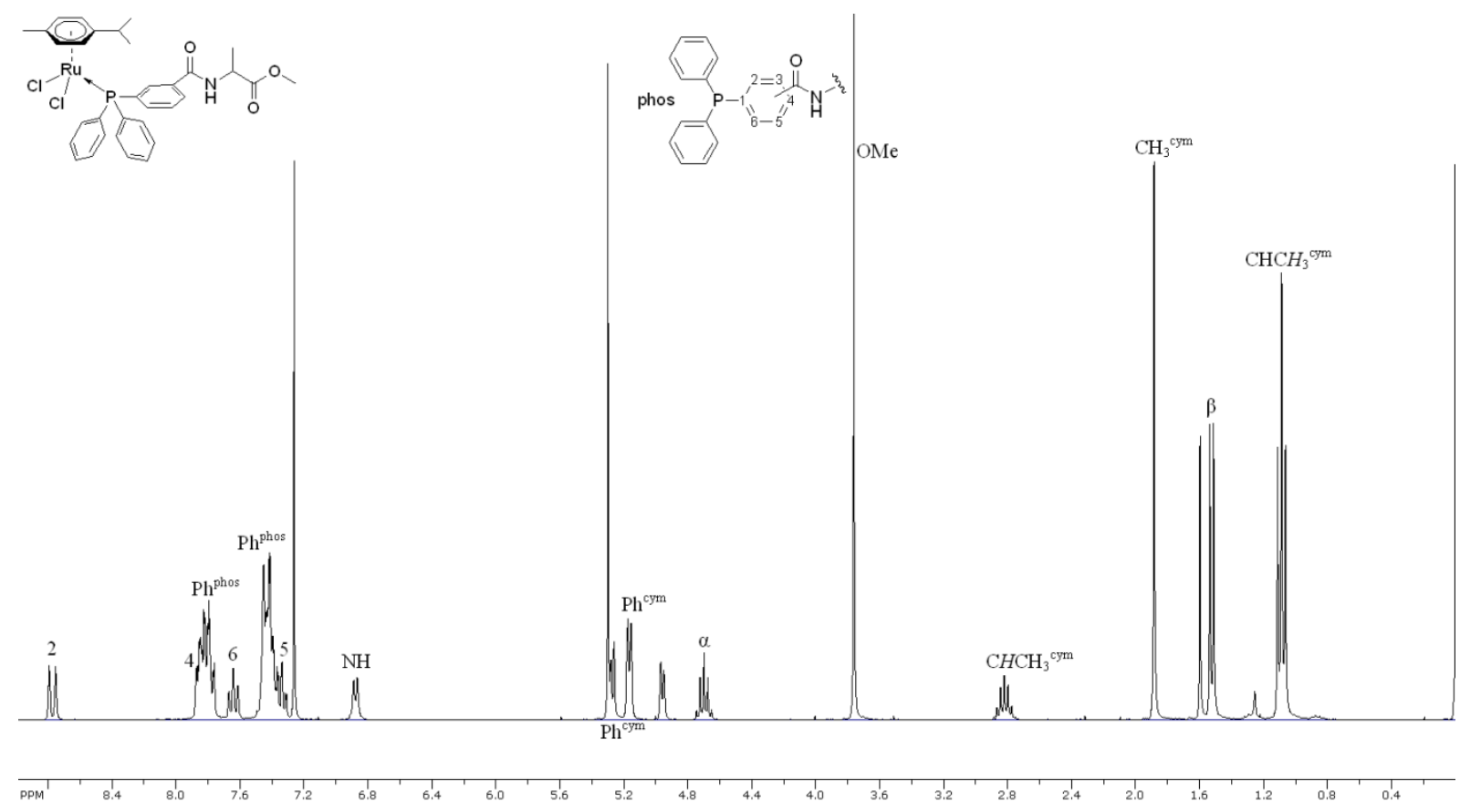

${ }^{13} \mathrm{C} \mathrm{NMR}\left(\mathrm{CDCl}_{3}\right)$ spectrum of $\mathbf{1}_{\mathbf{m A}}$.
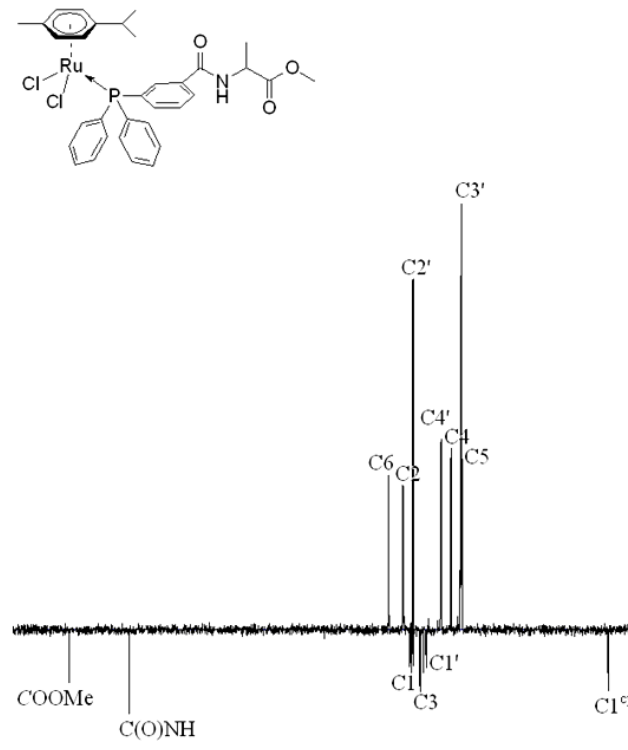

cym

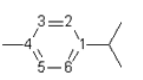

$(\mathrm{C} 3, \mathrm{C} 5)^{\mathrm{cym}}(\mathrm{C} 2, \mathrm{C} 6)^{\mathrm{cym}}$

phos

$\mathrm{CHCH}_{3}$
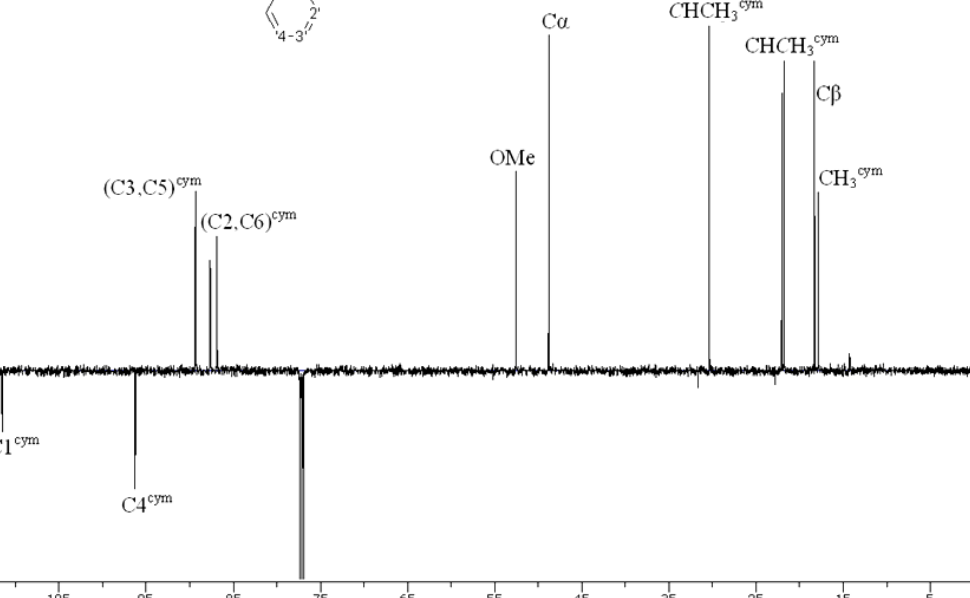
${ }^{1} \mathrm{H}$ NMR $\left(\mathrm{CDCl}_{3}\right)$ spectrum of $\mathbf{1}_{\mathbf{a G}}$.
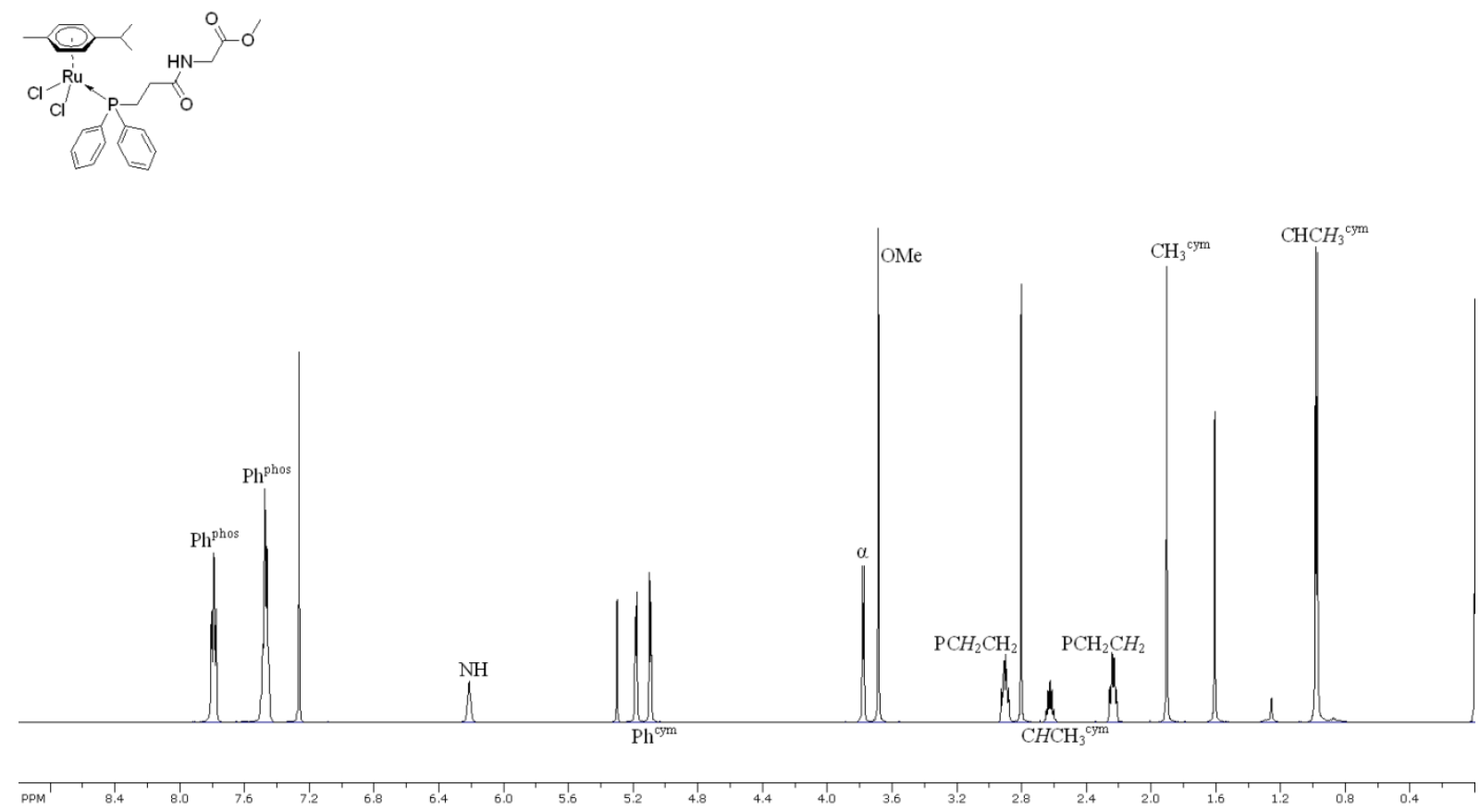

${ }^{13} \mathrm{C} \mathrm{NMR}\left(\mathrm{CDCl}_{3}\right)$ spectrum of $\mathbf{1}_{\mathbf{a G}}$.

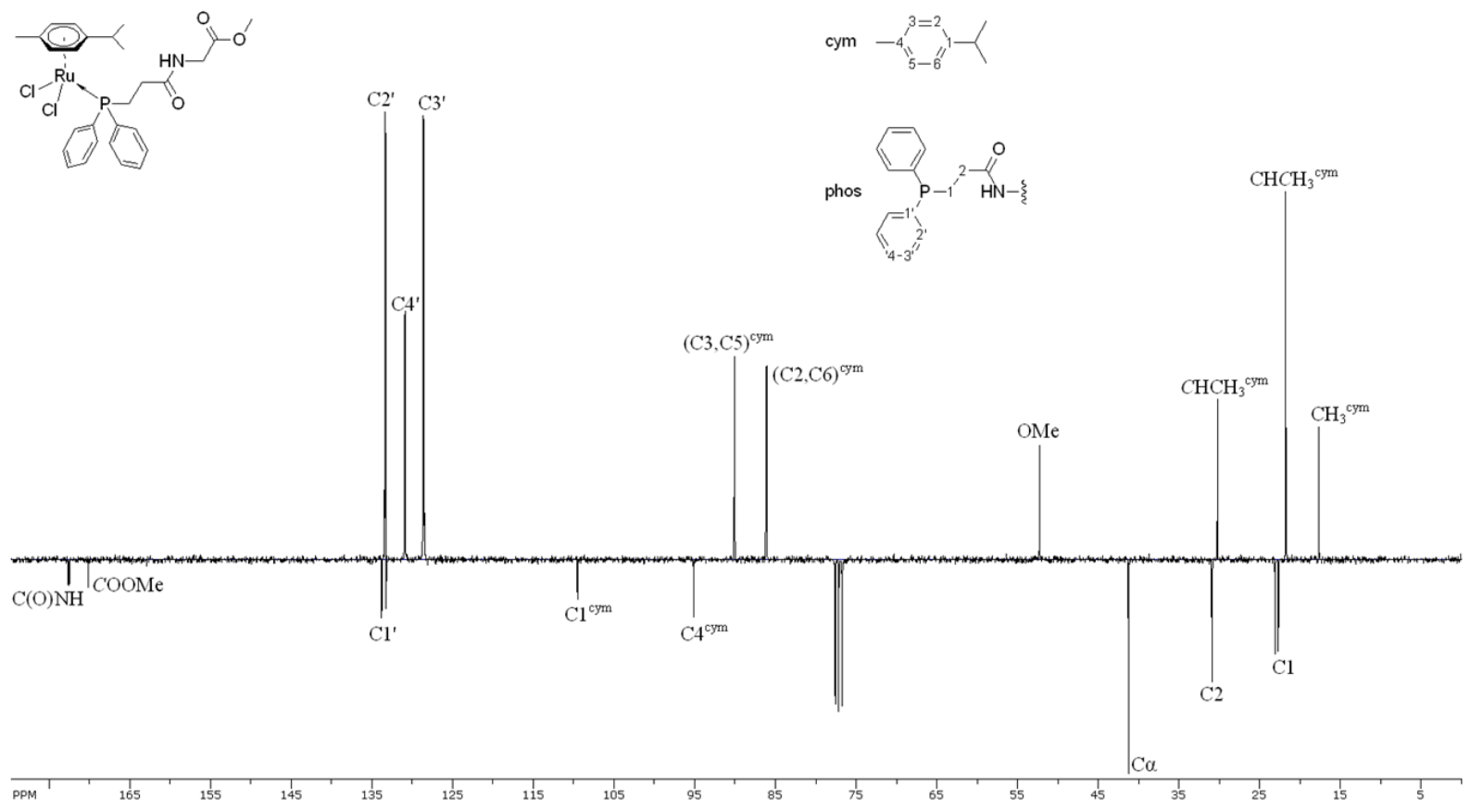


${ }^{1} \mathrm{H}$ NMR $\left(\mathrm{CDCl}_{3}\right)$ spectrum of $\mathbf{1}_{\mathrm{aA}}$.
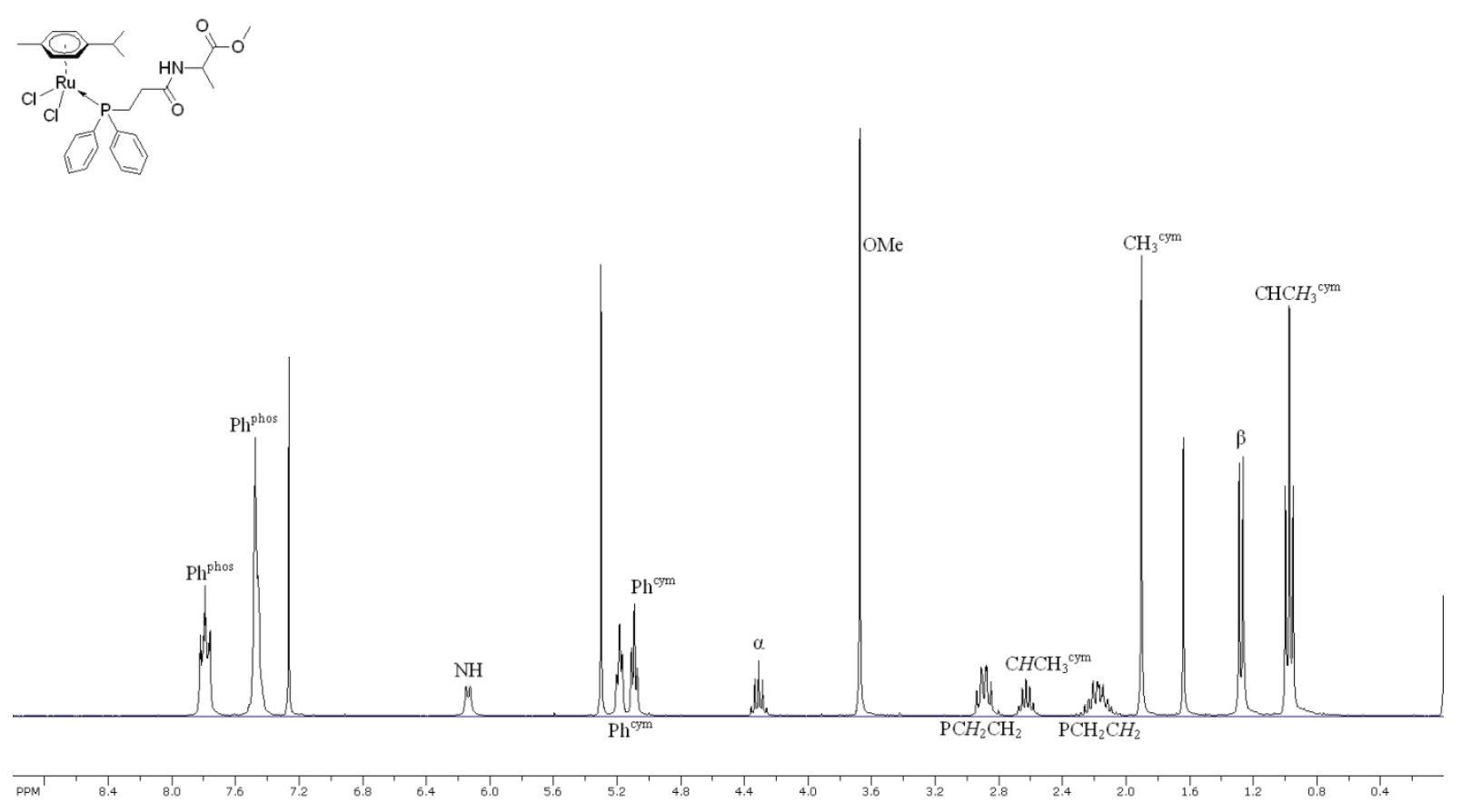

${ }^{13} \mathrm{C}$ NMR $\left(\mathrm{CDCl}_{3}\right)$ spectrum of $\mathbf{1}_{\mathbf{a A}}$.

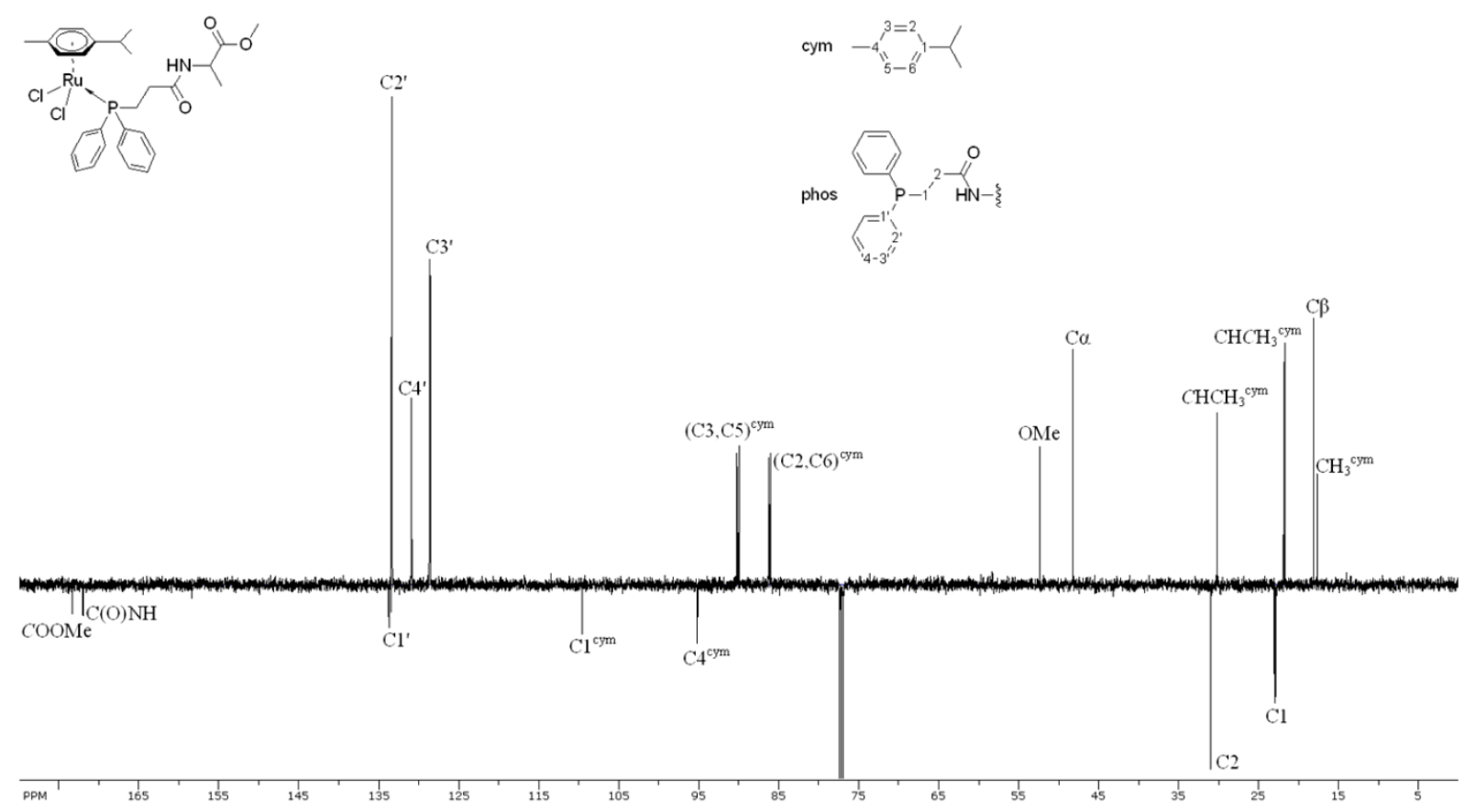


${ }^{1} \mathrm{H}$ NMR $\left(\mathrm{CDCl}_{3}\right)$ spectrum of $\mathbf{2}_{\mathbf{p G}}$.
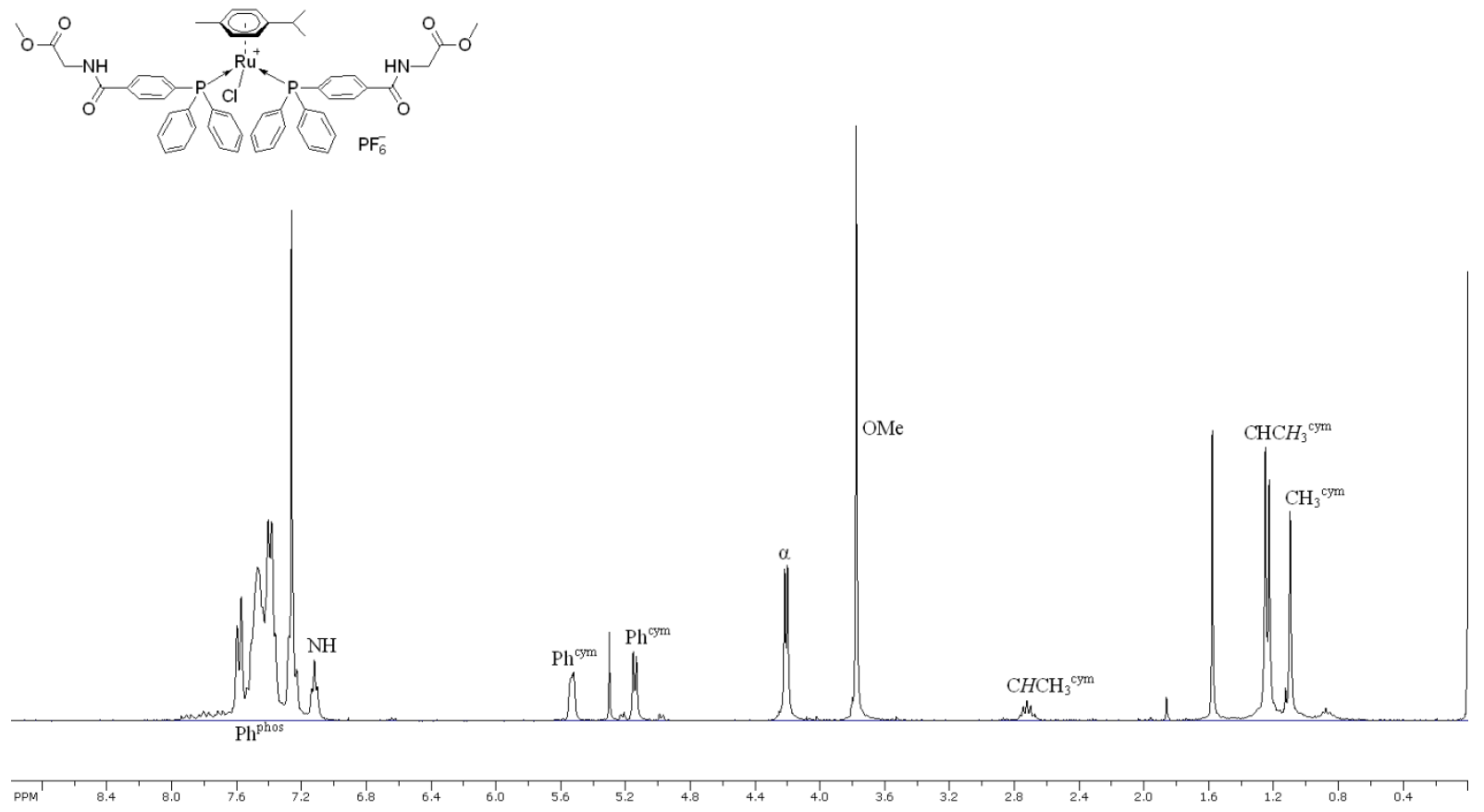

${ }^{13} \mathrm{C} \mathrm{NMR}\left(\mathrm{CDCl}_{3}\right)$ spectrum of $\mathbf{2}_{\mathbf{p G}}$.
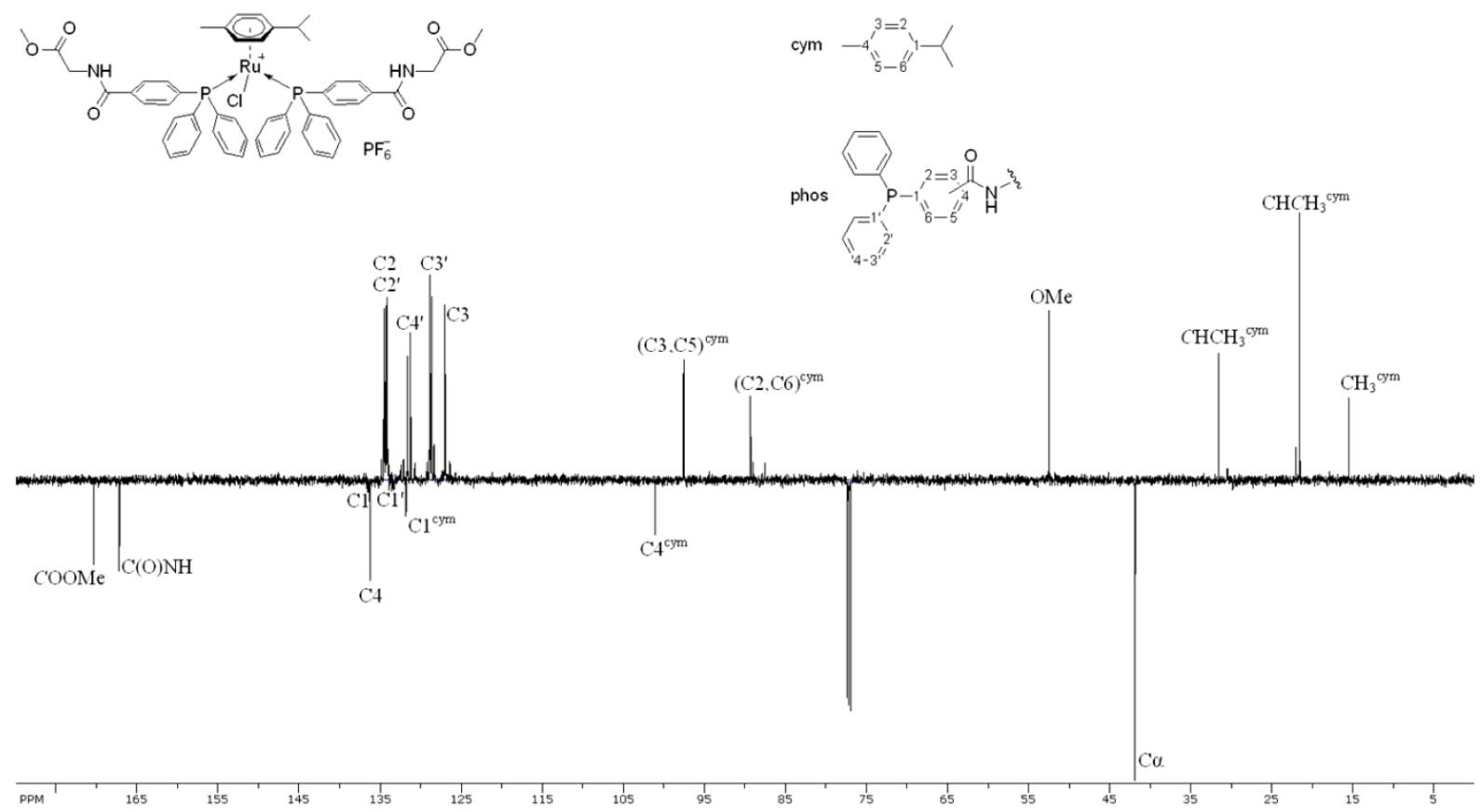
${ }^{1} \mathrm{H}-{ }^{13} \mathrm{C}$ HMQC NMR $\left(\mathrm{CDCl}_{3}\right)$ spectrum of $\mathbf{2}_{\mathbf{p G}}$.

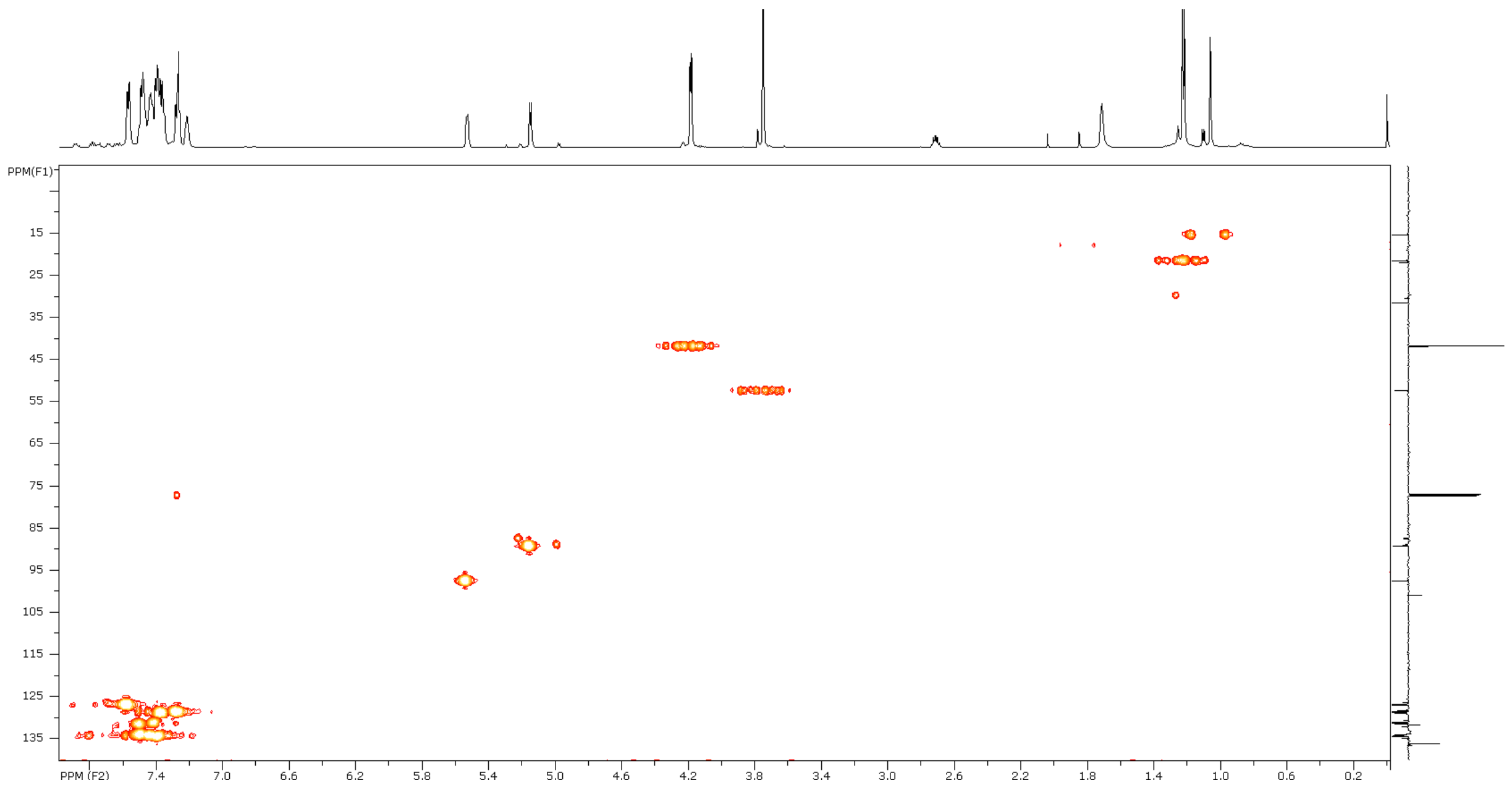


${ }^{1} \mathrm{H}-{ }^{13} \mathrm{C} \mathrm{HMBC}$ NMR $\left(\mathrm{CDCl}_{3}\right)$ spectrum of $\mathbf{2}_{\mathbf{p G}}$.

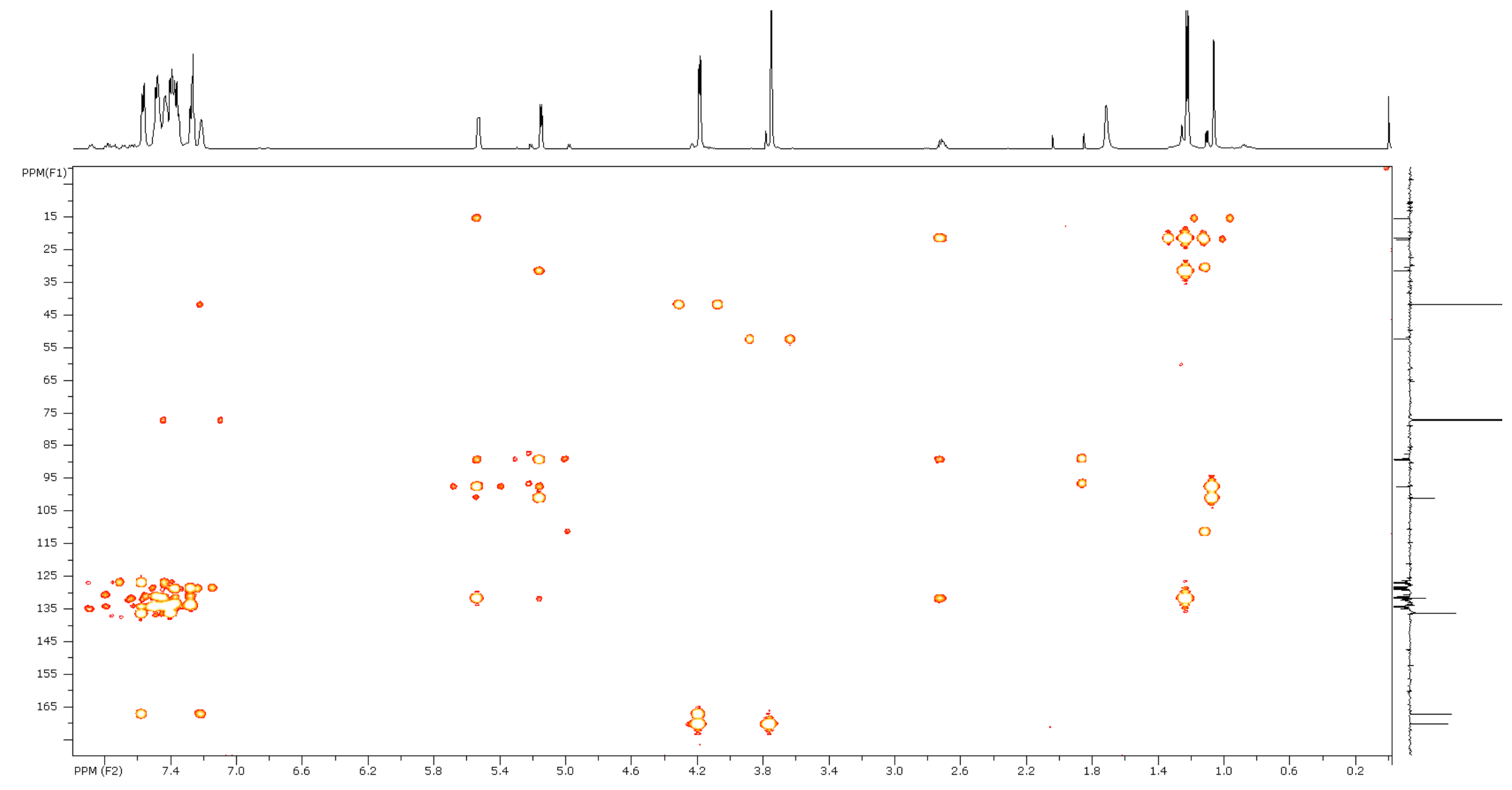


${ }^{1} \mathrm{H}$ NMR $\left(\mathrm{CDCl}_{3}\right)$ spectrum of $\mathbf{2}_{\mathbf{m A}}$.
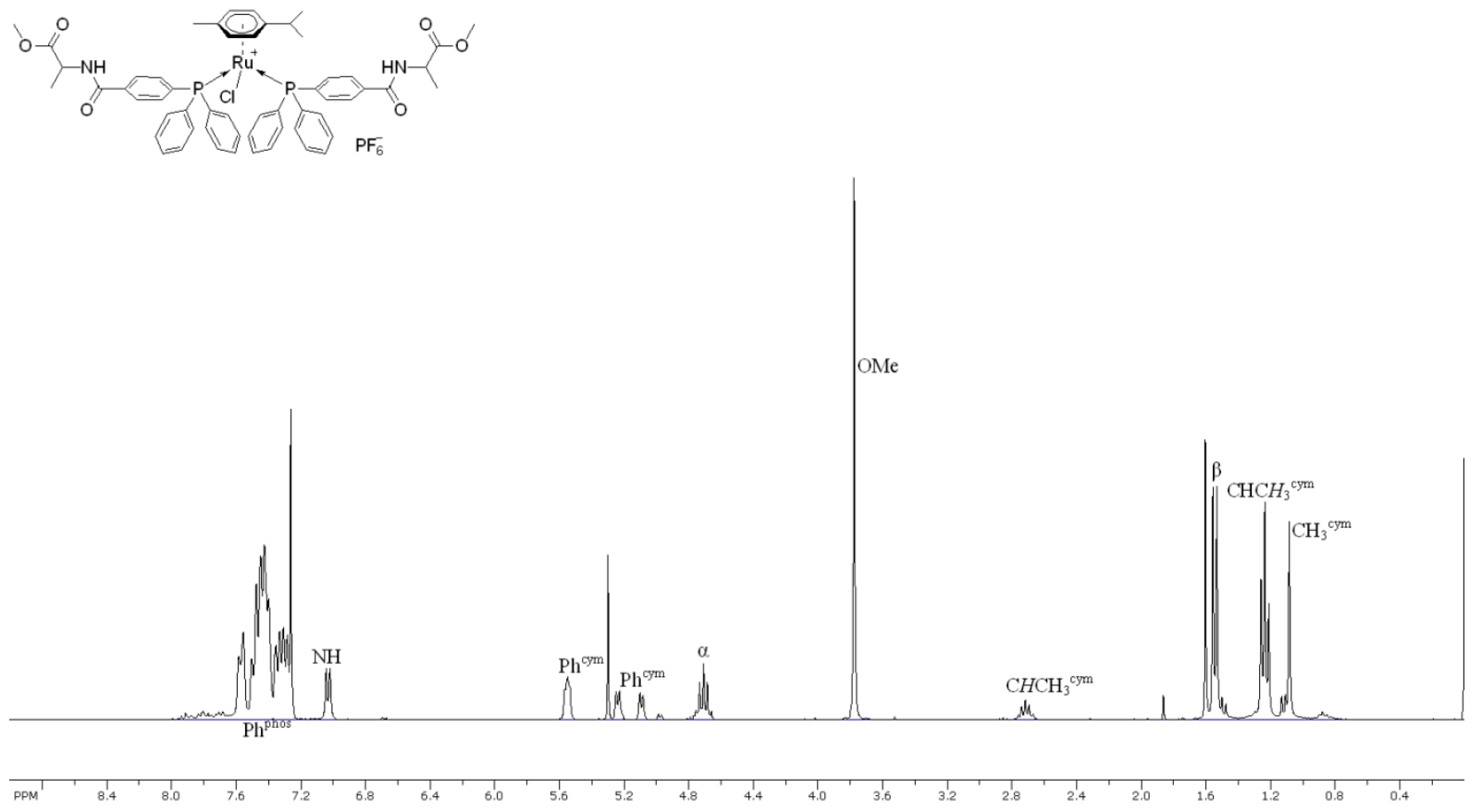

${ }^{13} \mathrm{C}$ NMR $\left(\mathrm{CDCl}_{3}\right)$ spectrum of $\mathbf{2}_{\mathbf{p A}}$.
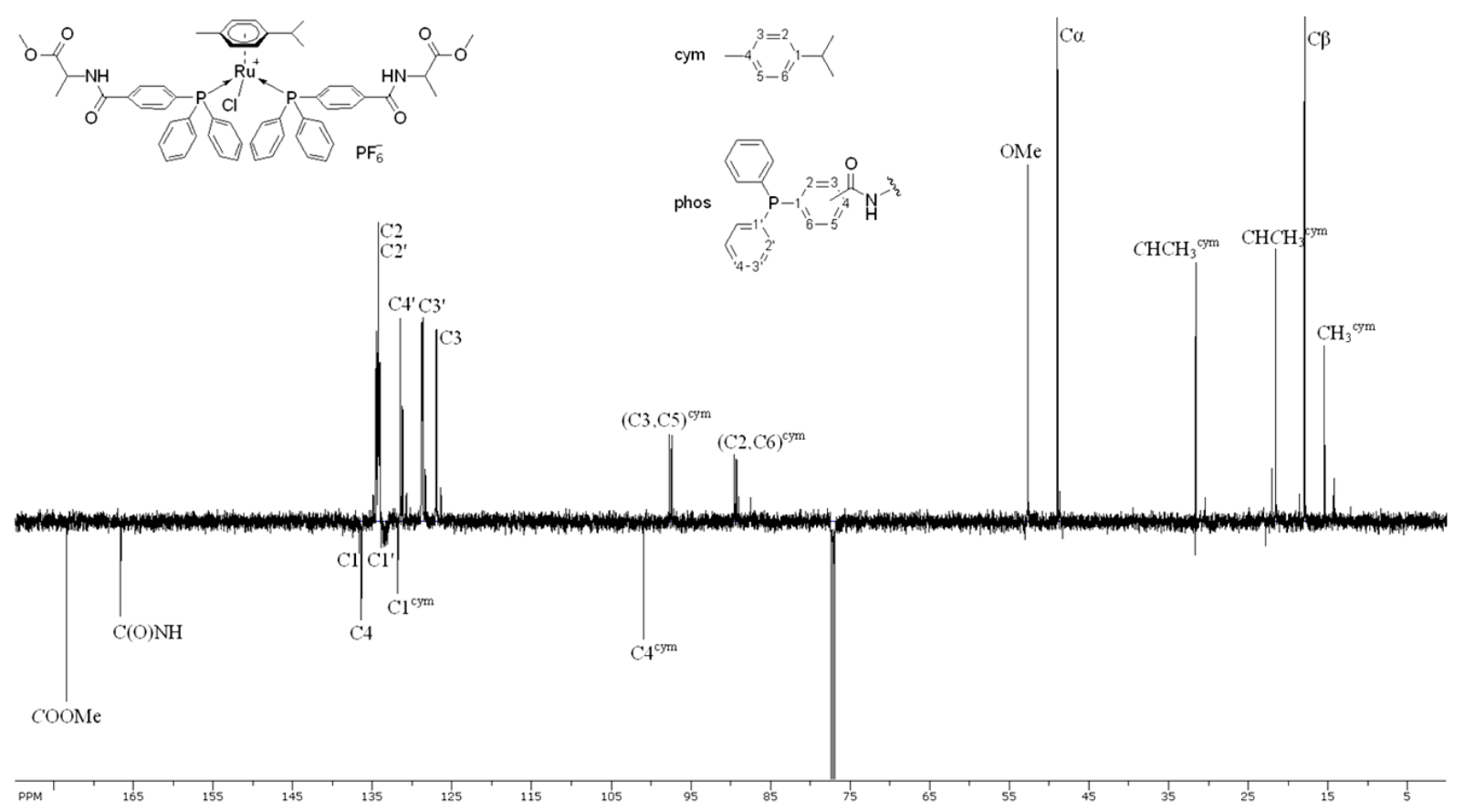
${ }^{1} \mathrm{H} \mathrm{NMR}\left(\mathrm{CDCl}_{3}\right)$ spectrum of $\mathbf{2}_{\mathbf{m G}}$.

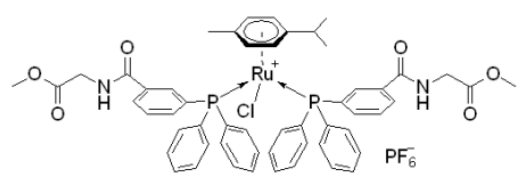

phos
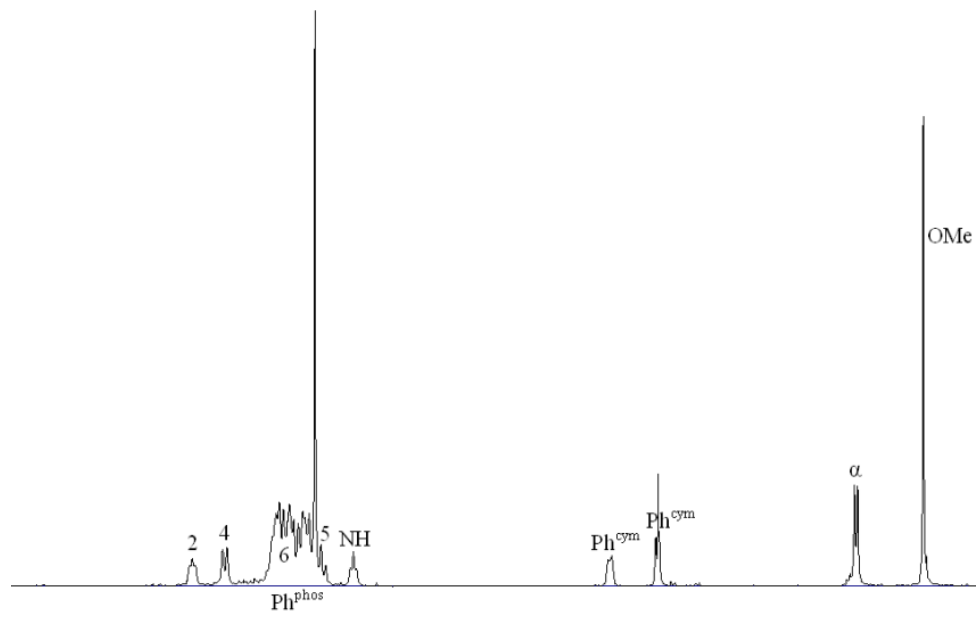

$\mathrm{CHCH}_{3}{ }^{\text {cym }}$

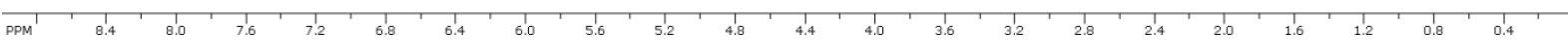

${ }^{13} \mathrm{C} \mathrm{NMR}\left(\mathrm{CDCl}_{3}\right)$ spectrum of $\mathbf{2}_{\mathbf{m G}}$.

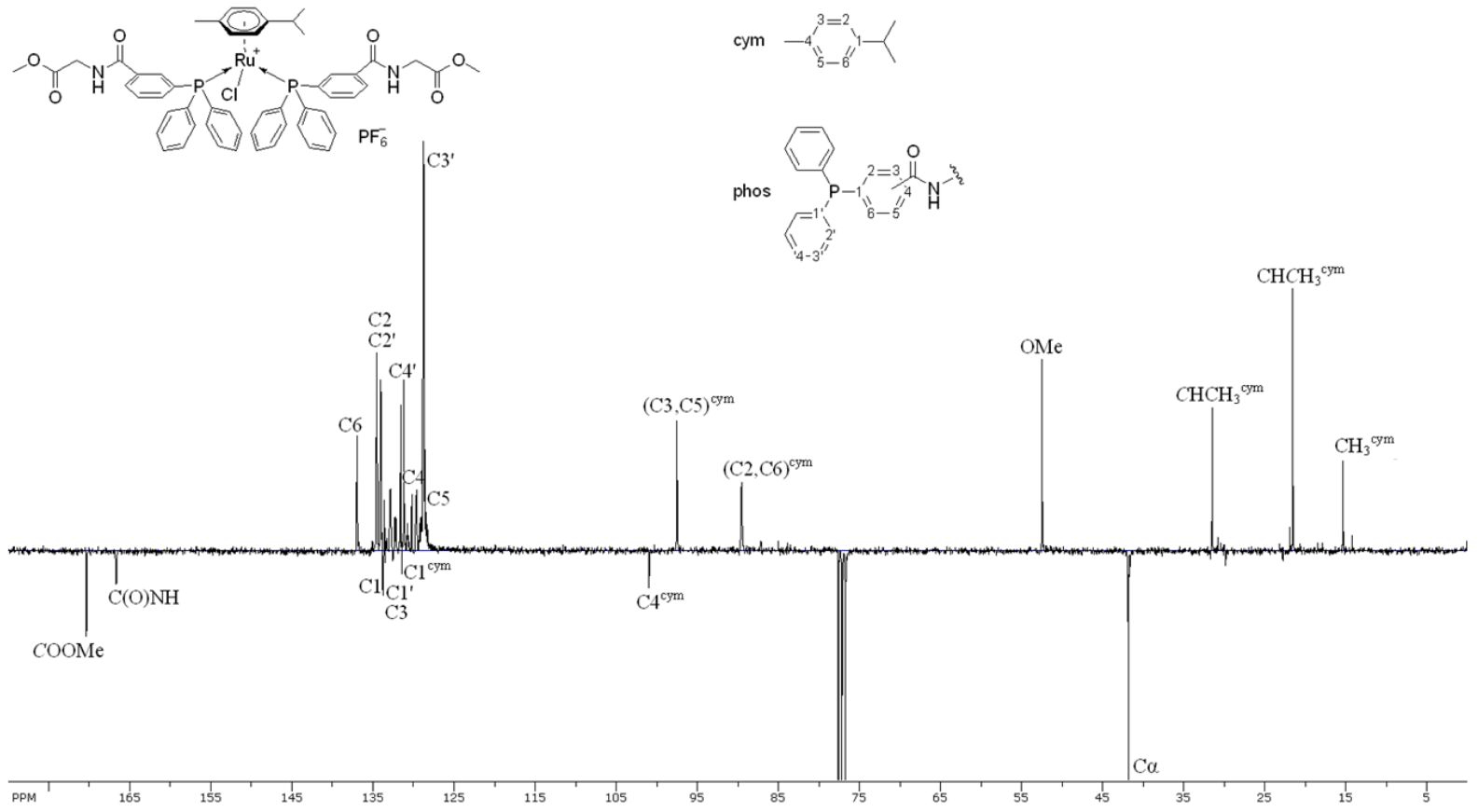


${ }^{1} \mathrm{H}$ NMR $\left(\mathrm{CDCl}_{3}\right)$ spectrum of $\mathbf{2}_{\mathbf{m A}}$.
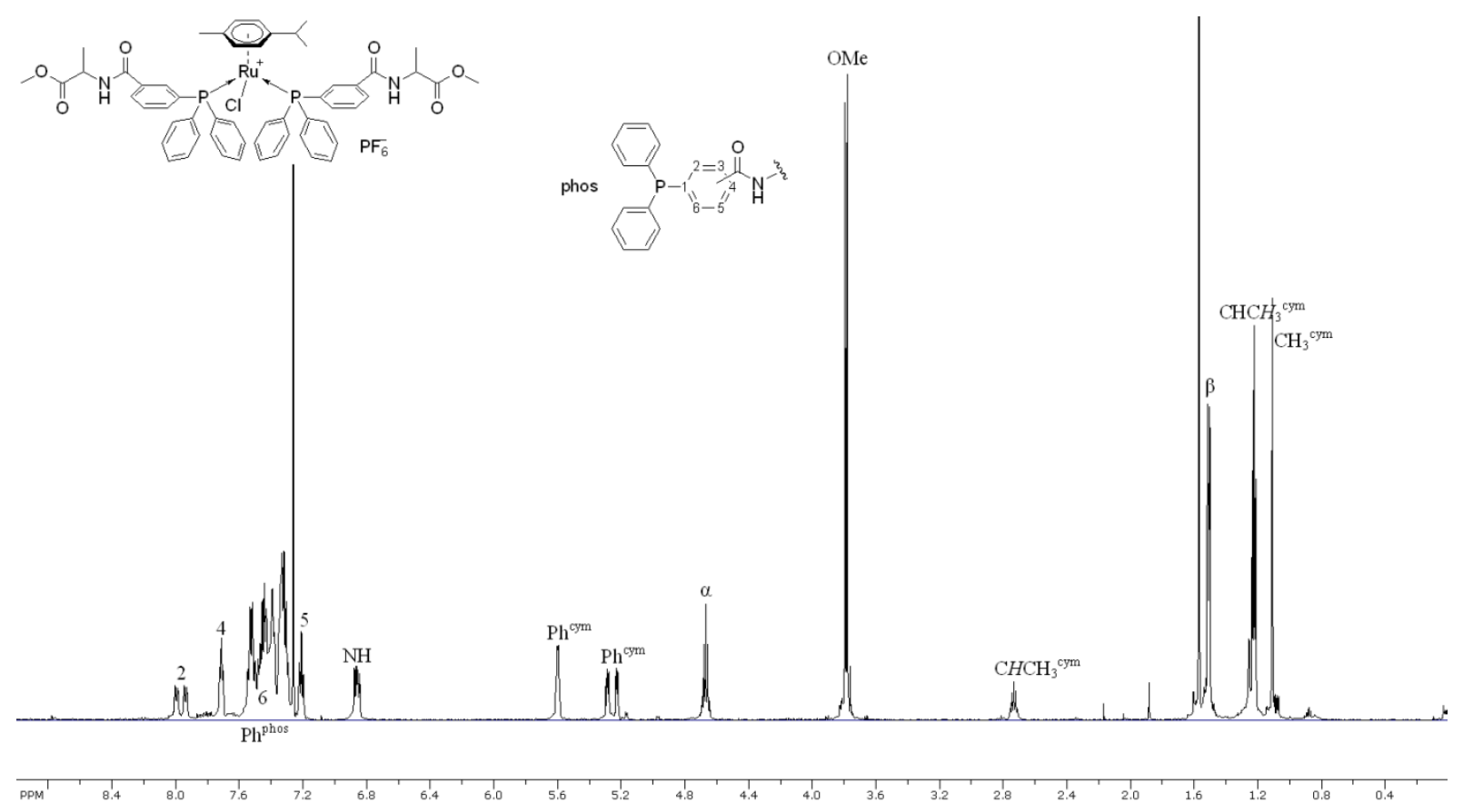

${ }^{13} \mathrm{C} \mathrm{NMR}\left(\mathrm{CDCl}_{3}\right)$ spectrum of $\mathbf{2}_{\mathbf{m A}}$.

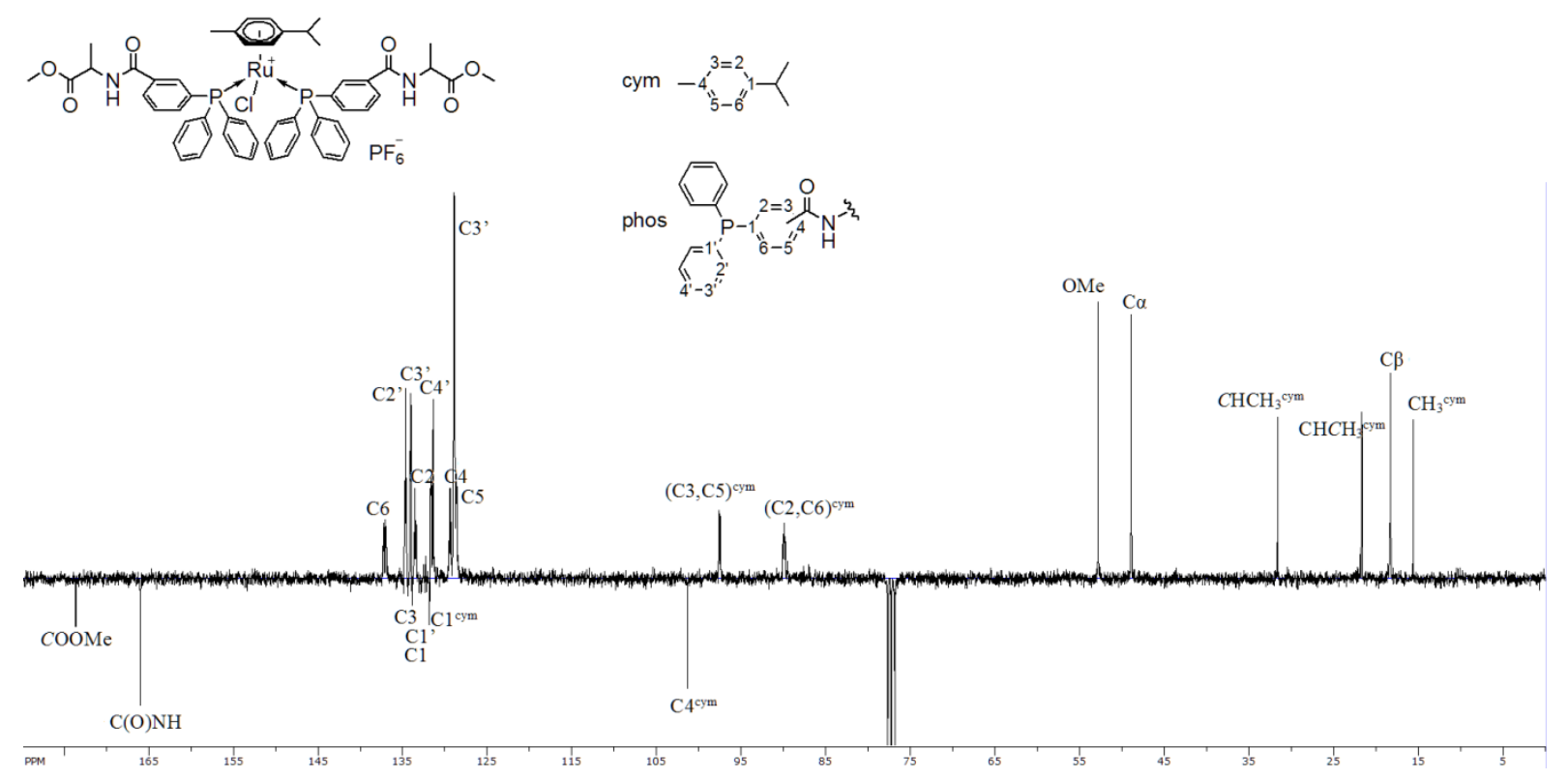




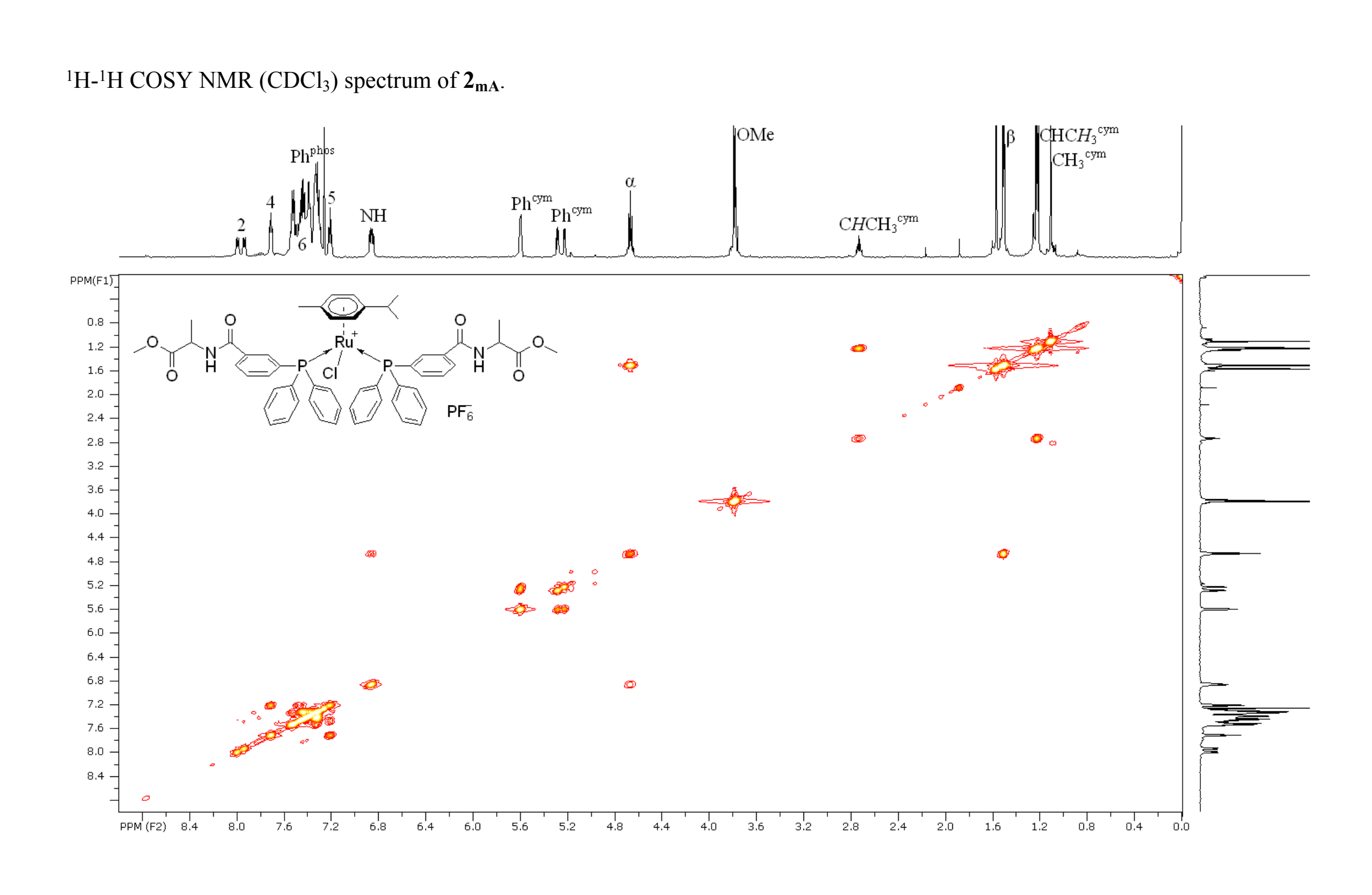


${ }^{1} \mathrm{H}$ NMR $\left(\mathrm{CDCl}_{3}\right)$ spectrum of $\mathbf{2}_{\mathrm{aG}}$.

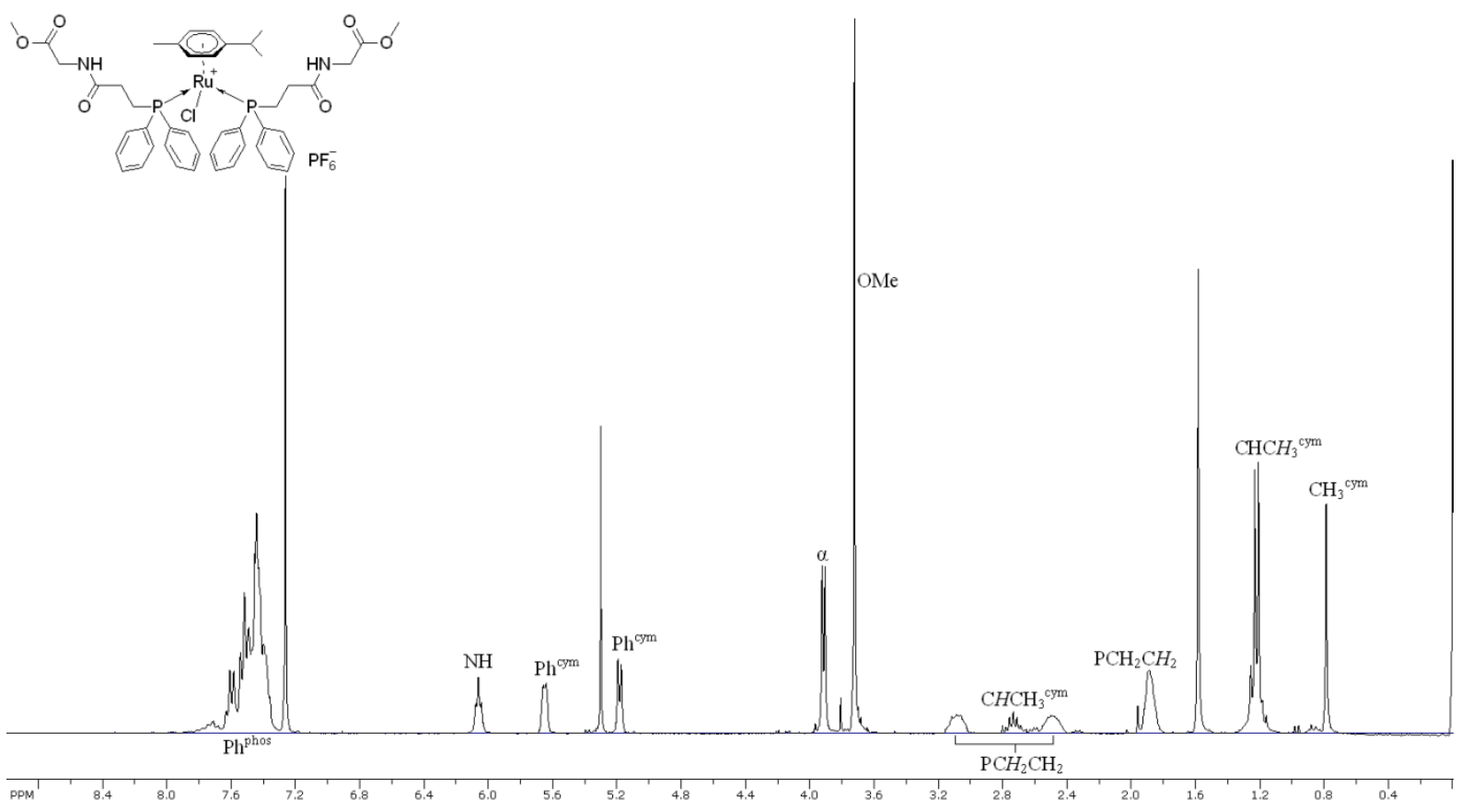

${ }^{13} \mathrm{C} \mathrm{NMR}\left(\mathrm{CDCl}_{3}\right)$ spectrum of $\mathbf{2}_{\mathrm{aG}}$.

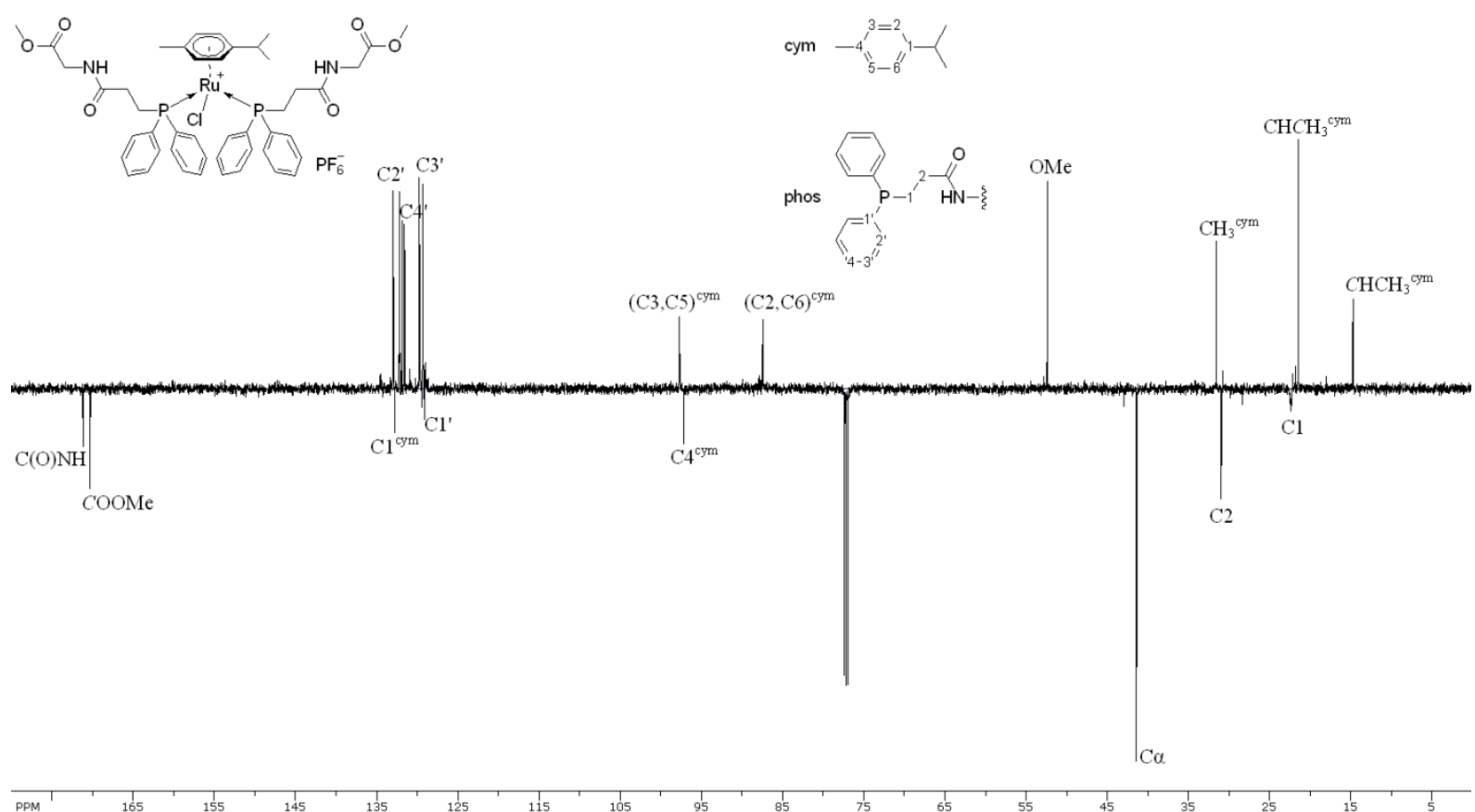


${ }^{1} \mathrm{H}$ NMR $\left(\mathrm{CDCl}_{3}\right)$ spectrum of $\mathbf{2}_{\mathbf{a A}}$.
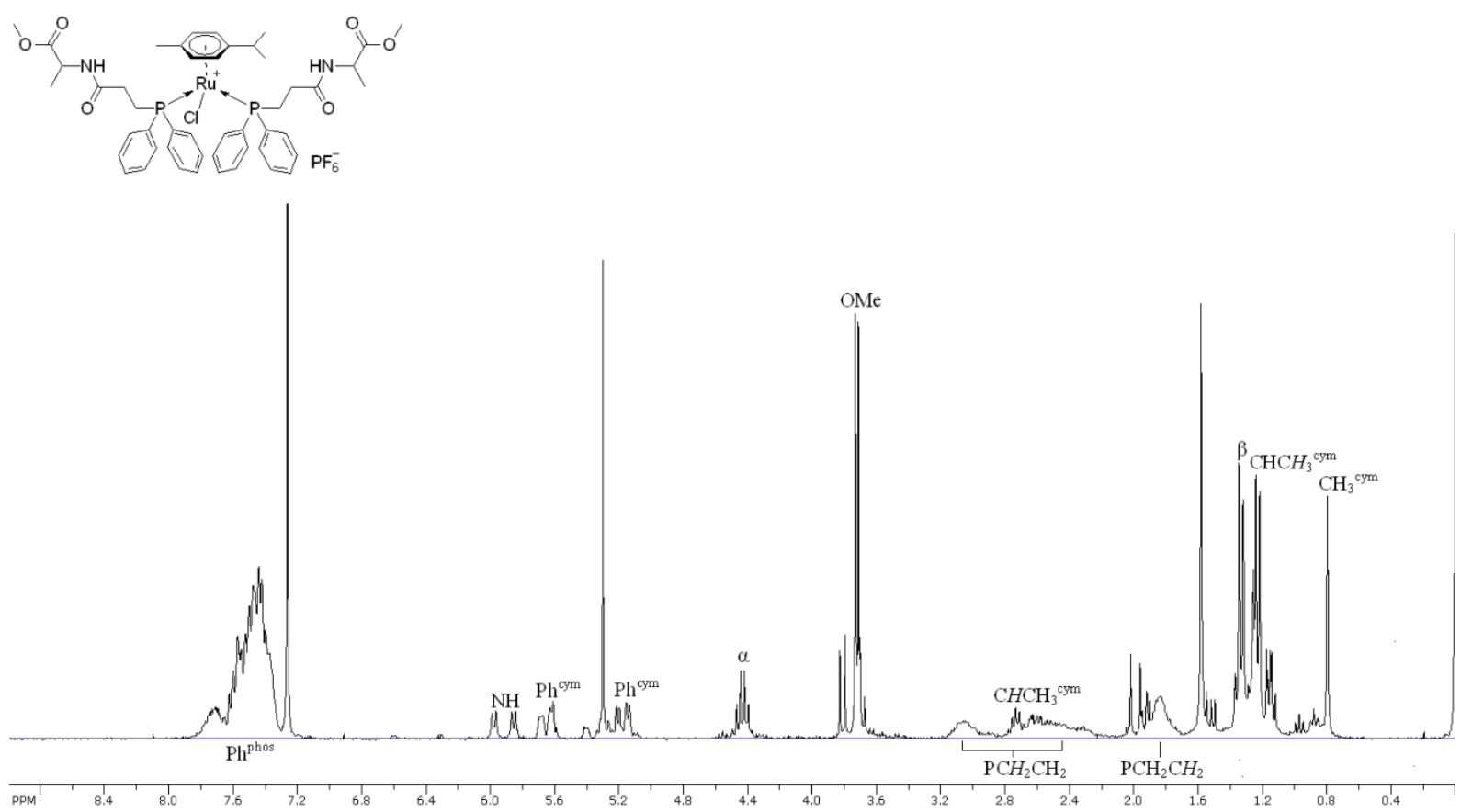

${ }^{13} \mathrm{C}$ NMR $\left(\mathrm{CDCl}_{3}\right)$ spectrum of $\mathbf{2}_{\mathrm{aA}}$.

CD Measurements.

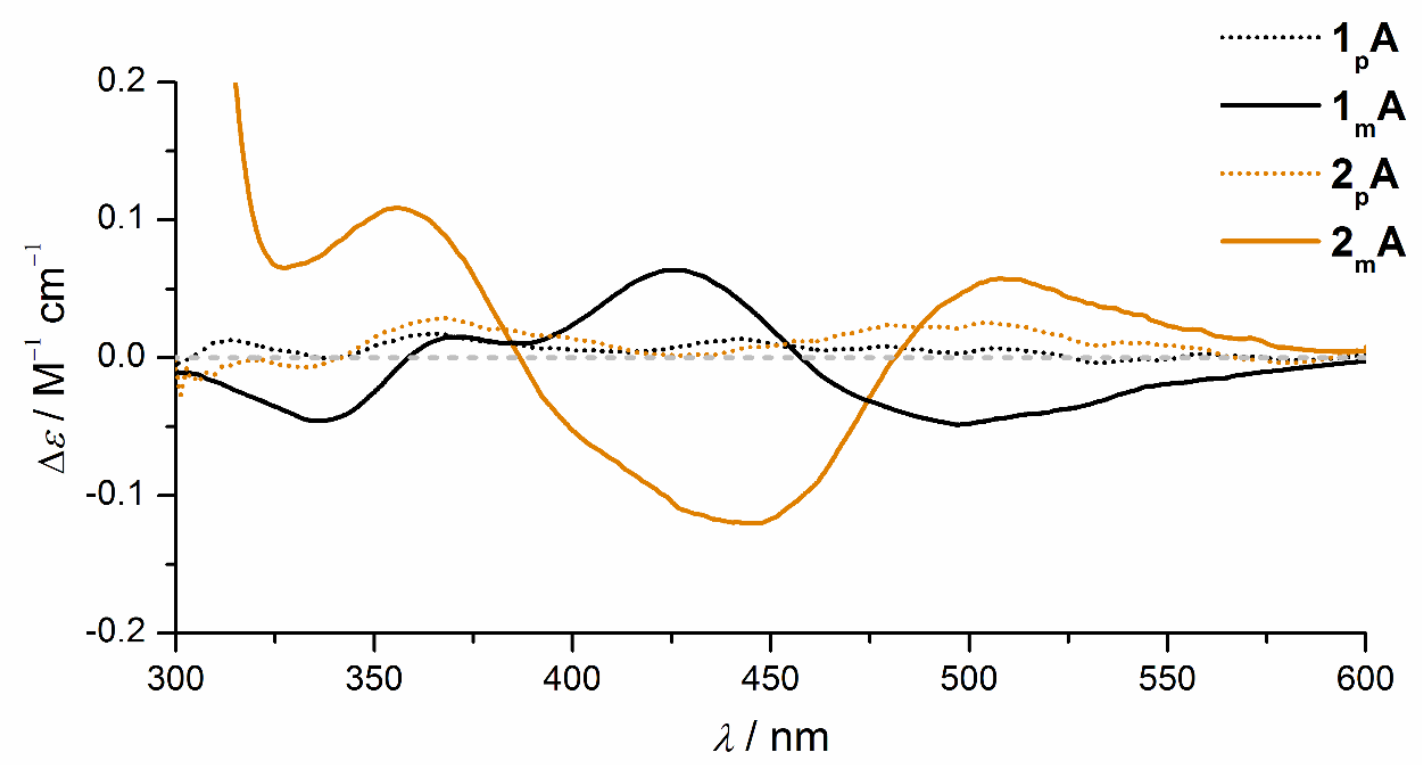

Figure S1. VIS-CD Spectra of Ru complexes 1 and $\mathbf{2}(\mathrm{c}=0.2-0.5 \mathrm{mM})$.



Figure S2. UV-CD Spectra of Ru complexes 1 and $\mathbf{2}(\mathrm{c}=0.03-0.05 \mathrm{mM})$. 


\section{DFT Calculations.}

Table S2. Gibbs energies of various conformers of Ruthenium-aminoacidbioconjugates.

\begin{tabular}{lcccc}
\hline M & $E_{\text {tot }}(\mathbf{M}) /$ a.u. & $G_{\text {corr }} /$ a.u. & $G(\mathbf{M}) /$ a.u. & $G_{\text {rel }}(\mathbf{M}) / \mathrm{kJ} \mathrm{mol}^{-1, \mathrm{a}}$ \\
\hline 2mGs-I & -3809.56110 & 0.83383 & -3808.72727 & 0.0 \\
2mGs-Ib & -3809.55194 & 0.82894 & -3808.72300 & 11.2 \\
2mGs-II & -3809.55479 & 0.83268 & -3808.72211 & 13.6 \\
2mGa-I & -3809.55310 & 0.83005 & -3808.72305 & 11.1 \\
2mGa-II & -3809.54194 & 0.82745 & -3808.71450 & 33.5 \\
2pG-I & -3809.54564 & 0.82858 & -3808.71705 & 26.8 \\
2pG-II & -3809.54379 & 0.82904 & -3808.71475 & 32.9
\end{tabular}

\section{M-helicity}

2mAs-Im

$$
-3888.17585
$$

0.89033

$-3887.28553$

20.1

2mAs-Ibm

$-3888.17146$

0.88424

$-3887.28722$

15.7

2mAs-IIm

$-3888.16412$

0.88387

$-3887.28026$

33.9

2mAa-Im

$-3888.17289$

0.88662

$-3887.28627$

18.2

2mAa-IIm

$-3888.16230$

0.88290

$-3887.27941$

36.2

2pA-Im

$-3888.16088$

0.88408

$-3887.27680$

43.0

2pA-IIm

$-3888.15647$

0.88207

$-3887.27440$

49.3

\section{P-helicity}

\begin{tabular}{llllr}
\hline 2mAs-Ip & -3888.17910 & 0.88591 & -3887.29319 & 0.0 \\
2mAs-IIp & -3888.17447 & 0.88443 & -3887.29004 & 8.3 \\
2mAa-Ip & -3888.16882 & 0.88419 & -3887.28463 & 22.5 \\
2mAa-IIp & -3888.16126 & 0.88141 & -3887.27986 & 35.0 \\
2pA-Ip & -3888.16415 & 0.88009 & -3887.28406 & 23.9 \\
2pA-IIp & -3888.16401 & 0.88419 & -3887.27982 & 35.1 \\
\hline
\end{tabular}

a) Relative Gibbs energies $\left(G_{\text {rel }}\right)$ were calculated using the most stable glycine (2mGs-I) or alanine (2mAs-I) complexes according to eq.: $G_{\text {rel }}(\mathbf{M})=G(\mathbf{M})-G_{\text {tot }}(2 \mathbf{m X s}-\mathrm{I})$ where $\mathrm{X}=\mathrm{G}$ or A. In the case of alanine derivative, P-helical derivative was the most stable one and this structure was used as the reference. 
Biomacromolecular target evaluation.

Thermal melting experiments.

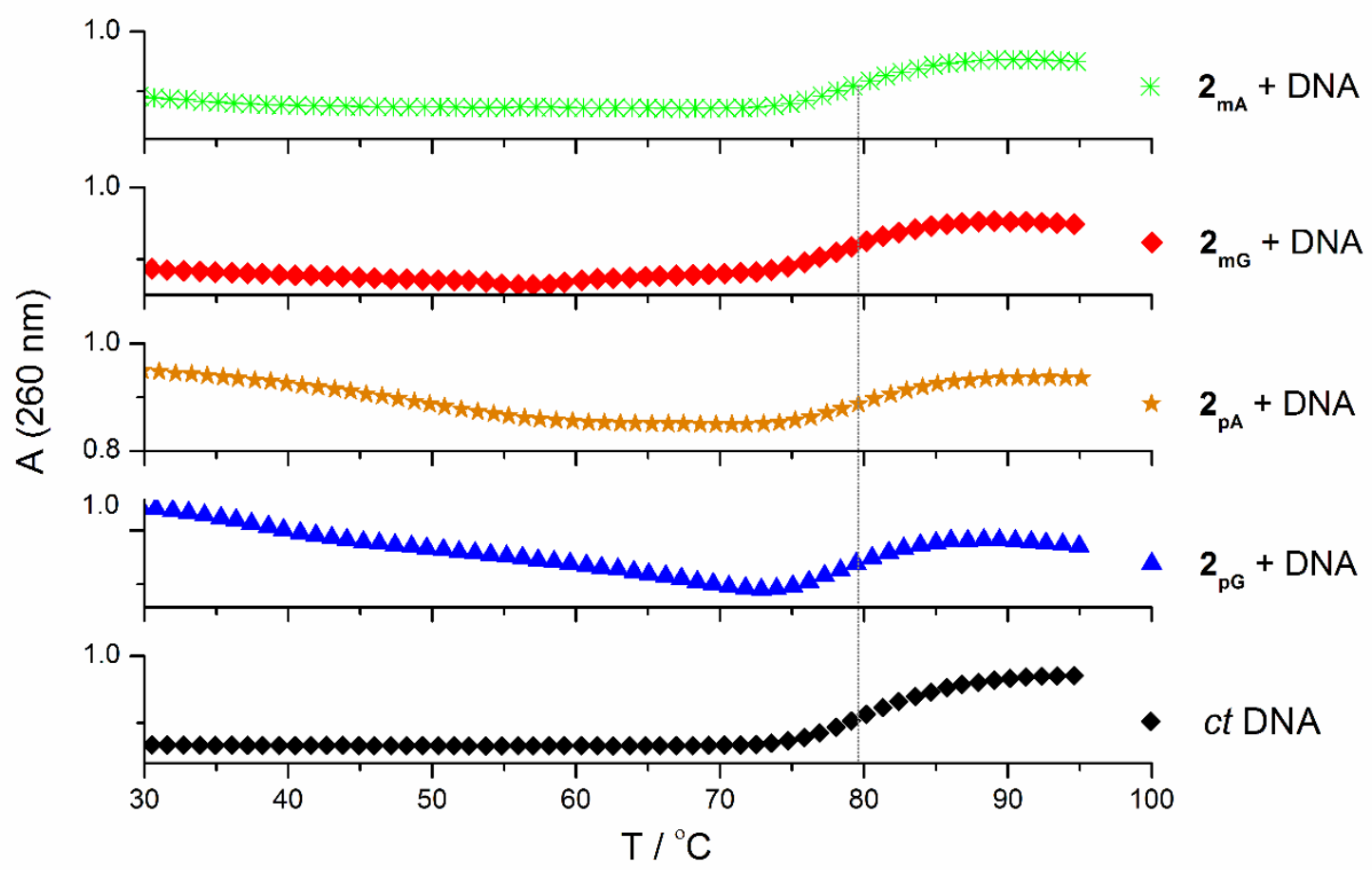

Figure S3. Melting curves of $c t$-DNA upon addition of examined ruthenium complexes (c (ctDNA $)=2 \times 10^{-5} \mathrm{M}$; ratio $r$ [compound $\left.] /[\mathrm{DNA}]=0.3\right)$ at $\mathrm{pH}=7.0$, sodium cacodylate buffer, $I$ $\left.=0.05 \mathrm{~mol} \mathrm{dm}^{-3}\right)$. 


\section{CD titrations.}
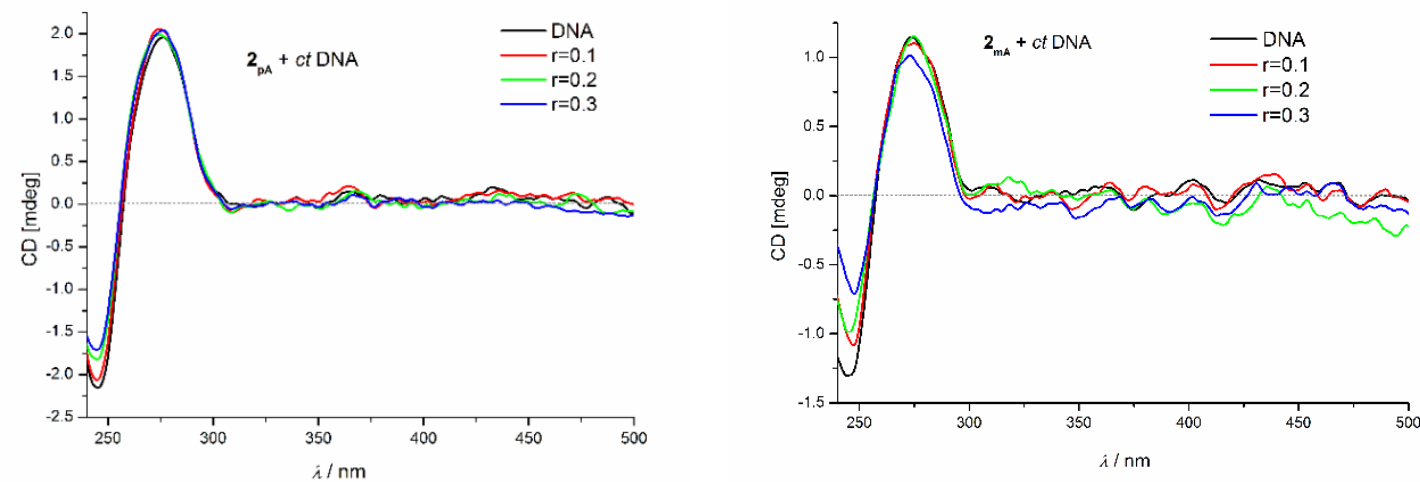

Figure S4. CD spectrum of of $c t$-DNA upon addition of $2 \mathrm{pG}$ (left) and $2 \mathrm{pA}$ (right) at different molar ratios $\mathrm{r}=$ [compound] / [polynucleotide]; $\left(\mathrm{c}(c t\right.$-DNA $)=2 \times 10^{-5} \mathrm{M} ; \mathrm{pH}=7.0$, sodium cacodylate buffer, $I=0.05 \mathrm{~mol} \mathrm{\textrm {dm } ^ { - 3 }}$ ).
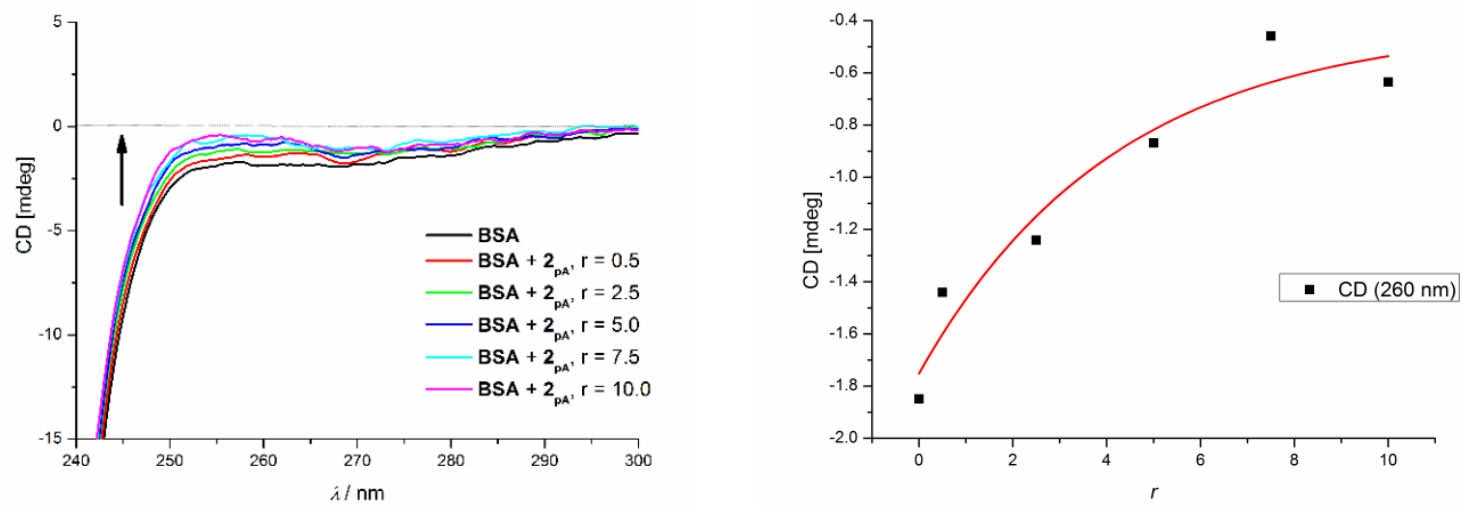

Figure S5. Left: Changes in the CD spectrum of BSA $\left(c=2 \times 10^{-6} \mathrm{~mol} \mathrm{dm}^{-3}\right)$ upon addition of $\mathbf{2}_{\mathbf{p A}}$ at different molar ratios $r=$ [ruthenium complex] / [protein], $\mathrm{pH}=7.0$, Na-cacodylate buffer, $I=0.05 \mathrm{~mol} \mathrm{dm}^{-3}$. Right: Dependence of CD spectra at $260 \mathrm{~nm}$ on ratio $r=$ [ruthenium complex]/[protein]. 


\section{Fluorimetric titrations.}

Fluorescence titration data were fitted by Specfit program* to 1:1 stoichiometry of complex of BSA-ruthenium complex.
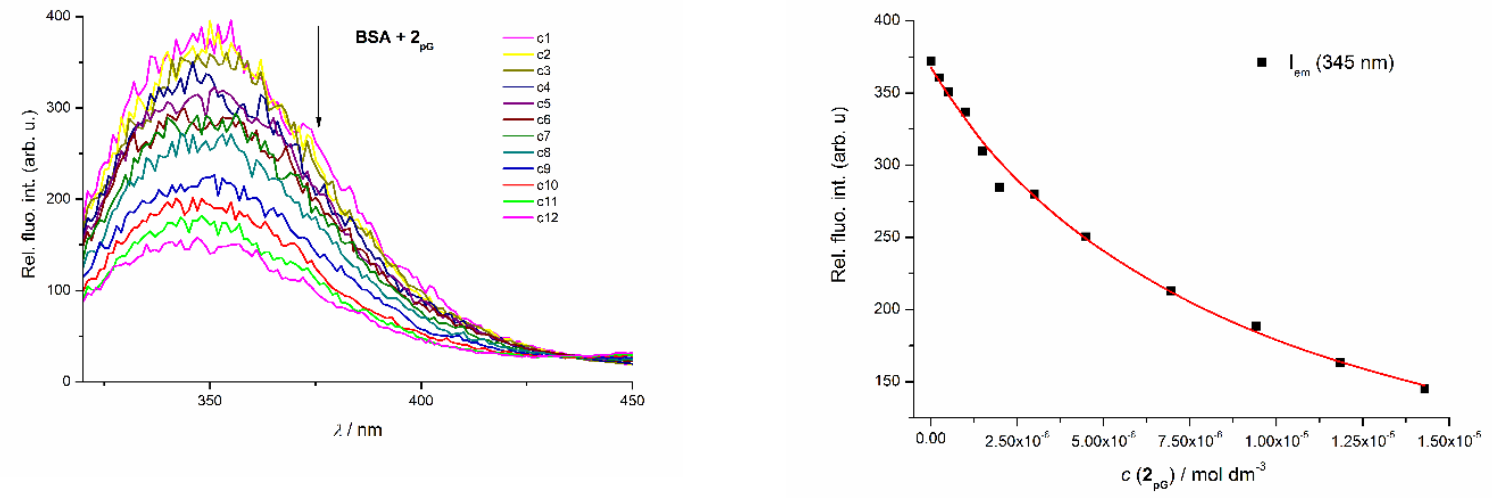

Figure S6. Left: Fluorimetric titration of BSA with, $\lambda_{\text {exc }}=300 \mathrm{~nm}, \mathrm{c}(\mathrm{BSA})=5 \times 10^{-7} \mathrm{~mol}$ $\mathrm{dm}^{-3}$, c $\left(\mathbf{2}_{\mathrm{pG}}\right)=2.5 \times 10^{-7} \mathrm{~mol} \mathrm{dm} \mathrm{dm}^{-3}-1.4 \times 10^{-5} \mathrm{~mol} \mathrm{dm}{ }^{-3}$, Right: Experimental $(\bullet)$ and calculated (-) (SPECFIT, Table 3 in the manuscript) fluorescence intensities of BSA at $\lambda_{\mathrm{em}}=$ $345 \mathrm{~nm}$ upon addition of $\mathbf{2}_{\mathbf{p G}}\left(\mathrm{pH}=7.0, \mathrm{Na}\right.$-cacodylate buffer, $\left.\mathrm{I}=0.05 \mathrm{~mol} \mathrm{dm}^{-3}\right)$.
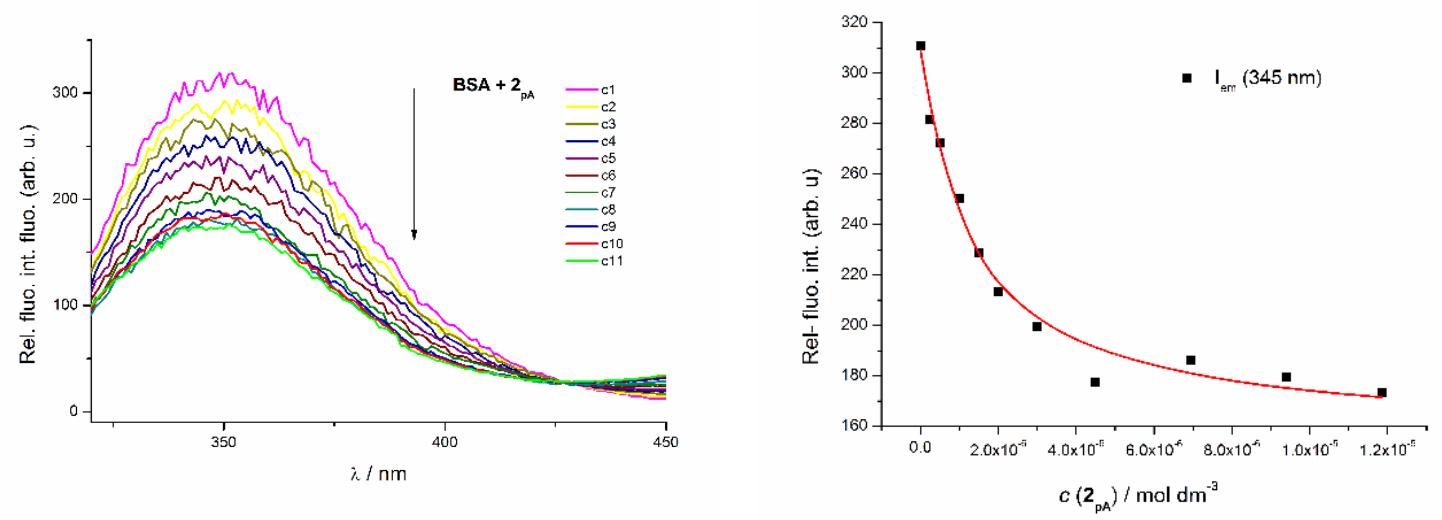

Figure S7. Left: Fluorimetric titration of BSA with, $\lambda_{\text {exc }}=300 \mathrm{~nm}, \mathrm{c}(\mathrm{BSA})=5 \times 10^{-7} \mathrm{~mol}$ $\mathrm{dm}^{-3}, \mathrm{c}\left(\mathbf{2}_{\mathrm{pA}}\right)=2.5 \times 10^{-7} \mathrm{~mol} \mathrm{dm}^{-3}-1.2 \times 10^{-5} \mathrm{~mol} \mathrm{dm}^{-3}$, Right: Experimental $(\bullet)$ and calculated ( - ) (SPECFIT, Table 3 ) fluorescence intensities of BSA at $\lambda_{\mathrm{em}}=345 \mathrm{~nm}$ upon addition of $\mathbf{2}_{\mathbf{p A}}\left(\mathrm{pH}=7.0\right.$, Na-cacodylate buffer, $\left.\mathrm{I}=0.05 \mathrm{~mol} \mathrm{dm}^{-3}\right)$.

* Reference: Gampp H, Maeder M, Meyer CJ, Zuberbuhler AD (1985). Calculation of Equilibrium-Constants from Multiwavelength Spectroscopic Data .2. Specfit - 2 UserFriendly Programs in Basic and Standard Fortran-77. Talanta32: 257-264. 

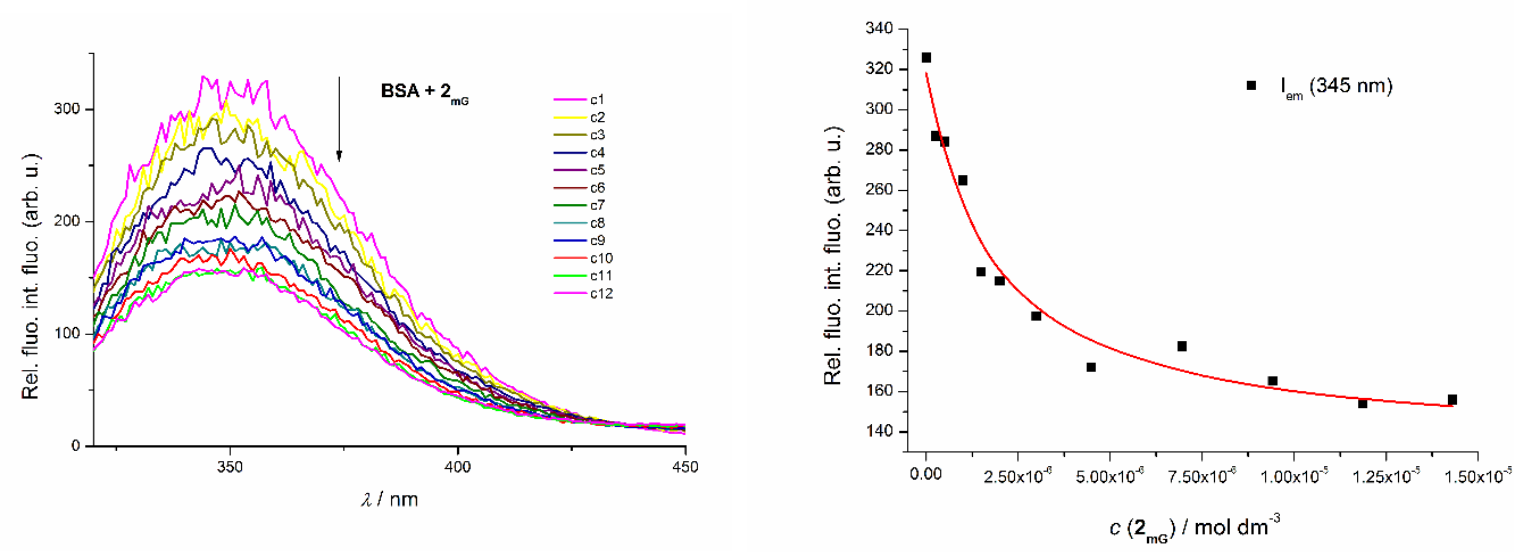

Figure S8. Left: Fluorimetric titration of BSA with, $\lambda_{\mathrm{exc}}=300 \mathrm{~nm}, \mathrm{c}(\mathrm{BSA})=5 \times 10^{-7} \mathrm{~mol}$ $\mathrm{dm}^{-3}$, c $\left(\mathbf{2}_{\mathrm{mG}}\right)=2.5 \times 10^{-7} \mathrm{~mol} \mathrm{dm}^{-3}-1.2 \times 10^{-5} \mathrm{~mol} \mathrm{dm}{ }^{-3}$, Right: Experimental $(\bullet)$ and calculated (-) (SPECFIT, Table 3) fluorescence intensities of BSA at $\lambda_{\mathrm{em}}=345 \mathrm{~nm}$ upon addition of $2_{\mathbf{m G}}\left(\mathrm{pH}=7.0\right.$, Na-cacodylate buffer, $\left.\mathrm{I}=0.05 \mathrm{~mol} \mathrm{dm}^{-3}\right)$.
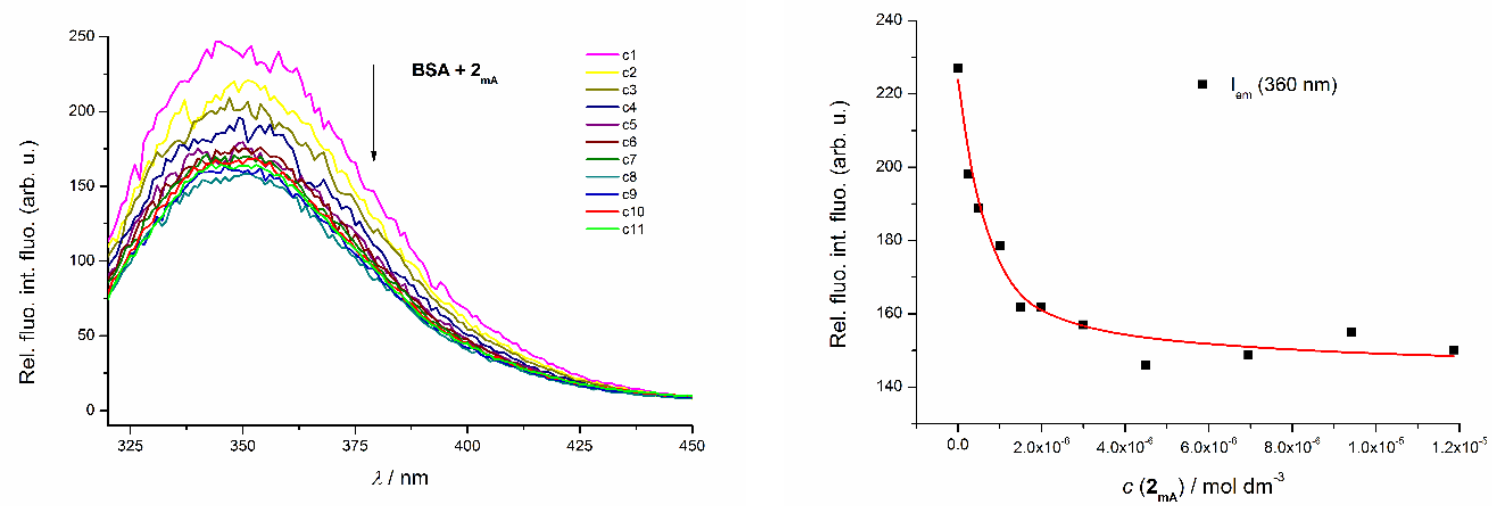

Figure S9. Left: Fluorimetric titration of BSA with, $\lambda_{\mathrm{exc}}=300 \mathrm{~nm}, \mathrm{c}(\mathrm{BSA})=5 \times 10^{-7} \mathrm{~mol}$ $\mathrm{dm}^{-3}, \mathrm{c}\left(\mathbf{2}_{\mathrm{mA}}\right)=2.5 \times 10^{-7} \mathrm{~mol} \mathrm{dm}^{-3}-1.2 \times 10^{-5} \mathrm{~mol} \mathrm{dm}^{-3}$, Right: Experimental $(\bullet)$ and calculated (-) (SPECFIT, Table 3) fluorescence intensities of BSA at $\lambda_{\text {em }}=345 \mathrm{~nm}$ upon addition of $2_{\mathrm{mA}}\left(\mathrm{pH}=7.0\right.$, Na-cacodylate buffer, $\left.\mathrm{I}=0.05 \mathrm{~mol} \mathrm{dm}^{-3}\right)$. 


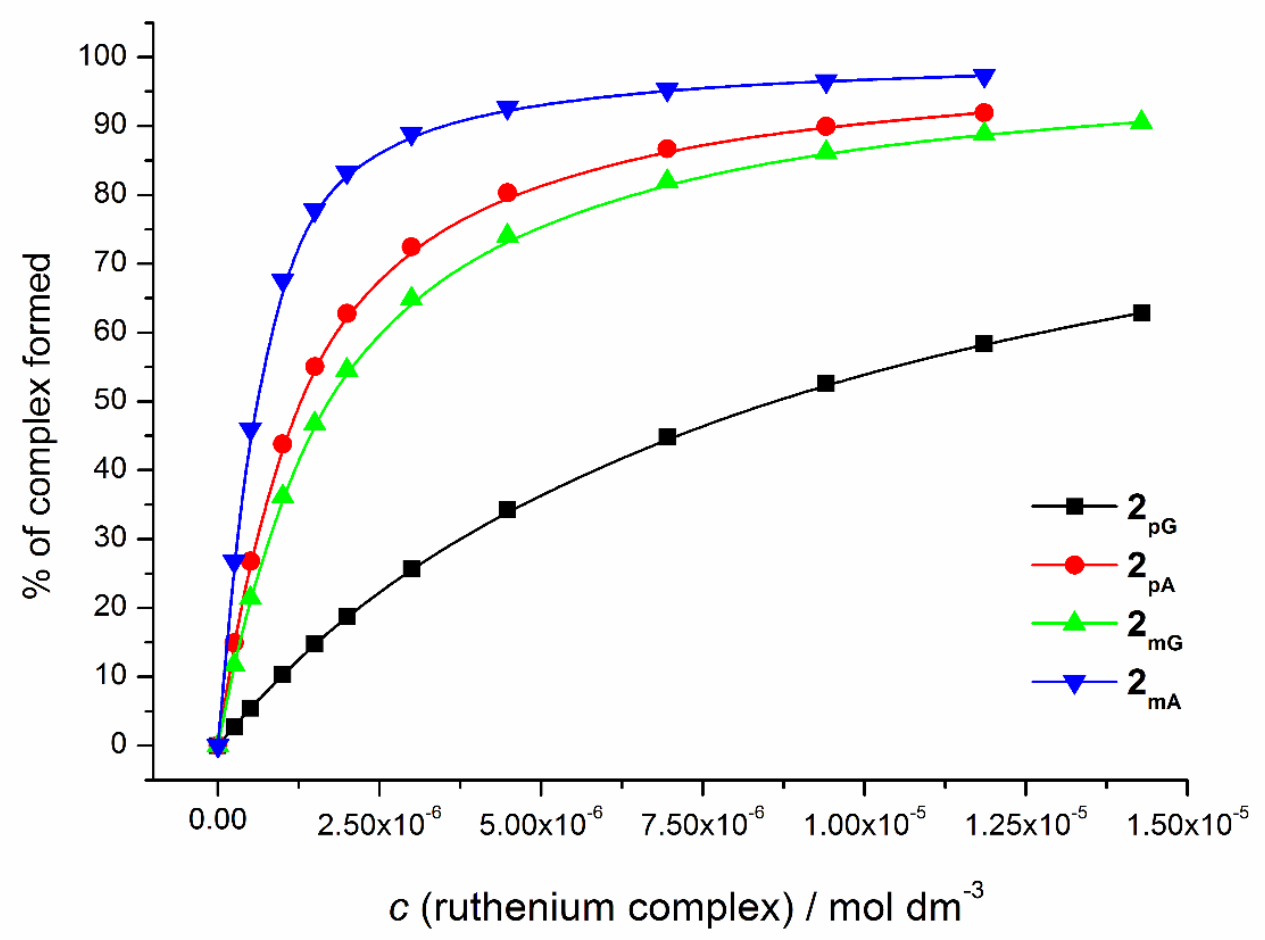

Figure S10. Percentage of formed ruthenium compound / BSA complex calculated by Specfit. 


\section{Biological evaluation.}

Table S3. The cytotoxic activity of compounds towards HeLa cells $\left(\mathrm{IC}_{50} \mu \mathrm{M} \pm \mathrm{SD}\right) . \mathrm{IC}_{50}$ values were calculated upon $72 \mathrm{~h}$ incubation with the compounds. The cytotoxicity was measured by MTT assay. Cp. Compound.

\begin{tabular}{|c|c|}
\hline $\mathbf{C p .}$ & $\mathbf{I C}_{\mathbf{5 0}} \pm \mathbf{S D}(\boldsymbol{\mu M})$ \\
\hline $\mathbf{L}_{\mathbf{p G}}$ & $>33.3$ \\
\hline $\mathbf{L}_{\mathbf{p A}}$ & $>33.3$ \\
\hline $\mathbf{L}_{\mathbf{m G}}$ & $>33.3$ \\
\hline $\mathbf{L}_{\mathbf{a G}}$ & $>33.3$ \\
\hline $\mathbf{L}_{\mathbf{a A}}$ & $>33.3$ \\
\hline $\mathbf{1}_{\mathbf{p G}}$ & $>33.3$ \\
\hline $\mathbf{1}_{\mathbf{p A}}$ & $>33.3$ \\
\hline $\mathbf{1}_{\mathbf{m G}}$ & $>33.3$ \\
\hline $\mathbf{1}_{\mathbf{m A}}$ & $>33.3$ \\
\hline $\mathbf{1}_{\mathbf{a G}}$ & $>33.3$ \\
\hline $\mathbf{1}_{\mathbf{a A}}$ & $>33.3$ \\
\hline $\mathbf{2}_{\mathbf{p G}}$ & $30 \pm 3.2$ \\
\hline $\mathbf{2}_{\mathbf{p A}}$ & $15 \pm 3.7$ \\
\hline $\mathbf{2}_{\mathbf{m G}}$ & $16 \pm 2.5$ \\
\hline $\mathbf{2}_{\mathbf{m A}}$ & $5 \pm 2.7$ \\
\hline$\left.\{\mathbf{( p C y m}) \mathbf{R u C l}_{\mathbf{2}} \mathbf{C l}_{\mathbf{2}}\right]$ & $>33.3$ \\
\hline
\end{tabular}



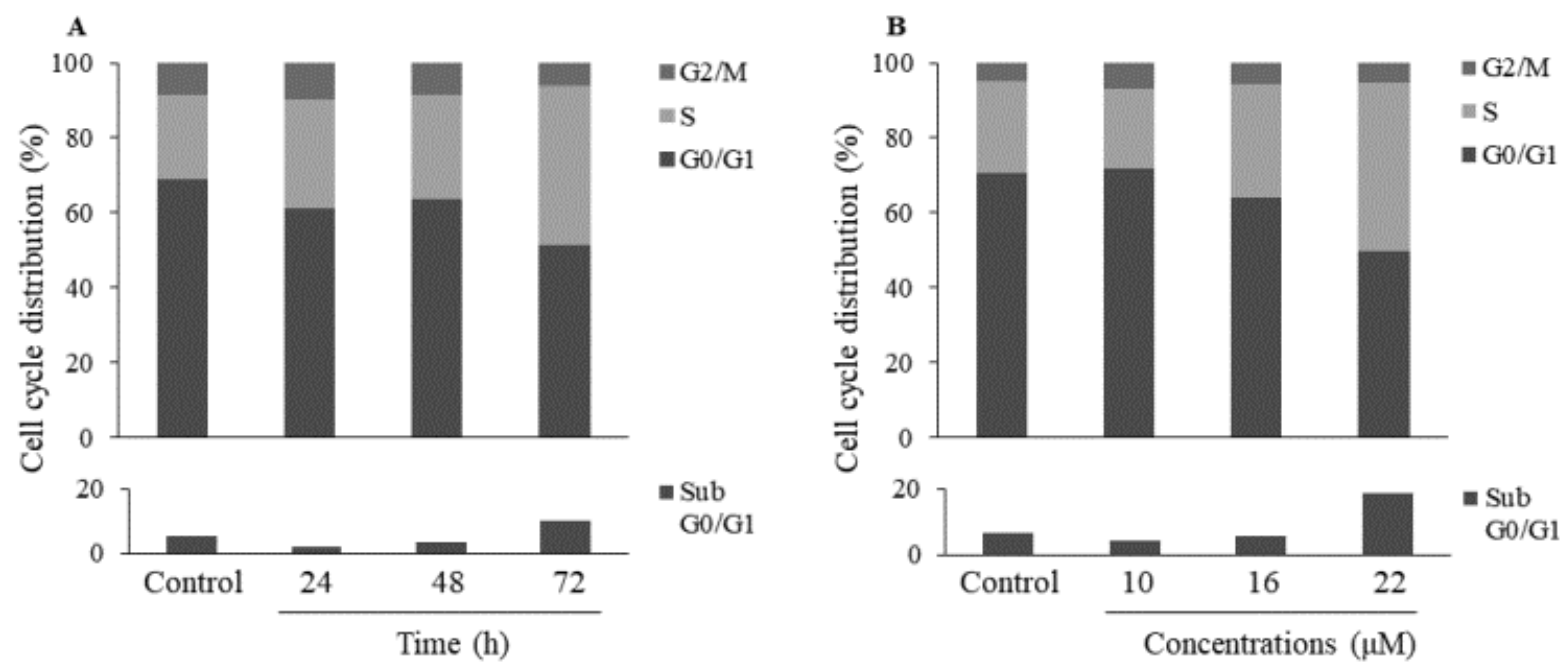

Figure S11. $\mathbf{2}_{\mathrm{mG}}$ compound induces increase of HEp2 cells in S phase of the cell cycle and an increase in SubG0/G1 cell population. Cells were treated either with $16 \mu \mathrm{M} \mathbf{2}_{\mathbf{m G}}$ during 24-72 $\mathrm{h}$ (A) or with different concentrations of $\mathbf{2}_{\mathbf{m G}}$ during $72 \mathrm{~h}$ (B). 

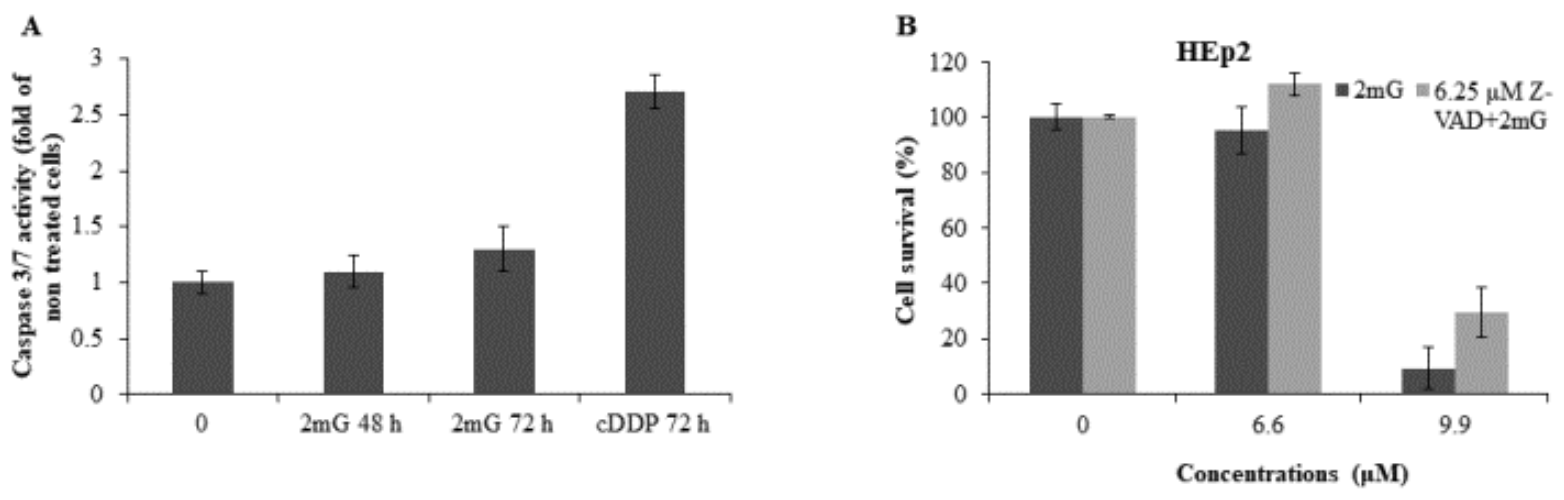

Figure S12. $2_{\mathrm{mG}}$ induces caspase independent cell death. HeLa cells were collected, lysed and preceded according to producer protocol for caspase $3 / 7$ activity assay after they were treated 48 and $72 \mathrm{~h}$ with $22 \mu \mathrm{M} \mathbf{2}_{\mathbf{m G}}$. Cells treated with $5 \mu \mathrm{M}$ cDDP for $72 \mathrm{~h}$ were used as positive control (A). HEp2 cells were pre-treated for two hours with $6.25 \mu \mathrm{M} Z$-VAD prior to treatment with different concentrations of $\mathbf{2}_{\mathbf{m G}}$. The cell survival was measured $72 \mathrm{~h}$ after the treatment by SRB assay (B). The experiments were repeated at least three times. 


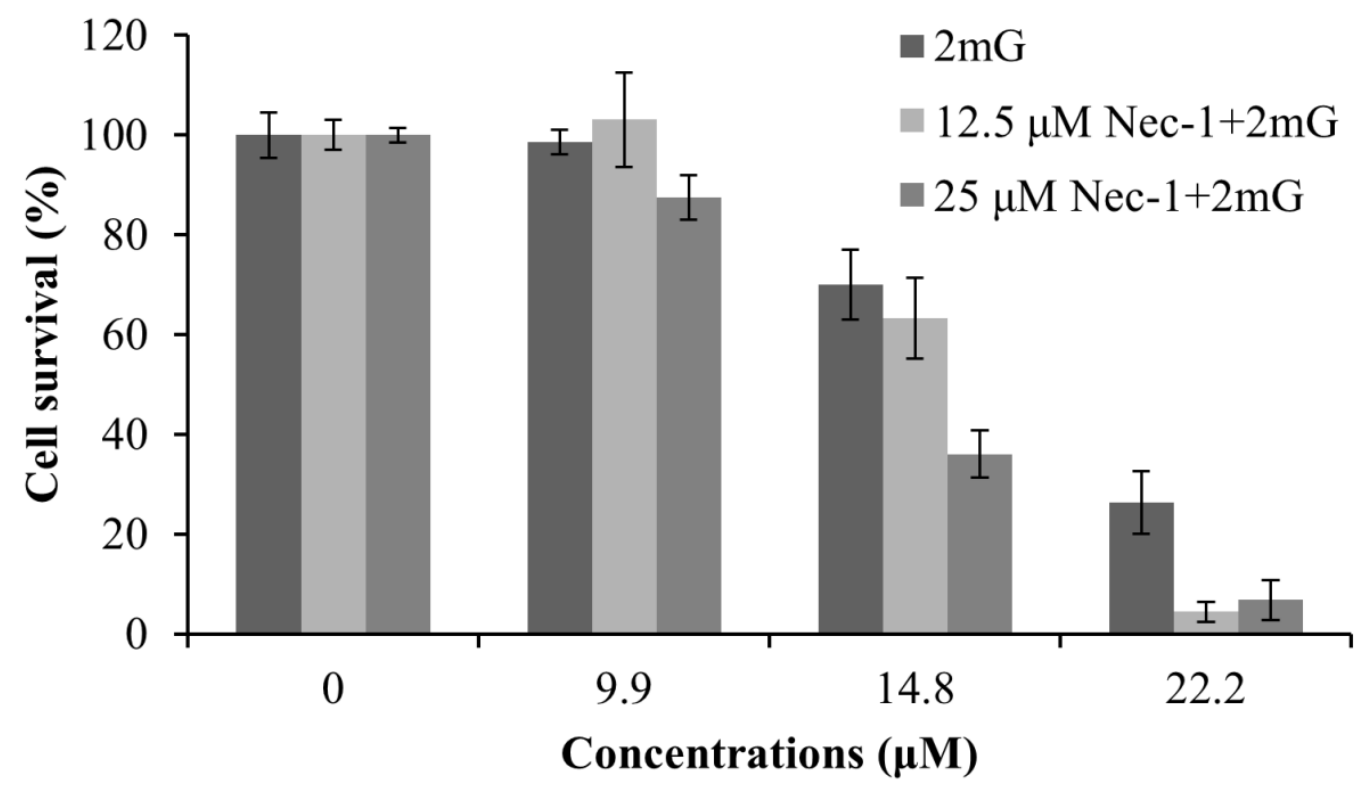

Figure S13. Necrostatin-1 (Nec-1) does not protect cells from $\mathbf{2}_{\mathrm{mG}}$ induced cell death. HeLa cells were pre-treated for two hours with either 12.5 or $25 \mu \mathrm{M} \mathrm{Nec}-1$ prior to treatment with different concentrations of $\mathbf{2}_{\mathbf{m G}}$. The cell survival was measured $72 \mathrm{~h}$ after the treatment by MTT assay. The experiment was repeated at least three times. 

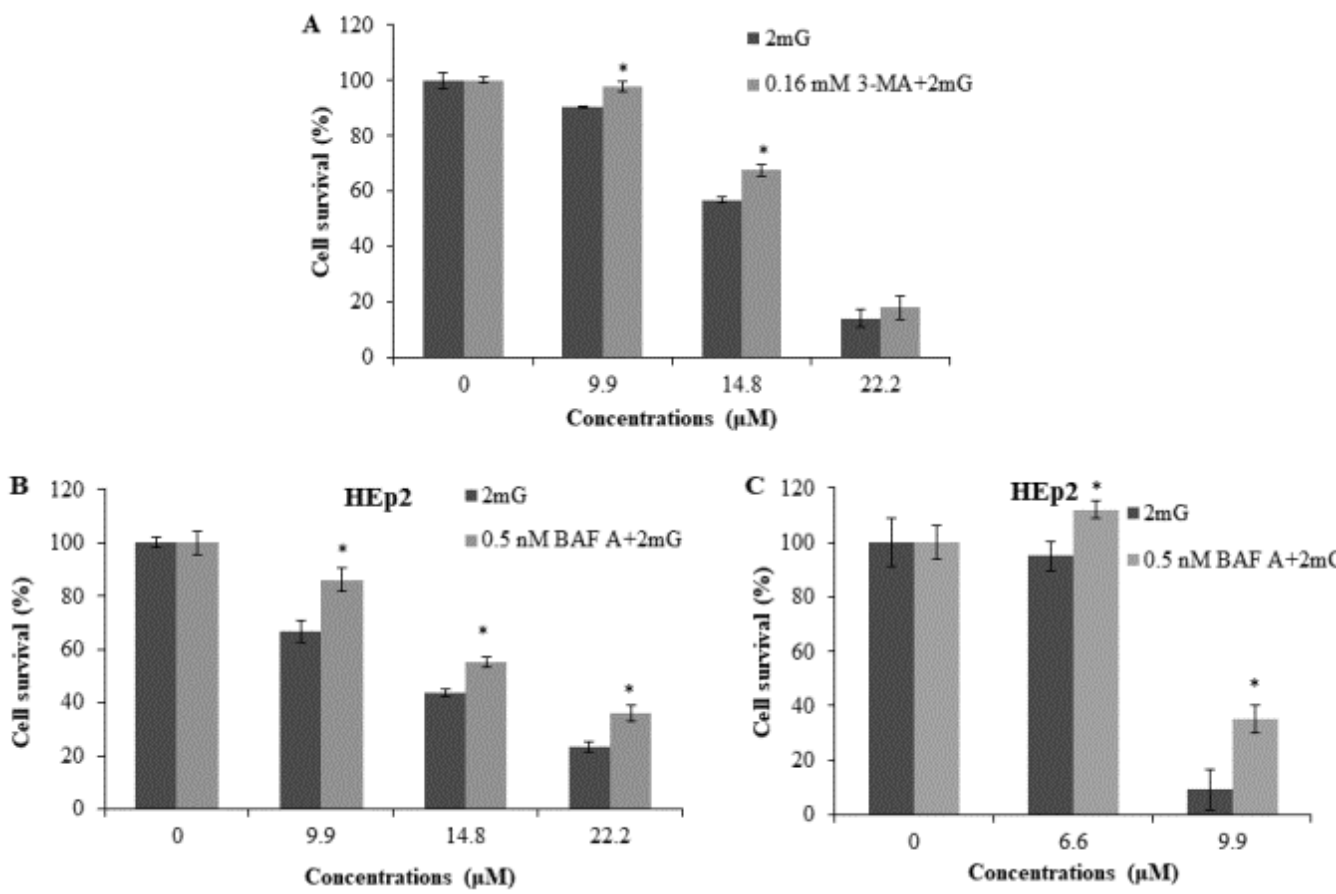

Figure S14. $2_{\mathrm{mG}}$ induces autophagy. HeLa cells were pre-treated for two hours with 0.16 $\mathrm{mM} 3$-MA prior to treatment with different concentrations of $\mathbf{2}_{\mathbf{m G}}$. The cell survival was measured $72 \mathrm{~h}$ after the treatment by MTT assay (A). HEp2 cells were pre-treated for two hours with $0.5 \mathrm{nM}$ BAF A prior to treatment with different concentrations of $\mathbf{2}_{\mathbf{m G}}$. The cell survival was measured $72 \mathrm{~h}$ after the treatment by MTT (B) and SRB assays (C). The experiments were repeated at least three times. Significance was determined between the $\mathbf{2}_{\mathbf{m G}}$ only and either 3 -MA or BAF A pre-treated and $\mathbf{2}_{\mathbf{m G}}$ treated cells $(*, \mathrm{P}<0.05)$. 
A

$60 \mathrm{~min}$

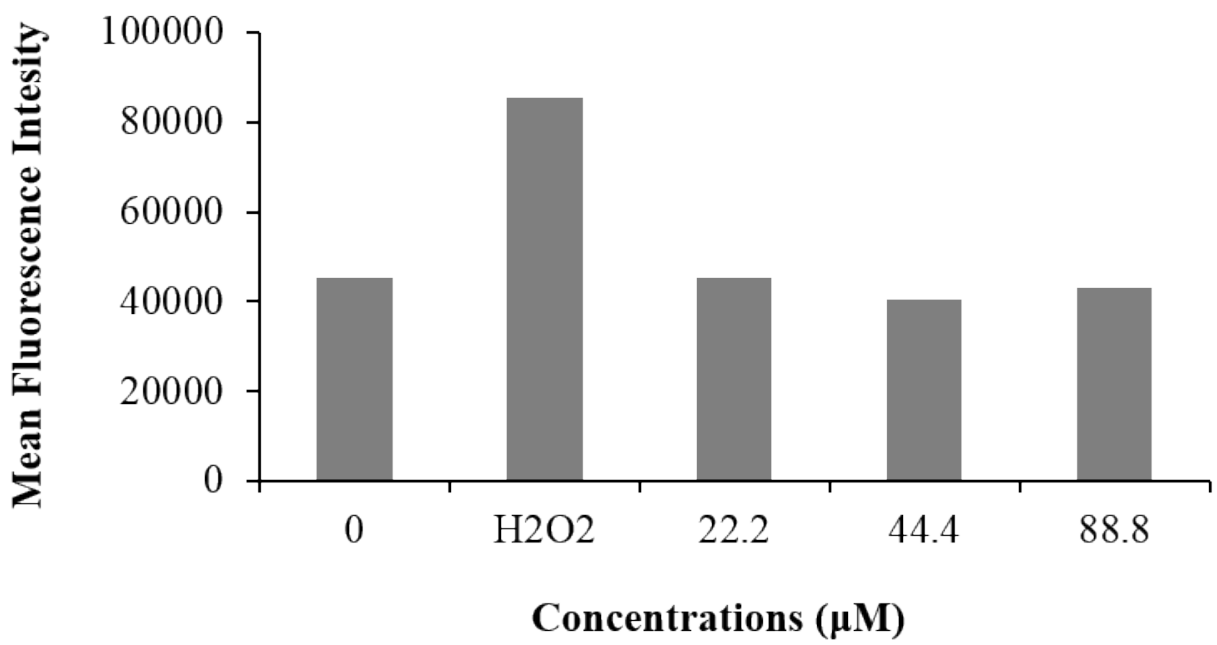

B

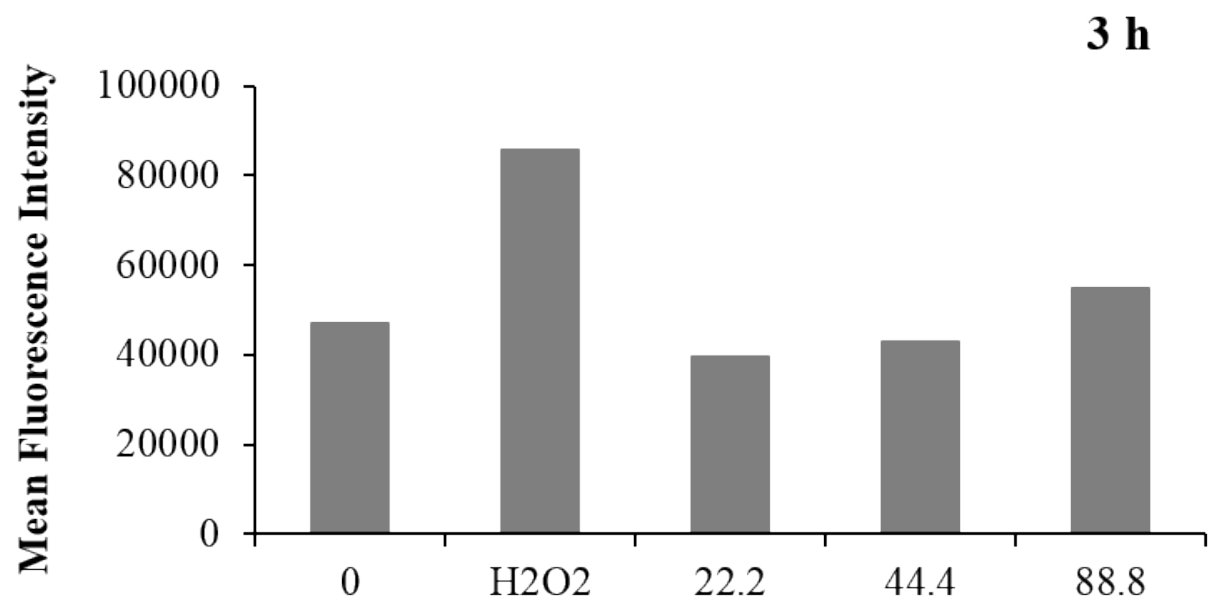

Concntrations $(\mu \mathrm{M})$

Figure S15. $2_{\mathrm{mG}}$ does not induce formation of ROS in HeLa cells. HeLa cells were stained for 1 hour with $10 \mathrm{mM} \mathrm{CM}-\mathrm{H}_{2}$ DCFDA and then treated with different concentrations of $\mathbf{2}_{\mathbf{m G}}$ during indicated time points (A, B). Afterward ROS formation was determined by flow cytometry. $\mathrm{H}_{2} \mathrm{O}_{2}$ was used as positive control. 
$\mathbf{A}$

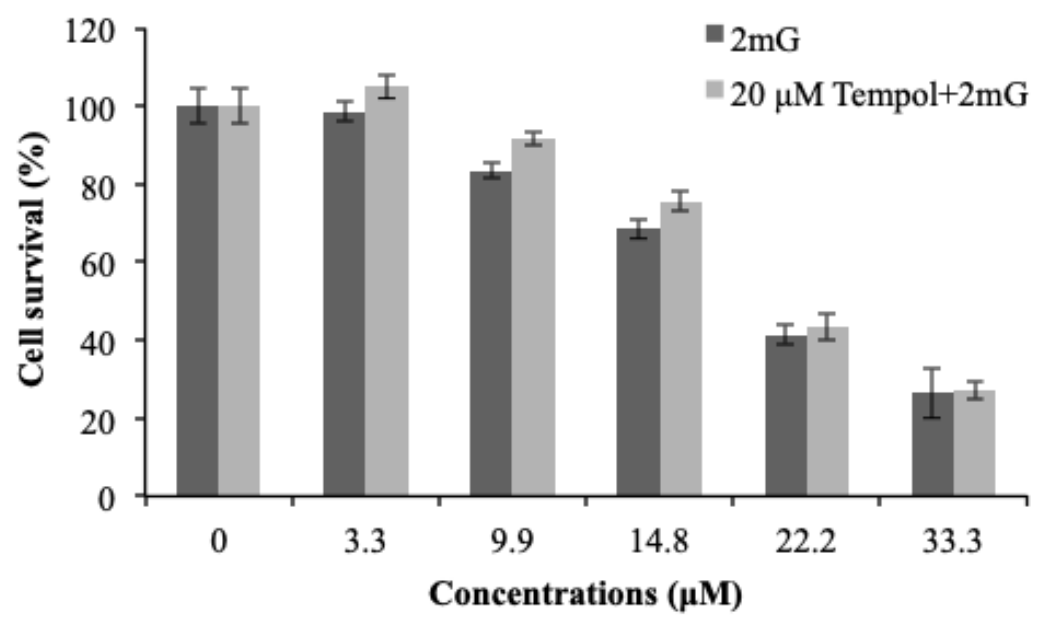

B

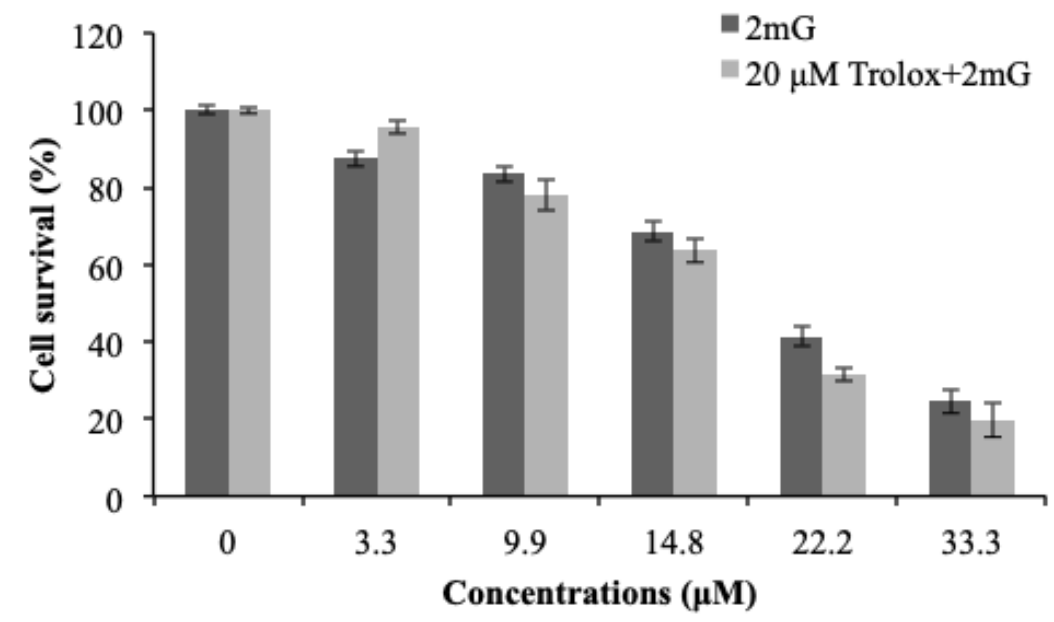

Figure S16. The antioxidants, tempol and trolox, do not influence cell survival upon $\mathbf{2}_{\mathrm{mG}}$ treatment. HeLa cells were pre-treated for two hours either with $20 \mu \mathrm{M}$ tempol (A) or $20 \mu \mathrm{M}$ trolox (B) prior to treatment with different concentrations of $\mathbf{2}_{\mathbf{m G}}$. The cell survival was measured $72 \mathrm{~h}$ after the treatment by MTT assay. The experiments were repeated at least three times. 
114

2mGs-I

\begin{tabular}{|c|c|c|c|}
\hline $\mathrm{C}$ & -1.323091 & 1.589268 & -2.574941 \\
\hline $\mathrm{C}$ & -1.808486 & 0.372239 & -3.079565 \\
\hline $\mathrm{C}$ & -3.199669 & 0.091252 & -2.908697 \\
\hline $\mathrm{C}$ & -4.052544 & 1.031244 & -2.327601 \\
\hline $\mathrm{C}$ & -3.577388 & 2.312134 & -1.891409 \\
\hline $\mathrm{C}$ & -2.201588 & 2.555061 & -1.998310 \\
\hline $\mathrm{Ru}$ & -2.430442 & 0.626465 & -0.812822 \\
\hline $\mathrm{Cl}$ & -4.349655 & 0.444883 & 0.644771 \\
\hline $\mathrm{C}$ & -0.931472 & -0.537325 & -3.887874 \\
\hline $\mathrm{C}$ & -4.541653 & 3.323075 & -1.349020 \\
\hline $\mathrm{P}$ & -0.989310 & 1.571440 & 0.892409 \\
\hline $\mathrm{C}$ & -1.817172 & 2.546003 & 2.205856 \\
\hline $\mathrm{C}$ & -3.058691 & 3.136319 & 1.966232 \\
\hline $\mathrm{C}$ & -3.621020 & 4.003647 & 2.899213 \\
\hline $\mathrm{C}$ & -2.947514 & 4.287640 & 4.082281 \\
\hline $\mathrm{C}$ & -1.704107 & 3.708123 & 4.327651 \\
\hline $\mathrm{C}$ & -1.138024 & 2.847755 & 3.394534 \\
\hline $\mathrm{C}$ & 0.113195 & 0.474672 & 1.881850 \\
\hline $\mathrm{C}$ & -0.481726 & -0.215381 & 2.941490 \\
\hline $\mathrm{C}$ & 0.237573 & -1.137845 & 3.689490 \\
\hline $\mathrm{C}$ & 1.572770 & -1.368124 & 3.397217 \\
\hline C & 2.201444 & -0.653286 & 2.376306 \\
\hline $\mathrm{C}$ & 1.472046 & 0.269222 & 1.622926 \\
\hline $\mathrm{C}$ & 3.662313 & -0.954717 & 2.181475 \\
\hline 0 & 4.188374 & -1.901745 & 2.755817 \\
\hline $\mathrm{N}$ & 4.403249 & -0.138911 & 1.388713 \\
\hline $\mathrm{C}$ & 5.833706 & -0.297346 & 1.413680 \\
\hline $\mathrm{H}$ & 6.271663 & 0.514161 & 0.826086 \\
\hline $\mathrm{C}$ & 6.291130 & -1.612255 & 0.805949 \\
\hline 0 & 5.809625 & -2.116298 & -0.194531 \\
\hline 0 & 7.326097 & -2.116510 & 1.449137 \\
\hline $\mathrm{C}$ & 7.856136 & -3.346046 & 0.936748 \\
\hline $\mathrm{C}$ & 0.129303 & 2.847166 & 0.164768 \\
\hline $\mathrm{C}$ & 1.076745 & 2.485556 & -0.797903 \\
\hline $\mathrm{C}$ & 1.828916 & 3.449935 & -1.459069 \\
\hline C & 1.655660 & 4.797291 & -1.158647 \\
\hline $\mathrm{C}$ & 0.734590 & 5.169677 & -0.184061 \\
\hline $\mathrm{C}$ & -0.025798 & 4.204966 & 0.473084 \\
\hline $\mathrm{P}$ & -1.948808 & -1.648739 & -0.210347 \\
\hline $\mathrm{C}$ & -2.634760 & -2.757350 & -1.503890 \\
\hline C & -4.030227 & -2.809253 & -1.628091 \\
\hline C & -4.615526 & -3.630184 & -2.583464 \\
\hline $\mathrm{C}$ & -3.816352 & -4.410025 & -3.421518 \\
\hline $\mathrm{C}$ & -2.433136 & -4.371820 & -3.292023 \\
\hline $\mathrm{C}$ & -1.840726 & -3.550552 & -2.331347 \\
\hline C & -0.154819 & -2.049914 & -0.219738 \\
\hline $\mathrm{C}$ & 0.657873 & -1.283151 & -1.047810 \\
\hline $\mathrm{C}$ & 2.021231 & -1.528779 & -1.174813 \\
\hline C & 2.590335 & -2.587424 & -0.463876 \\
\hline $\mathrm{C}$ & 1.792852 & -3.348116 & 0.383763 \\
\hline $\mathrm{C}$ & 0.432666 & -3.081598 & 0.518301 \\
\hline C & 2.790818 & -0.551480 & -2.012437 \\
\hline 0 & 2.207372 & 0.274788 & -2.723303 \\
\hline $\mathrm{N}$ & 4.135605 & -0.579687 & -1.918935 \\
\hline $\mathrm{C}$ & & 0 & -2 \\
\hline
\end{tabular}




\begin{tabular}{|c|c|c|c|}
\hline $\mathrm{H}$ & 4.513598 & 0.637378 & -3.571405 \\
\hline C & 4.915630 & 1.745374 & -1.788560 \\
\hline 0 & 4.571053 & 1.874740 & -0.631782 \\
\hline 0 & 5.382846 & 2.741022 & -2.530979 \\
\hline $\mathrm{C}$ & 5.536201 & 4.004697 & -1.869910 \\
\hline $\mathrm{C}$ & -2.606000 & -2.518452 & 1.276043 \\
\hline$C$ & -2.594167 & -3.922728 & 1.261916 \\
\hline $\mathrm{C}$ & -3.010905 & -4.649064 & 2.370977 \\
\hline $\mathrm{C}$ & -3.467146 & -3.986725 & 3.507667 \\
\hline $\mathrm{C}$ & -3.517285 & -2.597703 & 3.518580 \\
\hline $\mathrm{C}$ & -3.093651 & -1.869313 & 2.410294 \\
\hline $\mathrm{H}$ & 6.221423 & -0.240601 & 2.433756 \\
\hline $\mathrm{H}$ & 5.940793 & 0.101275 & -2.677327 \\
\hline $\mathrm{H}$ & 4.612761 & -1.163311 & -1.230928 \\
\hline $\mathrm{H}$ & 4.037841 & 0.635693 & 0.851576 \\
\hline $\mathrm{H}$ & 2.161431 & -2.087132 & 3.955343 \\
\hline $\mathrm{H}$ & -0.250360 & -1.670803 & 4.498532 \\
\hline $\mathrm{H}$ & -1.511666 & -0.014550 & 3.203161 \\
\hline $\mathrm{H}$ & 1.956555 & 0.824374 & 0.828417 \\
\hline $\mathrm{H}$ & 0.240247 & -0.443966 & -1.584302 \\
\hline $\mathrm{H}$ & -0.157270 & -3.671754 & 1.209174 \\
\hline $\mathrm{H}$ & 2.243297 & -4.142384 & 0.968573 \\
\hline $\mathrm{H}$ & 3.649239 & -2.811693 & -0.531503 \\
\hline $\mathrm{H}$ & -2.266901 & -4.460012 & 0.377568 \\
\hline $\mathrm{H}$ & -2.988434 & -5.733391 & 2.339689 \\
\hline $\mathrm{H}$ & -3.800471 & -4.552629 & 4.371460 \\
\hline $\mathrm{H}$ & -3.904451 & -2.071531 & 4.385015 \\
\hline $\mathrm{H}$ & -3.195235 & -0.794683 & 2.413536 \\
\hline $\mathrm{H}$ & -4.652793 & -2.203392 & -0.973310 \\
\hline $\mathrm{H}$ & -5.696354 & -3.669329 & -2.672203 \\
\hline $\mathrm{H}$ & -4.275163 & -5.052766 & -4.165506 \\
\hline $\mathrm{H}$ & -1.807827 & -4.986085 & -3.931594 \\
\hline $\mathrm{H}$ & -0.759836 & -3.537559 & -2.229292 \\
\hline $\mathrm{H}$ & 1.218712 & 1.451604 & -1.086099 \\
\hline $\mathrm{H}$ & 2.529147 & 3.132594 & -2.224230 \\
\hline $\mathrm{H}$ & 2.228834 & 5.554709 & -1.684302 \\
\hline $\mathrm{H}$ & -3.596519 & 2.901536 & 1.058901 \\
\hline $\mathrm{H}$ & -4.591195 & 4.447822 & 2.702749 \\
\hline $\mathrm{H}$ & -3.387063 & 4.959538 & 4.812220 \\
\hline $\mathrm{H}$ & -1.170804 & 3.929447 & 5.246219 \\
\hline $\mathrm{H}$ & -0.161013 & 2.419742 & 3.591449 \\
\hline $\mathrm{H}$ & 0.594618 & 6.217040 & 0.062486 \\
\hline $\mathrm{H}$ & -0.747962 & 4.521288 & 1.216626 \\
\hline $\mathrm{H}$ & 6.306292 & 3.934465 & -1.098881 \\
\hline $\mathrm{H}$ & 4.595776 & 4.310171 & -1.407864 \\
\hline $\mathrm{H}$ & 5.837239 & 4.706298 & -2.645032 \\
\hline $\mathrm{H}$ & 8.692935 & -3.595552 & 1.585518 \\
\hline $\mathrm{H}$ & 8.190528 & -3.215743 & -0.094226 \\
\hline $\mathrm{H}$ & 7.093323 & -4.125255 & 0.976030 \\
\hline $\mathrm{H}$ & -3.593726 & -0.866243 & -3.226860 \\
\hline $\mathrm{H}$ & -5.093507 & 0.777496 & -2.161682 \\
\hline $\mathrm{H}$ & -1.788598 & 3.486414 & -1.62625 \\
\hline $\mathrm{H}$ & -0.269014 & 1.811726 & -2.673930 \\
\hline $\mathrm{H}$ & -5.166844 & 3.701279 & -2.163890 \\
\hline $\mathrm{H}$ & -4.021689 & 4.169575 & -0.896189 \\
\hline $\mathrm{H}$ & -5.197400 & 2.861136 & -0.604949 \\
\hline $\mathrm{H}$ & -1.155212 & -0.356834 & -4.94520 \\
\hline
\end{tabular}




\begin{tabular}{|c|c|c|c|}
\hline $\mathrm{H}$ & 0.128711 & -0.332634 & -3.724170 \\
\hline $\mathrm{H}$ & -1.142574 & -1.587771 & -3.683865 \\
\hline & & & \\
\hline \multicolumn{4}{|c|}{$2 m G s-I b$} \\
\hline $\mathrm{C}$ & -1.298360 & -1.024214 & 2.875145 \\
\hline $\mathrm{C}$ & -1.660456 & 0.303289 & 3.162132 \\
\hline $\mathrm{C}$ & -3.044986 & 0.648039 & 3.081156 \\
\hline $\mathrm{C}$ & -4.004959 & -0.321731 & 2.779394 \\
\hline $\mathrm{C}$ & -3.649995 & -1.689681 & 2.541272 \\
\hline $\mathrm{C}$ & -2.285500 & -2.009957 & 2.579604 \\
\hline Ru & -2.534520 & -0.321290 & 1.069266 \\
\hline $\mathrm{Cl}$ & -4.618446 & -0.102123 & -0.154073 \\
\hline $\mathrm{C}$ & -0.648303 & 1.291077 & 3.662147 \\
\hline $\mathrm{C}$ & -4.709701 & -2.713400 & 2.264374 \\
\hline $\mathrm{P}$ & -1.388584 & -1.691739 & -0.575760 \\
\hline $\mathrm{C}$ & -2.453215 & -2.635245 & -1.731396 \\
\hline $\mathrm{C}$ & -3.750543 & -2.984217 & -1.349108 \\
\hline $\mathrm{C}$ & -4.522573 & -3.820949 & -2.150390 \\
\hline $\mathrm{C}$ & -4.004684 & -4.321018 & -3.340226 \\
\hline $\mathrm{C}$ & -2.705060 & -3.993269 & -3.721362 \\
\hline $\mathrm{C}$ & -1.930079 & -3.162424 & -2.920751 \\
\hline $\mathrm{C}$ & -0.125329 & -0.959702 & -1.715648 \\
\hline $\mathrm{C}$ & -0.539556 & -0.335981 & -2.898745 \\
\hline $\mathrm{C}$ & 0.376426 & 0.265361 & -3.751758 \\
\hline $\mathrm{C}$ & 1.731953 & 0.255111 & -3.443082 \\
\hline $\mathrm{C}$ & 2.166416 & -0.377416 & -2.279681 \\
\hline $\mathrm{C}$ & 1.239432 & -0.992023 & -1.438577 \\
\hline $\mathrm{C}$ & 3.610677 & -0.489641 & -1.876931 \\
\hline 0 & 3.988002 & -1.354473 & -1.097185 \\
\hline $\mathrm{N}$ & 4.463920 & 0.412006 & -2.441274 \\
\hline $\mathrm{C}$ & 5.885053 & 0.352576 & -2.229053 \\
\hline $\mathrm{H}$ & 6.428557 & 0.338917 & -3.178395 \\
\hline $\mathrm{C}$ & 6.411321 & 1.484863 & -1.369475 \\
\hline 0 & 5.726063 & 2.227187 & -0.697060 \\
\hline 0 & 7.729822 & 1.551928 & -1.449320 \\
\hline $\mathrm{C}$ & 8.364116 & 2.466363 & -0.547566 \\
\hline $\mathrm{C}$ & -0.460934 & -3.056884 & 0.253752 \\
\hline $\mathrm{C}$ & 0.609716 & -2.771574 & 1.111597 \\
\hline $\mathrm{C}$ & 1.278678 & -3.789350 & 1.780780 \\
\hline $\mathrm{C}$ & 0.884302 & -5.114090 & 1.614215 \\
\hline $\mathrm{C}$ & -0.179436 & -5.409854 & 0.768442 \\
\hline $\mathrm{C}$ & -0.846721 & -4.392185 & 0.088705 \\
\hline $\mathrm{P}$ & -1.933152 & 1.728586 & 0.005711 \\
\hline $\mathrm{C}$ & -2.476690 & 3.135304 & 1.052653 \\
\hline $\mathrm{C}$ & -3.855459 & 3.295967 & 1.251151 \\
\hline $\mathrm{C}$ & -4.328978 & 4.337088 & 2.039403 \\
\hline $\mathrm{C}$ & -3.435231 & 5.228732 & 2.635065 \\
\hline $\mathrm{C}$ & -2.068604 & 5.080098 & 2.430496 \\
\hline $\mathrm{C}$ & -1.587710 & 4.038579 & 1.635764 \\
\hline $\mathrm{C}$ & -0.123165 & 1.990661 & -0.148146 \\
\hline $\mathrm{C}$ & 0.698168 & 1.242106 & 0.684103 \\
\hline $\mathrm{C}$ & 2.078536 & 1.412100 & 0.710130 \\
\hline $\mathrm{C}$ & 2.658676 & 2.374847 & -0.114258 \\
\hline $\mathrm{C}$ & 1.848202 & 3.118635 & -0.969110 \\
\hline $\mathrm{C}$ & 0.470432 & 2.926429 & -1.002155 \\
\hline $\mathrm{C}$ & 2.823960 & 0.439474 & 1.577044 \\
\hline 0 & 2.206550 & -0.289790 & 2.36445 \\
\hline
\end{tabular}




\begin{tabular}{|c|c|c|c|}
\hline $\mathrm{N}$ & 4.157809 & 0.336849 & 1.437912 \\
\hline $\mathrm{C}$ & 4.818215 & -0.779990 & 2.068120 \\
\hline $\mathrm{H}$ & 4.295064 & -1.713389 & 1.832042 \\
\hline$C$ & 6.243215 & -0.905649 & 1.590653 \\
\hline O & 6.778878 & -0.175975 & 0.786512 \\
\hline 0 & 6.839103 & -1.936009 & 2.179622 \\
\hline 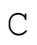 & 8.201555 & -2.169015 & 1.803972 \\
\hline & -2.636347 & 2.252314 & -1.610256 \\
\hline$C$ & -2.621664 & 3.617124 & -1.933079 \\
\hline$C$ & -3.094639 & 4.061485 & -3.162850 \\
\hline$C$ & -3.607735 & 3.151072 & -4.082384 \\
\hline C & -3.658595 & 1.799583 & -3.757766 \\
\hline C & -3.182019 & 1.354172 & -2.528553 \\
\hline $\mathrm{H}$ & 6.104561 & -0.577792 & -1.698698 \\
\hline 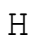 & 4.815472 & -0.681182 & 3.158464 \\
\hline$H$ & 4.669558 & 0.838662 & 0.718844 \\
\hline $\mathrm{H}$ & 4.082573 & 1.219900 & -2.905626 \\
\hline $\mathrm{H}$ & 2.438183 & 0.697389 & -4.139051 \\
\hline H & 0.026680 & 0.728988 & -4.667818 \\
\hline $\mathrm{H}$ & -1.580672 & -0.343321 & -3.186050 \\
\hline$H$ & 1.641539 & -1.501982 & -0.575220 \\
\hline $\mathrm{H}$ & 0.276915 & 0.468861 & 1.309223 \\
\hline $\mathrm{H}$ & -0.132090 & 3.503696 & -1.693774 \\
\hline $\mathrm{H}$ & 2.299542 & 3.856628 & -1.624527 \\
\hline $\mathrm{H}$ & 3.730114 & 2.537915 & -0.131034 \\
\hline $\mathrm{H}$ & -2.260235 & 4.346596 & -1.214935 \\
\hline $\mathrm{H}$ & -3.074338 & 5.121563 & -3.393148 \\
\hline $\mathrm{H}$ & -3.988207 & 3.498103 & -5.037604 \\
\hline $\mathrm{H}$ & -4.089036 & 1.085599 & -4.452457 \\
\hline $\mathrm{H}$ & -3.288527 & 0.309636 & -2.268507 \\
\hline $\mathrm{H}$ & -4.551259 & 2.600311 & 0.787738 \\
\hline $\mathrm{H}$ & -5.397245 & 4.458808 & 2.185759 \\
\hline $\mathrm{H}$ & -3.807576 & 6.041031 & 3.250527 \\
\hline $\mathrm{H}$ & -1.369769 & 5.777243 & 2.881107 \\
\hline $\mathrm{H}$ & -0.519195 & 3.938152 & 1.472384 \\
\hline $\mathrm{H}$ & 0.940834 & -1.754337 & 1.288099 \\
\hline $\mathrm{H}$ & 2.104437 & -3.532593 & 2.436306 \\
\hline $\mathrm{H}$ & 1.402171 & -5.910479 & 2.138494 \\
\hline $\mathrm{H}$ & -4.167021 & -2.586699 & -0.433878 \\
\hline $\mathrm{H}$ & -5.533037 & -4.071739 & -1.845123 \\
\hline $\mathrm{H}$ & -4.608629 & -4.968020 & -3.968012 \\
\hline $\mathrm{H}$ & -2.288265 & -4.392924 & -4.639938 \\
\hline $\mathrm{H}$ & -0.911254 & -2.940421 & -3.218535 \\
\hline $\mathrm{H}$ & -0.493764 & -6.438698 & 0.626725 \\
\hline $\mathrm{H}$ & -1.669211 & -4.651979 & -0.567512 \\
\hline $\mathrm{H}$ & 8.821078 & -1.310490 & 2.071304 \\
\hline $\mathrm{H}$ & 8.275867 & -2.345866 & 0.729270 \\
\hline $\mathrm{H}$ & 8.511244 & -3.051569 & 2.359940 \\
\hline $\mathrm{H}$ & 9.430836 & 2.375923 & -0.742513 \\
\hline $\mathrm{H}$ & 8.133301 & 2.183019 & 0.480909 \\
\hline $\mathrm{H}$ & 8.023275 & 3.486302 & -0.734257 \\
\hline $\mathrm{H}$ & -3.350444 & 1.675664 & 3.238520 \\
\hline $\mathrm{H}$ & -5.040901 & -0.024141 & 2.664511 \\
\hline $\mathrm{H}$ & -1.966403 & -3.021917 & 2.356358 \\
\hline $\mathrm{H}$ & -0.252520 & -1.299240 & 2.920772 \\
\hline $\mathrm{H}$ & -5.224641 & -2.971944 & 3.194907 \\
\hline $\mathrm{H}$ & -4.277457 & -3.624965 & 1.846123 \\
\hline
\end{tabular}




\begin{tabular}{|c|c|c|c|}
\hline $\mathrm{H}$ & $-5 \quad 449097$ & $-2 \quad 315179$ & 1049 \\
\hline $\mathrm{H}$ & -0.788869 & 1.394308 & 4.743576 \\
\hline $\mathrm{H}$ & 0.371649 & 0.948284 & 3.478288 \\
\hline $\mathrm{H}$ & -0.795700 & 2.276631 & 3.218242 \\
\hline & & & \\
\hline $2 \mathrm{mc}$ & & & \\
\hline $\mathrm{C}$ & 0.752685 & -3.832364 & 0.773832 \\
\hline $\mathrm{C}$ & 1.104634 & -2.495432 & 1.021830 \\
\hline $\mathrm{C}$ & 0.651470 & -1.884203 & 2.184317 \\
\hline $\mathrm{C}$ & -0.139773 & -2.580577 & 3.097011 \\
\hline $\mathrm{C}$ & -0.476321 & -3.904106 & 2.850710 \\
\hline $\mathrm{C}$ & -0.025974 & -4.531116 & 1.685910 \\
\hline $\mathrm{P}$ & 2.023040 & -1.541996 & -0.246745 \\
\hline $\mathrm{Ru}$ & 2.810521 & 0.619300 & 0.358351 \\
\hline $\mathrm{Cl}$ & 3.242078 & 0.895525 & -2.001327 \\
\hline $\mathrm{C}$ & 0.906653 & -1.822730 & -1.676814 \\
\hline $\mathrm{C}$ & 1.328655 & -2.001648 & -2.994858 \\
\hline $\mathrm{C}$ & 0.396648 & -2.260974 & -4.000016 \\
\hline $\mathrm{C}$ & -0.957296 & -2.359990 & -3.704582 \\
\hline $\mathrm{C}$ & -1.383512 & -2.200159 & -2.387297 \\
\hline $\mathrm{C}$ & -0.458765 & -1.903120 & -1.392911 \\
\hline $\mathrm{H}$ & 0.740150 & -2.397772 & -5.019828 \\
\hline $\mathrm{C}$ & 3.530431 & -2.562579 & -0.531840 \\
\hline $\mathrm{C}$ & 3.921606 & -3.535388 & 0.395983 \\
\hline $\mathrm{C}$ & 5.143730 & -4.192349 & 0.262210 \\
\hline $\mathrm{C}$ & 5.989848 & -3.892195 & -0.799645 \\
\hline $\mathrm{C}$ & 5.611360 & -2.922415 & -1.725359 \\
\hline $\mathrm{C}$ & 4.399287 & -2.255404 & -1.588950 \\
\hline $\mathrm{P}$ & 0.773904 & 1.827776 & 0.033241 \\
\hline $\mathrm{C}$ & -0.163433 & 1.922440 & 1.606329 \\
\hline $\mathrm{C}$ & -0.987350 & 0.853684 & 1.976051 \\
\hline $\mathrm{C}$ & -1.662554 & 0.863349 & 3.191378 \\
\hline $\mathrm{C}$ & -1.515624 & 1.939017 & 4.063325 \\
\hline $\mathrm{C}$ & -0.700857 & 3.010375 & 3.705466 \\
\hline $\mathrm{C}$ & -0.031267 & 3.005026 & 2.484031 \\
\hline $\mathrm{C}$ & -0.494216 & 1.393645 & -1.221218 \\
\hline $\mathrm{C}$ & -0.094239 & 1.142064 & -2.540520 \\
\hline $\mathrm{C}$ & -1.044207 & 0.947992 & -3.534142 \\
\hline $\mathrm{C}$ & -2.401441 & 0.994104 & -3.232548 \\
\hline $\mathrm{C}$ & -2.805139 & 1.280997 & -1.931360 \\
\hline $\mathrm{C}$ & -1.854858 & 1.497327 & -0.936353 \\
\hline $\mathrm{H}$ & -0.720078 & 0.753307 & -4.550571 \\
\hline $\mathrm{C}$ & 1.049964 & 3.589525 & -0.420407 \\
\hline $\mathrm{C}$ & -0.022066 & 4.488420 & -0.319050 \\
\hline $\mathrm{C}$ & 0.131854 & 5.811933 & -0.710241 \\
\hline C & 1.349728 & 6.253029 & -1.226225 \\
\hline $\mathrm{C}$ & 2.405342 & 5.359520 & -1.362850 \\
\hline $\mathrm{C}$ & 2.255739 & 4.031996 & -0.966281 \\
\hline $\mathrm{C}$ & 3.074271 & 0.761445 & 2.656696 \\
\hline $\mathrm{C}$ & 3.795448 & -0.333992 & 2.126029 \\
\hline $\mathrm{C}$ & 4.807481 & -0.166594 & 1.140707 \\
\hline $\mathrm{C}$ & 5.070541 & 1.104751 & 0.600721 \\
\hline $\mathrm{C}$ & 4.280815 & 2.204542 & 1.046398 \\
\hline $\mathrm{C}$ & 3.302330 & 2.027506 & 2.044891 \\
\hline C & 2.204308 & 0.627639 & 3.874480 \\
\hline $\mathrm{C}$ & 6.127722 & 1.293826 & -0.44303 \\
\hline $\mathrm{H}$ & -3.142803 & 0.831942 & -4.00742 \\
\hline
\end{tabular}




\begin{tabular}{|c|c|c|c|}
\hline $\mathrm{H}$ & 0.958615 & 1.096729 & -2.787736 \\
\hline $\mathrm{H}$ & -2.221554 & 1.760651 & 0.047978 \\
\hline $\mathrm{C}$ & -4.244192 & 1.454181 & -1.552121 \\
\hline $\mathrm{H}$ & -1.688144 & -2.566696 & -4.479178 \\
\hline $\mathrm{H}$ & 2.377934 & -1.945715 & -3.253690 \\
\hline $\mathrm{H}$ & -0.822505 & -1.727497 & -0.385494 \\
\hline $\mathrm{C}$ & -2.831130 & -2.323366 & -2.021704 \\
\hline $\mathrm{H}$ & 3.282762 & -3.786400 & 1.235342 \\
\hline $\mathrm{H}$ & 5.429480 & -4.941616 & 0.993057 \\
\hline $\mathrm{H}$ & 6.939645 & -4.405987 & -0.904091 \\
\hline $\mathrm{H}$ & 6.267095 & -2.672686 & -2.553256 \\
\hline $\mathrm{H}$ & 4.158948 & -1.453321 & -2.276245 \\
\hline $\mathrm{H}$ & -0.983312 & 4.155774 & 0.061216 \\
\hline $\mathrm{H}$ & -0.703785 & 6.498180 & -0.621794 \\
\hline $\mathrm{H}$ & 1.466804 & 7.286557 & -1.535162 \\
\hline $\mathrm{H}$ & 3.345161 & 5.687672 & -1.795001 \\
\hline $\mathrm{H}$ & 3.061011 & 3.327859 & -1.133015 \\
\hline $\mathrm{H}$ & 3.598177 & -1.329373 & 2.512651 \\
\hline $\mathrm{H}$ & 5.345774 & -1.031489 & 0.771974 \\
\hline $\mathrm{H}$ & 4.429254 & 3.184402 & 0.610635 \\
\hline $\mathrm{H}$ & 2.713319 & 2.880410 & 2.365310 \\
\hline $\mathrm{H}$ & 7.051560 & 1.623379 & 0.044130 \\
\hline $\mathrm{H}$ & 5.827758 & 2.046254 & -1.173564 \\
\hline $\mathrm{H}$ & 6.323701 & 0.359394 & -0.971207 \\
\hline $\mathrm{H}$ & 2.684721 & 1.153371 & 4.705716 \\
\hline $\mathrm{H}$ & 1.217531 & 1.069456 & 3.729716 \\
\hline $\mathrm{H}$ & 2.089078 & -0.417481 & 4.165477 \\
\hline $\mathrm{H}$ & -1.147093 & 0.011795 & 1.311475 \\
\hline $\mathrm{H}$ & -2.316293 & 0.029518 & 3.423536 \\
\hline $\mathrm{H}$ & -2.044253 & 1.952458 & 5.010880 \\
\hline $\mathrm{H}$ & -0.592600 & 3.859615 & 4.372105 \\
\hline $\mathrm{H}$ & 0.575103 & 3.862546 & 2.209566 \\
\hline $\mathrm{H}$ & -0.503867 & -2.074470 & 3.985011 \\
\hline $\mathrm{H}$ & -1.093698 & -4.451595 & 3.555300 \\
\hline $\mathrm{H}$ & -0.283436 & -5.567119 & 1.490716 \\
\hline $\mathrm{H}$ & 1.083724 & -4.322138 & -0.137316 \\
\hline $\mathrm{H}$ & 0.876823 & -0.841780 & 2.357876 \\
\hline 0 & -3.732741 & -1.894253 & -2.735057 \\
\hline $\mathrm{N}$ & -3.044588 & -2.956813 & -0.840190 \\
\hline $\mathrm{H}$ & -2.236426 & -3.282170 & -0.331312 \\
\hline $\mathrm{C}$ & -4.272808 & -2.857298 & -0.100832 \\
\hline $\mathrm{C}$ & -4.027743 & -2.168710 & 1.227831 \\
\hline $\mathrm{H}$ & -4.973501 & -2.233117 & -0.661312 \\
\hline $\mathrm{H}$ & -4.742137 & -3.831384 & 0.064505 \\
\hline 0 & -5.056807 & -2.339223 & 2.045434 \\
\hline 0 & -3.020113 & -1.551445 & 1.503081 \\
\hline $\mathrm{C}$ & -5.054908 & -1.533370 & 3.222996 \\
\hline $\mathrm{H}$ & -5.994141 & -1.748270 & 3.729527 \\
\hline $\mathrm{H}$ & -4.210786 & -1.793386 & 3.867211 \\
\hline $\mathrm{H}$ & -5.003454 & -0.478312 & 2.945332 \\
\hline 0 & -4.596049 & 2.307716 & -0.748915 \\
\hline $\mathrm{N}$ & -5.126291 & 0.622083 & -2.172041 \\
\hline $\mathrm{H}$ & -4.776576 & -0.257779 & -2.541114 \\
\hline $\mathrm{C}$ & -6.511626 & 0.731768 & -1.806385 \\
\hline $\mathrm{H}$ & -7.102576 & 0.043999 & -2.417573 \\
\hline $\mathrm{C}$ & -6.754405 & 0.402103 & -0.340308 \\
\hline $\mathrm{H}$ & -6.878840 & 1.743512 & -1.99154 \\
\hline
\end{tabular}




$$
\begin{array}{ll}
\mathrm{O} & -7.902671 \\
\mathrm{O} & -6.028829 \\
\mathrm{C} & -8.252497 \\
\mathrm{H} & -9.219739 \\
\mathrm{H} & -8.320685 \\
\mathrm{H} & -7.506514 \\
\multicolumn{1}{l}{114} &
\end{array}
$$$$
2 \mathrm{mGa}-\mathrm{I}
$$$$
\text { C } \quad-7.185370
$$$$
\text { o } \quad-7.018305
$$$$
\text { C } \quad-6.658262
$$$$
\text { o }-6.488289
$$$$
\text { C } \quad-5.983837
$$$$
\text { C } \quad-5.905962
$$$$
\text { C } \quad-6.348414
$$$$
\text { C } \quad-6.274107
$$$$
\text { O } \quad-5.904048
$$$$
\text { O } \quad-5.680822
$$$$
\mathrm{N} \quad-5.101380
$$$$
\text { C } \quad-3.894765
$$$$
\mathrm{N} \quad-4.858600
$$$$
\text { C } \quad-3.574234
$$$$
\text { o } \quad-3.799268
$$$$
\text { O } \quad-3.309999
$$$$
\mathrm{H} \quad-6.864188
$$$$
\mathrm{H} \quad-5.776496
$$$$
\mathrm{H} \quad-6.318303
$$$$
\mathrm{H} \quad-7.158405
$$$$
\mathrm{H} \quad-5.198506
$$$$
\mathrm{H} \quad-5.112328
$$$$
\text { C } \quad-2.483244
$$$$
\text { C } \quad-2.691202
$$$$
\mathrm{H} \quad-3.689414
$$$$
\text { C } \quad-1.595311
$$$$
\mathrm{H} \quad-1.750633
$$$$
\text { C } \quad-0.303100
$$$$
\mathrm{H} \quad 0.540102
$$$$
\text { C } \quad-0.085522
$$$$
\begin{array}{ll}
\mathrm{P} & -0.085522 \\
\mathrm{C} & 1.648636
\end{array}
$$$$
\text { C } \quad-1.182739
$$$$
\mathrm{H} \quad-1.083813
$$$$
\text { C } \quad-2.674981
$$$$
\text { C } \quad-1.449735
$$$$
\mathrm{H} \quad-1.478488
$$$$
\text { C } \quad-0.251257
$$$$
\text { 1.377211 }
$$$$
-0.296188
$$$$
0.630454
$$$$
-1.511373
$$$$
-1.531886
$$$$
-2.707637
$$$$
-3.644923
$$$$
0.134359
$$$$
0.254523
$$$$
-0.242007
$$$$
-0.730900
$$$$
-0.116525
$$

$$
\begin{array}{r}
0.928000 \\
-0.277952 \\
0.653971 \\
1.127815 \\
-0.423185 \\
1.078095
\end{array}
$$

2.034360

2.318606

$-2.781913$

$-3.045756$

$-2.062048$

$-2.442630$

1.423445

1.815466

0.392816

$-0.976816$

1.305986

1.780765

$-1.758451$

$-2.064650$

2.600096

$-2.858405$

$-2.186727$

$-3.525590$

2.904989

1.408633

0.494641

$-0.992300$

$-1.418909$

$-0.559351$

$-0.278867$

$-0.042650$

0.623829

$-0.372948$

$-0.001699$

$-1.209362$

$-1.589957$

$-1.738832$

$-2.427815$

1.277027

1.667065

2.283727

1.262957

1.789016

0.442321

0.108282

0.054209

$-0.584364$

0.472876

0.149846

4.367370

4.122839

5.004719

5.917518

4.713648
0.079270

0.349213

1.436741

1.593767

1.605629

2.112720

3.610995

2.216442

$-3.923447$

$-2.525757$

$-1.800553$

$-0.329839$

1.512164

0.043612

1.984419

$-2.261726$

$-0.610084$

$-0.213537$

0.374926

0.067455

0.694149

$-0.831074$

0.132283

$-0.268656$

$-0.019130$

$-0.456051$

$-1.216398$

0.994019

0.878476

1.957156

2.277059

2.644469

3.485782

2.258750

2.829243

1.158201

0.705373

0.488809

$-0.338863$

$-0.937569$

$-0.395267$

0.493840

$-0.973730$

$-0.309318$

$-2.104954$

$-2.554670$

$-2.653561$

$-3.530770$

$-2.075617$

$-2.515108$

$-0.373797$

0.676111

1.625962

1.303240

2. 981945 


\begin{tabular}{|c|c|c|c|}
\hline $\mathrm{H}$ & -0.498903 & 5.405352 & 3.725393 \\
\hline C & 0.507475 & 3.536574 & 3.374322 \\
\hline $\mathrm{H}$ & 0.627460 & 3.306085 & 4.427871 \\
\hline $\mathrm{C}$ & 1.000638 & 2.646777 & 2.422619 \\
\hline $\mathrm{H}$ & 1.517397 & 1.759180 & 2.749414 \\
\hline $\mathrm{C}$ & 0.881087 & 2.925061 & 1.061098 \\
\hline C & 1.961671 & 3.057742 & -1.507650 \\
\hline $\mathrm{C}$ & 3.030975 & 3.867707 & -1.098024 \\
\hline $\mathrm{H}$ & 3.444782 & 3.750137 & -0.099224 \\
\hline $\mathrm{C}$ & 3.563047 & 4.815866 & -1.961819 \\
\hline $\mathrm{H}$ & 4.386700 & 5.441931 & -1.634286 \\
\hline $\mathrm{C}$ & 3.033027 & 4.970069 & -3.244603 \\
\hline $\mathrm{H}$ & 3.447650 & 5.711782 & -3.919165 \\
\hline $\mathrm{C}$ & 1.960262 & 4.183885 & -3.647390 \\
\hline $\mathrm{H}$ & 1.527764 & 4.315368 & -4.633909 \\
\hline $\mathrm{C}$ & 1.419657 & 3.233055 & -2.779199 \\
\hline $\mathrm{H}$ & 0.565077 & 2.644953 & -3.097909 \\
\hline C & 1.531765 & -3.022087 & -0.444658 \\
\hline $\mathrm{C}$ & 0.867737 & -2.886750 & -1.672126 \\
\hline $\mathrm{H}$ & 0.363035 & -1.958974 & -1.914376 \\
\hline $\mathrm{C}$ & 0.849383 & -3.928573 & -2.590407 \\
\hline $\mathrm{H}$ & 0.315271 & -3.809958 & -3.527528 \\
\hline C & 1.514170 & -5.120884 & -2.307278 \\
\hline $\mathrm{H}$ & 1.505527 & -5.933431 & -3.025965 \\
\hline $\mathrm{H}$ & 4.299242 & -1.737223 & 2.002042 \\
\hline $\mathrm{C}$ & 3.618192 & -2.347284 & 2.578316 \\
\hline $\mathrm{C}$ & 4.091502 & -3.055803 & 3.678349 \\
\hline $\mathrm{H}$ & 5.144261 & -3.005281 & 3.936452 \\
\hline $\mathrm{C}$ & 3.214728 & -3.809025 & 4.452059 \\
\hline $\mathrm{H}$ & 3.581653 & -4.356116 & 5.314230 \\
\hline C & 1.862327 & -3.853370 & 4.120905 \\
\hline $\mathrm{H}$ & 1.173638 & -4.439606 & 4.720106 \\
\hline $\mathrm{C}$ & 1.388358 & -3.149132 & 3.019565 \\
\hline $\mathrm{H}$ & 0.333934 & -3.201854 & 2.768506 \\
\hline $\mathrm{Ru}$ & 3.082905 & 0.083798 & -0.223657 \\
\hline C & 2.265259 & -2.385764 & 2.237911 \\
\hline $\mathrm{C}$ & 2.182120 & -5.263438 & -1.096897 \\
\hline $\mathrm{C}$ & 2.187461 & -4.224204 & -0.166496 \\
\hline $\mathrm{H}$ & 2.696939 & -6.189999 & -0.865164 \\
\hline $\mathrm{H}$ & 2.704171 & -4.361964 & 0.776992 \\
\hline $\mathrm{Cl}$ & 3.608213 & 0.884630 & 1.978011 \\
\hline $\mathrm{H}$ & -6.211657 & 1.988472 & 4.101886 \\
\hline $\mathrm{H}$ & -7.704214 & 1.083669 & 3.747251 \\
\hline $\mathrm{H}$ & -7.776443 & 2.857023 & 4.007710 \\
\hline $\mathrm{H}$ & -7.099907 & -3.684849 & -4.339782 \\
\hline $\mathrm{H}$ & -5.691194 & -2.578829 & -4.386998 \\
\hline $\mathrm{H}$ & -7.318495 & -1.925419 & -4.072849 \\
\hline $\mathrm{C}$ & 3.543790 & -0.094181 & -2.512492 \\
\hline $\mathrm{C}$ & 4.255575 & 1.017685 & -1.969523 \\
\hline $\mathrm{C}$ & 5.118991 & 0.867908 & -0.881054 \\
\hline C & 5.368096 & -0.423567 & -0.308922 \\
\hline $\mathrm{C}$ & 4.633045 & -1.508362 & -0.803416 \\
\hline $\mathrm{C}$ & 3.713782 & -1.336280 & -1.879273 \\
\hline $\mathrm{H}$ & 4.109780 & 1.999654 & -2.401154 \\
\hline $\mathrm{H}$ & 5.607644 & 1.737555 & -0.456280 \\
\hline $\mathrm{C}$ & 6.373749 & -0.577155 & 0.790210 \\
\hline $\mathrm{H}$ & 4.752991 & -2.492010 & -0.36243 \\
\hline
\end{tabular}




\begin{tabular}{|c|c|c|c|}
\hline $\mathrm{H}$ & & & 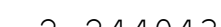 \\
\hline $\mathrm{H}$ & 544 & -0207414 & 0.017625 \\
\hline $\mathrm{H}$ & 6.487254 & -1622232 & $\begin{array}{r}0.44 / 625 \\
1.084359\end{array}$ \\
\hline $\mathrm{H}$ & 6.070365 & 0.011384 & $\begin{array}{l}1.004359 \\
1.661662\end{array}$ \\
\hline $\mathrm{C}$ & 2.775279 & 0.029554 & -3.795715 \\
\hline $\mathrm{H}$ & 3.479751 & -0.047906 & -4.630607 \\
\hline $\mathrm{H}$ & 2.045386 & -0.776167 & -3.903913 \\
\hline $\mathrm{H}$ & 2.274208 & 0.996079 & -3.876198 \\
\hline & & & \\
\hline $2 \mathrm{mc}$ & & & \\
\hline $\mathrm{C}$ & -3.914871 & 0.786550 & -1.667420 \\
\hline $\mathrm{C}$ & -3.344145 & -0.149261 & -2.545969 \\
\hline $\mathrm{C}$ & -3.649571 & -1.524200 & -2.318032 \\
\hline $\mathrm{C}$ & -4.495887 & -1.921844 & -1.278479 \\
\hline $\mathrm{C}$ & -5.139182 & -0.953495 & -0.439893 \\
\hline $\mathrm{C}$ & -4.815885 & 0.395568 & -0.635427 \\
\hline $\mathrm{Ru}$ & -2.814504 & -0.698889 & -0.341103 \\
\hline $\mathrm{Cl}$ & -3.011862 & -2.076029 & 1.616355 \\
\hline $\mathrm{C}$ & -2.564618 & 0.284825 & -3.752858 \\
\hline C & -6.115422 & -1.394142 & 0.606384 \\
\hline $\mathrm{P}$ & -1.957410 & 1.111463 & 0.972474 \\
\hline $\mathrm{C}$ & -2.788538 & 1.361458 & 2.587928 \\
\hline $\mathrm{C}$ & -4.033395 & 0.792323 & 2.860424 \\
\hline $\mathrm{C}$ & -4.698609 & 1.083307 & 4.047802 \\
\hline $\mathrm{C}$ & -4.124858 & 1.946857 & 4.974815 \\
\hline $\mathrm{C}$ & -2.882515 & 2.520298 & 4.711771 \\
\hline $\mathrm{C}$ & -2.216558 & 2.232021 & 3.525830 \\
\hline $\mathrm{C}$ & -0.190187 & 1.249948 & 1.446146 \\
\hline C & 0.324998 & 0.353046 & 2.389639 \\
\hline $\mathrm{C}$ & 1.651796 & 0.442877 & 2.792667 \\
\hline $\mathrm{C}$ & 2.482049 & 1.434877 & 2.271581 \\
\hline $\mathrm{C}$ & 1.965882 & 2.353617 & 1.360986 \\
\hline $\mathrm{C}$ & 0.632870 & 2.262832 & 0.965627 \\
\hline $\mathrm{C}$ & 2.737936 & 3.514957 & 0.795044 \\
\hline 0 & 2.168869 & 4.562233 & 0.513745 \\
\hline $\mathrm{N}$ & 4.072702 & 3.335440 & 0.631158 \\
\hline $\mathrm{C}$ & 4.834063 & 4.413431 & 0.062669 \\
\hline $\mathrm{H}$ & 5.899849 & 4.185047 & 0.151885 \\
\hline $\mathrm{C}$ & 4.526685 & 4.662972 & -1.408520 \\
\hline 0 & 3.996958 & 3.881474 & -2.165339 \\
\hline 0 & 4.966486 & 5.867549 & -1.770640 \\
\hline $\mathrm{C}$ & 4.769985 & 6.216521 & -3.142458 \\
\hline $\mathrm{C}$ & -2.301677 & 2.700121 & 0.109242 \\
\hline $\mathrm{C}$ & -1.647224 & 2.993253 & -1.095747 \\
\hline $\mathrm{C}$ & -1.945122 & 4.149728 & -1.803980 \\
\hline $\mathrm{C}$ & -2.921796 & 5.025297 & -1.329855 \\
\hline $\mathrm{C}$ & -3.584761 & 4.738421 & -0.142739 \\
\hline $\mathrm{C}$ & -3.273994 & 3.585154 & 0.578906 \\
\hline $\mathrm{P}$ & -0.684447 & -1.756615 & -0.699368 \\
\hline $\mathrm{C}$ & -0.851628 & -2.836795 & -2.177933 \\
\hline $\mathrm{C}$ & -1.625726 & -3.995796 & -2.018831 \\
\hline C & -1.849821 & -4.843858 & -3.094923 \\
\hline $\mathrm{C}$ & -1.303495 & -4.546212 & -4.345078 \\
\hline $\mathrm{C}$ & -0.520921 & -3.408689 & -4.503401 \\
\hline $\mathrm{C}$ & -0.287468 & -2.557364 & -3.421002 \\
\hline $\mathrm{C}$ & 0.744150 & -0.666720 & -1.086538 \\
\hline $\mathrm{C}$ & 1.965684 & -0.845756 & -0.44073 \\
\hline
\end{tabular}




\begin{tabular}{|c|c|c|c|}
\hline $\mathrm{C}$ & 3.058033 & -0.032562 & -0.733465 \\
\hline $\mathrm{C}$ & 2.932638 & 1.001608 & -1.657178 \\
\hline$C$ & 1.715192 & 1.191245 & -2.305018 \\
\hline C & 0.632929 & 0.361719 & -2.027195 \\
\hline C & 4.355236 & -0.244228 & -0.014410 \\
\hline 0 & 5.102863 & 0.683568 & 0.301480 \\
\hline $\mathbb{N}$ & 4.659084 & -1.523033 & 0.284836 \\
\hline & 5.861323 & -1.864362 & 1.000321 \\
\hline$H$ & 5.879325 & -1.406330 & 1.994926 \\
\hline$C$ & 5.924257 & -3.371094 & 1.135444 \\
\hline 0 & 5.099098 & -4.125723 & 0.669366 \\
\hline O & 6.995853 & -3.744886 & 1.820637 \\
\hline C & 7.170009 & -5.157883 & 1.990455 \\
\hline$C_{1}$ & 0.127197 & -2.965412 & 0.439576 \\
\hline C & 1.049179 & -3.857481 & -0.136228 \\
\hline 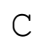 & 1.864322 & -4.652955 & 0.658598 \\
\hline C & 1.768488 & -4.581671 & 2.046822 \\
\hline C & 0.844262 & -3.722309 & 2.626570 \\
\hline $\mathrm{C}$ & 0.031942 & -2.917363 & 1.830123 \\
\hline $\mathrm{H}$ & 4.643988 & 5.341396 & 0.606047 \\
\hline $\mathrm{H}$ & 6.756133 & -1.508402 & 0.477481 \\
\hline $\mathrm{H}$ & 4.107192 & -2.302256 & -0.050776 \\
\hline $\mathrm{H}$ & 4.453610 & 2.390045 & 0.569593 \\
\hline $\mathrm{H}$ & 3.516702 & 1.506964 & 2.590913 \\
\hline $\mathrm{H}$ & 2.035350 & -0.257041 & 3.527802 \\
\hline $\mathrm{H}$ & -0.322764 & -0.391815 & 2.836831 \\
\hline $\mathrm{H}$ & 0.272309 & 3.032186 & 0.296722 \\
\hline $\mathrm{H}$ & 2.064222 & -1.598077 & 0.330356 \\
\hline $\mathrm{H}$ & -0.310941 & 0.519611 & -2.536952 \\
\hline $\mathrm{H}$ & 1.617544 & 2.001737 & -3.019503 \\
\hline $\mathrm{H}$ & 3.755329 & 1.681320 & -1.848992 \\
\hline $\mathrm{H}$ & 1.158328 & -3.916196 & -1.213737 \\
\hline $\mathrm{H}$ & 2.589979 & -5.311939 & 0.195738 \\
\hline $\mathrm{H}$ & 2.409923 & -5.198767 & 2.667153 \\
\hline $\mathrm{H}$ & 0.743028 & -3.672210 & 3.705886 \\
\hline $\mathrm{H}$ & -0.697598 & -2.276246 & 2.297580 \\
\hline $\mathrm{H}$ & -2.053086 & -4.228357 & -1.046265 \\
\hline $\mathrm{H}$ & -2.443664 & -5.741908 & -2.959813 \\
\hline $\mathrm{H}$ & -1.478163 & -5.208938 & -5.186059 \\
\hline $\mathrm{H}$ & -0.074803 & -3.184605 & -5.466823 \\
\hline $\mathrm{H}$ & 0.347367 & -1.687380 & -3.554077 \\
\hline $\mathrm{H}$ & -0.899688 & 2.310216 & -1.482956 \\
\hline $\mathrm{H}$ & -1.413716 & 4.370320 & -2.724064 \\
\hline $\mathrm{H}$ & -3.157490 & 5.929365 & -1.881193 \\
\hline $\mathrm{H}$ & -4.469028 & 0.090951 & 2.162774 \\
\hline $\mathrm{H}$ & -5.659344 & 0.621858 & 4.251476 \\
\hline $\mathrm{H}$ & -4.639781 & 2.169244 & 5.903670 \\
\hline $\mathrm{H}$ & -2.430060 & 3.195764 & 5.430116 \\
\hline $\mathrm{H}$ & -1.253919 & 2.693472 & 3.329842 \\
\hline $\mathrm{H}$ & -4.341632 & 5.417569 & 0.235500 \\
\hline $\mathrm{H}$ & -3.792261 & 3.385674 & 1.510548 \\
\hline $\mathrm{H}$ & 7.253552 & -5.648175 & 1.018774 \\
\hline $\mathrm{H}$ & 6.325239 & -5.581488 & 2.536966 \\
\hline $\mathrm{H}$ & 8.091281 & -5.272485 & 2.557552 \\
\hline $\mathrm{H}$ & 5.226351 & 7.197003 & -3.266119 \\
\hline $\mathrm{H}$ & 3.703021 & 6.260556 & -3.371336 \\
\hline $\mathrm{H}$ & 5.247676 & 5.485372 & -3.79813 \\
\hline
\end{tabular}




\begin{tabular}{|c|c|c|c|}
\hline & & & \\
\hline $\mathrm{H}$ & -3.197803 & -2.277124 & -2.951001 \\
\hline $\mathrm{H}$ & -4.668242 & -2.976824 & -1.097560 \\
\hline $\mathrm{H}$ & -5.243499 & 1.155740 & 0.009157 \\
\hline $\mathrm{H}$ & -3.692836 & 1.837394 & -1.797626 \\
\hline $\mathrm{H}$ & -6.956863 & -1.900370 & 0.123824 \\
\hline $\mathrm{H}$ & -6.504408 & -0.545289 & 1.171627 \\
\hline $\mathrm{H}$ & -5.639005 & -2.095366 & 1.297266 \\
\hline $\mathrm{H}$ & -3.247151 & 0.364457 & -4.605460 \\
\hline $\mathrm{H}$ & -2.111216 & 1.266928 & -3.599239 \\
\hline $\mathrm{H}$ & -1.793592 & -0.442542 & -4.014652 \\
\hline & & & \\
\hline $2 \mathrm{pc}$ & & & \\
\hline $\mathrm{C}$ & 7.982048 & -0.855416 & -3.883173 \\
\hline 0 & 7.777011 & -1.521126 & -2.631614 \\
\hline $\mathrm{C}$ & 8.176393 & 0.927499 & 3.598130 \\
\hline 0 & 7.819695 & 1.564299 & 2.365901 \\
\hline $\mathrm{C}$ & 6.803233 & 1.029706 & 1.706901 \\
\hline C & 6.540179 & 1.768138 & 0.401147 \\
\hline $\mathrm{C}$ & 6.798620 & -1.046862 & -1.875537 \\
\hline $\mathrm{C}$ & 6.686360 & -1.815278 & -0.564302 \\
\hline 0 & 6.113164 & -0.089167 & -2.176193 \\
\hline 0 & 6.197360 & 0.044186 & 2.081178 \\
\hline $\mathrm{N}$ & 5.360164 & -1.719206 & -0.019868 \\
\hline $\mathrm{C}$ & 4.362077 & -2.260736 & -0.760211 \\
\hline $\mathrm{N}$ & 5.177388 & 1.608230 & -0.027123 \\
\hline $\mathrm{C}$ & 4.225586 & 2.131555 & 0.783781 \\
\hline 0 & 4.612265 & -2.879746 & -1.788477 \\
\hline 0 & 4.539890 & 2.747306 & 1.796963 \\
\hline $\mathrm{H}$ & 7.198671 & 1.366319 & -0.372381 \\
\hline $\mathrm{H}$ & 6.785152 & 2.821619 & 0.550651 \\
\hline $\mathrm{H}$ & 6.964285 & -2.854519 & -0.751143 \\
\hline $\mathrm{H}$ & 7.392721 & -1.393288 & 0.155447 \\
\hline $\mathrm{H}$ & 5.229636 & -1.102742 & 0.774739 \\
\hline $\mathrm{H}$ & 5.008256 & 0.976222 & -0.801316 \\
\hline $\mathrm{C}$ & 2.774940 & 1.941843 & 0.436863 \\
\hline $\mathrm{C}$ & 2.279822 & 1.403547 & -0.753871 \\
\hline $\mathrm{H}$ & 2.949025 & 1.070645 & -1.541573 \\
\hline $\mathrm{C}$ & 0.908222 & 1.287818 & -0.953328 \\
\hline $\mathrm{H}$ & 0.536839 & 0.867644 & -1.881915 \\
\hline $\mathrm{C}$ & 0.006921 & 1.721812 & 0.030031 \\
\hline $\mathrm{H}$ & -0.146393 & 2.658851 & 1.976789 \\
\hline $\mathrm{C}$ & 0.510059 & 2.266066 & 1.213543 \\
\hline $\mathrm{P}$ & -1.786220 & 1.738223 & -0.381662 \\
\hline $\mathrm{C}$ & 1.878283 & 2.364379 & 1.413839 \\
\hline $\mathrm{H}$ & 2.279933 & 2.798307 & 2.322285 \\
\hline $\mathrm{C}$ & 2.937518 & -2.073237 & -0.317385 \\
\hline $\mathrm{C}$ & 1.979459 & -2.618319 & -1.169857 \\
\hline $\mathrm{H}$ & 2.322002 & -3.143561 & -2.053691 \\
\hline $\mathrm{C}$ & 0.630227 & -2.494119 & -0.892311 \\
\hline $\mathrm{C}$ & 0.198734 & -1.804806 & 0.245467 \\
\hline $\mathrm{P}$ & -1.602339 & -1.678941 & 0.595532 \\
\hline $\mathrm{H}$ & -0.075906 & -2.936779 & -1.578000 \\
\hline $\mathrm{C}$ & 1.160260 & -1.244656 & 1.094872 \\
\hline $\mathrm{H}$ & 0.864412 & -0.678697 & 1.970867 \\
\hline C & 2.519270 & -1.388251 & 0.823642 \\
\hline $\mathrm{H}$ & 3.233046 & -0.941989 & 1.510177 \\
\hline $\mathrm{C}$ & -1.658418 & -1.541309 & 2.42103 \\
\hline
\end{tabular}




\begin{tabular}{|c|c|c|c|}
\hline $\mathrm{C}$ & -2.227195 & -3.391654 & 0.292480 \\
\hline $\mathrm{C}$ & -2.683676 & -4.205453 & 1.332421 \\
\hline $\mathrm{H}$ & -2.631698 & -3.869487 & 2.361396 \\
\hline $\mathrm{C}$ & -3.223773 & -5.463271 & 1.063393 \\
\hline $\mathrm{H}$ & -3.575084 & -6.079356 & 1.884556 \\
\hline $\mathrm{C}$ & -3.307331 & -5.927133 & -0.243547 \\
\hline $\mathrm{H}$ & -3.722518 & -6.908043 & -0.449285 \\
\hline $\mathrm{C}$ & -2.862942 & -5.118585 & -1.288919 \\
\hline $\mathrm{H}$ & -2.932814 & -5.466119 & -2.314968 \\
\hline $\mathrm{C}$ & -2.340797 & -3.858408 & -1.026585 \\
\hline $\mathrm{H}$ & -2.065806 & -3.214915 & -1.856561 \\
\hline $\mathrm{C}$ & -1.734081 & 2.838813 & -1.853993 \\
\hline $\mathrm{C}$ & -1.021663 & 4.042904 & -1.747163 \\
\hline $\mathrm{H}$ & -0.498237 & 4.287230 & -0.827386 \\
\hline $\mathrm{C}$ & -0.981957 & 4.932891 & -2.811656 \\
\hline $\mathrm{H}$ & -0.432263 & 5.863063 & -2.713981 \\
\hline $\mathrm{C}$ & -1.642641 & 4.629404 & -4.001829 \\
\hline $\mathrm{H}$ & -1.608279 & 5.324985 & -4.833845 \\
\hline $\mathrm{Ru}$ & -3.056655 & -0.241485 & -0.662455 \\
\hline $\mathrm{C}$ & -2.561847 & 2.830440 & 0.87988 \\
\hline $\mathrm{C}$ & -2.330875 & 3.428617 & -4.123016 \\
\hline $\mathrm{C}$ & -2.374308 & 2.535768 & -3.053513 \\
\hline $\mathrm{H}$ & -2.824352 & 3.174446 & -5.055318 \\
\hline $\mathrm{H}$ & -2.861631 & 1.579070 & -3.173425 \\
\hline $\mathrm{H}$ & 7.091222 & -0.949147 & -4.506780 \\
\hline $\mathrm{H}$ & 8.198850 & 0.202507 & -3.721561 \\
\hline $\mathrm{H}$ & 8.830074 & -1.355721 & -4.346334 \\
\hline $\mathrm{H}$ & 9.050020 & 1.463676 & 3.963178 \\
\hline $\mathrm{H}$ & 7.353509 & 1.003231 & 4.311208 \\
\hline $\mathrm{H}$ & 8.411467 & -0.125456 & 3.430240 \\
\hline $\mathrm{C}$ & -5.023503 & 0.421415 & 0.398010 \\
\hline $\mathrm{C}$ & -4.856514 & -0.976901 & 0.486168 \\
\hline $\mathrm{C}$ & -4.780116 & -1.796201 & -0.676743 \\
\hline $\mathrm{C}$ & -4.753597 & -1.226432 & -1.952896 \\
\hline C & -4.791320 & 0.201551 & -2.048741 \\
\hline $\mathrm{C}$ & -4.938575 & 1.000140 & -0.903951 \\
\hline $\mathrm{H}$ & -4.863070 & -1.454194 & 1.460716 \\
\hline $\mathrm{H}$ & -4.698636 & -2.869635 & -0.564684 \\
\hline C & -4.657572 & -2.063454 & -3.190948 \\
\hline $\mathrm{H}$ & -4.724105 & 0.665534 & -3.025038 \\
\hline $\mathrm{H}$ & -4.982472 & 2.079903 & -1.006608 \\
\hline $\mathrm{H}$ & -5.592609 & -1.986710 & -3.754516 \\
\hline $\mathrm{H}$ & -3.838513 & -1.711007 & -3.823345 \\
\hline $\mathrm{H}$ & -4.483315 & -3.111925 & -2.944767 \\
\hline $\mathrm{C}$ & -5.434638 & 1.240769 & 1.586025 \\
\hline $\mathrm{H}$ & -6.523590 & 1.167941 & 1.680506 \\
\hline $\mathrm{H}$ & -5.177601 & 2.292585 & 1.467588 \\
\hline $\mathrm{H}$ & -5.002232 & 0.872395 & 2.517218 \\
\hline $\mathrm{C}$ & -2.467610 & 2.522261 & 2.242566 \\
\hline C & -3.083938 & 3.319703 & 3.200055 \\
\hline C & -3.829773 & 4.429445 & 2.810685 \\
\hline $\mathrm{C}$ & -3.951171 & 4.733708 & 1.458146 \\
\hline $\mathrm{C}$ & -3.318584 & 3.944569 & 0.499114 \\
\hline $\mathrm{H}$ & -1.905895 & 1.654963 & 2.568228 \\
\hline $\mathrm{H}$ & -2.978020 & 3.073187 & 4.251712 \\
\hline $\mathrm{H}$ & -4.311375 & 5.053689 & 3.555855 \\
\hline $\mathrm{H}$ & -4.530224 & 5.595512 & 1.1430 \\
\hline
\end{tabular}




\begin{tabular}{|c|c|c|c|}
\hline & & & \\
\hline $\mathrm{H}$ & -3.410943 & 4.210944 & -0.548258 \\
\hline C & -2.584832 & -0.698671 & 3.030158 \\
\hline $\mathrm{C}$ & -2.683319 & -0.620157 & 4.417279 \\
\hline C & -1.851894 & -1.399377 & 5.212968 \\
\hline $\mathrm{C}$ & -0.925107 & -2.254687 & 4.617089 \\
\hline C & -0.827353 & -2.328281 & 3.232940 \\
\hline $\mathrm{H}$ & -3.406365 & 0.051301 & 4.870254 \\
\hline $\mathrm{H}$ & -1.923062 & -1.343275 & 6.294137 \\
\hline $\mathrm{H}$ & -0.276604 & -2.868071 & 5.233561 \\
\hline $\mathrm{H}$ & -0.104551 & -3.000942 & 2.782359 \\
\hline $\mathrm{Cl}$ & -1.598272 & -0.686870 & -2.524816 \\
\hline $\mathrm{H}$ & -3.220838 & -0.085106 & 2.407719 \\
\hline & & & \\
\hline & & & \\
\hline $\mathrm{C}$ & -2.120618 & -3.395252 & 2.210510 \\
\hline $\mathrm{C}$ & -2.590780 & -2.192223 & 1.661000 \\
\hline $\mathrm{C}$ & -3.453473 & -1.401461 & 2.413181 \\
\hline $\mathrm{C}$ & -3.851699 & -1.790465 & 3.691019 \\
\hline $\mathrm{C}$ & -3.386323 & -2.985720 & 4.225204 \\
\hline $\mathrm{C}$ & -2.518981 & -3.787407 & 3.481366 \\
\hline $\mathrm{P}$ & -2.072040 & -1.697299 & -0.026058 \\
\hline $\mathrm{Ru}$ & -2.967597 & 0.327510 & -0.910112 \\
\hline $\mathrm{Cl}$ & -1.268675 & 0.126092 & -2.608907 \\
\hline $\mathrm{C}$ & -0.265759 & -1.989139 & 0.070756 \\
\hline $\mathrm{C}$ & 0.468816 & -2.490375 & -1.007032 \\
\hline $\mathrm{C}$ & 1.823672 & -2.756702 & -0.865485 \\
\hline $\mathrm{C}$ & 2.473739 & -2.515464 & 0.342862 \\
\hline $\mathrm{C}$ & 1.751360 & -1.992334 & 1.415180 \\
\hline $\mathrm{C}$ & 0.394494 & -1.731298 & 1.278246 \\
\hline C & 3.927107 & -2.891253 & 0.420116 \\
\hline 0 & 4.410866 & -3.678927 & -0.380071 \\
\hline $\mathrm{N}$ & 4.656335 & -2.338472 & 1.427995 \\
\hline $\mathrm{C}$ & 6.073950 & -2.577019 & 1.419879 \\
\hline $\mathrm{C}$ & 6.757901 & -1.927646 & 0.224836 \\
\hline 0 & 6.289333 & -1.032921 & -0.442662 \\
\hline 0 & 7.971735 & -2.444353 & 0.043944 \\
\hline $\mathrm{C}$ & 8.740888 & -1.869078 & -1.014825 \\
\hline $\mathrm{C}$ & -2.769621 & -3.044694 & -1.076382 \\
\hline $\mathrm{C}$ & -3.689324 & -3.962407 & -0.557541 \\
\hline $\mathrm{C}$ & -4.305734 & -4.895975 & -1.390055 \\
\hline $\mathrm{C}$ & -4.009656 & -4.930502 & -2.747579 \\
\hline $\mathrm{C}$ & -3.100011 & -4.015674 & -3.274877 \\
\hline $\mathrm{C}$ & -2.494202 & -3.073850 & -2.452047 \\
\hline $\mathrm{P}$ & -1.482667 & 1.812349 & 0.209891 \\
\hline $\mathrm{C}$ & -2.241798 & 2.378963 & 1.781514 \\
\hline $\mathrm{C}$ & -2.251138 & 1.517183 & 2.884840 \\
\hline $\mathrm{C}$ & -2.885085 & 1.880048 & 4.067859 \\
\hline $\mathrm{C}$ & -3.529164 & 3.111657 & 4.165743 \\
\hline $\mathrm{C}$ & -3.534755 & 3.973265 & 3.072826 \\
\hline $\mathrm{C}$ & -2.896193 & 3.610836 & 1.888176 \\
\hline C & 0.261030 & 1.411570 & 0.649709 \\
\hline $\mathrm{C}$ & 1.133947 & 1.070925 & -0.391684 \\
\hline $\mathrm{C}$ & 2.498495 & 0.959822 & -0.158997 \\
\hline $\mathrm{C}$ & 3.008671 & 1.164074 & 1.123189 \\
\hline $\mathrm{C}$ & 2.140710 & 1.457911 & 2.171115 \\
\hline $\mathrm{C}$ & 0.780249 & 1.609165 & 1.933012 \\
\hline $\mathrm{C}$ & 4.475305 & 1.067977 & 1.42506 \\
\hline
\end{tabular}




\begin{tabular}{|c|c|c|c|}
\hline O & 4.887107 & 0.402922 & 2.376386 \\
\hline $\mathrm{N}$ & 5.270051 & 1.793971 & 0.619447 \\
\hline $\mathrm{C}$ & 6.707069 & 1.720292 & 0.670937 \\
\hline $\mathrm{C}$ & 7.238638 & 1.873448 & -0.737979 \\
\hline 0 & 6.561253 & 2.217911 & -1.677758 \\
\hline 0 & 8.547239 & 1.631526 & -0.794588 \\
\hline $\mathrm{C}$ & 9.144859 & 1.783599 & -2.087750 \\
\hline $\mathrm{C}$ & -1.165864 & 3.375079 & -0.708248 \\
\hline $\mathrm{C}$ & -0.523090 & 4.428534 & -0.040791 \\
\hline $\mathrm{C}$ & -0.223119 & 5.606859 & -0.710522 \\
\hline $\mathrm{C}$ & -0.540231 & 5.743622 & -2.061661 \\
\hline $\mathrm{C}$ & -1.144244 & 4.691424 & -2.738738 \\
\hline $\mathrm{C}$ & -1.452231 & 3.509800 & -2.066482 \\
\hline C & -5.009799 & 0.883486 & 0.068278 \\
\hline $\mathrm{C}$ & -5.039755 & -0.430447 & -0.451095 \\
\hline $\mathrm{C}$ & -4.799300 & -0.702335 & -1.825857 \\
\hline $\mathrm{C}$ & -4.451447 & 0.333800 & -2.707317 \\
\hline $\mathrm{C}$ & -4.311769 & 1.651047 & -2.176413 \\
\hline $\mathrm{C}$ & -4.575971 & 1.911248 & -0.818960 \\
\hline $\mathrm{C}$ & -5.532585 & 1.212202 & 1.437780 \\
\hline $\mathrm{C}$ & -4.201979 & 0.060443 & -4.158166 \\
\hline $\mathrm{H}$ & 7.133042 & 2.515238 & 1.295386 \\
\hline $\mathrm{H}$ & 7.014903 & 0.764158 & 1.092844 \\
\hline $\mathrm{H}$ & 6.284076 & -3.647746 & 1.402171 \\
\hline $\mathrm{H}$ & 6.508931 & -2.154089 & 2.329334 \\
\hline $\mathrm{H}$ & 4.365964 & -1.468588 & 1.862743 \\
\hline $\mathrm{H}$ & 4.887267 & 2.238690 & -0.204869 \\
\hline $\mathrm{H}$ & 3.165485 & 0.691577 & -0.973373 \\
\hline $\mathrm{H}$ & 0.747830 & 0.900268 & -1.389119 \\
\hline $\mathrm{H}$ & 0.141680 & 1.903198 & 2.756488 \\
\hline $\mathrm{H}$ & 2.541707 & 1.596235 & 3.169803 \\
\hline $\mathrm{H}$ & 2.396465 & -3.179247 & -1.683384 \\
\hline $\mathrm{H}$ & -0.006170 & -2.697072 & -1.956246 \\
\hline $\mathrm{H}$ & -0.148640 & -1.344017 & 2.133270 \\
\hline $\mathrm{H}$ & 2.232054 & -1.809013 & 2.370470 \\
\hline $\mathrm{H}$ & -3.939465 & -3.958586 & 0.497028 \\
\hline $\mathrm{H}$ & -5.016657 & -5.599229 & -0.968806 \\
\hline $\mathrm{H}$ & -4.485512 & -5.661695 & -3.392534 \\
\hline $\mathrm{H}$ & -2.865685 & -4.028570 & -4.334562 \\
\hline $\mathrm{H}$ & -1.840648 & -2.329170 & -2.891835 \\
\hline $\mathrm{H}$ & -0.252481 & 4.326903 & 1.006082 \\
\hline $\mathrm{H}$ & 0.267249 & 6.417001 & -0.180979 \\
\hline $\mathrm{H}$ & -0.300722 & 6.663375 & -2.585022 \\
\hline $\mathrm{H}$ & -1.362510 & 4.779140 & -3.798042 \\
\hline $\mathrm{H}$ & -1.863639 & 2.671577 & -2.612965 \\
\hline $\mathrm{H}$ & 8.218535 & -1.980735 & -1.967383 \\
\hline $\mathrm{H}$ & 8.916931 & -0.808797 & -0.819664 \\
\hline $\mathrm{H}$ & 9.680806 & -2.418149 & -1.028218 \\
\hline $\mathrm{H}$ & 10.206966 & 1.594270 & -1.942993 \\
\hline $\mathrm{H}$ & 8.715698 & 1.063726 & -2.788175 \\
\hline $\mathrm{H}$ & 8.982085 & 2.793745 & -2.467963 \\
\hline $\mathrm{H}$ & -5.297314 & -1.253778 & 0.207979 \\
\hline $\mathrm{H}$ & -4.851800 & -1.722050 & -2.187436 \\
\hline $\mathrm{H}$ & -4.000544 & 2.458498 & -2.826767 \\
\hline $\mathrm{H}$ & -4.460162 & 2.921340 & -0.438332 \\
\hline $\mathrm{H}$ & -5.122532 & 0.258231 & -4.717688 \\
\hline $\mathrm{H}$ & -3.408638 & 0.699158 & -4.54848 \\
\hline
\end{tabular}




\begin{tabular}{|c|c|c|c|}
\hline$H$ & 301601 & & $7100 r$ \\
\hline & -3.916410 & -0.981129 & -4.314269 \\
\hline $\mathrm{H}$ & -6.462941 & 1.777773 & 1.324818 \\
\hline $\mathrm{H}$ & -4.838785 & 1.825579 & 2.012771 \\
\hline $\mathrm{H}$ & -5.766903 & 0.308826 & 2.003077 \\
\hline $\mathrm{H}$ & -1.759078 & 0.552152 & 2.826005 \\
\hline $\mathrm{H}$ & -2.872961 & 1.197476 & 4.911482 \\
\hline $\mathrm{H}$ & -4.021787 & 3.398032 & 5.088861 \\
\hline $\mathrm{H}$ & -4.032294 & 4.935025 & 3.140035 \\
\hline $\mathrm{H}$ & -2.900304 & 4.303272 & 1.052934 \\
\hline $\mathrm{H}$ & -4.516339 & -1.152960 & 4.265989 \\
\hline $\mathrm{H}$ & -3.691090 & -3.294447 & 5.219800 \\
\hline $\mathrm{H}$ & -2.148531 & -4.718693 & 3.896480 \\
\hline $\mathrm{H}$ & -1.441893 & -4.024262 & 1.642819 \\
\hline $\mathrm{H}$ & -3.789088 & -0.457323 & 2.008892 \\
\hline & & & \\
\hline & $\operatorname{Im}$ & & \\
\hline C & -1.2694149208 & 1.6007465424 & -2.5479859035 \\
\hline & -1.7651697229 & 0.3932947865 & -3.06532 \\
\hline & -3.1628524939 & 0.1323517551 & -2.9195661441 \\
\hline & -4.0099252157 & 1.0827199915 & -2.3453536583 \\
\hline & -3.5215205464 & 2.3490416538 & -1.8832616995 \\
\hline $\mathrm{C}$ & -2.1406828686 & 2.5722421121 & -1.9715517525 \\
\hline $\mathrm{Ru}$ & -2.4172748592 & 0.6388729289 & -0.8091311208 \\
\hline $\mathrm{Cl}$ & -4.384117591 & 0.4116292177 & 0.581269675 \\
\hline 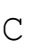 & -0.8881353449 & -0.5264912419 & -3.8621758861 \\
\hline & -4.4737386132 & 3.3674547592 & -1.332915963 \\
\hline $\mathrm{P}$ & -1.0081845804 & 1.5546176702 & 0.9440704232 \\
\hline C & -1.8618656578 & 2.4685555634 & 2.2849805387 \\
\hline $\mathrm{C}$ & -3.1191149072 & 3.0315754826 & 2.06085 \\
\hline C & -3.7052441979 & 3.8556419525 & 3.01832 \\
\hline$a^{2}$ & -3.0403503913 & 4.1241981187 & 4.2096531592 \\
\hline $\mathrm{C}$ & -1.7805945305 & 3.5738541744 & 4.4387965837 \\
\hline C & -1.1905870315 & 2.757310845 & 3.4817090193 \\
\hline C & 0.1235186205 & 0.454340342 & 1.9002759993 \\
\hline $\mathrm{C}$ & -0.4389474748 & -0.2581211376 & 2.9631339617 \\
\hline C & 0.3041003043 & -1.1887531327 & 3.6769711211 \\
\hline $\mathrm{C}$ & 1.6320802364 & -1.4079406487 & 3.3465187088 \\
\hline C & 2.2308429411 & -0.6705773026 & 2.3238579784 \\
\hline C & 1.4776750445 & 0.2618717159 & 1.6067418357 \\
\hline $\mathrm{C}$ & 3.687003115 & -0.9642413359 & 2.0810458783 \\
\hline 0 & 4.2208630907 & -1.937118173 & 2.6068552505 \\
\hline $\mathrm{N}$ & 4.400935983 & -0.1225332714 & 1.29501725 \\
\hline $\mathrm{C}$ & 5.8483605425 & -0.236677494 & 1.2507975834 \\
\hline $\mathrm{H}$ & 6.1666153999 & 0.4175669329 & 0.431841308 \\
\hline$C^{\prime}$ & 6.2394220 & -1.6462580147 & 0.8057347311 \\
\hline 0 & 5.783488238 & -2.1772472787 & -0.1949240759 \\
\hline 0 & 7.1770382245 & -2.1955147988 & 1.5495726176 \\
\hline $\mathrm{C}$ & 7.5785766035 & -3.5214445411 & 1.1862045818 \\
\hline C & 0.0778829937 & 2.8774808419 & 0.256205554 \\
\hline C & 1.0321477297 & 2.5655382357 & -0.7168501898 \\
\hline C & 1.742684798 & 3.5673059761 & -1.3677457498 \\
\hline C & 1.527173606 & 4.9024528317 & -1.041967967 \\
\hline$C$ & 0.6040592674 & 5.2249420171 & -0.051208138 \\
\hline C & -0.1191129328 & 4.2233667189 & 0.5926598514 \\
\hline P & -1.9429813925 & -1.6363745599 & -0.221402084 \\
\hline C & -2.6402333172 & -2.7384537894 & -1.5136034348 \\
\hline$a_{1}+2$ & -4.0357388444 & -2.767386973 & -1.6449104804 \\
\hline
\end{tabular}




\begin{tabular}{|c|c|c|c|}
\hline & -4.6293741828 & -3.580957748 & -2.6014744184 \\
\hline & -3.8388642186 & -4.3753960698 & -3.4339080818 \\
\hline & -2.4558293564 & -4.3594025924 & -3.2976521744 \\
\hline & -1.8548286506 & -3.5461874747 & -2.3355316706 \\
\hline & -0.1516203341 & -2.0460019841 & 63672595 \\
\hline & 0.6715433984 & -1.2585818064 & -1.0339700705 \\
\hline & 2.0317309468 & -1.515 & -1.17 \\
\hline & 2.5859355203 & -2.6075926132 & -0.5009410346 \\
\hline & 1.7798273593 & -3.3864972202 & 0.3218258187 \\
\hline & 0.4231491993 & -3.1097918531 & 0.4655216902 \\
\hline & 2.812462642 & -0.5178620616 & 936118 \\
\hline & 7396 & 0.3709409662 & -2.6 \\
\hline & 2473447 & -0.6165507501 & -1.9442135827 \\
\hline & 5.0039096039 & 0.3699895361 & -2.574976073 \\
\hline & 4.8702765194 & 0.4222556492 & -4.0982075228 \\
\hline & 72225905 & 77041684 & -1 \\
\hline & 4.4388021142 & 1.8845005034 & -0.7 \\
\hline & 659568 & 2.7244672366 & -2.6317009622 \\
\hline & 697775 & 4.0062409708 & -1.9960700091 \\
\hline & -2.6069713331 & -2.4890289678 & 1.2696343019 \\
\hline & -2.6203110399 & -3.8928866586 & 1.2670187697 \\
\hline & -3.0478264634 & -4.6018273188 & 72325 \\
\hline & -3.4900705891 & -3.9210979403 & 3.5146680911 \\
\hline & -3.5166401584 & -2.5311459161 & 3.5133658818 \\
\hline & -3 & -1.8206427572 & 68876 \\
\hline & 6.5117871226 & 0.2234565273 & 2.5469933067 \\
\hline & 6.0318656607 & 0.0657568489 & $-2 \cdot 33$ \\
\hline & 116 & -1.2428132235 & 12392 \\
\hline & 4.0039946241 & 0.6636465211 & 0.7977340379 \\
\hline & 2.2369250976 & -2.1359345665 & 3.874752274 \\
\hline & -0.1607909299 & -1.7374768402 & 4.4890113497 \\
\hline & $-1.46232 \varepsilon$ & -0.0704571568 & 23885 \\
\hline & 1.940547956 & 0.8324642056 & 0.8106440468 \\
\hline & 0.2663780798 & -0.3927467517 & -1.5360935045 \\
\hline & -0.1737807102 & -3.7173252449 & 1.1350770202 \\
\hline & 2.2213917898 & -4.2062711875 & 0.8773821275 \\
\hline & 3.64127955 & -2.845420428 & 0.5740794796 \\
\hline & -2.3069650725 & -4.4427801967 & 0.3852738228 \\
\hline & -3.0460594522 & -5.6866205675 & 2.3616917273 \\
\hline & -3.832144869 & -4.4732270934 & 4.3839289665 \\
\hline & 9887084 & -1.9908888869 & 4.3754601189 \\
\hline & -3.1670457634 & -0.7440237117 & 2.390344348 \\
\hline & -4.6507938401 & -2.1484052716 & 2646706 \\
\hline & -5.7 & 21559809 & 688825 \\
\hline & -4.304328261 & -5.0117837499 & -4.1792502125 \\
\hline & -1.8375329592 & -4.9847948922 & -3.9332649737 \\
\hline & -0.7743480187 & -3.5509756298 & -2.2281137587 \\
\hline & 1.2122652209 & 1.5433048972 & -1.0266028213 \\
\hline & 2.444918515 & 3.2837947043 & -2.1437197655 \\
\hline & 3331527 & 5.6888813332 & -1.5578243206 \\
\hline & -3.650189514 & 2.8088309438 & 1.1463683796 \\
\hline & -4.6870237215 & 4.2787324884 & 2.8333647177 \\
\hline & -3.4995029972 & 4.7613598639 & 4.9584256767 \\
\hline & -1.2531278174 & 3.7842520448 & 5.3632674612 \\
\hline & -0.2013251396 & 2.3530770303 & 3.6670931196 \\
\hline & 0.4333307337 & 6.2621771236 & 0.2178213975 \\
\hline & -0.8 & 4.50214 & \\
\hline
\end{tabular}




\begin{tabular}{|c|c|c|c|}
\hline & 6.1640404781 & 3.9806640553 & -1.2190470886 \\
\hline & 4.4440815435 & 4.2854126992 & -1.5449083042 \\
\hline & 5.6771000327 & 4.7035038559 & -2.7829438071 \\
\hline & 8.3428149717 & -3.8019903837 & 1.9077136958 \\
\hline & 7.9809843976 & -3.5335057817 & 0.1715457587 \\
\hline & 6.7243794482 & -4.1978098686 & 1.2 \\
\hline & -3.5664608032 & 0861 & 701 \\
\hline & -5.056601781 & 0.84269414 & .1964 \\
\hline & -1.7177435352 & 3.4916832702 & -1.5814464635 \\
\hline & -0.2108026182 & 1.8077074608 & -2.630791959 \\
\hline & 505289982 & 71682704 & 5372 \\
\hline & -3.9423 & 4.1785 & $-0 . \varepsilon-\gamma-\gamma$ \\
\hline & -5.16 & 2.89 & -0 . \\
\hline & -1.1094343412 & -0.3583975445 & -4.9219674014 \\
\hline & 0.1713660138 & -0.320005375 & -3.6971358274 \\
\hline & -1.1004924019 & -1.5743561159 & -3 \\
\hline & 7.5 & 0.184462488 & 2.460 \\
\hline & 6.2 & 1.2531 & 2.7549 \\
\hline & 6.20 & 18976 & 3.3762126885 \\
\hline & 5.6 & 1.09 & -4.5203954713 \\
\hline & 5.0 & -0.5802479784 & -4.5017190912 \\
\hline & 3.87 & 0.7681557825 & -4.3839231745 \\
\hline & & & \\
\hline & om & & \\
\hline & -1.2520210498 & -1.1083998529 & 2.8007200671 \\
\hline & -1.5 & 0.22560 & 3.1137034501 \\
\hline & -2.94286 & 0.6082524411 & 3.0941013749 \\
\hline & -3.9 & -0.327 & 2.81 \\
\hline & -3.632077 & -1.7023175954 & 2.54277933 \\
\hline & -2.2780311939 & -2.0629031036 & 2.5361019716 \\
\hline & -2.5282319766 & -0.349716165 & 1.0528951722 \\
\hline$\perp$ & -4.643 & -0.1004265718 & 422095 \\
\hline & -0.504 & 1.17 & 3.57 \\
\hline & -4.72636716 & -2.6930693968 & 2.281 \\
\hline & -1.4590778211 & -1.7286542881 & -0.6432386347 \\
\hline & -2.5791961389 & -2.5376406495 & -1.8454509233 \\
\hline & -3.861876 & -2.919253904 & -1.4408383287 \\
\hline & -4 & 6724 & 2983 \\
\hline & -4.2 & 335 & -3 \\
\hline & -2.942606 & -3.660 & -3.9596343898 \\
\hline & -2.1203604762 & -2.9282232317 & -3.1099499167 \\
\hline & -0.1444 & -1.024761119 & -1.7334993578 \\
\hline & -0.527 & 67419 & 340927 \\
\hline & 0.4143874 & 0.474 & -3.562 \\
\hline & 1.7 & 0.27 & -3.32 \\
\hline & 2.1672072 & -0.6163836882 & -2.3326763037 \\
\hline & 1.2138219465 & -1.2801957554 & -1.5599107855 \\
\hline 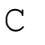 & & -0 . & 12461 \\
\hline O & $3.9^{7}-(x)$ & -2.0 & -1.6 \\
\hline & 4.4 & 0.11 & -2.244 \\
\hline & 5.8 & -0.0 & -2.0347613487 \\
\hline$C$ & 6.6047936502 & -0.5958515374 & -3.2662621145 \\
\hline C & 6.431096405 & 1.3063266431 & -1.5660047866 \\
\hline O & 5.7211584086 & 2.2359167766 & -1.2311864727 \\
\hline 0 & 7.7 & 97367 & -1.55 \\
\hline $\mathrm{C}$ & 8.35 & 2.4990712166 & -0.9772295879 \\
\hline & -0.626279 & & 0.1324832549 \\
\hline
\end{tabular}




\begin{tabular}{|c|c|c|c|}
\hline C & 0.475089019 & -3.0045643306 & 0.9827903755 \\
\hline & 1.0562669926 & -4.0912822109 & 1.6260228572 \\
\hline & 0.5490757513 & -5.374140574 & 1.4346424307 \\
\hline & -0.5373480348 & -5.5614248657 & 0.587893214 \\
\hline & -1.1211952251 & -4.4753552681 & -0.062002133 \\
\hline & -1.9426984725 & 1.7200563865 & 0.0074687605 \\
\hline & -2.4583678104 & 3.1035093967 & 1.10001 \\
\hline & -3.8335489252 & 3.265455218 & 1.3222405474 \\
\hline & -4.2906826178 & 4.2848258222 & 2.1473490258 \\
\hline & -3.3844669321 & 5.1542204283 & 2.7570926501 \\
\hline & -2.0216755682 & 5.0043438154 & 2.5299726505 \\
\hline & -1.5572411695 & 3.9840130512 & 672933 \\
\hline & -0.1310130519 & 1.976912351 & -0.17 \\
\hline & 0.6990857796 & 1.1331445027 & 0.5499797209 \\
\hline & 2.0806797218 & 1.2943607898 & 0.5749453012 \\
\hline $\mathrm{C}$ & 2.6566257538 & 2.3351857708 & -0.1518751207 \\
\hline $\mathrm{C}$ & 1.8383560921 & 3.1733342263 & -0.9076 \\
\hline $\mathrm{C}$ & 0.4578511959 & 2.9997752685 & -0.9274 \\
\hline C & 2.8278066577 & 0.2804566148 & 1.3912393944 \\
\hline O & 2.2222938444 & -0.4492368788 & 2.1863258141 \\
\hline $\mathrm{N}$ & 4.1569772389 & 0.1814196406 & 1.2127377148 \\
\hline $\mathrm{C}$ & 4.9366846873 & -0.7748603309 & 1.97656 \\
\hline$C$ & 4.7 & -2.216 & 1.4 \\
\hline $\mathrm{C}$ & 6.3927373005 & -0.3592216363 & 1.888631392 \\
\hline 0 & 6.8212162607 & 0.4657215712 & 1.1092891453 \\
\hline 0 & 7.1415062712 & -1.0291443703 & 2.7538039119 \\
\hline $\mathrm{C}$ & 8.5462636189 & -0.7456949603 & 2.7184987407 \\
\hline r & -2.6931399549 & 2.2 & -1.57 \\
\hline $\mathrm{C}$ & -2.6650471911 & 3.6666146044 & -1.860 \\
\hline $\mathrm{C}$ & -3.1749787211 & 4.1542311759 & -3.058813303 \\
\hline $\mathrm{C}$ & -3.7411201148 & 3.2804632554 & -3.9821889334 \\
\hline $\mathrm{C}$ & -3.8090225236 & 1.9217250724 & -3.691 \\
\hline $\mathrm{C}$ & -3.2959006146 & 1.43272 & -2.493 \\
\hline$H$ & 6.04 & -0.7273606096 & -1.197 \\
\hline $\mathrm{H}$ & 4.6280014219 & -0.7162630659 & 3.0253645822 \\
\hline $\mathrm{H}$ & 4.656932233 & 0.7698323425 & 0.5568809989 \\
\hline $\mathrm{H}$ & 4.1108238797 & 1.0582769384 & -2.3052256404 \\
\hline $\mathrm{H}$ & 2.510 & 0.7678933217 & -3.9 \\
\hline & 0.08 & 1.129624775 & -4.36149 \\
\hline $\mathrm{H}$ & -1.5725029565 & -0.0006410035 & -2.9809743294 \\
\hline $\mathrm{H}$ & 1.5800871077 & -1.9932397654 & -0.8337999576 \\
\hline $\mathrm{H}$ & 0.2802197369 & 0.3033092847 & 1.1007793006 \\
\hline $\mathrm{H}$ & -0.1513741288 & 3.6599749419 & -1.5337946541 \\
\hline & 2.2853151062 & 3.9744968094 & -1.4868907785 \\
\hline $\mathrm{H}$ & 3.7289548594 & 2.5043402261 & -0.1620485785 \\
\hline $\mathrm{H}$ & -2.2688330712 & 4.3707864461 & -1.1354817225 \\
\hline $\mathrm{H}$ & -3.1412670014 & 5.2197574796 & -3.2605839192 \\
\hline $\mathrm{H}$ & -4.1490397936 & 3.6605965864 & -4.9130718048 \\
\hline 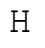 & -4.2801596806 & 1.2355497757 & -4.387161481 \\
\hline & -3.4131337954 & 0.381814568 & -2.2623621893 \\
\hline $\mathrm{H}$ & -4.5393755685 & 2.5862751183 & 0.8497466809 \\
\hline $\mathrm{H}$ & -5.3562751155 & 4.406814481 & 2.3118371309 \\
\hline $\mathrm{H}$ & -3.7444078225 & 5.9505367913 & 3.4001556255 \\
\hline $\mathrm{H}$ & -1.3129092059 & 5.6845742041 & 2.9908552619 \\
\hline $\mathrm{H}$ & -0.4915119855 & 3.8844269605 & 1.5179973967 \\
\hline 11 & 011131 & 09460113 & \\
\hline & 1.9061208843 & -3.9284496 & \\
\hline
\end{tabular}




\begin{tabular}{|c|c|c|c|}
\hline T & 1.0020755011 & -6.2219222055 & 1.9376936712 \\
\hline & -4.2296758599 & -2.6277056141 & -0.4659581209 \\
\hline & -5.6751834507 & -3.939154261 & -1.9648219574 \\
\hline & -4.8625297685 & -4.597523719 & -4.2175239669 \\
\hline & -2.5747268984 & -3.9560024173 & -4.9367275502 \\
\hline & -1.1163027989 & -2.6763436812 & 87542 \\
\hline & -0.9368171143 & 66728422 & .761 \\
\hline & -1.9646925161 & -4.6490571118 & -0.7199582158 \\
\hline & 8.7252787631 & 0.3055148456 & 2.9520624257 \\
\hline & 094876 & -0.971345978 & 1.7315505489 \\
\hline & 18825 & 73708105 & 3.4751300901 \\
\hline & 9.4218969058 & 2.376425 & -1.120 \\
\hline & 8.1042008246 & 2.54 & 0.08 \\
\hline & 7.9941268507 & 3.4001263374 & -1.4790261739 \\
\hline & -3.2130883544 & 1.640980759 & 3.2792444025 \\
\hline & -4.9706988791 & 0.001204532 & 2.7357288496 \\
\hline & -2.0000805338 & -3.0824199523 & 2.295 \\
\hline & -0.2128722434 & -1.41125 & 2.803 \\
\hline & -5.2318383833 & -2.9417522877 & 3.2193507746 \\
\hline & -4.3271612299 & -3.6143680133 & 1.850982235 \\
\hline & -5.4651868062 & -2.2692082718 & 1.5953526115 \\
\hline & -0.57736 & 422409 & 4.6 \\
\hline & 0.49 & 0.81 & 3.32 \\
\hline & -0.6577415531 & 2.1790297397 & 3.1737021494 \\
\hline & 5.3624657297 & -2.8993061031 & 2.0758977315 \\
\hline & 3.6 & -2.4 & 736213 \\
\hline & 4.9 & -2 . & 48 \\
\hline & 7.6 & -0.7 & -3 . \\
\hline & 6.16 & -1.566 & -3.509 \\
\hline & 6.4829922818 & 0.0709925389 & -4.1238981248 \\
\hline & & & \\
\hline & & & \\
\hline $\mathrm{C}$ & -3.9651857841 & 0.6763640959 & -1.9120533477 \\
\hline & -3.4 & -0.4104977207 & -2.6562963679 \\
\hline & -3.8487034782 & -1.7179152802 & -2.2130806252 \\
\hline & -4.7027669476 & -1.9044297815 & -1.1232820933 \\
\hline & -5.2834169199 & -0.785933585 & -0.4383122329 \\
\hline c & -4.8755422472 & 0.49629914 & -0.8277584067 \\
\hline$u$ & -2.9573663971 & -0.6637142223 & -0.373783986 \\
\hline $\mathrm{Cl}$ & -3.1832695366 & -1.8542436124 & 1.7033712668 \\
\hline C & -2.7273565723 & -0.2107660359 & -3.9402299052 \\
\hline & -6.277243793 & -1.0165980597 & 0.658824496 \\
\hline- & -2.0772708285 & 1.2349948358 & 0.8074917201 \\
\hline C & -2.8321705549 & 1.53473068 & 2.453064287 \\
\hline C & -4.1369744103 & 1.114 & 238159 \\
\hline C & -4.7651200942 & 1.450725151 & 3.9096905994 \\
\hline C & -4.0916392483 & 2.2098566311 & 4.8613144303 \\
\hline $\mathrm{C}$ & -2.7894834874 & 2.6348949324 & 4.6108226835 \\
\hline $\mathrm{C}$ & -2.1634290026 & 2.3 & 4061 \\
\hline $\mathrm{C}$ & -0.2867481598 & 1.3265008357 & 1.2130499382 \\
\hline $\mathrm{C}$ & 0.1802897078 & 0.4389858521 & 2.1874 \\
\hline$C$ & 1.5175468088 & 0.4313466055 & 2.5588301723 \\
\hline $\mathrm{C}$ & 2.3982679516 & 1.3446618015 & 1.9905277425 \\
\hline $\mathrm{C}$ & 1.9366523992 & 2.2669051801 & 1.0499041158 \\
\hline $\mathrm{C}$ & 54013 & 2.2409 & 79101 \\
\hline $\mathrm{C}$ & 2.8372581228 & 3.3022527292 & 0.4400449967 \\
\hline 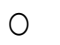 & 2.6212872645 & 3.7874543109 & -0.6724102314 \\
\hline
\end{tabular}




\begin{tabular}{|c|c|c|c|}
\hline $\mathrm{N}$ & 3.8915122211 & 3.6846245847 & 1.1830318214 \\
\hline & 4.8888923526 & 4.6159174379 & 0.6975623529 \\
\hline & 4.4012311728 & 6.0700927199 & 0.7076521554 \\
\hline & 6.1077951214 & 4.4391943936 & 1.5916928341 \\
\hline & 6.1077771799 & 3.771287903 & 2.6026176129 \\
\hline 0 & 7.1477017746 & 5.1255682348 & 1.136873023 \\
\hline & 8.3359481903 & 5.0661350607 & 1.9378243023 \\
\hline & -2.4128089099 & 2.8305044817 & -0.0496892139 \\
\hline & -1.93635 & 092 & 32749 \\
\hline & -2.1813112039 & 4.1994712067 & -2.0443921503 \\
\hline & -2.911493443 & 5.2190275997 & -1.4373324326 \\
\hline & -3.3923855268 & 5.0435004961 & -0.1436432243 \\
\hline & -3.1480916148 & 3.858337595 & 0.5496088895 \\
\hline & -0.83056117 & -1.792150159 & -0.6474804775 \\
\hline & -1.0349731806 & -3.0371813939 & 13002 \\
\hline & -1.7948512138 & -4.1762110295 & -1.681293724 \\
\hline C & -2.0330885387 & -5.1372429594 & -2.6547821732 \\
\hline & -1.5133964356 & -4.9729256173 & -3.9406044022 \\
\hline & -0.743 & -3.8548135322 & 678899 \\
\hline & -0.4977807008 & -2.8885323749 & -3.2608050701 \\
\hline & 0.5227828203 & -0.7116023784 & -1.2520649026 \\
\hline C & 0.1697696058 & 0.3094589672 & -2.1286319161 \\
\hline C & 1.1052637566 & 1.2242650129 & -2.59410459 \\
\hline & 2.4465148341 & 1.0726973751 & -2.2417772637 \\
\hline C & 2.816 & 0.036975 & $-1 \cdot 35$ \\
\hline $\mathrm{C}$ & 1.86 & -0.8375 & -0 \\
\hline C & 0.606010 & 2.3821298836 & -3.4091084503 \\
\hline & -0.3475672842 & 2.2686522309 & -4.1809639484 \\
\hline $\mathrm{N}$ & 1.2408410554 & 3.5421563324 & -3.1525820098 \\
\hline C & 1.0 & 4.7 & -3.94 \\
\hline C & 0.9 & 5.9 & 2129 \\
\hline $\mathrm{C}$ & 2.2732871 & 4.8650581905 & -4.8925165428 \\
\hline 0 & 3.2650472589 & 4.1786857316 & -4.8509405132 \\
\hline 0 & 2.0791186276 & 5.8625555125 & -5.7597480574 \\
\hline & 3.1 & 6.0 & -6.683321 \\
\hline C & 0.0 & -2.8672229902 & 8093 \\
\hline $\mathrm{C}$ & 0.975981192 & -3.771871032 & 0.0732362267 \\
\hline $\mathrm{C}$ & 1.7412429824 & -4.562271552 & 0.9210900505 \\
\hline $\mathrm{C}$ & 1.5714652365 & -4.4742471545 & 2.3005827396 \\
\hline $\mathrm{C}$ & 0.627 & -3.5966508217 & 2.8185816179 \\
\hline$C$ & -0.13 & 34684 & 67751 \\
\hline $\mathrm{H}$ & 5.161 & 4.3404427802 & -0.3266392643 \\
\hline $\mathrm{H}$ & 0.1809533441 & 4.5996265371 & -4.5647214531 \\
\hline $\mathrm{H}$ & 1.8962429181 & 3.5798253801 & -2.3752351963 \\
\hline $\mathrm{H}$ & 4.0 & 3.3335927464 & 2.1186709174 \\
\hline $\mathrm{H}$ & 3.4466650478 & 1.3171446237 & 2.2722450978 \\
\hline$H$ & 1.8693329101 & -0.2849886945 & 3.2932623331 \\
\hline $\mathrm{H}$ & -0.5150875094 & -0.2312695555 & 2.678280409 \\
\hline $\mathrm{H}$ & 0.2794469406 & 2.9578880696 & -0.1016985067 \\
\hline $\mathrm{H}$ & -0.8606462109 & 0.424019901 & -2.433581682 \\
\hline $\mathrm{H}$ & 2.1708828268 & -1.6047531701 & -0.1768483145 \\
\hline$H$ & 3.856755342 & -0.0818645746 & -1.1064011111 \\
\hline $\mathrm{H}$ & 3.1894189283 & 1.7634529799 & -2.627186307 \\
\hline $\mathrm{H}$ & 1.123040036 & -3.8608379831 & -0.9980491065 \\
\hline $\mathrm{H}$ & 2.4675078094 & -5.2494028262 & 0.4997017658 \\
\hline $\mathrm{H}$ & 34999109 & -5.095530432 & 2.9647226349 \\
\hline 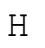 & 0.4676970477 & -3.5358620471 & 3.8902386824 \\
\hline
\end{tabular}




\begin{tabular}{|c|c|c|c|}
\hline 4 & & & \\
\hline & -2.1988540047 & $-4 \cdot 30$ & \\
\hline & -2.6203763672 & -6.0171187641 & -2.4131844687 \\
\hline & -1.7022385975 & -5.7226211417 & -4.7017064568 \\
\hline & -0.324402643 & -3.7301578706 & -5.2316448266 \\
\hline & 0.1166051541 & -2.0263749382 & -3.5010584407 \\
\hline & -1.3872 & 2.2352955505 & -1.8 \\
\hline & -1.8074485139 & 4.2951381809 & -3.0587882552 \\
\hline & -3.1088212568 & 6.1427526845 & -1.9714749691 \\
\hline & -4.6586020056 & 0.5001581879 & 1.9961184043 \\
\hline & -5.7769642965 & 1.1081993652 & 4.09 \\
\hline & -4.576881552 & 2.4658114993 & 5.797 \\
\hline & -2.2579406639 & 3.2288825461 & 5.3468200261 \\
\hline & -1.1540468342 & 2.6561186465 & 3.2285929646 \\
\hline & -3.9627681119 & 5.8319445408 & 0.3362573057 \\
\hline & -3.5 & 3.7459572875 & 1081 \\
\hline 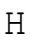 & 3.29974 & 5.21 & 849252 \\
\hline  & 4.07375 & 6.313 & -6.1486383562 \\
\hline & 2.8389229663 & 6.9476230609 & -7.2840765619 \\
\hline & 618337 & 5.6670861547 & 1.4094037405 \\
\hline $\mathrm{H}$ & 090924 & 38549099 & 26217 \\
\hline $\mathrm{H}$ & 323472 & 5.4774074215 & 2.9302584907 \\
\hline & -3.4444567961 & -2.581647828 & -2.7255210612 \\
\hline & -4.9297327433 & -2.9079593667 & -0.7810487593 \\
\hline & 5738125 & 1.3681643344 & -0.3019824329 \\
\hline $\mathrm{H}$ & -3.6845836842 & 1.6812536792 & -2.1986852809 \\
\hline & -7.1 & -1.58673487 & 0.26 \\
\hline H & 7522723 & 353614 & 68456 \\
\hline $\mathrm{H}$ & -5.8185626807 & -1.5971488732 & 1.4654456412 \\
\hline & -3.4536705909 & -0.2316363058 & -4.760302996 \\
\hline & -2.1987212196 & 0.7449962802 & 33043 \\
\hline $\mathrm{H}$ & -2.0146073202 & -1.0179617948 & 14375 \\
\hline $\mathrm{H}$ & 0.8 & 6.86 & -3.6 \\
\hline $\mathrm{H}$ & 543837 & 5.86 & -2.4 \\
\hline $\mathrm{H}$ & 1.8242539896 & 6.0880182207 & -2.4318409281 \\
\hline $\mathrm{H}$ & $5.1 \varepsilon$ & 6.731 & 0.331 \\
\hline $\mathrm{H}$ & 3.5 & 6.154188849 & 0.066 \\
\hline & 4.1321757494 & 6.3781302894 & 1.7217717956 \\
\hline & & & \\
\hline & & & \\
\hline $\mathrm{C}$ & -7.2729581357 & 1.77 & 2549583 \\
\hline 0 & -7.0668308476 & 2.1953559251 & 2.3305847341 \\
\hline C & -6.737180014 & -2.5352105457 & -4.0183261747 \\
\hline O & 151254155 & -2.9214236532 & -2.6574950423 \\
\hline C & -6.0004634738 & -1.997541396 & -1.8604384455 \\
\hline C & -5.8732112223 & -2.5139481091 & -0.4303210786 \\
\hline $\mathrm{C}$ & -6.371550461 & 1.3721195863 & 1.5616433561 \\
\hline C & -6.2556326777 & 1.9130492083 & 0.1387045348 \\
\hline O & -5.9334997457 & 0.3051971599 & 1.9524502729 \\
\hline 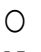 & -5.7244799427 & -0.8729627523 & -2.2372016058 \\
\hline N & -5.093907783 & 1.3838178647 & -0.5295572271 \\
\hline $\mathrm{C}$ & -3.8712448353 & 1.8072050824 & -0.1245986894 \\
\hline $\mathrm{N}$ & -4.8371060176 & -1.8214186951 & 0.2933777583 \\
\hline $\mathrm{C}$ & -3.5438181732 & -2.0787490427 & -0.0211375707 \\
\hline O & -3.7423493653 & 2.5913832745 & 0.8100711285 \\
\hline 0 & -3.2 & -2.8267569057 & -0.9502409127 \\
\hline & -7.21417694 & -2 & 0.29711061 \\
\hline
\end{tabular}




\begin{tabular}{|c|c|c|c|}
\hline & -5.5976869585 & -3.5686268611 & 22221 \\
\hline & -6.138680652 & 2.9954958491 & 0.2377027426 \\
\hline & -7.5258461581 & 1.6030038657 & -0.656207438 \\
\hline & -5.2155376761 & 0.5826632 & .1458913533 \\
\hline & -5.1103965788 & -1.0708642069 & 0.9238368223 \\
\hline & -2.471134774 & -1.4483996514 & 819 \\
\hline & -2.698581866 & -0.6276395652 & 1271 \\
\hline & -3.7030321873 & -0.3669218273 & 2.2488723144 \\
\hline & -1.6138328199 & -0.1238456468 & 2.645903738 \\
\hline & -1.7842365717 & 0.5120545574 & 3.5076602151 \\
\hline & -0.3141117211 & -0.4309866792 & 2.2650715884 \\
\hline & 0.5181166377 & -0.0726832047 & 2.8596395562 \\
\hline & -0.0769227977 & -1.231228003 & 1.1415606127 \\
\hline & 1.6623056036 & -1.594605311 & 0.689568308 \\
\hline & -1.1635446868 & -1.7446787207 & 0.443229365 \\
\hline & -1.0517293299 & -2.4029689812 & -0.4070843675 \\
\hline & -2.6690023601 & 262017 & -0.87 \\
\hline & -1.4338181892 & 1.6750577179 & -0.3471320972 \\
\hline & -1.4469010351 & 2.2754185394 & 0.5533184215 \\
\hline & -0.2463218003 & 1.2741996039 & -0.9485817601 \\
\hline & 1.3883108688 & 1.7913217025 & -0.2946182992 \\
\hline & -0.3109213424 & 0.471042532 & -2.09 \\
\hline & 0.6082364576 & 0.1379850075 & -2.557492517 \\
\hline & -1.5363948837 & 0.1023618526 & -2.6307648732 \\
\hline & -1.5735717864 & -0.5216865029 & -3.5176882162 \\
\hline & -2.7223305405 & 0.5228055692 & -2.0320394255 \\
\hline & -3.6683462173 & 0.2175406786 & -2.465874395 \\
\hline & 932931932 & 4.3983476491 & -0.3092 \\
\hline & 0.2981525369 & 4.1242804463 & 0.7349354664 \\
\hline & -0.1972387757 & 4.9868316149 & 1.7030813322 \\
\hline & -0.669462775 & 5.9152202844 & 1.4005980638 \\
\hline & -0.0942621104 & 4.655969908 & 3.0517678624 \\
\hline & -0.47 & 045 & 3.8 \\
\hline & 0.5062012243 & 3.4585745887 & 3.4188780753 \\
\hline & 0.6074103476 & 3.1963252426 & 4.4669634965 \\
\hline & 0.9987279095 & 2.5888340697 & 2.4488747021 \\
\hline & 1.4968639386 & 1.6839281943 & 2.7564215579 \\
\hline & 0. & 2.9 & 1.09 \\
\hline & 1.9616 & 3.0716968114 & -1.4859864913 \\
\hline & 3.033601747 & 3.8801301711 & -1.0802020697 \\
\hline & 3.4558147864 & 3.7565329407 & -0.085661981 \\
\hline & 3.5573061396 & 4.8347419913 & -1.9421515337 \\
\hline & 4.3833549502 & 5.4591417284 & -1.6174831716 \\
\hline & & 7192 & -3.2196543682 \\
\hline & 3.4257501552 & 5.7422497323 & -3.8931818419 \\
\hline & 1.941280224 & 4.2120798381 & -3.6182857961 \\
\hline & 1.5008993302 & 4.348585373 & -4.6006132262 \\
\hline & 1.4085428888 & 3.2555883554 & -2.7515535637 \\
\hline & 0.5514155532 & 2.6695210576 & -3.0668702246 \\
\hline & 1.5500753738 & -3.0173607895 & -0.4731564352 \\
\hline & 0.9006392315 & -2.8662081603 & -1.7065687884 \\
\hline & 0.4132934284 & -1.9290349889 & -1.9489067656 \\
\hline & 0.8753890425 & -3.9028352598 & -2.6302716109 \\
\hline & 0.3514709572 & -3.7721109897 & -3.5714988217 \\
\hline & & -5.1060424369 & -2.3470802522 \\
\hline & 1.5065170298 & -5.9150551552 & -3.0696093816 \\
\hline & 4.3145598877 & 5787816 & 1.9795849715 \\
\hline
\end{tabular}




\begin{tabular}{|c|c|c|c|}
\hline 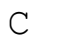 & 3.6360518231 & -2.3609042293 & 2.553306649 \\
\hline C & 4.1136797433 & -3.0755961956 & 3.6474531582 \\
\hline 4 & 5.1669649289 & -3.0242959569 & 3.9033093277 \\
\hline & 3.2406651659 & -3.8360476665 & 4.4183221832 \\
\hline & 3.6108949954 & -4.3879385062 & 0270218 \\
\hline & 1.8877466311 & -3.8820384633 & 4.0896096523 \\
\hline & 1.2019355924 & -4.474023455 & 4.68 \\
\hline & 1.409538651 & -3.1719076697 & 2.99 \\
\hline & 0.3548726595 & -3.2264318648 & 2.7444606569 \\
\hline $\mathrm{Ru}$ & 3.0959929685 & 0.0891402953 & -0.2268098007 \\
\hline & 2.282364 & -2.40 & 2.2155297168 \\
\hline & 2.17 & -5.263 & $-1 \cdot 1$ \\
\hline & 2.1874410288 & -4.2292595025 & -0.1959180302 \\
\hline & 2.6773189211 & -6.1976935725 & -0.9011262829 \\
\hline & 2.6946003728 & -4.3781808876 & 0.7510437962 \\
\hline $\mathrm{Cl}$ & 3.6227 & 0.8749 & 6199 \\
\hline & -6.3129328806 & 1.6545468304 & 4.18958 \\
\hline & -7.820706406 & 0.8341436303 & 3.714428 \\
\hline & -7.851191 & 2.5733842877 & 4.1511707002 \\
\hline & -7.1883320832 & -3.4014356094 & -4.4978502405 \\
\hline & -5.7 & -2.2 & -4.4 \\
\hline & -7.40 & -1.674 & -4.06 \\
\hline & 3.5 & -0.0669915121 & -2.5168012839 \\
\hline & 4.26 & 1.042 & -1.9642352671 \\
\hline & 5.1267591305 & 0.8857659561 & -0.878859464 \\
\hline$c$ & 5.3 & 097375335 & -0.3184076673 \\
\hline $\mathrm{C}$ & 4.6 & -1.2 & -0 . \\
\hline r & 3.7 & -1.31 & -1.8957761646 \\
\hline & 4.1088776478 & 2.0279034776 & -2.385542109 \\
\hline 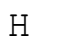 & 5.612353 & 1.7536580266 & -0.447055661 \\
\hline $\mathrm{C}$ & 621434 & -0.5686397612 & 0.7795241883 \\
\hline $\mathrm{H}$ & 4.77 & -2.4 & $-0.3-3+x-1$ \\
\hline $\mathrm{H}$ & 3.1 & -2.1 & 94711 \\
\hline $\mathrm{H}$ & 7.35 & -0.1857563347 & 0.443150605 \\
\hline 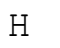 & 6.510 & -1.6164775418 & 9800659 \\
\hline $\mathrm{H}$ & 6.07 & 0.0060792112 & 627329 \\
\hline $\mathrm{C}$ & 2.77 & 15633 & -3.795 \\
\hline$H$ & 3.47 & 0.00 & -4.63 \\
\hline $\mathrm{H}$ & 2.051422 & -0.7412193382 & 2261538 \\
\hline $\mathrm{H}$ & 2.270949 & 1.0314408794 & -3.8622077741 \\
\hline & -7.1351964188 & -2.8448182184 & 1.2862647609 \\
\hline $\mathrm{H}$ & -7.49274 & -1.3409549571 & 35658 \\
\hline U & -8.0 & -2.8 & 187 \\
\hline $\mathrm{H}$ & -7.4 & 2.0 & 43976 \\
\hline 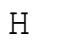 & -7.6541080373 & 0.5262467305 & -0.7942945661 \\
\hline & -8.4023467891 & 2.0019223674 & -0.1423791903 \\
\hline & & & \\
\hline & IIm & & \\
\hline $\mathrm{C}$ & -3.9007966063 & 0.7885238827 & -1.6773767786 \\
\hline  & -3.3201836282 & -0.1495824091 & -2.5468265787 \\
\hline$C$ & -3.6189459093 & -1.5245684959 & -2.3109303589 \\
\hline $\mathrm{C}$ & -4.463845597 & -1.9206603523 & -1.2696247713 \\
\hline $\mathrm{C}$ & -5.1140844844 & -0.9511599242 & -0.4380575913 \\
\hline $\mathrm{C}$ & -4.7995404444 & 0.398719664 & -0.6430812445 \\
\hline $\mathrm{Ru}$ & -2.791851436 & -0.6817047641 & -0.3380390666 \\
\hline $\mathrm{Cl}$ & 068 & -2.050070661 & 1.6275122886 \\
\hline 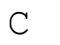 & -2.5 & .2 & \\
\hline
\end{tabular}




\begin{tabular}{|c|c|c|c|}
\hline & -6.0876676204 & -1.3907581537 & 0.611233331 \\
\hline & -1.9458251655 & 1.1409243073 & 0.9657289946 \\
\hline & -2.7897516614 & 1.4026457438 & 2.5723287306 \\
\hline & -4.0269594082 & 0.8185347742 & 2.8468807216 \\
\hline & -4.7014132122 & 1.1130211072 & 4.028214072 \\
\hline & -4.1443471388 & 1.995487519 & 4.9474366948 \\
\hline & -2.909601455 & 2.5842671696 & 4.6823147295 \\
\hline & -2.2345007622 & 2.2924314328 & 3.5024479118 \\
\hline & -0.181881127 & 1.290338704 & 1.4510461315 \\
\hline & 0.3272641894 & 0.422998549 & 2.4251578443 \\
\hline & 1.653567974 & 0.5213047665 & 2.8293590241 \\
\hline & 2.487477652 & 1.4951818577 & 2.2814660604 \\
\hline & 1.9772756659 & 2.3859860083 & 1.3401701846 \\
\hline & 0.646554478 & 2.2845368747 & 0.9412190968 \\
\hline & 2.7568837646 & 3.522317175 & 0.7343776327 \\
\hline & 2.1896040141 & 4.5512680579 & 0.3853104396 \\
\hline & 4.0929751199 & 3.3408882878 & 0.6077964754 \\
\hline & 4.8850478647 & 4.3918577998 & 0.0133353933 \\
\hline & 6.3679990192 & 4.0188332374 & 0.0673847888 \\
\hline & 4.479385506 & 4.651327 & 372086483 \\
\hline & 4.0686355287 & 3.8186058839 & -2.2150274524 \\
\hline & 4.7089353282 & 5363 & -1.766867 \\
\hline & 4.4300469768 & 6.2701987206 & -3.1238038725 \\
\hline & -2.2870128713 & 2.7187480618 & 0.0822939638 \\
\hline & -1.6324509233 & 2.9943907482 & -1.126942086 \\
\hline & -1.929076777 & 4.1415901301 & -1.8505924204 \\
\hline & -2.9053963172 & 5.0241500412 & -1.3888492514 \\
\hline & -3.5692594135 & 4.7536736035 & -0.1984429431 \\
\hline & -3.2593073264 & 3.610333913 & 0.5391029676 \\
\hline & -0.6586154977 & -1.7298748299 & -0.686467938 \\
\hline & -0.8191750281 & -2.8228662316 & -2.1554746998 \\
\hline & -1.5987579145 & -3.9771150091 & -1.9881741089 \\
\hline & -1.820837508 & -4.835191685 & -3.0565588679 \\
\hline & -1.2662907098 & -4.5528203907 & -4.306675351 \\
\hline & -0.476453895 & -3.4213958715 & -4.4723332185 \\
\hline & -0.2454423329 & -2.5596073 & -3.3975495096 \\
\hline & 0.7745328558 & -0.6443206048 & -1.0695854077 \\
\hline & 1.9794711876 & -0.8207407489 & -0.3940551239 \\
\hline & 3.0901152145 & -0.0323057972 & -0.6816372203 \\
\hline & 2.9989057981 & 0.983108095 & -1.6290737632 \\
\hline & 1.794913541 & 1.1777920672 & -2.3014241286 \\
\hline & 0.6946529274 & 0.3687778783 & -2.0303872556 \\
\hline & 4.3572 & -0.2564012012 & 0.0856654656 \\
\hline & 5.1117451506 & 0.6639033948 & 0.4116721379 \\
\hline & 4.6075868124 & -1.5365616488 & 0.4255307878 \\
\hline & 5.7372492206 & -1.9134631189 & 1.2469059747 \\
\hline & 5.5081180484 & -1.5768992947 & 2.7261736899 \\
\hline & 5.9398005785 & -3.4086648381 & 1.0547257423 \\
\hline & 5.1641841824 & -4.1260124692 & 0.4601543766 \\
\hline & 7.0555072445 & -3.8257641401 & 1.6386972533 \\
\hline & 7.327553366 & -5.2297787613 & 1.5384120364 \\
\hline & 0.1532845914 & -2.926183462 & 0.4663856784 \\
\hline & 1.0724333997 & -3.8283524093 & -0.0972749935 \\
\hline & 1.9063814344 & -4.5939630402 & 0.7079836794 \\
\hline & 1.8335877281 & -4.4802944857 & 620206 \\
\hline & 0.9105795908 & -3.6116711127 & 2.6637401454 \\
\hline & 0.078437 & -2.8386881968 & $1.856228 \varepsilon$ \\
\hline
\end{tabular}




\begin{tabular}{|c|c|c|c|}
\hline $\mathrm{H}$ & 4.7100055061 & 5.3222987146 & 0.5596372776 \\
\hline $\mathrm{H}$ & 6.6324810975 & -1.3902446279 & 0.8949657875 \\
\hline & 4.0636405305 & -2.3025247938 & 0.0480766526 \\
\hline & 4.4764507797 & 2.3941704185 & 0.6046933981 \\
\hline & 3.5210084882 & 1.5757601292 & 2.6025065387 \\
\hline & 2.0341163954 & -0.1565758276 & 3.5864035065 \\
\hline & -0.3251940577 & -0.3028944861 & 2.8965250311 \\
\hline & 0.2921783305 & 3.0325867453 & 0.245 \\
\hline & 2.0542228435 & -1.5544591605 & 0.3961460025 \\
\hline & -0.2354227259 & 0.5290905656 & -2.5647028169 \\
\hline & 1.7232949462 & 1.9760153476 & -3.0324742858 \\
\hline & 3.8 & 1.6471739802 & -1.821578433 \\
\hline & 1.1685391143 & -3.9164791083 & -1.1738475097 \\
\hline & 2.6299103772 & -5.260115249 & 0.2521147455 \\
\hline & 2.4887943371 & -5.0740785849 & 2.7238334205 \\
\hline & 0.8260962083 & -3.5299103157 & 3.7426062781 \\
\hline & -0.6472992839 & -2.187277287 & 2.3147004681 \\
\hline & -2.0330902049 & -4.1966 & -1.0155823801 \\
\hline & -2.420795 & -5.7283266118 & -2.9158652939 \\
\hline & -1.4405357555 & -5.2228363082 & -5.1419602858 \\
\hline & -0.0229782113 & -3.209990495 & -5.4351562837 \\
\hline & 0.3963521794 & -1.6953696636 & -3.5350091303 \\
\hline & -0.8 & 2.30 & -1.50 \\
\hline & 75277932 & 4.34 & -2.7736788398 \\
\hline & -3.1404912037 & 5.9206836585 & -1.952578553 \\
\hline & -4.4483484864 & 0.1016655456 & 2.156363062 \\
\hline & 6191568 & 0.6389441631 & 4.2334777523 \\
\hline & -4 & 2.22 & 5.87 \\
\hline & -2.4 & 3.27 & 5.35 \\
\hline & -1.2779029316 & 2.7657693831 & 3.3049066462 \\
\hline & -4.32725116 & 5.4373865159 & 0.1692418627 \\
\hline & -3.779219444 & 3.423422166 & 1.4724367175 \\
\hline & 7.42 & -5.5216 & 0.4910654791 \\
\hline & 6.5 & -5.80 & 2.001 \\
\hline & 8.2654074562 & -5.3838496049 & 2.0678267669 \\
\hline & 4.6649637322 & 7.3293493543 & -3.2127813207 \\
\hline & 3.3770000811 & 6.091410568 & -3.350787877 \\
\hline & 5.048238228 & 5.6811392056 & -3.8049997722 \\
\hline & -3.1633577963 & -2.2786546156 & -2.9394012889 \\
\hline & -4.6294220915 & -2.9756801834 & -1.0823989118 \\
\hline & -5.2325819648 & 1.1604355391 & -0.0039712796 \\
\hline & -3.6863141852 & 1.8398291078 & -1.8153365152 \\
\hline & -6.9227071968 & -1.9112429772 & 0.1329378693 \\
\hline & -6.4 & -0.5400110306 & 3233255 \\
\hline & 8795461 & -2.0794556877 & 3739453 \\
\hline & -3.2093576263 & 0.3506498896 & -4.6096877557 \\
\hline & -2.0883960015 & 1.2669932753 & -3.5988698 \\
\hline & -1.7557765939 & -0.4428717767 & -4.0005435343 \\
\hline $\mathrm{H}$ & 083187 & -1.875330163 & 3.325164497 \\
\hline $\mathrm{H}$ & 570333 & -0.4994569256 & 2.8255261843 \\
\hline & 4.6164169965 & -2.0913112848 & 3.0965678243 \\
\hline & 6.97469278 & 4.8094804311 & 0.3785574204 \\
\hline & 6.6788531918 & 3.8832285847 & 1.1057169794 \\
\hline & 6.547741525 & 3.0849934002 & -0.4729856079 \\
\hline & & & \\
\hline & & & \\
\hline
\end{tabular}




\begin{tabular}{|c|c|c|c|}
\hline C & L. 862217 & 1.420876 & 2.506338 \\
\hline C & 2.79804 & 0.560831 & 3.076857 \\
\hline & 2.896497 & 9591 & 9063 \\
\hline & 05 & 688 & \\
\hline & 513 & 22443 & 67 \\
\hline & .802636 & 1.646551 & 0.689729 \\
\hline & 3.260695 & 0.27308 & -0.651276 \\
\hline & 1.792324 & 0.802239 & 36285 \\
\hline & 0.001602 & 1.78593 & 0.343086 \\
\hline & -0.425267 & 2.520314 & -0.767755 \\
\hline & -1.772224 & 2.64089 & -1.056584 \\
\hline & -2.735032 & 2.052574 & -0.2 \\
\hline & -2.322008 & 847 & 0.8 \\
\hline & -0.9 & 1.187039 & 1.16 \\
\hline & -4.1 & 2.228692 & 0046 \\
\hline & -4.380535 & 2.814732 & 557 \\
\hline & -5.162265 & 1.716984 & 0.03 \\
\hline & -6.5 & 1.7 & -0 . \\
\hline & -6 . & 1.16 & -1.8 \\
\hline & -5.8 & 796 & 72848 \\
\hline & -7.356796 & 048 & 0011 \\
\hline & -7 & 1.20 & -4.039984 \\
\hline & 2.42 & 3.3 & 0.469246 \\
\hline & $2 . \varepsilon$ & 4.1 & 1. \\
\hline & 3.3 & 5.4 & 1. \\
\hline & 3.4 & 5.9 & 0. \\
\hline & 3.0 & 5.17 & -1 . \\
\hline & 2.5 & 3.89 & -0.82 \\
\hline & 7 & -1 & 057 \\
\hline C & 2.7 & -2 . & \\
\hline C & 2.6 & -2.6 & 12 \\
\hline 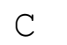 & 3. & -3.4 & 85 \\
\hline$c$ & 4. & -4.57 & 915 \\
\hline$c^{2}$ & & -4 & 21 \\
\hline 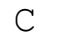 & 3 & -3. & \\
\hline C & 0.1 & -1.71 & -0.0 \\
\hline & -0 & 0508 & 35 \\
\hline & -2.085865 & -1.367539 & -0.80406 \\
\hline C & -2.5 & -1.934851 & 0.377207 \\
\hline 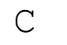 & -1.6 & -2 . & 87 \\
\hline C & -0.2 & 2374 & 9596 \\
\hline C & -4.0 & -2.111896 & 0.748571 \\
\hline D & $-4 \cdot 3$ & 231 & 1.791205 \\
\hline N & -4.9 & -1.615 & -0.06 \\
\hline$C$ & -6.36 & -1.760155 & 3441 \\
\hline C & -6.5 & -1.14 & 1.7 \\
\hline 0 & -5 & 7738 & 1015 \\
\hline o & -7. & -1.7938 & 2.504607 \\
\hline C & -7.591537 & -1.265147 & 3.819229 \\
\hline C & 1.91 & -2.740106 & -2.002178 \\
\hline C & 1.1 & -3.9 & -1 \\
\hline C & 1.1 & -4.7 & -3 \\
\hline$r$ & 1.7 & -4.410649 & -4.243768 \\
\hline$C$ & 2.5 & -3.22 & -4 . \\
\hline C & 2.57 & -2.392448 & -3.179503 \\
\hline 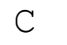 & 5.2 & -0.455913 & 952 \\
\hline & 5.05 & 0.932751 & .535364 \\
\hline
\end{tabular}




\begin{tabular}{|c|c|c|c|}
\hline & 4.979281 & 1.829978 & -0.568625 \\
\hline & 4.949977 & 1.350728 & -1.880447 \\
\hline & 4.974461 & -0.068374 & -2.07122 \\
\hline & 5.125828 & -0.945239 & -0.984789 \\
\hline & .640399 & -1.354397 & 1.479784 \\
\hline & .843185 & 2.268523 & -3.058864 \\
\hline & -6.939755 & -1.118146 & -0.31910 \\
\hline & 82275 & -3.19015 & 0.222412 \\
\hline & -7 & 3.207193 & - \\
\hline & -7.1 & 1.14128 & 0.1408 \\
\hline & -5.03 & 1.112381 & 0.844259 \\
\hline & 2328 & -0.998427 & -0.849806 \\
\hline & -2 . & -1.005433 & -1 \\
\hline & -0.3 & -0.8 & -1.93377 \\
\hline & 0.36 & -2.71 & 1.865045 \\
\hline & -2.06 & -2.840563 & 2.234409 \\
\hline & -2.108204 & 3.193254 & -1.926117 \\
\hline & 0.282657 & 3.000322 & -1.425632 \\
\hline & -0 . & 0.5 & \\
\hline & -3 & $0 . \varepsilon$ & 1.5 \\
\hline & 2.8 & 3.766 & 2.559579 \\
\hline & 3.7 & 6.006 & 2.183901 \\
\hline & 3.8 & 6.93 & -0.111551 \\
\hline & 3.1 & 5.5 & -2 \\
\hline & 2.2 & 3.2 & -1 \\
\hline & 0.6 & -4.2 & -1.06 \\
\hline & 0.5 & -5.67 & -3.03 \\
\hline & & -5.05 & -5.113653 \\
\hline & & -2.9 & -5 \\
\hline & 3.0 & -1. & -3 \\
\hline & -6 . & 1.2 & -4 . \\
\hline & -7 & 0.16 & -4.004302 \\
\hline & -8 & 1.8 & -4.57 \\
\hline & -8 & -1 & 4.282762 \\
\hline & -6 & -1.2 & 4.3 \\
\hline & -7 & -0.2 & 323 \\
\hline & 5.0 & 1.343179 & 1.53976 \\
\hline & 4.5 & 2.893 & -0.3831 \\
\hline & 4.8 & -0.4 & 77148 \\
\hline & & 2.0156 & -1.161269 \\
\hline & 5. & 2.1 & -3.6 \\
\hline & 3. & 2.008228 & -3.65604 \\
\hline & 4.7 & 3.3084 & -2.740765 \\
\hline & 6.7 & -1.312474 & 1.553684 \\
\hline $\mathrm{H}$ & & -2 & 4879 \\
\hline & & -1.0 & 2. \\
\hline & 2.1 & -1.7 & 2 . \\
\hline$H$ & 3.2 & -3.2 & 4.080045 \\
\hline $\mathrm{H}$ & & -5.23 & 3.286527 \\
\hline $\mathrm{H}$ & 4.6 & -5.687625 & 0.851498 \\
\hline & 3.5 & -4.2 & 67397 \\
\hline & 3.6 & -0.26 & 4.881719 \\
\hline & 2.1 & 1.045358 & 6.367067 \\
\hline & & 2.59857 & 5.376215 \\
\hline $\mathrm{H}$ & 0.29339 & 2.84567 & 2.934877 \\
\hline $\mathrm{H}$ & 3.442439 & -0.016353 & 2.428278 \\
\hline & -6.745529 & -3522680 & $-0.80 \xi$ \\
\hline
\end{tabular}




$\begin{array}{llll}\mathrm{H} & -6.338697 & -3.857099 & 0.891677 \\ \mathrm{H} & -7.944623 & -3.234716 & 0.468315 \\ \mathrm{H} & -7.062088 & 3.560485 & 0.606182 \\ \mathrm{H} & -6.530372 & 3.887021 & -1.060597 \\ \mathrm{H} & -8.138928 & 3.202066 & -0.759726\end{array}$

120

$2 \mathrm{pAaIIm}$

C $\quad-4.2871124993$

C $\quad-4.8518091908$

C $\quad-5.0302382381$

C $\quad-4.8049809585$

C $\quad-4.329406409$

C $\quad-4.0429252781$

$\mathrm{Ru} \quad-2.8807380444$

P $\quad-1.2282462412$

C $\quad-0.7639103533$

C $\quad-0.0443438705$

C $\quad 0.3755768845$

C 0.1039233989

C $\quad-0.5737424071$

C $\quad-1.0019359668$

C $\quad-5.3421106884$

C $\quad-4.0981190351$

P $\quad-2.2446919044$

C $\quad-3.096530201$

C $\quad-4.1298163571$

C $\quad-4.8561597165$

C $\quad-4.5577198663$

C $\quad-3.5356493679$

C $\quad-2.8200375374$

C $\quad-2.8188371602$

C $\quad-2.4940525392$

C $\quad-2.9519012952$

C $\quad-3.7352878937$

C $\quad-4.0541699617$

C $\quad-3.5967434369$

C $\quad-0.4811961303$

C $\quad 0.2022635851$

C $\quad 1.546490235$

C $\quad 2.2369456918$

C $\quad 1.5586218067$

C $\quad 0.2113740343$

C $\quad 3.6987669554$

N $\quad 4.4860071942$

C $\quad 5.925611606$

C $\quad 6.2600319444$

O $\quad 7.407361984$

C $\quad 7.783043365$

1. 9610538797

1.0333788038

$-0.2902939097$

$-0.6495304258$

0.3015269858

1.6149708938

0.1993653198

1.5644679379

3.0358975685

4.0726950021

5.1709738373

5.2409271946

4.1999236304

3.0991586891

1.4828351791

$-0.0621981088$

$-1.8539925649$

$-3.1768000282$

$-3.9277876405$

$-4.8345849581$

$-5.0095884009$

$-4.260070828$

$-3.3424706874$

$-2.1725921232$

$-3.380406275$

$-3.6382024288$

$-2.6965479745$

$-1.4954086181$

$-1.2415595362$

$-2.3210685352$

$-2.9285758528$

$-3.2427142004$

$-2.9488556665$

$-2.3544935996$

$-2.0406432277$

$-3.2937166419$

$-2.65065022$

$-2.7187444501$

$-2.2637357424$

$-2.7822106816$

$-2.4280889321$

$-0.3233792034$

$-4.0988278581$

$-1.4698944419$

2. 2828451577

1. 4825492714

1. 9628079294

3.2536356548

4.055136692

3.5746156533
$-0.9485746848$

$-0.0271133412$

$-0.4910263579$

$-1.8480874676$

$-2.7670370138$

$-2.2917581138$

$-0.9485183316$

0.0869975453

$-0.9163936231$

$-0.3033245819$

$-1.0413396836$

$-2.4072197443$

$-3.0297955681$

$-2.2894638865$

1.3208585505

$-4.2011452998$

0.0826874715

$-0.8809768814$

$-0.3101648454$

$-1.0810474289$

$-2.4271234437$

$-3.0068514374$

$-2.2466863712$

1. 7927942407

2.4300496671

3. 7154019171

4. 3851015168

3.7639352034

2. 4726172928

0.2071220001

$-0.8486798276$

$-0.7201623874$

0.4536786668

1.516593182

1. 3913043685

0.4630624342

1.3656873135

1.1903521368

$-0.2368154257$

$-0.6725996633$

$-2.0036887318$ $-2.6023898647$

$-0.3422794573$

$-0.8691681008$

1.6255103784

2. 7711134608

3. 9368261249

3. 9738153288

2.8377742149

1.6708805479 


\begin{tabular}{|c|c|c|c|}
\hline 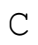 & 0.4769473212 & 1.0372734319 & 0.55230224 \\
\hline & 1.3255779707 & 0.5593207557 & -0.4559096306 \\
\hline & 2.6898069864 & 0.4165299796 & -0.2235358334 \\
\hline & 3.2193795625 & 0.7355867772 & 1.0270804578 \\
\hline & 2.3689046313 & 1.1367459961 & 2.0548038546 \\
\hline & 1.0124989924 & 1.3121164064 & 1.8143641069 \\
\hline & 4.6901928008 & 0.7153048994 & 1.3336705314 \\
\hline & 5.4438760492 & 1.4950424215 & 0.538919354 \\
\hline & 6.8339733465 & 1.7918136238 & 0.8147550964 \\
\hline & 7.146250302 & 3.0936864088 & 0.0883489727 \\
\hline & 8.3712267205 & 3.5253582807 & 0.369398686 \\
\hline & 8.7737540955 & 4.7284387571 & -0.2961269277 \\
\hline & 5.131569347 & 0.0955221514 & 2.3017746992 \\
\hline & 6.3768895841 & 3.653327994 & -0.6609253648 \\
\hline & 6.9513970826 & 1.9640845806 & 1.8910911484 \\
\hline & 7.7972656121 & 0.6796901247 & 0.3888312441 \\
\hline & 6.5175864 & .0694325541 & L. 5853023904 \\
\hline & 6.3336310457 & -1.9411856591 & 1.8453326013 \\
\hline & 4.1568334277 & -1.808737198 & 1.8200790089 \\
\hline & 5.0134287597 & 2.0534134821 & -0.1871955635 \\
\hline & 3.3457987125 & 0.0440612839 & -1.0048648315 \\
\hline & 28696865 & 0.3170168129 & 21647135 \\
\hline & 933 & 1.6974413549 & 2677412 \\
\hline & 2.7821197749 & 1.3531392454 & 3.0345835024 \\
\hline & 2.0873600669 & -3.7221049698 & -1.527930717 \\
\hline & -0.3002808667 & -3.166605614 & -1.7757835972 \\
\hline & -0.2978262707 & -1.5837834543 & 2.2331669379 \\
\hline & 2.0652063424 & -2.1416046652 & 2.4524274838 \\
\hline & -4.3835478148 & -3.8110058817 & 0.7370693815 \\
\hline & -5.6554941126 & -5.4057277009 & -0.6203017248 \\
\hline & -5.1192754389 & -5.7204886376 & -3.0240343638 \\
\hline & -3.2983930659 & -4.3831165566 & -4.0586844678 \\
\hline & -2.0774385917 & -2.7191055266 & -2.7317779921 \\
\hline & 0.195565696 & 4.0193613844 & 0.7544252207 \\
\hline & 0.9261902954 & 5.9678917594 & -0.5526373429 \\
\hline & 0.4381903331 & 6.0968998695 & -2.9839946205 \\
\hline & -0.7565251278 & 4.231570268 & -4.0989646455 \\
\hline & -1.469448082 & 2.2626968735 & -2.7925106915 \\
\hline & 7. & -2.7624749264 & 763416 \\
\hline & 7.9067232481 & -1.347083308 & -2.0989594393 \\
\hline & 8.7277233087 & -2.936439035 & -2.1890923118 \\
\hline & 9.7959246587 & 4.9151206088 & 0.0269993208 \\
\hline & 90967 & 4.5950520138 & -1.3786760616 \\
\hline & 5914117 & 5.5567874971 & -0.0097326434 \\
\hline & -5.3874261607 & -1.0484323811 & 0.1992519 \\
\hline & -4.9641467236 & -1.6734481762 & -2.1643078939 \\
\hline & -3.6339911209 & 2.3529722015 & -2.9697374129 \\
\hline & 65062298 & 2.9659616387 & -0.6085752855 \\
\hline & -4.9884996312 & 0.2043524965 & -4.7805415669 \\
\hline & -3.2393764339 & 0.4716461445 & -4.6102214547 \\
\hline & -3.9200509694 & -1.1333671284 & -4.3080369973 \\
\hline & -6.1543252474 & 2.2016060868 & 1.1746605083 \\
\hline & -4.5630969746 & 1.9772575019 & 1.9020121953 \\
\hline & -5.7431134302 & 0.6472390524 & 1.8966894453 \\
\hline & -1.6091622231 & 0.4732693683 & 2.7583232961 \\
\hline & -2.6388272177 & 1.3253887144 & 4.8139689584 \\
\hline & -3.5721874779 & 3.6328682808 & 4.8824485647 \\
\hline
\end{tabular}




\begin{tabular}{|c|c|c|c|c|c|}
\hline $\mathrm{H}$ & \multicolumn{2}{|c|}{-3.4561998292} & \multicolumn{2}{|c|}{5.061646559} & 2.8575786607 \\
\hline  & \multicolumn{2}{|c|}{-2.4057018418} & \multirow{2}{*}{\multicolumn{2}{|c|}{$\begin{array}{l}4.2206720309 \\
-0.7497325704\end{array}$}} & 0.8008477862 \\
\hline 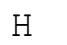 & \multirow{2}{*}{\multicolumn{2}{|c|}{$\begin{array}{l}-4.6515274686 \\
-4.0885307754\end{array}$}} & & & 4.2797011674 \\
\hline $\mathrm{H}$ & & & & 5.3902652569 \\
\hline $\mathrm{H}$ & \multicolumn{2}{|c|}{-2.6972457488} & \multicolumn{2}{|c|}{-4.5755261041} & 4.1984331101 \\
\hline $\mathrm{H}$ & \multirow{2}{*}{\multicolumn{2}{|c|}{$\begin{array}{l}-1.882775238 \\
-3.8157471103\end{array}$}} & \multicolumn{2}{|c|}{-4.1186926547} & 1.91982332 \\
\hline & & & \multirow{2}{*}{\multicolumn{2}{|c|}{$\begin{array}{r}-0.2959014353 \\
-4.0448123742\end{array}$}} & 1.9996374677 \\
\hline $\mathrm{H}$ & \multicolumn{2}{|c|}{7.6055153168} & & & 1.5055024822 \\
\hline $\mathrm{H}$ & \multicolumn{2}{|c|}{6.245368386} & \multicolumn{2}{|c|}{-4.2890305517} & 2.6201980193 \\
\hline $\mathrm{H}$ & \multicolumn{2}{|c|}{6.1332336035} & \multicolumn{2}{|c|}{-4.8580815945} & 0.9383911317 \\
\hline $\mathrm{H}$ & \multirow{2}{*}{\multicolumn{2}{|c|}{$\begin{array}{l}8.8293323216 \\
7.5945948225\end{array}$}} & \multicolumn{2}{|c|}{0.9869445375} & 0.566442325 \\
\hline 11 & & & -0.2163 & 3921779 & 0.9751300047 \\
\hline $\mathrm{H}$ & 7.6601302 & 254 & 0.44757 & 798038 & -0.6693891419 \\
\hline & 20 & & & & \\
\hline$n$ & Ip & & & & \\
\hline $\mathrm{C}$ & 1.50746 & -1.57 & 578822 & 2.57956 & \\
\hline C & 2.011189 & -0.3 & 366048 & 3.0765 & \\
\hline C & 3.403603 & -0.1 & .100839 & 2.8913 & \\
\hline $\mathrm{C}$ & 4.241395 & -1.0 & .05145 & 2.30552 & \\
\hline $\mathrm{C}$ & 3.748188 & -2.3 & .328175 & 1.8777 & \\
\hline $\mathrm{C}$ & 2.370747 & -2.5 & .555684 & 1.9986 & \\
\hline $\mathrm{Ru}$ & 2.609264 & & .636602 & 0.803 & 853 \\
\hline $\mathrm{Cl}$ & 4.514844 & & 0.474406 & -0.67 & 4515 \\
\hline $\mathrm{C}$ & 1.151073 & 0.55 & 555748 & 3.88878 & \\
\hline $\mathrm{C}$ & 4.69571 & -3.35 & 352243 & 1.33037 & \\
\hline $\mathrm{P}$ & 1.141863 & -1.5 & .583207 & -0.882 & 527 \\
\hline $\mathrm{C}$ & 1.951922 & -2.5 & .563249 & -2.203 & \\
\hline $\mathrm{C}$ & 3.19376 & -3.15 & 157459 & -1.9768 & \\
\hline $\mathrm{C}$ & 3.740901 & -4.0 & .031513 & -2.912 & 636 \\
\hline $\mathrm{C}$ & 3.051401 & -4.3 & 318274 & -4.085 & \\
\hline $\mathrm{C}$ & 1.807644 & -3.7 & .734128 & -4.318 & \\
\hline $\mathrm{C}$ & 1.256889 & -2.8 & .866952 & -3.382 & 553 \\
\hline $\mathrm{C}$ & 0.045969 & -0.4 & .472818 & -1.863 & 052 \\
\hline $\mathrm{C}$ & 0.676358 & 0.21 & 210424 & -2.9062 & \\
\hline $\mathrm{C}$ & 0.002099 & 1.16 & 169735 & -3.6479 & \\
\hline $\mathrm{C}$ & -1.324746 & & .443901 & -3.361 & 685 \\
\hline $\mathrm{C}$ & -1.994356 & & .732127 & -2.363 & 309 \\
\hline C & -1.311627 & & 0.23163 & -1.615 & \\
\hline $\mathrm{C}$ & -3.442342 & & .110895 & -2.197 & 091 \\
\hline 0 & -3.857911 & & .166314 & -2.666 & 692 \\
\hline $\mathrm{N}$ & -4.302814 & & .248422 & -1.596 & 879 \\
\hline $\mathrm{C}$ & -5.713217 & & .548007 & -1.717 & 028 \\
\hline $\mathrm{C}$ & -6.587256 & & 0.631297 & -1.28 & 9789 \\
\hline $\mathrm{C}$ & -6.084398 & & .783634 & -0.905 & 864 \\
\hline 0 & -5.596695 & & .08574 & 0.1696 & \\
\hline 0 & -7.065837 & & .459655 & -1.475 & 171 \\
\hline $\mathrm{C}$ & -7.525986 & & .624308 & -0.778 & 968 \\
\hline C & 0.03715 & -2.86 & 860508 & -0.1402 & \\
\hline $\mathrm{C}$ & -0.903413 & & 2.498415 & 0.827 & 291 \\
\hline $\mathrm{C}$ & -1.623651 & & 3.465398 & 1.520 & 047 \\
\hline $\mathrm{C}$ & -1.428934 & & 4.814484 & 1.242 & 348 \\
\hline $\mathrm{C}$ & -0.519577 & & 5.18764 & 0.2565 & \\
\hline $\mathrm{C}$ & 0.211434 & -4.2 & .220915 & -0.429 & 194 \\
\hline $\mathrm{P}$ & 2.144598 & 1.64 & 647332 & 0.19967 & \\
\hline $\mathrm{C}$ & 2.858141 & 2.74 & 746947 & 1.48632 & \\
\hline $\mathrm{C}$ & 4.255379 & $2.7 \varepsilon$ & 780921 & 1.59502 & \\
\hline $\mathrm{C}$ & 4.861693 & 3.59 & 593051 & 2.54476 & \\
\hline
\end{tabular}




\begin{tabular}{|c|c|c|c|}
\hline & .081735 & 4.382337 & 88 \\
\hline & 2.696841 & 4.362678 & 3.277225 \\
\hline & .083362 & 986 & 238 \\
\hline & .354145 & 2.063269 & 0.233811 \\
\hline & -0.451097 & 1.287521 & 1.059824 \\
\hline & -1.81267 & 1.532114 & 1.203914 \\
\hline & -2.389056 & 2.601087 & 35 \\
\hline & -1.59 & 3.3 & -0 . \\
\hline & -0.24 & 3.1 & -0.4 \\
\hline & -2.569691 & 0.530506 & 2.023137 \\
\hline & -1.972698 & -0.265883 & 2. \\
\hline & -3.9088 & 0.497264 & 1.874922 \\
\hline & -4.667936 & -0.574019 & 2.467735 \\
\hline & -4.184757 & -0.854877 & 162 \\
\hline & -4.61 & -1.811129 & 682 \\
\hline & -4.17 & -1.846455 & 0.4 \\
\hline & -5.1 & -2 . & 2.1 \\
\hline & -5.23 & -4.070143 & 1.4 \\
\hline & 2.795 & 2.518788 & -1.290 \\
\hline & 2.780 & 3.923062 & -1.27 \\
\hline & 3.193 & 4.653974 & -2.379 \\
\hline & 3.652 & 3.996496 & -3.518 \\
\hline & 3.7 & 2.6 & -3.5 \\
\hline & 3.286 & 1.87 & -2.4 \\
\hline & -5.923563 & 0.79561 & -2.76132 \\
\hline & -6.1 & -0.143294 & 2.7 \\
\hline & -4.37 & 1.059209 & 1.16 \\
\hline & -4.0 & -0 . & -1 \\
\hline & -1.87 & 2.2 & -3. \\
\hline & 0.519 & 1.70 & -4.43 \\
\hline & 1.7 & -0.0 & -3.15 \\
\hline & -1.829788 & -0.777564 & -0 \\
\hline $\mathrm{H}$ & -0 . & 0.4 & 1.5 \\
\hline & 0.34 & $3.7 C$ & -1.17 \\
\hline & -2.0 & 4.1 & -0.8 \\
\hline & -3.447856 & 2.821973 & 0.59 \\
\hline & 2.45 & 4.45 & -0.3 \\
\hline $\mathrm{H}$ & 3.1 & 5.7 & -2.3 \\
\hline & 3.9 & 4.5 & -4.3 \\
\hline & 4.0 & 2.08 & -4.3 \\
\hline & 3.39 & 0.80 & -2.432975 \\
\hline $\mathrm{H}$ & 4.8 & 2.16 & 0.933533 \\
\hline $\mathrm{H}$ & & & 2.6 \\
\hline $\mathrm{H}$ & & & 4. \\
\hline $\mathrm{H}$ & 2.0 & 4.9 & 3. \\
\hline $\mathrm{H}$ & 1.0 & 3.55 & 2.23 \\
\hline & -1. & -1.461535 & 775 \\
\hline $\mathrm{H}$ & -2.318959 & -3.149 & 2.290757 \\
\hline $\mathrm{H}$ & -1.97 & -5.571982 & 1.792751 \\
\hline $\mathrm{H}$ & 3.7 & -2.9 & -1 \\
\hline $\mathrm{H}$ & 4.7 & -4.4 & -2.7 \\
\hline $\mathrm{H}$ & 3.478 & -4.995673 & -4.817837 \\
\hline $\mathrm{H}$ & 1.262 & -3.957185 & -5.229703 \\
\hline $\mathrm{H}$ & 0.280097 & -2.43352 & -3.56915 \\
\hline $\mathrm{H}$ & -0.365189 & -6.2365 & 0.025413 \\
\hline & 0.928298 & -4.536606 & -1.178037 \\
\hline & -5.9493 & & \\
\hline
\end{tabular}




$\begin{array}{llll}\mathrm{H} & -4.257744 & -4.32887 & 0.980202 \\ \mathrm{H} & -5.575969 & -4.838131 & 2.091189 \\ \mathrm{H} & -8.357352 & 4.006684 & -1.36728 \\ \mathrm{H} & -7.852798 & 3.363191 & 0.229408 \\ \mathrm{H} & -6.725807 & 4.364271 & -0.720777 \\ \mathrm{H} & 3.810711 & 0.853236 & 3.203028 \\ \mathrm{H} & 5.283376 & -0.809065 & 2.129233 \\ \mathrm{H} & 1.944015 & -3.483155 & 1.632408 \\ \mathrm{H} & 0.451147 & -1.788016 & 2.686069 \\ \mathrm{H} & 5.319887 & -3.738915 & 2.142065 \\ \mathrm{H} & 4.161564 & -4.191586 & 0.880695 \\ \mathrm{H} & 5.353878 & -2.900267 & 0.582413 \\ \mathrm{H} & 1.379041 & 0.373174 & 4.944911 \\ \mathrm{H} & 0.087633 & 0.363901 & 3.731064 \\ \mathrm{H} & 1.374704 & 1.603083 & 3.682495 \\ \mathrm{H} & -7.63588 & -0.405233 & -1.493115 \\ \mathrm{H} & -6.474441 & -0.843984 & -0.225447 \\ \mathrm{H} & -6.306975 & -1.525806 & -1.850391 \\ \mathrm{H} & -6.686098 & -0.976167 & 3.149336 \\ \mathrm{H} & -6.596371 & 0.21414 & 1.828872 \\ \mathrm{H} & -6.113244 & 0.67521 & 3.463391\end{array}$

2mAs I Ip

$\begin{array}{llcc}\text { C } & 1.8531820075 & -3.8792302345 & 2.0680508037 \\ \mathrm{C} & 2.2975894655 & -2.7606417071 & 1.3454216409 \\ \mathrm{C} & 3.5437860275 & -2.2193448968 & 1.6386834523 \\ \mathrm{C} & 4.3495566197 & -2.7762234606 & 2.630177305 \\ \mathrm{C} & 3.9100900962 & -3.8904938742 & 3.3315043072 \\ \mathrm{C} & 2.6592931604 & -4.4430981507 & 3.0471441601 \\ \mathrm{P} & 1.1757723613 & -1.9411399653 & 0.1497597948 \\ \mathrm{Ru} & 2.1053965105 & -0.2283454389 & -1.2154317833 \\ \mathrm{Cl} & -0.1834612682 & 0.2618927449 & -1.8061247019 \\ \mathrm{C} & -0.2505703542 & -1.6471761839 & 1.2652841011 \\ \mathrm{C} & -1.5923253637 & -1.8023381681 & 0.9172944566 \\ \mathrm{C} & -2.5890445848 & -1.5966521177 & 1.8718818779 \\ \mathrm{C} & -2.2616112917 & -1.2497734746 & 3.1766181571 \\ \mathrm{C} & -0.921904141 & -1.1055506829 & 3.5357566548 \\ \mathrm{C} & 0.0658056721 & -1.2796202861 & 2.5747745844 \\ \mathrm{H} & -3.6292612334 & -1.7196573533 & 1.5899707191 \\ \mathrm{C} & 0.7004323179 & -3.3020935788 & -0.9952098961 \\ \mathrm{C} & 1.4583007657 & -4.4775338802 & -1.0597570908 \\ \mathrm{C} & 1.1961351164 & -5.4369800143 & -2.0362378388 \\ \mathrm{C} & 0.1728560872 & -5.2397608994 & -2.9572063553 \\ \mathrm{C} & -0.5841882633 & -4.0716558583 & -2.9027035008 \\ \mathrm{C} & -0.3182024958 & -3.1058077443 & -1.938599459 \\ \mathrm{P} & 1.9902427691 & 1.5758953443 & 0.352344598 \\ \mathrm{C} & 3.5559195324 & 1.6925965483 & 1.3021109715 \\ \mathrm{C} & 3.741202302 & 0.8811518461 & 2.4277017777 \\ \mathrm{C} & 4.9439674651 & 0.891733369 & 3.1255130842 \\ \mathrm{C} & 5.9860236403 & 1.7139371212 & 2.7039635119 \\ \mathrm{C} & 5.8133798611 & 2.5312737222 & 1.5900100374 \\ \mathrm{C} & 4.6069153801 & 2.5222951704 & 0.8937286337 \\ \mathrm{C} & 0.6809232915 & 1.7668225915 & 1.6258880899 \\ \mathrm{C} & -0.661984807 & 1.5828743627 & 1.2713783064 \\ \mathrm{C} & -1.670424442 & 1.8578657583 & 2.1853969119 \\ \mathrm{C} & -1.3612735859 & 2.3118663502 & 3.4636459767 \\ \mathrm{C} & -0.0309935486 & 2.5357116662 & 3.8080106933 \\ \mathrm{C} & 0.9822739648 & 2.2803067678 & 2.886930518\end{array}$




\begin{tabular}{|c|c|c|c|}
\hline 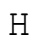 & -2.7053932027 & 1.7075857781 & 1.8984316986 \\
\hline 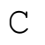 & 1.8451156959 & 3.2190614526 & -0.4647155508 \\
\hline & 2.1171690414 & 4.3758232174 & 0.2805040267 \\
\hline & 1.9547884746 & 5.6323748971 & -0.2881250087 \\
\hline & 1.4998342143 & 5.7543734819 & -1.6003088136 \\
\hline & 1.1924010293 & 4.6137971081 & -2.332429011 \\
\hline & 1.3591148969 & 3.351398664 & -1.7 \\
\hline & 4.39 & -0.5273213265 & -1.4179101892 \\
\hline & 3.6949658455 & -1.6940066998 & -1.8006833267 \\
\hline & 2.7365870363 & -1.6884392879 & -2.8514486952 \\
\hline & 2.4040133817 & -0.4882972913 & -3.5049158479 \\
\hline & 3.0258299246 & 0.7156488178 & -3.0633781346 \\
\hline & 3.9893 & 0.690178078 & -2.0351290537 \\
\hline & 5.5863780611 & -0.5772333582 & -0.5004631517 \\
\hline & 1.3959884918 & -0.4704573808 & -4.6122298497 \\
\hline & -2.1489341209 & 2.5111398535 & 4.1823622549 \\
\hline & -0.9162176439 & 1.2198560978 & 0.2840 \\
\hline & 1.9976025289 & 2.5089197583 & 3.188109235 \\
\hline & 0.373216705 & 3.1252643169 & 5.1256034698 \\
\hline & -3.0284915159 & -1.0867832465 & 3.926222918 \\
\hline & -1.8762815089 & -2.0909352735 & -0.0867552991 \\
\hline & 1.1029941453 & -1.116627023 & 2.8462751112 \\
\hline & -0.557758245 & -0.7280672117 & 4.940233115 \\
\hline & 2.2633618 & 6535194195 & -0.3548557843 \\
\hline & 1.7956738839 & -6.3406262483 & -2.0710579875 \\
\hline & -0.0316978493 & -5.9886527822 & -3.7150844199 \\
\hline & -1.3807382086 & -3.9031982189 & -3.6202974961 \\
\hline & -0.87019 & -2.1739487641 & -1.9583660955 \\
\hline & 2.4524812064 & 4.2999222631 & 1.3103607701 \\
\hline & 2.1743000819 & 6.5185769751 & 0.2979638876 \\
\hline & 1.37 & 6.7370855838 & -2.0417424288 \\
\hline & 0.80515132 & 4.7018017673 & -3.3423661943 \\
\hline & 1.0 & 2.4712482309 & -2.3205212848 \\
\hline & 3.9 & -2.6310136878 & -1.3015410735 \\
\hline & 1549062 & -2.6073641142 & -3.1197198076 \\
\hline & 2.7497692657 & 1.6602041662 & -3.5146825329 \\
\hline & 4.4449920157 & 1.6211292645 & -1.7143972735 \\
\hline & 1.9 & -0.5288659152 & -5.5706263301 \\
\hline & 0.8 & 0.44 & -4.59 \\
\hline & 0.7 & -1.3209393913 & -4.5325136153 \\
\hline & 6.4837354213 & -0.3348735406 & -1.078451795 \\
\hline & 5.51 & 0.1456235026 & 0.3132889752 \\
\hline $\mathrm{H}$ & 5.72 & -1.5751214963 & -0.0806833757 \\
\hline & 2.941696403 & 0.2483233676 & 2.7980788902 \\
\hline & 5.035025003 & 0.2643493038 & 4.0060058923 \\
\hline$t$ & 6.9245387977 & 1.72964254 & 3.2483742634 \\
\hline & 6.6152169673 & 3.1854792283 & 1.2637425561 \\
\hline $\mathrm{H}$ & 26877372 & 3.1858310599 & 0.0438014101 \\
\hline $\mathrm{H}$ & $\$ 2616945$ & -2.3190211227 & 2.8637621935 \\
\hline & 4.52 & -4.3254938926 & 4.1086282693 \\
\hline $\mathrm{H}$ & 2.3102395848 & -5.3118718496 & 3.5954821968 \\
\hline H & 0.8722626096 & -4.3011869591 & 1.8697922743 \\
\hline $\mathrm{H}$ & 3.8678546849 & -1.3262320599 & 1.124211938 \\
\hline 0 & -1.3090016951 & -0.0578363441 & 5.6496601899 \\
\hline $\mathrm{N}$ & 0.6451517881 & -1.1761511429 & 5.3550708532 \\
\hline & 1.2190578514 & -1.7246715311 & 4.7321052646 \\
\hline & 1.2729 & 436946915 & \\
\hline
\end{tabular}




\begin{tabular}{|c|c|c|c|}
\hline $\mathrm{C}$ & 2.7723173615 & -0.7417000927 & 6.3432933728 \\
\hline$H$ & 1.0304710037 & 0.3121593558 & 6.7467595335 \\
\hline $\mathrm{C}$ & 0.8335603591 & -1.5570609489 & 7.8011755746 \\
\hline $\mathrm{H}$ & 1.3089962933 & -1.1721072091 & 8.70487375 \\
\hline$\exists$ & -0.2503486247 & -1.475894084 & 7.904885952 \\
\hline $\mathrm{H}$ & 1.0963074126 & -2.6123640779 & 7.686656926 \\
\hline O & 3.4511915198 & -0.5702916191 & 7.465949864 \\
\hline O & 3.281673055 & -0.8629174005 & 5.2451424003 \\
\hline$f$ & 4.8478914363 & -0.3116572063 & 7.321854499 \\
\hline $\mathrm{H}$ & 5.2467638183 & -0.2672516952 & 8.333405 \\
\hline $\mathrm{H}$ & 5.3321287909 & -1.1081146803 & 6.75293802 \\
\hline 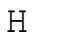 & 4.9886557952 & 0.6469309762 & 6.8171525141 \\
\hline 0 & 1.2328619968 & 3.9944072183 & 5.1990927281 \\
\hline $\mathrm{N}$ & -0.2668796561 & 2.6336085828 & 6.217371029 \\
\hline $\mathrm{H}$ & -0.7848825474 & 1.760944015 & 6.139077752 \\
\hline C & 0.0990657412 & 3.1258889692 & 7.5252620307 \\
\hline C & -0.6305026704 & 2.3284089244 & 8.60682017 \\
\hline $\mathrm{C}$ & 1.6094543132 & 3.0193424614 & 7.7402402468 \\
\hline $\mathrm{H}$ & -0.1595681724 & 4.1859029002 & 7.60400 \\
\hline $\mathrm{H}$ & -0.3817932703 & 2.7171430038 & 9.596213 \\
\hline $\mathrm{H}$ & -1.7111035093 & 2.4050439113 & 8.4649770 \\
\hline $\mathrm{H}$ & -0.3506583875 & 1.2711963152 & 8.56308762 \\
\hline 0 & 2.0487843022 & 4.0169727528 & 8.50355017 \\
\hline 0 & 2.3089402864 & 2.1157248729 & 7.338 \\
\hline $\mathrm{C}$ & 3.4468804753 & 4.0041914883 & 8.7936566675 \\
\hline $\mathrm{H}$ & 3.6200268215 & 4.852688527 & 9.453154487 \\
\hline $\mathrm{H}$ & 3.7298663421 & 3.0706181139 & 9.28540980 \\
\hline $\mathrm{H}$ & 4.0208515786 & 4.1135136345 & 7.870880 \\
\hline & & & \\
\hline $2 \mathrm{~ms}$ & SIIP & & \\
\hline C & 1.040896 & -3.840682 & 0.817914 \\
\hline $\mathrm{C}$ & 1.381924 & -2.497580 & 1.043097 \\
\hline $\mathrm{C}$ & 0.948113 & -1.877534 & 2.209014 \\
\hline $\mathrm{C}$ & 0.190098 & -2.573308 & 3.149094 \\
\hline C & -0.131996 & -3.905014 & 2.926823 \\
\hline $\mathrm{C}$ & 0.296849 & -4.539144 & 1.758621 \\
\hline $\mathrm{P}$ & 2.247993 & -1.544213 & -0.261004 \\
\hline $\mathrm{Ru}$ & 3.018679 & 0.632343 & 0.313680 \\
\hline $\mathrm{Cl}$ & 3.400067 & 0.901381 & -2.054924 \\
\hline $\mathrm{C}$ & 1.080364 & -1.843189 & -1.643943 \\
\hline $\mathrm{C}$ & 1.444146 & -2.099898 & -2.965775 \\
\hline $\mathrm{C}$ & 0.461881 & -2.362358 & -3.921423 \\
\hline $\mathrm{C}$ & -0.882043 & -2.384731 & -3.570366 \\
\hline $\mathrm{C}$ & -1.253011 & -2.142037 & -2.248216 \\
\hline $\mathrm{C}$ & -0.274676 & -1.849433 & -1.306622 \\
\hline $\mathrm{H}$ & 0.756275 & -2.560194 & -4.946532 \\
\hline $\mathrm{C}$ & 3.756054 & -2.545813 & -0.595138 \\
\hline $\mathrm{C}$ & 4.194046 & -3.504918 & 0.325987 \\
\hline $\mathrm{C}$ & 5.418804 & -4.147155 & 0.151968 \\
\hline C & 6.219992 & -3.845643 & -0.944100 \\
\hline C & 5.793845 & -2.890196 & -1.863924 \\
\hline $\mathrm{C}$ & 4.578880 & -2.237533 & -1.687865 \\
\hline $\mathrm{P}$ & 0.963575 & 1.823691 & 0.024362 \\
\hline $\mathrm{C}$ & 0.042912 & 1.895858 & 1.609982 \\
\hline C & -0.769795 & 0.819317 & 1.984539 \\
\hline $\mathrm{C}$ & -1.422092 & 0.810388 & 3.212597 \\
\hline $\mathrm{C}$ & -1.267372 & 1.880249 & 4.090700 \\
\hline
\end{tabular}




\begin{tabular}{|c|c|c|c|}
\hline C & -0.467418 & 2.960794 & 3.728441 \\
\hline $\mathrm{C}$ & 0.182882 & 2.970154 & 2.496585 \\
\hline $\mathrm{C}$ & -0.320520 & 1.398580 & -1.217840 \\
\hline C & 0.058455 & 1.116695 & -2.536868 \\
\hline $\mathrm{C}$ & -0.907119 & 0.929778 & -3.517036 \\
\hline $\mathrm{C}$ & -2.259642 & 1.013012 & -3.201811 \\
\hline $\mathrm{C}$ & -2.641619 & 1.333370 & -1.901887 \\
\hline $\mathrm{C}$ & -1.675417 & 1.541607 & -0.920346 \\
\hline $\mathrm{H}$ & -0.599325 & 0.708773 & -4.533162 \\
\hline $\mathrm{C}$ & 1.223305 & 3.591830 & -0.417077 \\
\hline $\mathrm{C}$ & 0.156489 & 4.491737 & -0.277317 \\
\hline C & 0.301165 & 5.818238 & -0.662145 \\
\hline C & 1.503889 & 6.261809 & -1.210227 \\
\hline $\mathrm{C}$ & 2.552795 & 5.367301 & -1.386529 \\
\hline $\mathrm{C}$ & 2.412532 & 4.036842 & -0.996708 \\
\hline $\mathrm{C}$ & 3.330662 & 0.786821 & 2.607576 \\
\hline $\mathrm{C}$ & 4.051495 & -0.303696 & 2.067114 \\
\hline $\mathrm{C}$ & 5.036695 & -0.131457 & 1.056004 \\
\hline $\mathrm{C}$ & 5.277664 & 1.140548 & 0.506547 \\
\hline C & 4.488441 & 2.234860 & 0.965574 \\
\hline $\mathrm{C}$ & 3.530914 & 2.051708 & 1.983349 \\
\hline $\mathrm{C}$ & 2.488577 & 0.651469 & 3.844793 \\
\hline $\mathrm{C}$ & 6.312157 & 1.333597 & -0.558879 \\
\hline $\mathrm{H}$ & -3.013527 & 0.850995 & -3.964752 \\
\hline $\mathrm{H}$ & 1.106850 & 1.038320 & -2.793724 \\
\hline $\mathrm{H}$ & -2.021461 & 1.828500 & 0.065459 \\
\hline $\mathrm{C}$ & -4.072868 & 1.547071 & -1.510904 \\
\hline $\mathrm{H}$ & -1.653663 & -2.586881 & -4.305428 \\
\hline $\mathrm{H}$ & 2.484771 & -2.108189 & -3.264216 \\
\hline $\mathrm{H}$ & -0.571740 & -1.611396 & -0.291175 \\
\hline $\mathrm{C}$ & -2.700818 & -2.160389 & -1.859413 \\
\hline $\mathrm{H}$ & 3.588911 & -3.755615 & 1.190246 \\
\hline $\mathrm{H}$ & 5.741655 & -4.886287 & 0.877700 \\
\hline $\mathrm{H}$ & 7.171880 & -4.348250 & -1.079587 \\
\hline $\mathrm{H}$ & 6.413487 & -2.641274 & -2.719431 \\
\hline $\mathrm{H}$ & 4.299572 & -1.447446 & -2.374302 \\
\hline $\mathrm{H}$ & -0.793922 & 4.158679 & 0.128381 \\
\hline $\mathrm{H}$ & -0.530411 & 6.504804 & -0.543198 \\
\hline $\mathrm{H}$ & 1.613900 & 7.297841 & -1.513288 \\
\hline $\mathrm{H}$ & 3.479908 & 5.697145 & -1.844070 \\
\hline $\mathrm{H}$ & 3.210866 & 3.332897 & -1.194772 \\
\hline $\mathrm{H}$ & 3.873490 & -1.298598 & 2.463970 \\
\hline $\mathrm{H}$ & 5.573429 & -0.993157 & 0.677494 \\
\hline $\mathrm{H}$ & 4.618104 & 3.214292 & 0.522889 \\
\hline $\mathrm{H}$ & 2.940007 & 2.900151 & 2.312201 \\
\hline $\mathrm{H}$ & 7.246574 & 1.658928 & -0.089297 \\
\hline $\mathrm{H}$ & 5.998874 & 2.090225 & -1.279335 \\
\hline $\mathrm{H}$ & 6.495044 & 0.401283 & -1.095464 \\
\hline $\mathrm{H}$ & 2.971780 & 1.202180 & 4.657992 \\
\hline $\mathrm{H}$ & 1.488612 & 1.067208 & 3.712794 \\
\hline $\mathrm{H}$ & 2.403942 & -0.391276 & 4.154054 \\
\hline $\mathrm{H}$ & -0.932459 & -0.017823 & 1.314018 \\
\hline $\mathrm{H}$ & -2.060752 & -0.033586 & 3.451323 \\
\hline $\mathrm{H}$ & -1.778605 & 1.880294 & 5.047822 \\
\hline $\mathrm{H}$ & -0.353310 & 3.804804 & 4.400753 \\
\hline $\mathrm{H}$ & 0.780818 & 3.833242 & 2.221560 \\
\hline $\mathrm{H}$ & -0.164014 & -2.061005 & 4.037418 \\
\hline
\end{tabular}




\begin{tabular}{|c|c|c|c|}
\hline $\mathrm{H}$ & -0726721 & (15 150000 & 3651501 \\
\hline$\Pi$ & -0.726721 & -4.450923 & 3.651581 \\
\hline $\mathrm{H}$ & 0.044196 & -5.578960 & 1.579090 \\
\hline $\mathrm{H}$ & 1.350137 & -4.334889 & -0.098430 \\
\hline $\mathrm{H}$ & 1.160700 & -0.829364 & 2.362307 \\
\hline 0 & -3.592940 & -1.872041 & -2.658060 \\
\hline $\mathrm{N}$ & -2.940765 & -2.512421 & -0.579428 \\
\hline $\mathrm{H}$ & -2.170880 & -2.742042 & 0.031211 \\
\hline $\mathrm{C}$ & -4.236568 & -2.382587 & 0.050443 \\
\hline $\mathrm{C}$ & -3.991056 & -2.062849 & 1.514596 \\
\hline $\mathrm{H}$ & -4.724025 & -1.481840 & -0.338136 \\
\hline $\mathrm{C}$ & -5.142266 & -3.590467 & -0.185437 \\
\hline $\mathrm{H}$ & -6.115586 & -3.429867 & 0.281205 \\
\hline $\mathrm{H}$ & -5.278504 & -3.722440 & -1.260715 \\
\hline $\mathrm{H}$ & -4.700073 & -4.502067 & 0.225982 \\
\hline 0 & -5.103125 & -2.136675 & 2.227661 \\
\hline 0 & -2.904454 & -1.756314 & 1.967811 \\
\hline $\mathrm{C}$ & -5.031379 & -1.622829 & 3.557789 \\
\hline $\mathrm{H}$ & -6.001355 & -1.827126 & 4.006918 \\
\hline $\mathrm{H}$ & -4.236204 & -2.116111 & 4.120698 \\
\hline $\mathrm{H}$ & -4.850658 & -0.546069 & 3.520951 \\
\hline 0 & -4.405032 & 2.479197 & -0.789372 \\
\hline $\mathrm{N}$ & -4.962985 & 0.646585 & -2.001027 \\
\hline $\mathrm{H}$ & -4.621269 & -0.231825 & -2.385249 \\
\hline $\mathrm{C}$ & -6.354997 & 0.765838 & -1.633718 \\
\hline $\mathrm{C}$ & -7.140673 & -0.432812 & -2.166599 \\
\hline $\mathrm{C}$ & -6.508598 & 0.845438 & -0.114207 \\
\hline $\mathrm{H}$ & -6.764641 & 1.693517 & -2.043992 \\
\hline $\mathrm{H}$ & -8.199136 & -0.331856 & -1.918947 \\
\hline $\mathrm{H}$ & -7.042267 & -0.493056 & -3.253051 \\
\hline $\mathrm{H}$ & -6.764909 & -1.365171 & -1.733665 \\
\hline 0 & -7.540861 & 1.617774 & 0.215718 \\
\hline 0 & -5.836544 & 0.235192 & 0.687560 \\
\hline $\mathrm{C}$ & -7.796445 & 1.743955 & 1.614916 \\
\hline $\mathrm{H}$ & -8.686454 & 2.365075 & 1.698327 \\
\hline $\mathrm{H}$ & -7.967880 & 0.763519 & 2.065267 \\
\hline $\mathrm{H}$ & -6.947840 & 2.223278 & 2.107895 \\
\hline & 20 & & \\
\hline \multicolumn{4}{|c|}{$2 \mathrm{mAa} I \mathrm{p}$} \\
\hline C & -3.6934455513 & -1.3068831992 & -1.8976018573 \\
\hline $\mathrm{C}$ & -3.5264916697 & -0.05458643 & -2.5113574516 \\
\hline $\mathrm{C}$ & -4.2360858971 & 1.0483228429 & -1.9476295138 \\
\hline $\mathrm{C}$ & -5.0957920456 & 0.8808586788 & -0.8587865003 \\
\hline $\mathrm{C}$ & -5.3434077961 & -0.4199750075 & -0.3077577094 \\
\hline $\mathrm{C}$ & -4.6098430979 & -1.4965877541 & -0.8221641436 \\
\hline $\mathrm{Ru}$ & -3.0578428417 & 0.0850950929 & -0.2207708177 \\
\hline $\mathrm{Cl}$ & -3.5731237386 & 0.8440835587 & 1.9980463748 \\
\hline $\mathrm{C}$ & -2.7648057131 & 0.0899184354 & -3.7965051489 \\
\hline $\mathrm{C}$ & -6.3461840405 & -0.5915081439 & 0.7913805532 \\
\hline $\mathrm{P}$ & -1.6231030609 & -1.6061300058 & 0.6737352158 \\
\hline C & -2.241564517 & -2.4366591675 & 2.1872289204 \\
\hline C & -3.5928972779 & -2.3997034668 & 2.533532923 \\
\hline $\mathrm{C}$ & -4.0654446978 & -3.1311777871 & 3.6188816725 \\
\hline $\mathrm{C}$ & -3.1893922664 & -3.9059882732 & 4.3717050626 \\
\hline $\mathrm{C}$ & -1.838287218 & -3.9485782074 & 4.0347532496 \\
\hline C & -1.3651584831 & -3.2212125676 & 2.9482804653 \\
\hline C & 0.1057582554 & -1.2267753334 & 1.1435513464 \\
\hline$C$ & 0.3045637011 & -0.4072777051 & 2.2601045411 \\
\hline
\end{tabular}




\begin{tabular}{|c|c|c|c|}
\hline 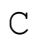 & 1.5896644871 & -0.0732627358 & 2.6646122526 \\
\hline  & 2.696282121 & -0.5700357986 & 1.9802123196 \\
\hline & 2.5072348185 & -1.4137566743 & 0.8854419581 \\
\hline & 1.21336128 & -1.7373093767 & 0.476239279 \\
\hline & 3.6148299349 & -2.0310793591 & 0.0732232635 \\
\hline & 3.3620257067 & -2.7906431686 & -0.860 \\
\hline$\rangle_{T}$ & 4.8880757287 & -1.7263807473 & 0.4114112633 \\
\hline C & 5.9899345111 & -2.3633309538 & -0.2826214324 \\
\hline$c$ & 5.8562142267 & -2.1264086795 & -1.7893747245 \\
\hline & 5.5928327223 & -1.0403011712 & -2.2777854042 \\
\hline & 6.1420 & -3.1903470329 & -2.5165120795 \\
\hline & 6.045 & -3.027188975 & -3.9349035436 \\
\hline & -1.4989962487 & -3.0121667052 & -0.506493453 \\
\hline & -0.8268801556 & -2.8503586388 & -1.726202053 \\
\hline & -0.8075145255 & -3.8703995233 & -2.6687559953 \\
\hline & -1.4781643021 & -5.0665783397 & -2.4 \\
\hline & -2.153119684 & -5.2354500901 & -1.2145492447 \\
\hline & -2.1603162796 & -4.218283348 & -0.2602159124 \\
\hline & -1.3503248884 & 1.7913350141 & -0.2822218678 \\
\hline $\mathrm{C}$ & -1.9403747987 & 3.0803543715 & -1.4562652191 \\
\hline & -3.0074788821 & 3.883039654 & -1.0270576144 \\
\hline 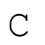 & -3.5444424202 & 6726657 & -1.8716246752 \\
\hline & -3.0213962682 & 0387691 & -3.1544079298 \\
\hline & -1.9506473686 & 4.2431840851 & -3.5763383495 \\
\hline & -1.4052790837 & 3.2776631333 & -2.7275503802 \\
\hline $\mathrm{C}$ & 0.2731201473 & 1.2733498827 & -0.9640424927 \\
\hline $\mathrm{C}$ & 1.477856044 & 1.6557373222 & -0.3832764784 \\
\hline  & 2.6972250117 & 1.2680620315 & -0.9408002194 \\
\hline $\mathrm{C}$ & 2.716803427 & 0.4915885494 & -2.0983512491 \\
\hline C & 1.5143831205 & 0.0957997709 & -2.6790283817 \\
\hline $\mathrm{C}$ & 0.3054384951 & 0.4785286656 & -2.1138425257 \\
\hline $\mathrm{C}$ & 3.9271147227 & 316 & 16864 \\
\hline 0 & 3.83 & 2.52421 & 0.731 \\
\hline N & 5.1224274442 & 1.2650293644 & -0.629746485 \\
\hline $\mathrm{C}$ & 6.3400972532 & 1.7237410533 & 0.0102756284 \\
\hline $\mathrm{C}$ & 6.2412383343 & 1.4952832293 & 1.5219064976 \\
\hline 0 & 5.84017 & 626165 & 2.0178750385 \\
\hline 0 & 6.71 & 223013 & 2.237 \\
\hline $\mathrm{C}$ & 6.6427594544 & 2.3448201839 & 3.6592347807 \\
\hline $\mathrm{C}$ & -0.8443924866 & 2.9065890329 & 1.1019722128 \\
\hline $\mathrm{C}$ & -0.2085329678 & 4.1034588803 & 0.7294782676 \\
\hline $\mathrm{C}$ & 0.2929519815 & 4.9725465479 & 1.6884559863 \\
\hline $\mathrm{C}$ & 0.16 & 4.6695840383 & 22285 \\
\hline$C$ & -0.4704549432 & 3.4936016921 & 3.4214808728 \\
\hline $\mathrm{C}$ & -0.9687273534 & 2.6165847922 & 2.4604885327 \\
\hline $\mathrm{H}$ & 6.8891708607 & -1.8066924463 & 0.0094449983 \\
\hline $\mathrm{C}$ & 6.160 & -3.8293469802 & 0.1103730061 \\
\hline $\mathrm{C}$ & 6.7104496727 & 3.1510449718 & -0.3865134103 \\
\hline $\mathrm{H}$ & 7.130074888 & 1.0410139104 & -0.3260077344 \\
\hline H & 5.1948327493 & 0.4675090827 & -1.258634699 \\
\hline $\mathrm{H}$ & 5.1162568866 & -0.9747012145 & 1.0584748208 \\
\hline $\mathrm{H}$ & 3.68789 & -0.2827640833 & 2.3133625257 \\
\hline $\mathrm{H}$ & 1.7308854538 & 0.5818094449 & 3.5173405894 \\
\hline $\mathrm{H}$ & -0.5484279457 & -0.0521967401 & 2.8264914755 \\
\hline 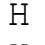 & 1.127638006 & -2.4111017278 & -0.3654877673 \\
\hline H & 1.5158258328 & 2.2508688686 & 0.5201016478 \\
\hline & -0.625940322 & & \\
\hline
\end{tabular}




\begin{tabular}{|c|c|c|c|}
\hline & 1.5252485185 & -0.5215900425 & -3.5715456854 \\
\hline & 3.648114258 & 0.1691557357 & -2.5500300247 \\
\hline & -0.0859309906 & 4.3576678591 & -0.3178962324 \\
\hline & 0.7881139872 & 5.885242684 & 1.3750405606 \\
\hline & 0.5486487107 & 5.3515700131 & 3.792268364 \\
\hline & -0.5943208681 & 3.2539096065 & 4.4725345456 \\
\hline & -1.4923087214 & 1.7296951154 & $2.77^{\circ}$ \\
\hline & -3.4161040824 & 3.7481373775 & -0.0283137596 \\
\hline & -4.3664309986 & 5.4658072255 & 813361 \\
\hline & -3.4397147595 & 5.7751726553 & -3.8138650593 \\
\hline & 3280869 & 4.3918842587 & -4.56 \\
\hline & -0.5 & 2.6954195852 & -3.0 \\
\hline & -0.3168236141 & -1.9193914055 & -1.9433 \\
\hline & -0.2681011444 & -3.7315520458 & -3.6000828818 \\
\hline & -1.4687459532 & -5.8618499241 & -3.1554977521 \\
\hline & -4.2 & 662913 & 7605 \\
\hline & -5.1169160168 & -3.0814189268 & 2938 \\
\hline & -3.5556675142 & -4.4709497069 & 5.2225554497 \\
\hline & -1.1500452435 & -4.5510153534 & 4.61819 \\
\hline & -0.3116415762 & -3.2715127597 & 2.6927248512 \\
\hline & -2 & -6 & 298 \\
\hline & -2.6828284345 & -4.3761861298 & 1783 \\
\hline & 5.6 & 2.2438570436 & 3.970 \\
\hline & 536 & 1.4633121513 & 3.9753918703 \\
\hline & & 3.2503763402 & 4.0732631356 \\
\hline $\mathrm{H}$ & 68 & -3.9848157998 & -4.3607671507 \\
\hline $\mathrm{H}$ & 5.0 & -2.7821621646 & -4.2 \\
\hline & 6.712018956 & -2.2328032279 & -4.2750296228 \\
\hline & -4.0919973311 & 2.0371326659 & -2.363946882 \\
\hline 1 & -5.583289 & 1.7433030101 & -0.4182873364 \\
\hline & 3756 & -2.4873590584 & 9971879661 \\
\hline & -3.1 & -2.160 & 6893 \\
\hline H & -7.3 & -0.216 & 9149 \\
\hline $\mathrm{H}$ & -6.458766 & -1.6411201558 & 1.0692522802 \\
\hline 1 & -6.0408438988 & -0.0168728504 & 1.6713737927 \\
\hline & -3.4 & 0.0239734947 & 59923 \\
\hline & -2 & 771 & 16842 \\
\hline & -2.2 & 3795 & 29044 \\
\hline $\mathrm{H}$ & 6.2858674769 & -3.8947684894 & 7944 \\
\hline $\mathrm{H}$ & 5.280857674 & -4.4023933399 & -0.18462626 \\
\hline & 7.03 & -4.2620279292 & 376553 \\
\hline $\mathrm{H}$ & 6.7977445742 & 3.2076457017 & 786881 \\
\hline $\mathrm{H}$ & 5.9407192 & .8471294256 & .051 \\
\hline & 7.6627773113 & 3.4427133197 & 0.0595622209 \\
\hline & & & \\
\hline & aI Ip & & \\
\hline C & -0.0706 & $-2 . \varepsilon$ & 4313 \\
\hline$C$ & -0.1 & -2.9411 & 58003 \\
\hline C & -1.0673932925 & -3.8610455267 & -0.0948062655 \\
\hline $\mathrm{C}$ & -1.8595593102 & -4.6677675075 & 0.7119586622 \\
\hline$C$ & -1.7631357597 & -4.5768217578 & 2.0984375766 \\
\hline C & -0.8616691577 & -3.6854179117 & 2.6658953733 \\
\hline $\mathrm{P}$ & 0.6316376457 & -1.7345112377 & -0.6820047861 \\
\hline C & -0.796747419 & -0.6432060198 & -1.0652228023 \\
\hline $\mathrm{C}$ & -2.0310799161 & -0.8379252856 & -0.4474441671 \\
\hline & 167541103 & -0.0164494389 & -0.7454334977 \\
\hline $\mathrm{C}$ & -2. & & \\
\hline
\end{tabular}




\begin{tabular}{|c|c|c|c|}
\hline$C$ & -1.7471134921 & 1.2331336301 & -2.2727636446 \\
\hline C & -0.6697590933 & 0.400517072 & -1.9869541618 \\
\hline & -4.4265819241 & -0.2363425569 & 52 \\
\hline & -4.7817128422 & -1.5234443085 & 0.125171042 \\
\hline & -5.9824391796 & -1.9143618181 & 0.8349589497 \\
\hline & -5.8418476858 & -3.3980752365 & 7942 \\
\hline & -6.7743643692 & 8204 & 3749 \\
\hline & -6.7668133134 & -5.2144146585 & 2.2843581838 \\
\hline & 2.7768146135 & -0.6906080444 & 3460 \\
\hline & 1.9526393253 & 1.1282696582 & 0.9760064871 \\
\hline & 2.3126550978 & 2.7171571423 & 0.1189697948 \\
\hline & 1.6635700245 & 3.0223075148 & -1.0860783187 \\
\hline & 1.9720720936 & 4.1803448347 & -1.787160097 \\
\hline & 2.9547616381 & 5.0454090537 & -1.3064523591 \\
\hline & 3.6135354134 & 4.7459941824 & -0.1201715263 \\
\hline & 3.2920434427 & 3.591361648 & 0.5943721721 \\
\hline & 3.8880220829 & 0.7817958946 & -1.6791550484 \\
\hline & 3.2948749711 & -0.1430631881 & -2.5543708227 \\
\hline & 3.5812714556 & -1.5228165406 & -2.331029258 \\
\hline & 4.4305016471 & -1.9359406854 & -1.2998717583 \\
\hline & 5.0939555714 & -0.9794992328 & -0.4635138164 \\
\hline & 9342875 & 0.3748939195 & -0.6538130564 \\
\hline & 2.5090634047 & 0.3046715491 & -3.7522083412 \\
\hline & 6.071017207 & -1.4365140653 & 0.5749023288 \\
\hline 1 & 2.9725598189 & -2.0735968847 & 1.607601428 \\
\hline & 2.7920541526 & 1.3603451462 & 2.5896055344 \\
\hline 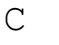 & 4.0300538207 & 0.7735514023 & 2.855 \\
\hline , & 4.7012021425 & 1.0480468717 & 4.043 \\
\hline 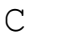 & 4.1404712779 & 1.9126579553 & 4.977 \\
\hline $\mathrm{C}$ & 2.9055284387 & 2.5045268124 & 4.7205297445 \\
\hline C & 2.2337285617 & 2.2327441378 & 3.5339945526 \\
\hline c & 0.1883935182 & 1.2817704983 & 1.4537388039 \\
\hline  & -0.3291127276 & 0.397840226 & 2.407 \\
\hline 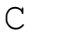 & -1.6590346659 & 0.487447622 & 2.8005770158 \\
\hline 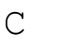 & -2.4893688045 & 1.4686002642 & 2.259761993 \\
\hline C & -1.9696826984 & 2.3779381454 & 1.3415926126 \\
\hline$C$ & -0.6347019215 & 2.2855569678 & 0.954660578 \\
\hline C & -2.7440264186 & 3.5259929123 & 948176 \\
\hline${ }^{-}$ & -4.0800120407 & 3.3501551758 & 0.6177936947 \\
\hline 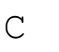 & -4.8682104204 & 4.41342222 & 0.0406371086 \\
\hline$C$ & -4.4547528263 & 4.7014047853 & -1.4022869389 \\
\hline 0 & -4.6738775123 & 5.9800073512 & -1.7061274911 \\
\hline 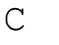 & -4.3814566063 & 6.3560703755 & -3.052829018 \\
\hline 0 & -2.1732654994 & 994287334 & 0.4216218366 \\
\hline 0 & -4.0463275138 & 3.8823679719 & -2.1959313684 \\
\hline $\mathrm{C}$ & 0.7868158161 & -2.813896304 & -2.1623691464 \\
\hline $\mathrm{C}$ & 1.5613948829 & -3.9740444449 & -2.0130915237 \\
\hline $\mathrm{C}$ & 1.7847283018 & -4.8134878873 & -3.0960411049 \\
\hline C & 1.2365767282 & -4.5065029673 & -4.3432645049 \\
\hline $\mathrm{C}$ & 0.451025085 & -3.3696629548 & -4.4911453823 \\
\hline C & 0.2186355242 & -2.5268292456 & -3.4017886673 \\
\hline 0 & -5.1318449781 & 0.6953009453 & 0.347222018 \\
\hline 0 & -4.9987670103 & -4.1121058328 & 0.6377710656 \\
\hline $\mathrm{C}$ & -6.3515809802 & 4.040420349 & 0.0797892981 \\
\hline $\mathrm{H}$ & -6.0344950901 & -1.3663987893 & 1.7815665825 \\
\hline $\mathrm{H}$ & -4.6956456339 & 5.332679606 & 0.6063382534 \\
\hline & -7.262678808 & -1.643124654 & 0.032946982 \\
\hline
\end{tabular}




\begin{tabular}{|c|c|c|c|}
\hline $\mathrm{H}$ & -4.2228714519 & -2.281737408 & -0.2454560496 \\
\hline H & -4.4652441682 & 2.4042772628 & 0.596547405 \\
\hline & -3.5269989809 & 1.5384943352 & 2.5695090606 \\
\hline & -2.0452430641 & -0.2035500746 & 3.5426384778 \\
\hline & 0.3198888314 & -0.3358355744 & 2.8716064112 \\
\hline & -0.2736058469 & 3.0469222873 & 0.2770724944 \\
\hline 4 & -2.1480072732 & -1.609192989 & 0.3030843065 \\
\hline & 0.2834382228 & 0.5650863599 & -2.4769190136 \\
\hline & -1.6380685537 & 2.0550618994 & -2.972409851 \\
\hline & -3.7927965201 & 1.7190454454 & -1.8437075696 \\
\hline & -1.1779968859 & -3.935950084 & -1.1710342679 \\
\hline & -2.569989964 & -5.3489978708 & 0.25847 \\
\hline & -2.3876441666 & -5.2018908203 & 2.7280919602 \\
\hline & -0.7605860503 & -3.6182863022 & 3.7442920182 \\
\hline $\mathrm{H}$ & 0.6445925887 & -2.2089311165 & 2.3154997227 \\
\hline $\mathrm{H}$ & 1.9915236115 & -4.2125285042 & -1.0431609451 \\
\hline $\mathrm{H}$ & 2.3818030823 & -5.7106709248 & -2.9694249185 \\
\hline $\mathrm{H}$ & 1.4126718207 & -5.160986298 & -5.1903890219 \\
\hline & 0.0029493814 & -3.1388077479 & -5.4520646003 \\
\hline $\mathrm{H}$ & -0.4181917077 & -1.6569390681 & 5264774958 \\
\hline $\mathrm{H}$ & 0.9120651397 & 2.3473 & -1.48 \\
\hline $\mathrm{H}$ & 802894 & 4.40 & -2.7072839439 \\
\hline $\mathrm{H}$ & 3.1985481734 & 5.9507409505 & -1.8520943078 \\
\hline $\mathrm{H}$ & 4.4548820084 & 0.070418181 & 2.152988898 \\
\hline $\mathrm{H}$ & 5.6557919478 & 0.5720595455 & 4.2426668974 \\
\hline $\mathrm{H}$ & 4.6 & 2.1216809714 & 5.9071261366 \\
\hline $\mathrm{H}$ & 2.4630354948 & 3.1811449304 & 5.443 \\
\hline $\mathrm{H}$ & 1.277163024 & 2.7085063836 & 3.3424146867 \\
\hline $\mathrm{H}$ & 4.3763439962 & 5.4159460346 & 0.2625799171 \\
\hline $\mathrm{H}$ & 3.8077155155 & 3.3824022482 & 1.5253526744 \\
\hline $\mathrm{H}$ & -6.9072895661 & -5.7971507198 & 1.372123957 \\
\hline $\mathrm{H}$ & -5.8206437403 & -5.4961917286 & 2.7506406139 \\
\hline $\mathrm{H}$ & -7.5942685861 & -5.3715758868 & 2.9730560124 \\
\hline $\mathrm{H}$ & -4.6109865353 & 7.4179719138 & -3.120878266 \\
\hline $\mathrm{H}$ & -3.3270842793 & 6.1773819228 & -3.2736639121 \\
\hline $\mathrm{H}$ & -4.9957774554 & 5.7844751827 & -3.7521393266 \\
\hline $\mathrm{H}$ & 3727545 & -2.2672562533 & -2.9620698378 \\
\hline $\mathrm{H}$ & 4.58 & -2.993964602 & -1.1224720205 \\
\hline $\mathrm{H}$ & 5.2327954515 & 1.1270641361 & -0.0105207428 \\
\hline $\mathrm{H}$ & 3.6822953173 & 1.8361752178 & -1.806635376 \\
\hline $\mathrm{H}$ & 6.9009996727 & -1.9551693758 & 0.0858118881 \\
\hline $\mathrm{H}$ & 6.4769978289 & -0.5944986724 & 1.1384830542 \\
\hline $\mathrm{H}$ & 5.5 & -2.1310 & 1.2685151365 \\
\hline $\mathrm{H}$ & 3.181996 & 0.3713283565 & -4.61348 \\
\hline $\mathrm{H}$ & 2.0754252522 & 1.2949044899 & -3.5937552228 \\
\hline $\mathrm{H}$ & 1.7210968835 & -0.4080147571 & -4.0036589646 \\
\hline $\mathrm{H}$ & -6.9564738992 & 4.8411085946 & -0.3504104057 \\
\hline $\mathrm{H}$ & -6.5289810018 & 3.1189865922 & -0.4821765013 \\
\hline $\mathrm{H}$ & -6.6660226031 & 3.8814088652 & 1.1136929832 \\
\hline $\mathrm{H}$ & -8.1391320815 & -1.9586513964 & 0.602130583 \\
\hline $\mathrm{H}$ & -7.3348195497 & -0.5736646677 & -0.168040633 \\
\hline $\mathrm{H}$ & -7.2392820015 & -2.1846262006 & -0.9166552677 \\
\hline & & & \\
\hline \multicolumn{4}{|c|}{$2 \mathrm{pAaIp}$} \\
\hline $\mathrm{C}$ & 7.207 & -1.7 & -3 . \\
\hline 0 & 7.21 & -0.4043785429 & -3.050498414 \\
\hline $\mathrm{C}$ & 7.8798850584 & 1.4777089364 & 3.114772877 \\
\hline
\end{tabular}




\begin{tabular}{|c|c|}
\hline & 7.5643247983 \\
\hline & 6.4050690685 \\
\hline & 6.1711329602 \\
\hline & 6.2536507071 \\
\hline & 6.3561312233 \\
\hline & 5.4210448613 \\
\hline & 5.6434848373 \\
\hline & 5.1526824481 \\
\hline & 4.0424955501 \\
\hline & 4.8667849674 \\
\hline & 3.8025939955 \\
\hline & 4.1106568978 \\
\hline & 3.9487874016 \\
\hline & 7.2945299673 \\
\hline & 6.1477071134 \\
\hline & 6.3965826511 \\
\hline & 7.6264238543 \\
\hline & 5.1031595504 \\
\hline & 4.7712939339 \\
\hline & 2.4135145392 \\
\hline & 2.0881863384 \\
\hline & 2.8584618325 \\
\hline & 0.7541117204 \\
\hline & 0.5193280783 \\
\hline & -0.2772505142 \\
\hline & -0.6923200046 \\
\hline & 0.060405475 \\
\hline & -2.015256042 \\
\hline & 1.3880552712 \\
\hline & 1.6584247491 \\
\hline & 2.7053895909 \\
\hline & 1.6621390594 \\
\hline & 1.8909976894 \\
\hline & 0.3589099717 \\
\hline & 0.0516083444 \\
\hline & -1.7128985068 \\
\hline & -0.4125986935 \\
\hline & 1.1034337901 \\
\hline & 0.9114538246 \\
\hline & 2.4225820491 \\
\hline & 3.206985952 \\
\hline & -1.6517100044 \\
\hline & -2.372560053 \\
\hline & -2.7551291893 \\
\hline & -2.6373210215 \\
\hline & -3.3002376068 \\
\hline & -3.5945246205 \\
\hline & -3.462059588 \\
\hline & -3.8809434457 \\
\hline & -3.0918487052 \\
\hline & -3.2251773178 \\
\hline & -2.5648254756 \\
\hline & -2.3488407256 \\
\hline & -1.8134835985 \\
\hline & -1.0418377586 \\
\hline & -0.5354962265 \\
\hline & -0.9250353838 \\
\hline
\end{tabular}

\begin{tabular}{|c|c|}
\hline 0.156 & \\
\hline 437583 & 2.6610074193 \\
\hline $\begin{array}{l}0150910651 \\
4363257288\end{array}$ & 2.0283960756 \\
\hline $\begin{array}{l}.4363257288 \\
.1189354034\end{array}$ & $\begin{array}{l}1.6111065952 \\
-2.176347665\end{array}$ \\
\hline .3292669275 & -1.6935943089 \\
\hline-0.9254640237 & -1.8096420668 \\
\hline 0.9387126241 & 1.8190697664 \\
\hline 1.688 & -0.9858978814 \\
\hline 1.9098 & -1.7408028643 \\
\hline-1.5699482422 & 1.0090244461 \\
\hline-1.67027089 & 1.8491962408 \\
\hline 2.0096522328 & -2.9598250873 \\
\hline-1.7776525332 & 3.0611232077 \\
\hline-1.9860611674 & 0.7309518179 \\
\hline-2.002797745 & 2.54635752 \\
\hline 1.9389669971 & -2.599932618 \\
\hline 1.5715000001 & -0.8780888392 \\
\hline 1.5000017708 & 0.0094213805 \\
\hline-1.2916501524 & 0.0366692638 \\
\hline-1.6537530789 & 1.2670067145 \\
\hline-1.6 & -0.0906824719 \\
\hline-1.5804673549 & -0.8559339069 \\
\hline-1.5833532505 & -0.4896152976 \\
\hline-1.544106 & -1.5482601252 \\
\hline-1.6216835722 & 0.4596460538 \\
\hline-1.6464342611 & 2.5862951468 \\
\hline 1.6327420 & 1.8148285421 \\
\hline-1.7344292055 & -0.1329136771 \\
\hline-1.6529934842 & 2.2091751481 \\
\hline-1.6794085124 & 3.258437255 \\
\hline 1.9998606143 & -1.0612044053 \\
\hline 2.4764999728 & -1.8535608264 \\
\hline 2.829811627 & -2.8520062353 \\
\hline 2.4525067352 & -1.3956974351 \\
\hline 1.9189318154 & -0.1374199079 \\
\hline 1.7788493192 & 0.3722873056 \\
\hline 2.821239805 & -2.0522865224 \\
\hline 1.4783029991 & 0.6702108065 \\
\hline 1.0439105422 & 1.643732451 \\
\hline 1.5325318878 & 0.2200714763 \\
\hline .152293 & 0.8686571248 \\
\hline 1.7145298103 & 2.1978342141 \\
\hline 3.4672546311 & 0.0214344636 \\
\hline 4.3445406371 & 1.038318577 \\
\hline 4.0671174348 & 2.0796454245 \\
\hline 5.5914388625 & 0.7291078982 \\
\hline 6.2592633788 & 1.5319595762 \\
\hline 5.9791893163 & -0.5951794002 \\
\hline 6.951444673 & -0.832133037 \\
\hline 5.1063668449 & -1.6176711025 \\
\hline 5.3926890575 & -2.6561689871 \\
\hline 3.858236468 & -1.312895675 \\
\hline 3.164282433 & -2.1193753535 \\
\hline-2.9405387962 & -1.5081127646 \\
\hline-4.0872454615 & -1.2672945535 \\
\hline-4.215 & $-0.3154 \varepsilon$ \\
\hline-5.0730085263 & -2.237853897 \\
\hline
\end{tabular}




\begin{tabular}{|c|c|c|c|}
\hline $\mathrm{H}$ & -0.3241629582 & -5.9541030438 & -2.0385181841 \\
\hline & -1.57845431 & -4.9306690594 & -3.4614869801 \\
\hline & -1.4837590694 & -5.6991569551 & 221609569 \\
\hline & -3.2315586116 & 0.2256210384 & -0.681267334 \\
\hline & -2.9212052315 & -2.8032693332 & 1.0834316269 \\
\hline & -2.344904337 & -3.7980858351 & -3.707394874 \\
\hline & -2.4616045316 & -2.8068831327 & -2.734272434 \\
\hline & -2.8431202974 & -3.6722036262 & \\
\hline & -3.0232444117 & -1.9095337073 & -2 \\
\hline & 6.2627240218 & -1.93 & -4.0 \\
\hline & 7.3493719752 & -2.4642013835 & -2.77497572 \\
\hline & 8.0369459599 & -1.7743387231 & -4.280739079 \\
\hline & 8.8557970305 & 1.398070483 & 3.589206 \\
\hline & 7.1298685508 & 1.8198829435 & 3.83 \\
\hline & 7.97 & 2.1707203377 & 2.27 \\
\hline & -5.2076173559 & -0.2839264437 & 0.4257022637 \\
\hline & -5.0009124868 & 1.1108235989 & 0.390440765 \\
\hline & -4.9170316319 & 1.8167342765 & -0.844 \\
\hline & -4.8 & 609 & -2.0 \\
\hline & -4 & 9194 & $-2.02 \varepsilon$ \\
\hline & -5.1383854967 & -0.9820924685 & -0.818599856 \\
\hline & -4 & 1.6716185651 & 1.3198458656 \\
\hline $\mathrm{H}$ & -4 & 2.8941 & -0.8326221414 \\
\hline & -4 & 984 & -3.3 \\
\hline $\mathrm{H}$ & $-4 . \varepsilon$ & 9411 & -2 \\
\hline $\mathrm{H}$ & -5.213536 & -2.0645984133 & $-0.81 \mathrm{~s}-5$ \\
\hline & -5.659833 & 1.6648712438 & -3.984137658 \\
\hline & -3 & 1.4 & 3898 \\
\hline $\mathrm{H}$ & -4 . & 142 & 0923 \\
\hline$C$ & -5 & 377 & 1.68 \\
\hline $\mathrm{H}$ & -6 & 3879 & 1.8 \\
\hline $\mathrm{H}$ & -5.4786721422 & -2.0497674775 & 1.640525076 \\
\hline$\Pi$ & -5.151773394 & -0.5726829257 & 2.5703836581 \\
\hline C & -2.9703388848 & -2.5422846363 & 2.4584255241 \\
\hline c & -3.6 & -3 & 3.32 \\
\hline C & -4.2 & 4463 & 287 \\
\hline C & -4.4049233326 & -4.6959599472 & 1.4616 \\
\hline $\mathrm{C}$ & -3.6631 & -3.891 & 06609304 \\
\hline $\mathrm{H}$ & -2.47 & 380574 & 170638 \\
\hline $\mathrm{H}$ & -3 & 6468 & 568 \\
\hline $\mathrm{H}$ & -4 & -5.0 & 3.502 \\
\hline $\mathrm{H}$ & -4. & -5.5317 & 92947 \\
\hline $\mathrm{H}$ & -3.6573232149 & -4.131054013 & -0.4567097665 \\
\hline $\mathrm{C}$ & -2. & 0.8 & $2.8^{\circ}$ \\
\hline $\mathrm{C}$ & -2.528 & 285 & 3368 \\
\hline C & -1.6561 & 34138 & 4.9 \\
\hline C & -0 & 2.4 & 4.3 \\
\hline $\mathrm{C}$ & -0.786254 & 2.5313858327 & 2.93895866 \\
\hline $\mathrm{H}$ & -3.2 & 7874 & 328 \\
\hline $\mathrm{H}$ & 90985 & 1.5746773844 & 226 \\
\hline $\mathrm{H}$ & -0.11 & 276 & 3585 \\
\hline $\mathrm{H}$ & -0.1080364655 & 7488 & 2.4 \\
\hline $\mathrm{Cl}$ & -1.759110824 & 0.3853625889 & -2.5720141173 \\
\hline $\mathrm{H}$ & -3.1936687546 & 0.2405096485 & 2.3053140746 \\
\hline $\mathrm{H}$ & & -3.0307839828 & 0.4933156707 \\
\hline $\mathrm{H}$ & 7.3887222751 & -1.4369871002 & -0.2074113781 \\
\hline 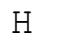 & 8.2455507044 & -1.9278751085 & 1.2622020759 \\
\hline
\end{tabular}




\begin{tabular}{|c|c|c|c|}
\hline $\mathrm{H}$ & 7.6531350711 & 2.6139478148 & 536616711 \\
\hline T & 7.6819656982 & 0.93366184 & 0.0067097493 \\
\hline & 8.5050951651 & 1.3728279579 & -1.4939695313 \\
\hline & & & \\
\hline C & 2.1561803061 & -3.3283611812 & 2.2944322381 \\
\hline & 2.6803540622 & -2.1544474205 & 1.7307507635 \\
\hline & 3.5445558512 & -1.372530183 & 2.490569159 \\
\hline & 3.8879211565 & -1.7386859743 & 907051851 \\
\hline & 3.3667693331 & -2.9037431163 & 4.3400158308 \\
\hline & 2.4998322457 & -3.6980293796 & 3.5879592594 \\
\hline  & 2.2499537673 & -1.6926467747 & 0.0096726184 \\
\hline $\mathrm{Ru}$ & 3.2232294044 & 0.2976960357 & -0.8771228295 \\
\hline $\mathrm{Cl}$ & 1.5818255739 & 93779 & 697694 \\
\hline & 0.4371861923 & -1.9694348089 & 0.0158 \\
\hline & -0.2371209732 & -2.486686425 & -1.0932253157 \\
\hline c & -1.5952396021 & -2.7614321956 & -1.0199878318 \\
\hline & -2.3112676074 & -2.5146671066 & 0.1496475493 \\
\hline & -1.6513226229 & -1.966934421 & 3042 \\
\hline & -0.28989 & $-1.69^{\circ}$ & 1.18 \\
\hline & -3.76043 & 23402 & 191 \\
\hline 0 & -4.1803322803 & -3.6970556717 & -0.6996262994 \\
\hline & -4.5576938795 & -2.4175654822 & 1.1288984021 \\
\hline & $-5.973^{\prime}$ & 1151 & 357 \\
\hline & -6.5 & 8601 & 77541 \\
\hline b & -6.05 & -1.0 & -0.7 \\
\hline & -7.7509993189 & -2.5170274986 & -0.5044155898 \\
\hline C & -8.4310404593 & -1.8944212343 & -1.5962468149 \\
\hline $\mathrm{C}$ & 2.9812704869 & -3.0738510524 & -0.972 \\
\hline C & 3.8 & -3. & -0 . \\
\hline r & 4.490 & -4.964 & 20885 \\
\hline $\mathrm{C}$ & 4.26839700 & -5.0244109645 & -2.5309761248 \\
\hline $\mathrm{C}$ & 3.4105134791 & -4.1020762339 & -3.1275682972 \\
\hline $\mathrm{C}$ & 2.7823605761 & -3.1281800965 & -2.3605940434 \\
\hline P & 1.7 & 1.82 & 0.1 \\
\hline $\mathrm{C}$ & 2.45 & 2.4 & 1.7 \\
\hline $\mathrm{C}$ & 2.415 & 1.58 & 2.86 \\
\hline $\mathrm{C}$ & 3.0185295725 & 1.9617613073 & 4.062181034 \\
\hline $\mathrm{C}$ & 3.6765123573 & 3.1864857958 & 4.1530373046 \\
\hline $\mathrm{C}$ & 3.727 & 4.023342897 & 3.0421864142 \\
\hline $\mathrm{C}$ & 3.1 & 3.6 & 1.8 \\
\hline $\mathrm{C}$ & -0.0 & 51542 & 3218 \\
\hline $\mathrm{C}$ & -0.8732016256 & 1.1166204078 & -0.5082883849 \\
\hline $\mathrm{C}$ & -2.2418355033 & 0.9935113969 & -0.3081003707 \\
\hline C & -2.7814316761 & 1.1808539338 & 0.9641530848 \\
\hline$C$ & -1.942 & 7239 & 2 . \\
\hline$C$ & -0.5 & 1.6 & 1.8 \\
\hline $\mathrm{C}$ & -4.2 & 332001 & 8941186 \\
\hline 0 & -4.6368940797 & 0.3619287856 & 2.2025704124 \\
\hline $\mathrm{N}$ & -5.0657846693 & 1.7120568416 & 0.4267110697 \\
\hline $\mathrm{C}$ & -6.5075142023 & 1.619836304 & 0.5139358823 \\
\hline 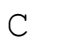 & -7.0422227194 & 1.9153646447 & $-0 . \varepsilon$ \\
\hline 0 & -6.3769848608 & 2.3856196489 & -1.7706758906 \\
\hline 0 & -8.3418067339 & 1.6369730497 & -0.9722668405 \\
\hline $\mathrm{C}$ & -8.9412107106 & 1.9325627171 & -2.2394106046 \\
\hline $\mathrm{C}$ & 1.4648 & 3340653 & -0.79380872 \\
\hline $\mathrm{C}$ & 0.8110882002 & 4.4480471482 & -0.1673788543 \\
\hline
\end{tabular}




\begin{tabular}{|c|c|c|c|}
\hline 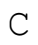 & 0.548226197 & 5.6178578113 & -0.8668635031 \\
\hline & 0.9151377097 & 5.7276096217 & -2.207892792 \\
\hline & 1.5308793174 & 4.6575500379 & -2.8452512296 \\
\hline & 1.8016203571 & 3.4841945993 & -2.142847194 \\
\hline & 5.2387038976 & 0.833869153 & 0.1643234135 \\
\hline & 5.2621234553 & -0.4887317494 & 929011 \\
\hline & 5.0653802379 & -0.7779233648 & 905 \\
\hline & 4.7645832473 & 0.2499529568 & -2.6194185232 \\
\hline & 4.6327278822 & 1.5783368665 & -2.1150039915 \\
\hline & 4.8558857681 & 1.8549621914 & -0.7533695617 \\
\hline & 5.7206440314 & 1.1760841801 & 1.5455006037 \\
\hline & 4.5602708895 & -0.0419462896 & -4.0738673046 \\
\hline & -7.0915458706 & 2.5762564098 & 1.5591817735 \\
\hline & -6.7734279962 & 0.5931265291 & 0.7684446481 \\
\hline & -6.1123940001 & -3.7629073384 & 0.8880782862 \\
\hline & -6.6989896962 & -2.2385330046 & 2.3059981879 \\
\hline & -4.3064254619 & -1.5 & 1.60 \\
\hline & -4.7045337842 & 2.2014008224 & -0.3823191175 \\
\hline & -2.887040946 & 0.7207154009 & -1.1382109934 \\
\hline & -0.4622134154 & 0.9519566049 & -1.4968379176 \\
\hline & 0.037232735 & 1.9473357722 & 2.6655306839 \\
\hline & -2.3679403525 & 1.6127056853 & 3.02184 \\
\hline & -2.1198231094 & -3.1992940662 & 305324 \\
\hline & 0.2869640278 & -2.7031068823 & -2.0138621488 \\
\hline & 0.2037193891 & -1.2907705849 & 2.0573988941 \\
\hline & -2.1831949315 & -1.7660309492 & 2.1742253154 \\
\hline & 7690575 & -3.9771734317 & 0.682 \\
\hline & 994 & -5.6718992465 & -0.684 \\
\hline & 4.7621199836 & -5.7806300378 & -3.1321611902 \\
\hline & 3.2336644024 & -4.1349033358 & -4.1979295695 \\
\hline & 2.1726692477 & -2.378982379 & -2.8532525311 \\
\hline & 0.5027657524 & 4.3671188403 & 0.870 \\
\hline & 4943 & 6.4 & -0.3 \\
\hline & 0.7048256049 & 6.6408831626 & -2.75 \\
\hline & 1.7877636091 & 4.7249277612 & -3.8973752142 \\
\hline & 2.2236900969 & 2.6326597507 & -2.6600329269 \\
\hline & -7.8350391817 & -1.9693800856 & -2.5082784637 \\
\hline & -8.6175901679 & -0.8415010029 & -1.3721035085 \\
\hline & -9.3692705833 & -2.4351979308 & 37958 \\
\hline & -9.9970503259 & 1.6931627592 & -2.1266123456 \\
\hline & -8.4868655354 & 1.3228002489 & -3.0232733451 \\
\hline & -8.8094436289 & 2.9879440629 & -2.4846967956 \\
\hline & 5.48213 & -1.3058162895 & 0.346 \\
\hline & 5.1118073703 & -1.8040098808 & 97228 \\
\hline & 4.3571681015 & 2.3804018982 & -2.787727385 \\
\hline & 4.7449832856 & 2.8726654227 & -0.3919293735 \\
\hline & 5.5081905388 & 0.1117167001 & -4.6005676774 \\
\hline & 3.8064844634 & 0.6171533512 & -4.5062497604 \\
\hline & 4.242841891 & -1.0753247461 & -4.2226742851 \\
\hline & 6.6655894912 & 1.7211377656 & 1.4549109263 \\
\hline & 5.0202510028 & 1.8129365803 & 2.0857421407 \\
\hline & 5.9173829816 & 0.278405372 & 2.133759459 \\
\hline & 1.913990586 & 0.6212626888 & 2.8150812868 \\
\hline & 2.9726827445 & 1.2973835108 & 4.9191240988 \\
\hline & 77504 & 3.4865426546 & 5.0839133374 \\
\hline & 4.2362607999 & 4.9794387898 & 3.1033019731 \\
\hline & 3.1590696649 & 4.3173840997 & 0.9974699746 \\
\hline
\end{tabular}




$\begin{array}{llcc}\mathrm{H} & 4.554068735 & -1.1071841273 & 4.3705558326 \\ \mathrm{H} & 3.6273816633 & -3.1940997822 & 5.3525090398 \\ \mathrm{H} & 2.0876700038 & -4.6068062323 & 4.0134931046 \\ \mathrm{H} & 1.4787498188 & -3.9524151423 & 1.7199144266 \\ \mathrm{H} & 3.9256467599 & -0.4518819971 & 2.0730471086 \\ \mathrm{H} & -7.769605948 & -2.4301274356 & 2.2134284439 \\ \mathrm{H} & -6.3162205611 & -2.7942083571 & 3.1649339692 \\ \mathrm{H} & -6.533461038 & -1.1726745905 & 2.4864881112 \\ \mathrm{H} & -8.1764579184 & 2.4638419302 & 1.6117550504 \\ \mathrm{H} & -6.6644873214 & 2.3354016021 & 2.5346489606 \\ \mathrm{H} & -6.8528705681 & 3.6151280816 & 1.3149688047\end{array}$

